\title{
Solvatationsdynamik an biologischen Grenzschichten
}

\author{
Dissertation \\ zur Erlangung des Doktorgrades \\ der Mathematisch-Naturwissenschaftlichen Fakultäten \\ der Georg-August-Universität zu Göttingen
}

vorgelegt von

Marco Thomas Seidel

aus Kassel

Göttingen 2003 
D7

Referent: Prof. Dr. J. Troe

Korreferent: Prof. Dr. B. Abel

Tag der mündlichen Prüfung: 05. Nov. 2003 


\section{Danksagung}

Ganz herzlich möchte ich Prof. Peter Vöhringer für die angenehme Betreuung während meiner Zeit am Max-Planck-Institut für biophysikalische Chemie in der Arbeitsgruppe „Biomolekulare und Chemische Dynamik“ danken. Mit seiner Diskussionsbereitschaft und seinem Erfahrungsschatz stand er mir stets mit Rat und Tat zur Seite, wodurch die hier vorgestellten Ergebnisse überhaupt erst möglich gemacht wurden. Sehr zum Gelingen dieser Arbeit trugen auch die exzellenten Arbeitsbedingungen in seiner Abteilung bei.

Ein besonderer Dank gilt Prof. Jürgen Troe für die Übernahme der Doktorvaterschaft und der Betreuung von Seiten der Universität. Ebenso möchte ich mich für die Übernahme des Korreferates bei Prof. Bernd Abel bedanken.

Bei allen Mitgliedern der gesamten Arbeitsgruppe 072 möchte ich mich für ihre vielfältige Hilfe bei der tagtäglichen Arbeit und der netten Zeit bedanken. Jeden einzelnen aufzuführen sprengt zwar fast den Rahmen dieser Danksagung, ich wage es aber dennoch: Nicole Breidenassel, Helge Bürsing, Eveline Heinemann, Jaydev Jethwa, Santi Kundu, Jörg Lindner, Donald Ouw, Raul Quiroz, Carlos Rudamas und Kathrin Winkler. Besondere Erwähnung verdient Helge, mit dem ich in den ersten eineinviertel Jahren Labortisch, Büro und damit auch sehr viel Zeit teilte. Ein herzlicher Dank gebührt weiterhin Evi, der „Guten Seele“ der Abteilung, dem „wandelnden Elektrowarenfachgeschäft" namens Jay und natürlich Kathrin, die sich in den letzten Jahren nicht nur meine wissenschaftlichen Problemchen anhören mußte.

In diesem Zusammenhang möchte ich auch die Jungs und Mädels von der Uni erwähnen, mit denen ich bevorzugt dann den wissenschaftlichen Austausch suchte, wenn ich nicht sonderlich erpicht darauf war, den Faßberg empor zu radeln (was seltsamerweise meist bei Regen der Fall war). Hervorzuheben ist dabei meine Ex-Heimat, namentlich Christian Grimm, Matthias Kling, Tobias Steinel und Jochen Zerbs (obwohl der ja erst später dazu kam), die mir in solchen Stunden bereitwillig Asyl und Kaffee gewährten, bis ich (oder der Bus) die nötige Energie aufbringen konnte. 
Für den Kampf an der Korrekturfront, die sich von Nordamerika über England bis ins Herzen Deutschlands erstreckte, haben sich Christoph Kappel, Matthias Kling, Peter Kutne, Jörg Lindner, Hendrik Nahler, Kathrin Winkler und Jochen Zerbs verdient gemacht. Ich denke, die meisten Felertäufel konnten erfolgreich in die Flucht geschlagen werden. Dem $\mathrm{AT}_{\mathrm{EX}} \mathrm{X}$-Lehrmeister Jörg Hahn und seinem Gesellen Christian Grimm sei natürlich auch für das „Schönmachen“ der Arbeit gedankt.

Bei Heiko Seeger und der Arbeitsgruppe von Prof. Thomas Heimburg möchte ich mich für die Durchführung der kalorimetrischen Messungen bedanken. Ebenso gilt mein Dank Gerhard Busse für die Einführung am Fluoreszenzspektrometer.

Stellvertretend für alle Mitarbeiter der Werkstätten des MPI's möchte ich Manfred Schmidt und Bernd Wallmann für ihre ausgezeichnete und zügige Arbeit danken. Besondere Erwähnung verdient die optische Werkstatt unter der Leitung von Wolfgang Sauermann, die eine wertvolle Hilfe in allen optischen Fragestellungen war. Ewig in Erinnerung wird mir der Ratschlag bleiben, daß ein dreckiger Spiegel immer noch besser ist, als ein schlecht geputzter. Das soll aber nicht heißen, daß ich nie geputzt hätte...!

Entscheidend am Fortgang der vorliegenden Arbeit war auch die „Kantine am MPI" beteiligt. Sie hat mich stets auf den Boden der kulinarischen Tatsachen geholt und mir so bewußt gemacht, daß es wichtigere Dinge als physikalische Chemie im Leben gibt...

...womit ich schlußendlich zu meinen Freunden und meiner Familie komme, denen ich ganz herzlich für die Unterstützung während meiner Studienzeit, der Promotion und darüberhinaus danken möchte. 


\section{Inhaltsverzeichnis}

$\begin{array}{lr}\text { Zusammenfassung } & 1\end{array}$

1 Einleitung 3

$\begin{array}{llr}2 & \text { Solvatationsdynamik } & 7\end{array}$

2.1 Prinzip der Solvatation . . . . . . . . . . . . . . . 7

2.2 Zeitabhängiger Fluoreszenz Stokes-Shift . . . . . . . . . . . . . . 10

2.3 Photon-Echo-Spektroskopie . . . . . . . . . . . . . . . . . . 12

2.3.1 Vier-Wellen-Mischprozesse . . . . . . . . . . . . 13

2.3.2 3-Puls Photon-Echo Peakshift . . . . . . . . . . . 17

2.3.3 Transient-Grating-Spektroskopie . . . . . . . . . . 19

3 Biologische Modellsysteme 21

3.1 Wasser .............................. 22

3.2 Mizellen . . . . . . . . . . . . . . . . . 25

3.2.1 Struktur . . . . . . . . . . . . . . 25

3.2 .2 Solvatationsdynamik . . . . . . . . . . . . 25

3.3 Inverse Mizellen . . . . . . . . . . . . . . . . . . . . . . . . 28

3.3.1 Struktur .......................... 28

3.3.2 Solvatationsdynamik . . . . . . . . . . . . . . 31

3.3.3 MD-Simulationen . . . . . . . . . . . . . . . . 34

3.4 Lipid-Vesikel . . . . . . . . . . . . . . . . . . . . . . . . . . . 35 
3.4.1 Phospholipide . . . . . . . . . . . . . . . 35

3.4 .2 Vesikel . . . . . . . . . . . . . . 36

3.4.3 Polymorphismus . . . . . . . . . . . 37

3.4.4 Solvatationsdynamik . . . . . . . . . . . . . . 39

3.4.5 MD-Simulationen . . . . . . . . . . . . . . 42

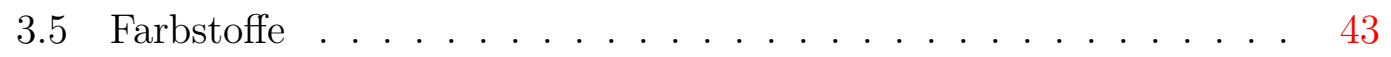

3.5.1 Laurdan . . . . . . . . . . . . . . . . . 43

3.5 .2 HIDCI ........................ 46

4 Experimentelle Techniken $\quad 47$

4.1 Stationäre Meßmethoden . . . . . . . . . . . . . . . . . . . . 47

4.1.1 Absorptionsspektroskopie . . . . . . . . . . 47

4.1.2 Emissions- und Anregungsspektroskopie . . . . . . . . . . 48

4.1.3 Dynamische Differentialkalorimetrie . . . . . . . . . . . . 48

4.2 Zeitkorreliertes Einzelphotonenzählen . . . . . . . . . . . . . 48

4.2 .1 Experimenteller Aufbau . . . . . . . . . . . . 49

4.2.2 Charakterisierung des Meßsystems . . . . . . . . 51

4.2 .3 Meßprozedur . . . . . . . . . . . . . . 52

4.3 Laser für Ultrakurzzeit-Untersuchungen . . . . . . . . . . . . . 53

4.4 Fluoreszenzkonversionsspektroskopie . . . . . . . . . . . 56

4.4.1 Prinzip der Fluoreszenzkonversionsspektroskopie . . . . . . 56

4.4 .2 Experimenteller Aufbau .............. 61

4.4.3 Charakterisierung des Meßsystems ......... 63

4.4 .4 Meßprozedur ..................... 64

4.5 Photon-Echo-Spektroskopie . . . . . . . . . . . 66

4.5.1 Nichtkollinearer Optisch-Parametrischer Verstärker . . . 67

4.5.2 Prinzip der Photon-Echo-Spektroskopie . . . . . . . . 70

4.5.3 Experimenteller Aufbau . . . . . . . . . . . . 71

4.5 .4 Meßprozedur . . . . . . . . . . . . 73 
4.6 Verwendete Chemikalien und Probenpräparation . . . . . . . . . 76

4.6.1 Präparation der Lipid-Vesikel . . . . . . . . . . . 77

4.6.2 Präparation der Inversen Mizellen . . . . . . . . . . . . 78

$\begin{array}{lll}5 & \text { Lipid-Vesikel } & 79\end{array}$

5.1 Untersuchungen an Laurdan . . . . . . . . . . . . . . 80

5.2 Untersuchungen an Lipid-Vesikeln . . . . . . . . . . . . . 83

5.2.1 Kalorimetrische Ergebnisse . . . . . . . . . . . . . 84

5.2.2 Spektroskopische Eigenschaften der reinen Membran . . . 86

5.2.3 Spektroskopische Eigenschaften der markierten Membran . 87

5.3 Anisotropieeigenschaften . . . . . . . . . . . . . . . . 92

5.4 Solvatationsdynamik . . . . . . . . . . . . . . . . 97

5.4.1 Zeitaufgelöste Fluoreszenzspektren . . . . . . . . . . . 97

5.4.2 Solvatation an der Lipidmembran/Wasser-Grenzschicht . . 107

5.4.3 Ultraschnelle Solvatation . . . . . . . . . . . . . . . 117

5.5 Diskussion der Solvatationsdynamik . . . . . . . . . . . . . . . . 121

5.6 Resümee - Lipid-Vesikel . . . . . . . . . . . . . . . . . . . . . 129

6 Inverse Mizellen 131

6.1 Stationäre Eigenschaften inverser Mizellen . . . . . . . . . . . . . 131

6.1.1 Charakterisierung inverser Mizellen . . . . . . . . . . 131

6.1.2 Stationäre spektroskopische Eigenschaften . . . . . . . . . 134

6.2 Solvatationsdynamik in Wasser . . . . . . . . . . . . 136

6.3 Solvatationsdynamik in inversen Mizellen . . . . . . . . . . . . . . 142

6.4 Schwingungsdynamik . . . . . . . . . . . . . . . . . . . 149

6.5 Resümee - Inverse Mizellen . . . . . . . . . . . . . . . . 153

$\begin{array}{llr}7 & \text { Ausblick } & 155\end{array}$

$\begin{array}{lr}\text { Literaturverzeichnis } & 159\end{array}$ 
viii

INHALTSVERZEICHNIS

Abbildungsverzeichnis

175

Tabellenverzeichnis

179

Lebenslauf

181 


\section{Zusammenfassung}

Die vorliegende Arbeit befaßt sich mit der ultraschnellen Solvatationsdynamik an Grenzschichten ausgesuchter biologischer Modellsysteme.

Als ein Modell für biologische Membranen dient eine synthetische Phospholipidmembran/Wasser-Grenzschicht in unilamellaren Vesikeln. Zur Bestimmung der Solvatationsdynamik an solchen Grenzschichten wird der zeitabhängige Fluoreszenz Stokes-Shift der Farbstoffsonde Laurdan nach instantaner elektronischer Anregung herangezogen. Dieser Farbstoff ist nicht-kovalent in der Membran verankert und in der Region der Lipid-Kopfgruppen lokalisiert.

Mit der Methode des zeitkorrelierten Einzelphotonenzählens (TCSPC) wurden die in dieser Membranumgebung stattfindenden strukturellen Relaxationen auf einer Nanosekunden-Zeitskala charakterisiert, wobei insbesondere der in Lipidmembranen auftretende Polymorphismus mit Hilfe temperaturabhängiger Studien untersucht wurde. Dabei zeigte sich, daß die Solvatation in heterogenen Umgebungen im Vergleich zu reinen Flüssigkeiten signifikant verlangsamt ist. Insbesondere der Hauptphasenübergang, der mit einem Schmelzen der hydrophoben Kettenregion der Membranen einhergeht, hat dabei einen erheblichen Einfluß auf die beobachteten Dynamiken. Durch einen Vergleich mit zeitaufgelösten Anisotropiemessungen konnte gezeigt werden, daß die Solvatation auf einer Nanosekunden-Zeitskala vorrangig durch diffusive Bewegungen des Chromophors in der eingeschränkten Lipidmembranumgebung bestimmt ist.

Die TCSPC-Technik erlaubt aufgrund ihrer limitierten Zeitauflösung, nur die langsameren Komponenten der Solvatationsdynamik an der Grenzschicht zu erfassen. Im Rahmen dieser Arbeit wurde daher ein Fluoreszenzkonversionsexperiment aufgebaut, welches Untersuchungen bis in den sub-Pikosekundenbereich ( $\sim 500 \mathrm{fs}$ ) ermöglicht und erstmals zur Bestimmung der ultraschnellen Solvatation in vesikulären Umgebungen herangezogen wurde. Es zeigte sich, daß auf diesen Zeitskalen ultraschnelle Komponenten eine Rolle spielen, die vermutlich der Solvatation durch Wassermoleküle im Bereich der Lipidkopfgruppen zuzuordnen sind. Dabei ist die Dynamik im Vergleich zu reinem Wasser jedoch um etwa einen Faktor 3 verlangsamt. Es wurde weiterhin festgestellt, daß ein Großteil der Dyna- 
miken noch innerhalb der Zeitauflösung des Fluoreszenzkonversionsexperimentes stattfindet und sich damit der Beobachtung durch die hier angewendeten Techniken entzieht.

Als zweites Modellsystem werden in dieser Arbeit inverse Mizellen betrachtet, welche sich hervorragend zum systematischen Studium eingeschränkter Wasserumgebungen eignen, da sich der Grad der Einschränkung über die Größe des in diesen inversen Mizellen eingeschlossenen Wassernanotröpfchens variieren läßt. Die Solvatationsdynamik wurde dabei mittels eines selektiv im Wassereinschluß der inversen Mizellen gelösten Indocarbocyanin-Farbstoffes bestimmt.

Als experimentelle Technik kam in diesen Systemen erstmals die Photon-EchoSpektroskopie zum Einsatz, die die Bestimmung ultraschneller Zeitkomponenten auf einer Femtosekunden-Zeitskala ermöglicht. Um die im Wassernanotröpfchen auftretenden Dynamiken einordnen zu können, wurden komplementäre Untersuchungen desselben Chromophors in der reinen Wasserphase durchgeführt. Die Solvatationsdynamik in reinem Wasser weist im Wesentlichen zwei charakteristische Zeitskalen auf. Die Komponente um 1 ps ist übergedämpften, kollektiven Translationsmoden des reinen Wassers zuzuschreiben, während die Komponente um 10 ps aus der Solvatation durch diffusive Reorientierungsdynamik der Wassermoleküle resultiert.

Das Einbringen des Chromophors in das Wassernanotröpfchen inverser Mizellen verursacht keine ausgeprägte Veränderung der Solvatationsdynamik, d. h. die für reines Wasser charakteristischen strukturellen Relaxationsmoden bestimmen auch weiterhin die Solvatation innerhalb des Wassereinschlusses inverser Mizellen. Die Erhöhung des Einschränkungsgrades durch eine Verringerung der Mizellengröße zeigt dabei einen überraschend geringen Effekt auf die zugrundeliegenden Dynamiken der Solvatation. Dieses experimentelle Ergebnis steht jedoch im Einklang mit Vorhersagen aus neueren molekulardynamischen Simulationen von Faeder und Ladanyi.

Die Ergebnisse der durchgeführten Studien lassen darauf schließen, daß in heterogenen, eingeschränkten Umgebungen langsame strukturelle Relaxationsmechanismen auf einer Nanosekunden-Zeitskala eine Rolle spielen, die in reinem Wasser nicht beobachtet werden. An einem Modellsystem für biologische Membranen konnte gezeigt werden, daß für diese Komponenten die eingeschränkte Eigendynamik des Chromophors verantwortlich ist. Darüberhinaus wird die Solvatation durch ultraschnelle Zeitkomponenten auf einer Pikosekunden-Zeitskala dominiert, welche aus der Dynamik des Wassers resultiert. Während diese im Falle der inversen Mizellen für das reine Lösungsmittel charakteristisch sind, erscheinen sie in Lipidumgebungen deutlich verlangsamt, was auf eine stark eingeschränkte Wasserumgebung in der Membran schließen läßt. 


\section{Kapitel 1}

\section{Einleitung}

Chemische Reaktionen in kondensierter Phase sind aufgrund ihrer unbestreitbaren Relevanz in Natur und Technik von besonderem Interesse. Da eine größtmögliche Kontrolle über chemische Reaktionen eines der grundlegenden Ziele der Naturwissenschaften darstellt, ist es unabdingbar, die in Flüssigkeiten ablaufenden Prozesse im Detail zu verstehen. Weil sich die relevanten Zeitskalen der Dynamik in flüssiger Phase allerdings bis hin zu wenigen Femtosekunden erstrecken, waren es zunächst experimentelle Beschränkungen, die das eingehende Studium der dort stattfindenden Vorgänge limitierten. Mit der Entwicklung ultraschneller, gepulster Laser war aber schließlich die Möglichkeit gegeben, auch die schnellsten Elementarprozesse in Flüssigkeiten zu untersuchen. Seitdem hat das Studium der Dynamik chemischer Prozesse eine wissenschaftliche Revolution erfahren, was nicht zuletzt mit der Vergabe des Nobelpreises 1999 auf dem Gebiet der Femtosekundenspektroskopie zum Ausdruck kommt [1]. Heutzutage existiert durch den rasanten experimentellen Fortschritt in Verbindung mit einer Vielzahl theoretischer Modellvorstellungen ein weitreichendes Verständnis der in reinen Flüssigkeiten stattfindenden Vorgänge [2,3].

Der Wissensstand bezüglich der ultraschnellen Dynamiken ändert sich jedoch dramatisch beim Übergang von reinen Flüssigkeiten auf heterogene bzw. mikroheterogene Systeme, wie sie insbesondere in der Biologie angetroffen werden. Als Beispiel seien hier die in biologischen Organismen vorliegenden Membranen oder auch die als Enzyme fungierenden Proteine genannt. Aber auch in der klassischen Chemie sind solche Systeme von Interesse, beispielsweise in der heterogenen Katalyse. Wie unterschiedlich die genannten Strukturen auch sein mögen, an den Grenzschichten solcher Systeme wird stets eine Veränderung der unmittelbaren Lösungsmittelumgebung beobachtet. Viele biochemische Prozesse in der Natur finden in solch eingeschränkten wäßrigen Umgebungen statt, wodurch sich im Gegensatz zur reinen Wasserphase stark unterschiedliche physikochemische Bedingungen z. B. bezüglich Polarität, Viskosität oder pH-Wert ergeben. Es wird 
vermutet, daß bestimmte Prozesse in der Natur überhaupt erst durch solche Modifikationen der Umgebung ablaufen können. Beispielsweise wird an Grenzschichten zwischen Wasser und Lipidmembranen das starre Wasserstoffbrückennetzwerk des reinen Wassers signifikant gestört, was den Transport von Ionen und Molekülen durch die Membran über sogenannte Ionenkanäle vermutlich erst möglich macht. Das Wasser in diesen eingeschränkten Systemen weist infolgedessen eine stark modifizierte Dynamik im Vergleich zur reinen Wasserphase auf.

Dabei steht das Studium der ultraschnellen Dynamiken in heterogenen Systemen im Gegensatz zu den reinen Flüssigkeiten erst am Anfang. Trotz der offensichtlichen Relevanz dieser Umgebungen bietet sich hier ein bislang nur wenig betretenes Forschungsfeld, das einen interessanten Einblick in die ablaufenden Prozesse auf molekularer Ebene ermöglicht. Ziel der vorliegenden Dissertation ist es daher, wäßrige Grenzschichten an ausgewählten biologischen Modellsystemen auf ihre ultraschnelle Solvatationsdynamik hin zu untersuchen, um grundlegende Erkenntnisse über diese elementaren Vorgänge in der Natur zu gewinnen.

\section{Wahl der Methoden und Modellsysteme}

Membranen stellen aufgrund ihrer biologischen Relevanz eines der wichtigsten Strukturelemente in der Biologie dar. Sie dienen nicht nur als Abgrenzung von Zellen und Zellorganellen, sondern stellen darüberhinaus auch eine Matrix für eine Vielzahl von Proteinen dar, die entscheidend für das Funktionieren biologischer Systeme verantwortlich sind $[4,5,6]$.

Als einfaches Modellsystem für biologische Membranen werden daher in der vorliegenden Arbeit synthetische Lipidmembran/Wasser-Grenzschichten in unilamellaren Vesikeln gewählt. Die Vesikel bestehen aus definierten Doppelschichten eines Phosphatidylphosphocholins (DMPC) und eignen sich hervorragend zum systematischen Studium der Solvatationsdynamik an biologischen Grenzschichten. Die Membran wird mit dem Fluoreszenzfarbstoff Laurdan markiert, der aufgrund seiner langen aliphatischen Kette nicht-kovalent in der Membran verankert und dessen Chromophor selektiv an der Grenzschicht lokalisiert ist. Solche Fluoreszenzmarker eignen sich daher als Sonde für die Strukturrelaxations- bzw. Solvatationsdynamik an biologischen Grenzschichten. Zur Untersuchung der zugrundeliegenden Dynamiken wird vorrangig der zeitabhängige Stokes-Shift der durch einen ultrakurzen Laserpuls initiierten Fluoreszenz herangezogen. Desweiteren werden die Anisotropieeigenschaften an der Grenzschicht eingehend untersucht. Dazu werden eine Reihe von frequenzaufgelösten (stationäre Absorptionsund Emissionsspektroskopie) und zeitaufgelösten fluoreszenzspektroskopischen Methoden (Zeitkorreliertes Einzelphotonenzählen und Fluoreszenzkonversionsspektroskopie) durchgeführt. Mit Hilfe dieser Techniken ist es möglich, die Dynamik der Solvatation über ein extrem weites Zeitfenster vom Nanosekundenbereich 
bis in den sub-Pikosekundenbereich zu charakterisieren. Darüberhinaus werden temperaturabhängige Studien der Dynamiken an der Lipidmembran/WasserGrenzschicht durchgeführt, wobei deutliche Veränderungen der Solvatationsdynamik beim Übergang zwischen verschiedenen thermodynamischen Phasen der Membran (Polymorphismus) beobachtet werden.

Als ein zweites Modellsystem zum Studium ultraschneller Prozesse in eingeschränkten Umgebungen werden sogenannte inverse Mizellen gewählt [7,8]. Es handelt sich dabei um Wassernanotröpfchen, die in einer organischen Phase mit Hilfe von Detergentien emulgiert sind. Das eingeschlossene Wasser der inversen Mizellen unterscheidet sich erheblich von reinem Wasser und eignet sich als ideales Modellsystem eingeschränkter Wasserumgebungen, da sich der Grad der Einschränkung einfach über die Größe des untersuchten Wassereinschlusses variieren läßt. Solche Strukturen werden in der Natur beispielsweise bei der Fusion von Membranen angetroffen, sie haben aber auch außerordentliche Bedeutung in der chemischen Katalyse oder der Synthese neuartiger Materialien. Das Innere inverser Mizellen wird daher auch als „Mikroreaktor" bezeichnet. Darüberhinaus werden sie als vielversprechende "Transportsysteme“ von Pharmazeutika gehandelt. Die praktischen Einsatzmöglichkeiten sind extrem vielfältig [7,8,9].

In der vorliegenden Arbeit wird repräsentativ das ternäre System Isooktan/ AOT/Wasser untersucht. Als Sonde für die ultraschnelle Solvatation kommt der Farbstoff HIDCI zum Einsatz, der sich selektiv in der Wasserphase der inversen Mizellen löst. Um den Einfluß des Einschränkungsgrades besser zu verstehen, werden unterschiedlich große Wassereinschlüsse betrachtet. Die Solvatationsdynamik in den Wassernanotröpfchen inverser Mizellen wird hier erstmals mit Methoden der Photon-Echo-Spektroskopie untersucht. Das durchgeführte 3-Puls PhotonEcho Peakshift Experiment erlaubt es, auch die ultraschnellen Zeitkomponenten der Wasserdynamik aufzulösen. Um die beobachteten Prozesse in den inversen Mizellen einordnen zu können, werden zum Vergleich analoge Messungen an reinem Wasser durchgeführt. Ergänzend zu den Untersuchungen zur Solvatationsdynamik werden Transient-Grating-Messungen durchgeführt, um die Schwingungsstruktur des Chromophors innerhalb des Wassernanotröpfchens zu studieren. 


\section{Gliederung}

Die vorliegende Arbeit gliedert sich wie folgt:

- In Kapitel 2 sollen die grundlegenden Prinzipien zur dynamischen Solvatation in Flüssigkeiten vorgestellt werden. Dabei wird insbesondere auf Methoden zur Bestimmung der Dynamiken auf ultrakurzen Zeitskalen eingegangen. Es handelt sich dabei um den zeitabhängigen Fluoreszenz StokesShift (TDFSS) und den 3-Puls Photon-Echo Peakshift (3PEPS).

- Kapitel 3 befaßt sich mit den in dieser Arbeit untersuchten biologischen Modellsystemen, d. h. insbesondere den inversen Mizellen und den LipidVesikeln. Dabei wird einerseits auf deren Struktur und andererseits auf frühere Untersuchungen zur Solvatationsdynamik an solchen Systemen eingegangen.

- Die experimentellen Techniken zur Durchführung der Untersuchungen zur Solvatationsdynamik an biologischen Grenzschichten werden in Kapitel 4 eingehend beschrieben. Die Schwerpunkte liegen dabei auf dem zeitkorrelierten Einzelphotonenzählexperiment, dem neu aufgebauten Fluoreszenzkonversionsexperiment und dem Photon-Echo-Experiment.

- Kapitel 5 widmet sich den Untersuchungen an Lipid-Vesikeln mit Betonung auf der Solvatation an Lipidmembran/Wasser-Grenzschichten. Dabei kamen verschiedene Methoden zur Bestimmung des zeitabhängigen StokesShifts zur Anwendung. Die experimentellen Ergebnisse und deren Auswertung werden ausführlich präsentiert, woran sich eine eingehende Diskussion der erhaltenen Erkenntnisse anschließt.

- Dem Modellsystem der inversen Mizellen wendet sich schließlich Kapitel 6 zu. Die ultraschnelle Solvatationsdynamik in den Wassernanotröpfchen der inversen Mizellen im Vergleich zur Dynamik in reinem Wasser wurde hier mit Hilfe des Photon-Echo-Experimentes untersucht. Wieder werden die Ergebnisse sowie deren Auswertung dargestellt, woran sich eine ausführliche Diskussion anschließt.

- Das abschließende Kapitel 7 gibt einen kurzen Ausblick auf zukünftige Studien zur Solvatationsdynamik an biologischen Grenzschichten. 


\section{Kapitel 2}

\section{Solvatationsdynamik}

In diesem Kapitel werden die grundlegenden Prinzipien zur dynamischen Solvatation in Flüssigkeiten (Kapitel 2.1) und Methoden zu deren Bestimmung vorgestellt. Auf den bei der Solvatation auftretenden dynamischen Stokes-Shift und der damit verbundenen Solvatationskorrelationsfunktion $S(t)$ wird in Kapitel 2.2 eingegangen. $S(t)$ stellt die zentrale Größe der Solvatationsdynamik im angeregten Zustand dar und wird in zeitaufgelösten Fluoreszenzmeßmethoden wie dem zeitkorrelierten Einzelphotonenzählen und der Fluoreszenzaufkonvertierung bestimmt.

Ein anderer Ansatz zum Studium der Solvatation ergibt sich aus der Beobachtung der Korrelationsfunktion $M(t)$ der Gleichgewichtsfluktuationen der elektronischen Energielücke, welche die zugrundeliegenden Dynamiken über Linienverbreiterungsmechanismen im betrachteten System liefert. Das 3-Puls PhotonEcho Peakshift Experiment (3PEPS) stellt die entsprechende Meßtechnik dar und wird in Kapitel 2.3 eingehend beschrieben.

Beide in diesem Kapitel beschriebenen Techniken wurden in der vorliegenden Arbeit zur Bestimmung der Solvatationsdynamik an ausgesuchten biologischen Grenzschichten genutzt.

\subsection{Prinzip der Solvatation}

Chemische Reaktionen zeichnen sich durch das Brechen und Bilden von chemischen Bindungen aus, was mit einer Veränderung der elektronischen Strukturen der reagierenden Spezies während der Reaktion einhergeht. Gegenüber Reaktionen in der Gasphase hat in kondensierter Phase das Lösungsmittel einen erheblichen Einfluß auf die veränderten elektronischen Strukturen im Verlauf der Reaktion. Um ein vollständiges Verständnis chemischer Reaktionen zu erhalten, ist es daher 
unumgänglich, den Einfluß des Lösungsmittels im Detail zu verstehen. Das Studium des Lösungsmittelbeitrags gestaltet sich in der Praxis allerdings als schwierig, da sich Beiträge der eigentlichen Reaktion und der reinen Solvatation durch das Lösungsmittel überlagern. Daher werden nicht-reaktive Spezies zum Studium der Solvatation herangezogen, wobei vorausgesetzt werden muß, daß die Änderung des Systems durch die eingebrachte Substanz klein bleibt und die zugrundeliegende Dynamik nur unwesentlich beeinflußt wird. Üblicherweise werden dazu organische Farbstoffmoleküle als sogenannte Farbstoffsonden verwendet.

Moleküle in flüssiger Phase befinden sich in ständiger Bewegung und weisen eine charakteristische zeitliche und räumliche Dynamik auf. In Abwesenheit äußerer Störungen befindet sich ein gelöster Farbstoff daher in einem definierten dynamischen Gleichgewichtszustand mit dem Lösungsmittel, welcher durch die Lösungsmittel-Lösungsmittel-Wechselwirkungen und zusätzlich durch die Lösungsmittel-Farbstoff-Wechselwirkungen bestimmt ist. Die Dynamik der Flüssigkeit in diesem Gleichgewichtszustand ist von grundlegendem Interesse und kann mittels der 3-Puls Photon-Echo Peakshift Spektroskopie bestimmt werden (siehe dazu Kapitel 2.3).

Das Studium der Solvatationsdynamik ist weiterhin über den angeregten Zustand des eingebrachten Farbstoffes möglich. Im Gegensatz zur Gleichgewichtsdynamik des Lösungsmittels im Grundzustand, beschreibt die Solvatation im angeregten Zustand die zeitabhängige Relaxation der Lösungsmittelmoleküle aufgrund einer instantanen Änderung des elektronischen Zustandes und ist daher auch in einen Zusammenhang mit den sich ändernden elektronischen Strukturen bei einer chemischen Reaktion zu bringen. Durch die instantane Anregung der Farbstoffsonde befinden sich die Lösungsmittelmoleküle, welche zuvor im Gleichgewicht mit der gelösten Spezies im Grundzustand vorlagen, in einem Nichtgleichgewichtszustand. Infolgedessen verändern sie daher ihre Position und/oder Orientierungen, um in ein erneutes Gleichgewicht mit dem neu erzeugten Zustand zu gelangen.

Die Änderung der elektronischen Dichteverteilung im angeregten Zustand in polaren Flüssigkeiten wird in der Mehrzahl der bisher veröffentlichten Arbeiten zur Solvatationsdynamik als das Schalten eines Dipolmomentes des verwendeten Farbstoffes bei elektronischer Anregung verstanden. Im Grundzustand besitzt der Farbstoff nur ein geringes bzw. kein Dipolmoment und befindet sich im Gleichgewicht mit seiner Umgebung. Bei elektronischer Anregung wird dieses in seiner Größe bzw. seiner Ausrichtung verändert, wodurch es zu einer entsprechenden Stabilisierung des veränderten Dipols durch das Lösungsmittel kommt (siehe Abbildung 2.1). Diese Art der Solvatation wird als polare bzw. dipolare Solvatation bezeichnet und ist sowohl experimentell als auch theoretisch eingehend untersucht worden $[3,10,11,12,13]$. Darüberhinaus gibt es aber auch noch weitere Modellvorstellungen, welche z. B. die Solvatation in unpolaren Medien beschreiben. Als Beispiel sei hier das viskoelastische Modell von Mark Berg angeführt, welches die Dynamik der Solvatation mit mechanischen Eigenschaften des Systems be- 


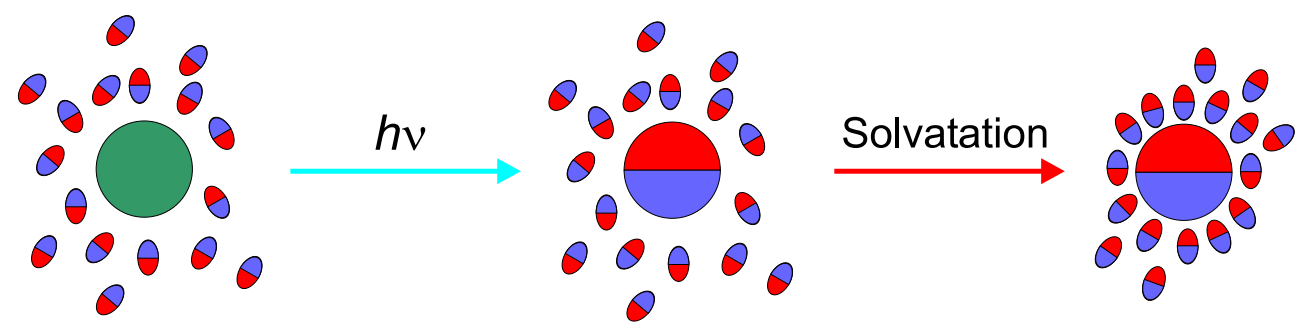

Abbildung 2.1: Erzeugung und Solvatation eines Dipoles in einem dipolaren Lösungsmittel in schematischer Darstellung.

schreibt $[14,15,16]$. Eine elektronische Anregung führt neben einer eventuellen Änderung des Dipolmomentes auch zu einer Änderung der Form und/oder Größe des Farbstoffmoleküls. Der auf diese Weise erzeugte Nichtgleichgewichtszustand gibt wiederum Anlaß zur Reorganisation der Lösungsmittelmoleküle. Während dieser Effekt in polaren Flüssigkeiten meist vernachlässigbar ist, kann er in unpolaren Flüssigkeiten bestimmend sein. Einen umfassenden Überblick über die Mechanismen und Theorien der Solvatation in polaren und unpolaren Umgebungen geben Bagchi und Biswas [3].

Beim Studium der Solvatationsdynamik sind eine Vielzahl unterschiedlicher Fragestellungen von Interesse. Insbesondere stellt sich die Frage, auf welchen Zeitskalen die Solvatation stattfindet und wie ihr zeitlicher Verlauf beschrieben werden kann. Weiterhin gilt es, die molekularen Mechanismen zu ergründen, die dem beobachteten Solvatationsverlauf zugrunde liegen und den Einfluß äußerer Parameter zu bestimmen.

Zur Klärung dieser Fragestellung sind eine Vielzahl unterschiedlichster Modelle propagiert worden. Die einfachsten Ansätze behandeln das Lösungsmittel als reines Dielektrikum, das durch die makroskopische Größe der Dielektrizitätskonstanten beschrieben wird. Molekulare Eigenschaften des Lösungsmittels werden bei diesen Beschreibungen außer Acht gelassen. Eine Behandlung dieser Theorien bietet beispielsweise Fröhlich [17]. Diese einfachen Kontinuumsmodelle sind jedoch nicht in der Lage die komplizierten Vorgänge der Solvatation in komplexeren Systemen, wie z. B. Wasser, ausreichend zu beschreiben. In solchen Fällen muß auf die molekularen Eigenheiten der Lösungsmittelmoleküle eingegangen werden. So werden für die möglichen Freiheitsgrade der an der Solvatation beteiligten Moleküle beispielsweise Rotationen, Librationen und Translationen herangezogen. Im mechanistischen Bild der Brown'schen Oszillatoren wird eine Flüssigkeit als ein System stark gekoppelter gedämpfter harmonischer Oszillatoren beschrieben, die durch ihren Ort und ihre Auslenkung charakterisiert sind. Dieses Modell hat sich bei der Beschreibung komplizierter Systeme als äußerst aussagekräftig erwiesen und wird von Mukamel ausführlich behandelt [18]. 


\subsection{Zeitabhängiger Fluoreszenz Stokes-Shift}

Die am weitesten angewandte und am leichtesten interpretierbare Technik zur Untersuchung der Solvatationsdynamik ist die Messung des zeitabhängigen Fluoreszenz Stokes-Shifts (TDFSS, Time-Dependent Fluorescence Stokes-Shift). Die TDFSS-Technik mißt eine zeitabhängige Verschiebung des Emissionsspektrums, die durch die Relaxation des Systems nach elektronischer Anregung hervorgerufen wird $[2,19,20,21,22,23,24,25,26]$.

Die zeitliche Entwicklung nach elektronischer Anregung eines Ensembles von Chromophoren läßt sich vereinfacht in einem Zwei-Niveau-System beschreiben. In Lösung muß zusätzlich zum systemeigenen Hamilton-Operator dieses Ensembles die Kopplung an das Lösungsmittelbad berücksichtigt werden. Diesem Umstand wird Rechnung getragen, indem die System-Bad-Wechselwirkungsenergien des Zwei-Niveau-Systems mit elektronischen Grundzustand $|\mathrm{g}\rangle$ und angeregtem Zustand $|e\rangle$ in Abhängigkeit einer verallgemeinerten Lösungsmittelkoordinate $q$ dargestellt werden (siehe Abbildung 2.2 links). Dabei umfaßt die verallgemeinerte Koordinate $q$ sämtliche molekularen Freiheitsgrade des Bades, die an die Übergangsdipolmomente der betrachteten Chromophore koppeln. Vor elektro-
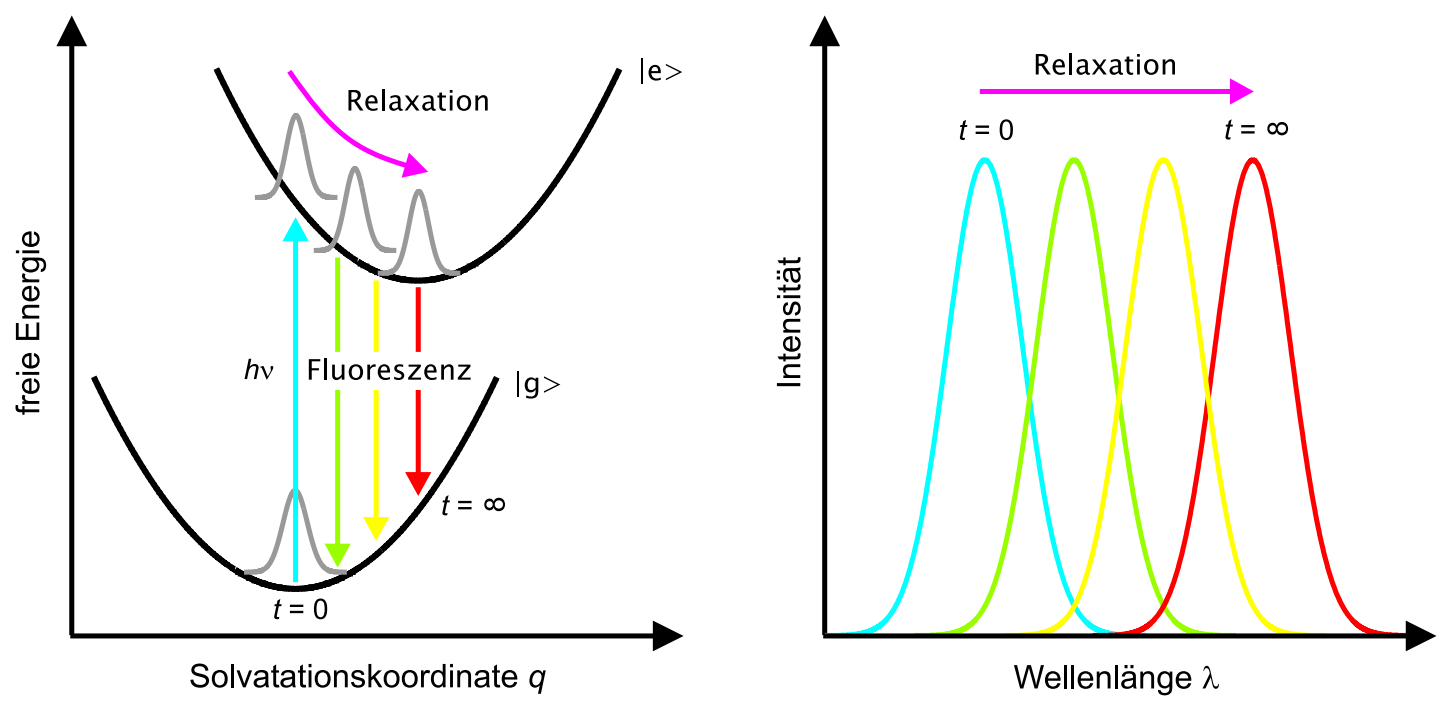

Abbildung 2.2: Prinzip der Beobachtung der Solvatationsdynamik über den dynamischen Stokes-Shift. Links: Anregung einer Population vom Grundzustand in den angeregten Zustand führt zur Solvatation auf den System-BadWechselwirkungspotentialen im angeregten Zustand, was direkt über die zeitabhängige Energieänderung der Fluoreszenzspektren beobachtet werden kann; Rechts: Zeitabhängige Fluoreszenzspektren, die die Rotverschiebung der Emission bis zur vollständigen Relaxation des Systems bei $t=\infty$ demonstrieren. 
nischer Anregung befindet sich das Ensemble im thermischen Gleichgewicht mit seiner Umgebung, was als Gaußverteilung auf der Parabel der freien SystemBad-Wechselwirkungsenergie im Grundzustand $|\mathrm{g}\rangle$ dargestellt ist (siehe Abbildung 2.2 links). Durch Anregung mit einem ultrakurzen Laserpuls der Energie $h \nu$ wird gemäß der Born-Oppenheimer-Näherung derjenige Teil dieser Verteilung, dessen Energien in Resonanz mit dem Spektrum des Laserpulses stehen, in den angeregten Zustand |e $\rangle$ überführt. Die neu erzeugte Verteilung wird durch eine Gaußfunktion auf der Kurve der freien Energie im angeregten Zustand dargestellt (siehe Abbildung 2.2 links). Sie repräsentiert einen Nichtgleichgewichtszustand im angeregten Zustand aufgrund der relativen Verschiebung der System-BadWechselwirkungspotentiale zueinander. Unter der Voraussetzung, daß das System während der elektronischen Anregung, d.h. im Zeitbereich der Pulsdauer, nicht relaxiert, wird das Emissionsspektrum der angeregten Spezies zu diesem Zeitpunkt identisch zum Spektrum des Anregungspulses sein und liegt bei $\Delta E(t=0)$, der Energielücke zwischen Grund- und angeregtem Zustand zum Zeitpunkt Null. Die nun stattfindende Reorganisierung des Lösungsmittels um das angeregte System führt zu einer Stabilisierung im angeregten Zustand, was mit einer Verschiebung und Verbreiterung der Verteilung einhergeht. Nach vollständiger Relaxation $(\Delta E(t=\infty))$ entspricht das Emissionsspektrum schließlich dem stationären Spektrum. Es kann lediglich eine weitere Abnahme der Fluoreszenzintensität aufgrund der endlichen Lebensdauer des angeregten Zustandes beobachtet werden. In stark gehinderten Systemen, wie sie Gegenstand der vorliegenden Arbeit sind, kann die Zeit zur vollständigen Solvatation des untersuchten Systems jedoch auch länger als die Fluoreszenzlebensdauer des angeregten Zustandes sein.

Die Verschiebung der Emissionsspektren im Laufe der Solvatation zu geringeren Energien, wie in Abbildung 2.2 rechts dargestellt, wird als dynamischer StokesShift bezeichnet und beträgt $\Delta E(t)-\Delta E(t=\infty)$. Die Solvatationskorrelationsfunktion $S(t)$, auch Stokes-Shift Funktion genannt, entspricht dem dynamischen Stokes-Shift bezogen auf den gesamten, d. h. stationären Stokes-Shift. Sie beschreibt die Dynamik der Solvatation im angeregten Zustand:

$$
S(t)=\frac{\Delta E(t)-\Delta E(t=\infty)}{\Delta E(t=0)-\Delta E(t=\infty)},
$$

wobei $\Delta E(t)$ die Lage des Spektrums in einer zur Energie proportionalen Einheit, meist in $\mathrm{cm}^{-1}$, bezeichnet. Als Lage des Spektrums eignet sich die Position des Emissionsmaximums. Bei Emissionsspektren, die während der Solvatation allerdings eine starke Änderung ihrer Bandenform zeigen, hat es sich als günstiger erwiesen als Kriterium der spektralen Position die ersten Momente, d.h. deren Schwerpunkte, heranzuziehen.

Die Solvatationsdynamik von Wasser an der Lipidmembran/Wasser-Grenzschicht in unilamellaren Vesikeln wurde in dieser Arbeit über die Technik des dynamischen Stokes-Shifts bestimmt. Für die Langzeitdynamik wurde das zeitkorrelierte 
Photonenzählen angewendet (siehe Kapitel 4.2), während für die schnelleren Dynamiken das Fluoreszenzkonversionsexperiment herangezogen wurde (siehe Kapitel 4.4). Die verwendeten Farbstoffsonden sollten für diese Techniken sowohl eine starke Fluoreszenz als auch einen möglichst großen Stokes-Shift aufweisen. Hier wurde der Farbstoff Laurdan verwendet, der sich zudem durch eine definierte Lokalisierung an der Grenzschicht gegenüber anderen Farbstoffen auszeichnet (siehe Kapitel 3.5.1).

\subsection{Photon-Echo-Spektroskopie}

Ein ganz anderer Ansatz zur Untersuchung der Solvatationsdynamik ergibt sich durch das Studium der Gleichgewichtsfluktuationen, welche beispielsweise auch zur Verbreiterung des linearen Absorptionsspektrums führen. Im Grenzfall einer kleinen Störung der Ladungsdichteverteilung beim Übergang $|\mathrm{e}\rangle \rightarrow|\mathrm{g}\rangle$ sind dynamischer Stokes-Shift und die Verbreiterung des linearen Absorptionsspektrums nicht unabhängig voneinander. Die lineare Antworttheorie geht davon aus, daß die Zeitskala, auf der die thermischen Fluktuationen im Gleichgewicht stattfinden, ebenso die Zeitskala definiert, mit der das Nichtgleichgewichtssystem im angeregten Zustand relaxiert. Das bedeutet, daß die Formen der System-BadWechselwirkungspotentiale im Grund- und angeregtem Zustand identisch sind und sich lediglich in der Gleichgewichtskernlage der Solvatationskoordinate $q$ unterscheiden [27].

Die zur Verbreiterung führenden Gleichgewichtsfluktuationen $\delta \omega_{\text {eg }}(t)$ der elektronischen Energielücke $\omega_{\mathrm{eg}}(t)$, werden durch die System-Bad-Korrelationsfunktion $M(t)$ beschrieben:

$$
M(t)=\frac{\left\langle\delta \omega_{\mathrm{eg}}(t) \delta \omega_{\mathrm{eg}}(0)\right\rangle}{\left\langle\delta \omega_{\mathrm{eg}}^{2}\right\rangle} .
$$

Bei Gültigkeit der linearen Antworttheorie sind $M(t)$ und $S(t)$ proportional zueinander und erlauben damit einen grundsätzlich anderen Ansatz zum Studium der dynamischen Solvatation. Diese Proportionalität wurde von Mukamel im Modell der Brown'schen Oszillatoren anschaulich gezeigt [18]. Bei genauer Kenntnis von $M(t)$ können prinzipiell alle linearen und nichtlinearen spektroskopischen Observablen vorhergesagt werden.

Eine erste Abschätzung von $M(t)$ kann durch das konventionelle, d. h. zeitintegrierte 3-Puls Photon-Echo Peakshift Experiment (3PEPS) erhalten werden. Es wurde gezeigt, daß der beobachtete Echo-Peakshift direkt mit dem Verhältnis zwischen homogener und inhomogener Linienverbreiterung im System in Beziehung gesetzt werden kann $[23,28,29,30,31,32,33,34]$. Für sehr frühe Zeiten ist die direkte Beziehung zwischen beobachtetem Peakshift und Solvatationsdynamik allerdings nicht mehr gegeben. Hier müssen weiterreichende Techniken, wie z. B. das zeitaufgelöste 3-Puls Photon-Echo Experiment, herangezogen werden [28, 29,35]. 
Im folgenden wird zunächst eine kurze Einführung in die Vierwellenmischtechniken (4WM, Four-Wave-Mixing) gegeben, zu denen das Photon-EchoExperiment zählt. Anschließend wird das zeitintegrierte 3-Puls Photon-Echo Peakshift Experiment (3PEPS) eingehend behandelt. Dabei folgt die Beschreibung weitestgehend der Beschreibung von de Boeij et al. [29,36]. Ebenso wird kurz auf das ebenfalls in der vorliegenden Arbeit angewendete Transient-GratingExperiment (TG) eingegangen.

\subsubsection{Vier-Wellen-Mischprozesse}

Die störungstheoretische Beschreibung von Vier-Wellen-Mischprozessen (4WM) ist in der Literatur ausführlich beschrieben [18,37]. Die Pulssequenz des stimulierten Photon-Echos ist in Abbildung 2.3 dargestellt. Der erste Puls $\vec{k}_{1}$ erzeugt eine Kohärenz zwischen Grund- und angeregtem Zustand des verwendeten Chromophors. Der zweite Puls $\vec{k}_{2}$ beendet diese Kohärenzperiode $t_{12}$ und startet die sogenannte Populationsperiode $t_{23}$ (im Grund- oder angeregten Zustand), in welcher spektrale Diffusion stattfindet. Der letzte Puls $\vec{k}_{3}$ beendet auch diese Periode und startet eine zweite Kohärenzperiode. Daraufhin wird eine Polarisation dritter Ordnung, d.h. das Echo-Signal in die Raumrichtung entsprechend der Phasenanpassung $\vec{k}_{2}+\vec{k}_{3}-\vec{k}_{1}$ gemäß der Impulserhaltung abgestrahlt [38]. Die zur störungstheoretischen Beschreibung des Photon-Echos notwendigen doppelseitigen Feynman-Diagramme sind in Abbildung 2.4 dargestellt. Eine umfassende Behandlung der Theorie mit Hilfe von Liouville-Pfaden und Feynman-Diagrammen bietet Mukamel [18].

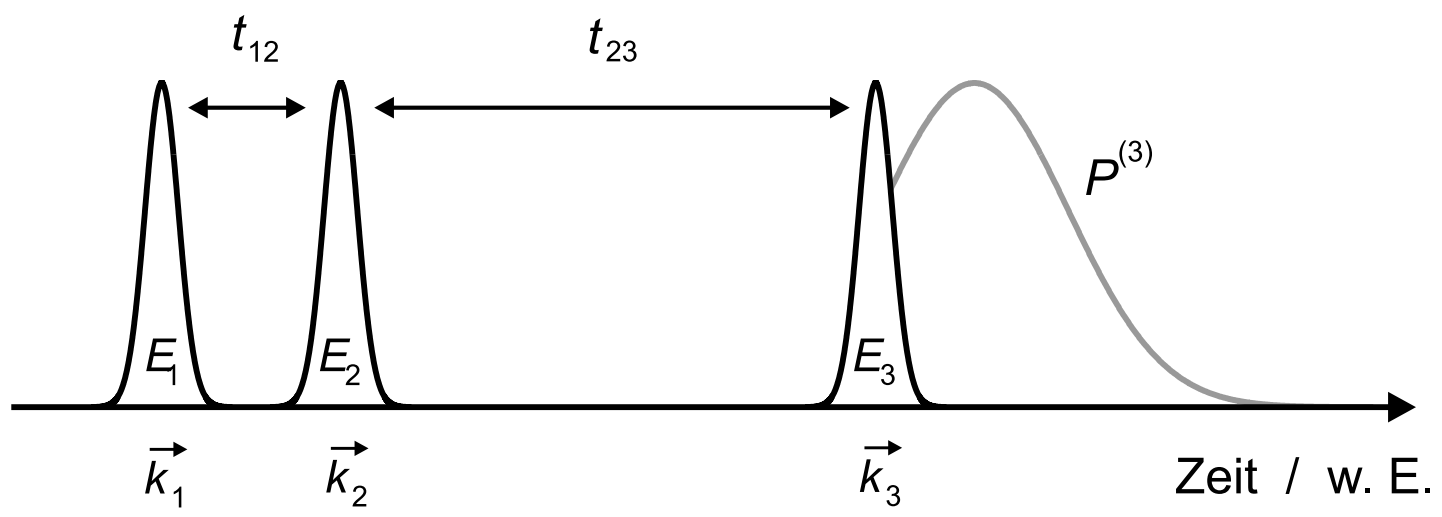

Abbildung 2.3: Pulssequenz für das zeitintegrierte 3-Puls Photon-Echo Peakshift Experiment mit relevanten Verzögerungszeiten $t_{12}$ und $t_{23}$ zwischen den eingestrahlten Feldern $E_{1}-E_{3}$ mit den Wellenvektoren $\vec{k}_{1}-\vec{k}_{3}$. 


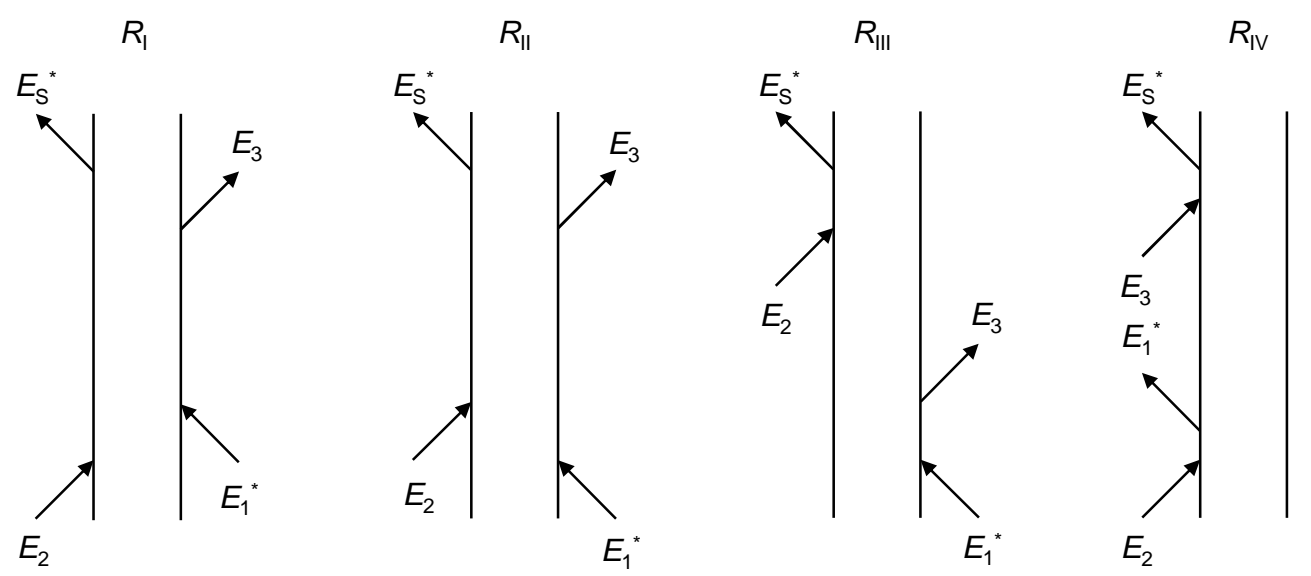

Abbildung 2.4: Feynman-Diagramme für die störungstheoretische Beschreibung der Polarisation dritter Ordnung $P^{(3)}$ in Richtung $\vec{k}_{3}+\vec{k}_{2}-\vec{k}_{1}$ in einem Zweiniveausystem mit Grundzustand $|g\rangle$ und angeregtem Zustand $|e\rangle$. Die Diagramme II und III beschreiben das reale Echo, während I und IV das sogenannte virtuelle Echo beschreiben.

In einem Zweiniveausystem gilt in RWA-Näherung (RWA, Rotating-Wave Approximation) für die in die Richtung $\vec{k}_{3}+\vec{k}_{2}-\vec{k}_{1}$ abgestrahlte nichtlineare Polarisation dritter Ordnung:

$$
\begin{aligned}
P^{(3)}\left(t, t_{12}, t_{23}\right) \propto & \left(\frac{i}{\hbar}\right)^{3} \exp \left(-\frac{t_{23}}{T_{1}}\right) \int_{0}^{\infty} \mathrm{d} t_{3} \int_{0}^{\infty} \mathrm{d} t_{2} \int_{0}^{\infty} \mathrm{d} t_{1} \\
& \left\{\left[R_{I I}\left(t_{3}, t_{2}, t_{1}\right)+R_{I I I}\left(t_{3}, t_{2}, t_{1}\right)\right] \times E_{3}\left(t-t_{3}\right) E_{2}\left(t+t_{23}-t_{3}-t_{2}\right)\right. \\
& E_{1}^{*}\left(t+t_{12}+t_{23}-t_{3}-t_{2}-t_{1}\right) \exp \left[-i\left(\omega_{\mathrm{eg}}-\omega\right)\left(t_{3}-t_{1}\right)\right]+ \\
& {\left[R_{I}\left(t_{3}, t_{2}, t_{1}\right)+R_{I V}\left(t_{3}, t_{2}, t_{1}\right)\right] \times E_{3}\left(t-t_{3}\right) E_{2}\left(t+t_{23}-t_{3}-t_{2}-t_{1}\right) } \\
& \left.E_{1}^{*}\left(t+t_{12}+t_{23}-t_{3}-t_{2}\right) \exp \left[-i\left(\omega_{\mathrm{eg}}-\omega\right)\left(t_{3}+t_{1}\right)\right]\right\}+ \\
& \left(\frac{i}{\hbar}\right)^{3} \exp \left(-\frac{t_{23}}{T_{1}}\right) \int_{0}^{\infty} \mathrm{d} t_{3} \int_{0}^{\infty} \mathrm{d} t_{2} \int_{0}^{\infty} \mathrm{d} t_{1} \\
& \left\{\left[R_{I I}\left(t_{3}, t_{2}, t_{1}\right)+R_{I I I}\left(t_{3}, t_{2}, t_{1}\right)\right] \times E_{3}\left(t+t_{23}-t_{3}-t_{2}\right) E_{2}\left(t-t_{3}\right)\right. \\
& E_{1}^{*}\left(t+t_{12}+t_{23}-t_{3}-t_{2}-t_{1}\right) \exp \left[-i\left(\omega_{\mathrm{eg}}-\omega\right)\left(t_{3}-t_{1}\right)\right]+ \\
& {\left[R_{I}\left(t_{3}, t_{2}, t_{1}\right)+R_{I V}\left(t_{3}, t_{2}, t_{1}\right)\right] \times E_{3}\left(t+t_{23}-t_{3}-t_{2}-t_{1}\right) E_{2}\left(t-t_{3}\right) } \\
& \left.E_{1}^{*}\left(t+t_{12}+t_{23}-t_{3}-t_{2}\right) \exp \left[-i\left(\omega_{\mathrm{eg}}-\omega\right)\left(t_{3}+t_{1}\right)\right]\right\} .
\end{aligned}
$$


Darin entspricht $\omega_{\text {eg }}$ der Frequenz des 0-0-Übergangs der elektronischen Energielücke, welche aus dem ersten Moment des linearen Absorptionsspektrums bestimmt werden kann. $\omega$ beschreibt die Frequenz der eingestrahlten Felder $E_{1}-E_{3}$ mit den Wellenvektoren $\vec{k}_{1}-\vec{k}_{3}$ und $t$ die Zeit nach Einstrahlung des dritten Pulses. $T_{1}$ bezeichnet die Relaxationszeit der erzeugten Population. Die nichtlinearen Antwortfunktionen dritter Ordnung $R_{I}-R_{I V}$ werden durch sogenannte Kumulanten-Entwicklung erhalten:

$$
\begin{aligned}
& R_{I}\left(t_{3}, t_{2}, t_{1}\right)=\exp \left[-g^{*}\left(t_{3}\right)-g\left(t_{1}\right)-g^{*}\left(t_{2}\right)+\right. \\
& \left.g^{*}\left(t_{2}+t_{3}\right)+g\left(t_{1}+t_{2}\right)-g\left(t_{1}+t_{2}+t_{3}\right)\right], \\
& R_{I I}\left(t_{3}, t_{2}, t_{1}\right)=\exp \left[-g^{*}\left(t_{3}\right)-g^{*}\left(t_{1}\right)+g\left(t_{2}\right)-\right. \\
& \left.g\left(t_{2}+t_{3}\right)-g^{*}\left(t_{1}+t_{2}\right)+g^{*}\left(t_{1}+t_{2}+t_{3}\right)\right], \\
& R_{I I I}\left(t_{3}, t_{2}, t_{1}\right)=\exp \left[-g\left(t_{3}\right)-g^{*}\left(t_{1}\right)+g^{*}\left(t_{2}\right)-\right. \\
& \left.g^{*}\left(t_{2}+t_{3}\right)-g^{*}\left(t_{1}+t_{2}\right)+g^{*}\left(t_{1}+t_{2}+t_{3}\right)\right], \\
& R_{I V}\left(t_{3}, t_{2}, t_{1}\right)=\exp \left[-g\left(t_{3}\right)-g\left(t_{1}\right)-g\left(t_{2}\right)+\right. \\
& \left.g\left(t_{2}+t_{3}\right)+g\left(t_{1}+t_{2}\right)-g\left(t_{1}+t_{2}+t_{3}\right)\right] .
\end{aligned}
$$

Dabei beschreiben die Antwortfunktionen $R_{I I}$ und $R_{I I I}$ das konventionelle Photon-Echo in einem inhomogen verbreiterten System [39]. Die beiden anderen Antwortfunktionen $R_{I}$ und $R_{I V}$ beschreiben das sogenannte virtuelle Echo, welches unabhängig von den zugrundeliegenden Verbreiterungsmechanismen stets bei $t=0$ maximal ist [40].

In der Beschreibung der Vierwellen-Mischprozesse spielt die sogenannte Linienverbreiterungsfunktion $g(t)$ eine zentrale Rolle und ist mit den Hilfskorrelationsfunktionen $M^{\prime}(t)$ und $M^{\prime \prime}(t)$ wie folgt verknüpft [18]:

$$
g(t)=\Delta^{2} \int_{0}^{t} \mathrm{~d} \tau_{1} \int_{0}^{\tau_{1}} \mathrm{~d} \tau_{2} M^{\prime}\left(\tau_{2}\right)-i \lambda \int_{0}^{t} \mathrm{~d} \tau\left[1-M^{\prime \prime}(\tau)\right]
$$


Die Hilfskorrelationsfunktionen $M^{\prime}(t)$ bzw. $M^{\prime \prime}(t)$ und die beiden statischen Kopplungsstärkeparameter $\Delta$ bzw. $\lambda$ lassen sich mit der spektralen Dichte $C(\omega)$ in Beziehung setzen:

$$
\begin{aligned}
M^{\prime}(t) & =\frac{1}{\Delta^{2}} \int_{0}^{\infty} \mathrm{d} \omega C(\omega) \operatorname{coth}\left(\frac{\hbar \omega}{2 k_{\mathrm{B}} T}\right) \cos \omega t, \\
M^{\prime \prime}(t) & =\frac{1}{\lambda} \int_{0}^{\infty} \mathrm{d} \omega \frac{C(\omega)}{\omega} \cos \omega t, \\
\Delta^{2} & =\int_{0}^{\infty} \mathrm{d} \omega C(\omega) \operatorname{coth}\left(\frac{\hbar \omega}{2 k_{\mathrm{B}} T}\right), \\
\lambda & =\int_{0}^{\infty} \mathrm{d} \omega \frac{C(\omega)}{\omega} .
\end{aligned}
$$

Darin ist $k_{\mathrm{B}}$ die Boltzmannkonstante und $T$ die Temperatur. Die Gleichungen 2.6 zeigen, daß die spektrale Dichte $C(\omega)$ die zentrale Größe bei der Beschreibung des Vierwellenmischens im Formalismus der Kumulanten-Entwicklung ist. Bei Kenntnis von $C(\omega)$ können alle anderen Größen direkt berechnet werden. Die Solvatationskorrelationsfunktion $S(t)$, wie sie beispielsweise im zeitabhängigen Stokes-Shift Experiment gemessen wird, steht in direkter Beziehung zu $M^{\prime \prime}(t)$, während das Photon-Echo-Experiment sensitiv auf $M^{\prime}(t)$ ist.

Die Hilfskorrelationsfunktionen $M^{\prime}(t)$ und $M^{\prime \prime}(t)$ sind allerdings nicht unabhängig voneinander, sondern stehen mit dem Real- und Imaginärteil der Korrelationsfunktion $M(t)=\operatorname{Re}[M(t)]+i \operatorname{Im}[M(t)]$ in direkter Beziehung:

$$
\begin{aligned}
& M^{\prime}(t)=\frac{1}{\Delta^{2}} \operatorname{Re}[M(t)] \quad \text { bzw. } \\
& M^{\prime \prime}(t)=1+\frac{1}{\lambda} \int_{0}^{t} \mathrm{~d} \tau \operatorname{Im}[M(t)] .
\end{aligned}
$$

Weil der Real- und Imaginärteil von $M(t)$ über das Fluktuations-DissipationsTheorem miteinander verknüpft sind, lassen sich die beiden Hilfskorrelationsfunktionen $M^{\prime}(t)$ und $M^{\prime \prime}(t)$ mit Hilfe einer Cosinus-Transformation gefolgt von einer inversen Cosinus-Transformation ineinander überführen [18]:

$$
M^{\prime}(t)=\frac{\lambda}{\pi \Delta^{2}} \int_{0}^{\infty} \mathrm{d} \omega \cos (\omega t) \omega \operatorname{coth}\left(\frac{\hbar \omega}{2 k_{\mathrm{B}} T}\right) \int_{0}^{\infty} \mathrm{d} \tau \cos (\omega \tau) M^{\prime \prime}(\tau) .
$$

Für die eingeführten Hilfskorrelationsfunktionen gilt darüberhinaus $M^{\prime}(0)=$ $M^{\prime \prime}(0)=1$ und $\dot{M}^{\prime}(0)=\dot{M}^{\prime \prime}(0)=0$.

Die eingeführten Kopplungsparameter $\Delta$ und $\lambda$ lassen sich weiterhin mit physikalischen Eigenschaften des Systems verknüpfen. Dazu werden Ausdrücke für 
die Linienform von Absorption und Emission aus der Fouriertransformierten der Linienformfunktion $g(t)$ erhalten:

$$
\begin{aligned}
& \sigma_{\mathrm{Abs}}(\omega) \propto \operatorname{Re} \int_{0}^{\infty} \mathrm{d} t \exp \left[i\left(\omega-\omega_{e g}\right) t-g(t)\right] \quad \text { bzw. } \\
& \sigma_{\mathrm{Emi}}(\omega) \propto \operatorname{Re} \int_{0}^{\infty} \mathrm{d} t \exp \left[i\left(\omega-\omega_{e g}+2 \lambda\right) t-g^{*}(t)\right] .
\end{aligned}
$$

Daraus folgt, daß das mit dem Realteil der Linienformfunktion verknüpfte $\Delta$ die Breite von Absorptions- und Emissionsspektren repräsentiert, wohingegen $2 \lambda$ den Stokes-Shift darstellt und mit der Solvatationsreorganisationsenergie in Verbindung gebracht wird.

\subsubsection{3-Puls Photon-Echo Peakshift}

Im zeitintegrierten 3-Puls Photon-Echo Peakshift Experiment (3PEPS) wird das Maximum des stimulierten Photon-Echos (SPE) für eine feste Verzögerung $t_{23}$ zwischen zweiten und drittem Anregungspuls in Abhängigkeit von $t_{12}$ gemessen:

$$
I_{\mathrm{SPE}}\left(t_{23}, t_{12}\right)=\int_{0}^{\infty}\left|P^{(3)}\left(t, t_{23}, t_{12}\right)\right|^{2} \mathrm{~d} t
$$

Der Peakshift wird dabei bezüglich einer Nullpunktsreferenz bestimmt, für die $t_{12}=0$ gilt. Der Peakshift in Abhängigkeit von $t_{23}$ wird dann als Peakshiftfunktion $S\left(t_{23}\right)$ bezeichnet.

Es wurde von de Boeij et al. gezeigt, daß die Peakshiftfunktion $S\left(t_{23}\right)$ in engem Zusammenhang zur System-Bad-Korrelationsfunktion $M(t)$ steht [30]. Flüssigkeiten lassen sich oftmals mit einer Korrelationsfunktion $M^{\prime}(t)$ beschreiben, die sich in einen schnellen und einen langsamen Anteil aufteilen läßt:

$$
M^{\prime}(t)=(1-a) M_{\text {Schnell }}^{\prime}(t)+a M_{\text {Langsam }}^{\prime}(t) \quad \text { mit } \quad 0 \leq a \leq 1 .
$$

Der schnelle Anteil von $M^{\prime}(t)$ resultiert aus einer Kombination von Wellenpaketdynamik und inertialer Solvatation. Damit die Peakshift-Funktion die Korrelationsfunktion beschreibt muß vorausgesetzt sein, daß der schnelle Anteil in der Zeit $t_{23}$ auf Null abfällt, d. h. $M_{\text {Schnell }}^{\prime}\left(t_{23}\right)=0$. Gleichzeitig muß der langsame Anteil von $M^{\prime}(t)$ auf den Zeitskalen von $t$ und $t_{12}$ stationär erscheinen. Dann läßt sich folgender Ausdruck ableiten:

$$
\begin{aligned}
I_{\mathrm{SPE}}\left(t_{23}, t_{12}\right) \propto & \exp \left(-\frac{2 t_{23}}{T_{1}}\right) \exp \left[-\Delta^{2}{t_{12}}^{2}\left(1-a^{2} M_{\text {Langsam }}^{\prime}\right)\right] \times \\
& \left\{\frac{\sqrt{\pi}}{2}+\operatorname{erf}\left[\Delta a M_{\text {Langsam }}^{\prime}\left(t_{23}\right) t_{12}\right]\right\} .
\end{aligned}
$$


Darin bezeichnet $\operatorname{erf}(x)$ die Fehlerfunktion. Um die Position des Echo-Maximums zu erhalten, wird diese Gleichung nach $t_{12}$ abgeleitet und gleich Null gesetzt. Es ergibt sich:

$$
\begin{aligned}
& a M_{\text {Langsam }}^{\prime}\left(t_{23}\right) \exp \left[-\Delta^{2} a^{2} M_{\text {Langsam }}^{\prime}{ }^{2}\left(t_{23}\right) S\left(t_{23}\right)^{2}\right]-\Delta S\left(t_{23}\right) \times \\
& {\left[1-a^{2} M_{\text {Langsam }}^{\prime}{ }^{2}\left(t_{23}\right)\right]\left\{\sqrt{\pi}+2 \operatorname{erf}\left[\Delta a M_{\text {Langsam }}^{\prime}\left(t_{23}\right) S\left(t_{23}\right)\right]\right\}=0 .}
\end{aligned}
$$

Darin bezeichnet die Peakshiftfunktion $S\left(t_{23}\right)$ die Position des Echo-Maximums in Abhängigkeit von $t_{23}$. Unter der Annahme, daß der schnelle Anteil der Korrelationsfunktion dominiert, ergibt sich unter ausschließlicher Berücksichtigung der linearen Termen von $a$ :

$$
a M_{\text {Langsam }}^{\prime}\left(t_{23}\right)=\frac{\Delta \sqrt{\pi} S\left(t_{23}\right)}{1+2 \Delta^{2} S^{2}\left(t_{23}\right)} .
$$

Diese Gleichung belegt, daß eine direkte Beziehung zwischen dem langsamen Anteil der System-Bad-Korrelationsfunktion $M_{\text {Langsam }}^{\prime}(t)$ und der Peakshiftfunktion $S\left(t_{23}\right)$ existiert. Im Hochtemperaturlimit und unter Voraussetzung, daß $\Delta^{2} S^{2}\left(t_{23}\right) \ll 1$ gilt, folgt weiterhin:

$$
M_{\text {Langsam }}\left(t_{23}\right)=M_{\text {Langsam }}^{\prime}\left(t_{23}\right)=M_{\text {Langsam }}^{\prime \prime}\left(t_{23}\right)=\frac{\Delta \sqrt{\pi}}{a} S\left(t_{23}\right) .
$$

Es zeigt sich, daß im Hochtemperaturlimit eine direkte Proportionalität zwischen der Peakshiftfunktion und dem langsamen Teil der System-BadKorrelationsfunktion besteht. Dabei ist zu beachten, daß die Peakshiftfunktion die Korrelationsfunktion unabhängig von der Populationsrelaxationszeit $T_{1}$ beschreibt.

Zu frühen Zeiten existiert jedoch eine Diskrepanz zwischen Peakshiftfunktion und Korrelationsfunktion. Dies läßt sich instruktiv durch die anfängliche Steigung des Echo-Peakshifts bei $t_{23}=0$ zeigen. Sie erweist sich stets als ungleich Null, wie von de Boeij etal. gezeigt wurde [29]. Da es sich beim schnellen Teil der Korrelationsfunktion jedoch um inertiale Bewegungen handelt, muß die Steigung der Korrelationsfunktion dort Null sein. Daraus folgt unmittelbar, daß die Peakshiftfunktion nicht die Kurzzeitdynamik der Korrelationsfunktion wiederzugeben vermag. Der genaue Punkt, an dem $S\left(t_{23}\right)$ eine gute Beschreibung von $M(t)$ liefert, läßt sich allerdings nur schwer feststellen. Um eine exakte Beschreibung von $M(t)$ zu allen Zeiten zu erhalten, muß beispielsweise auf das zeitaufgelöste Photon-Echo Experiment zurückgegriffen werden [28, 29,35].

Abschließend läßt sich festhalten, daß das zeitintegrierte Photon-Echo Peakshift Experiment mit Ausnahme ultraschneller Zeitkomponenten in der Lage ist, die System-Bad-Korrelationsfunktion $M(t)$ wiederzugeben. Diese Beziehung und 
die einfache experimentelle Umsetzung macht die Photon-Echo Spektroskopie zu einem nützlichen Werkzeug zur Bestimmung der Solvatationsdynamik in Lösung. Sie stellt eine leistungsfähige Alternative zur Bestimmung der Solvatationsdynamik mit Hilfe des zeitabhängigen Fluoreszenz Stokes-Shifts dar und wird in der vorliegenden Arbeit zur Bestimmung der Solvatation in inversen Mizellen verwendet (siehe Kapitel 6). Bei der Suche nach einer geeigneten Farbstoffsonde erweist es sich hier als vorteilhaft, daß der gesuchte Chromophor keinen großen Stokes-Shift aufzuweisen braucht. Daher wurde der Farbstoff HIDCI verwendet, welcher sich durch einen extrem großen Extinktionskoeffizienten auszeichnet (siehe Kapitel 3.5.2).

\subsubsection{Transient-Grating-Spektroskopie}

Die Erzeugung optischer Kohärenzen im Femtosekundenbereich führt oftmals zur Bildung von Wellenpaketen. Deren Dynamik gibt unter anderem die Schwingungsstruktur des angeregten Chromophors wieder. Solche Schwingungen überlagern beispielsweise das Signal des Photon-Echo Peakshifts und können im Modell der Brown'schen Oszillatoren durch schwach gedämpfte Moden wiedergegeben werden. Während die Oszillationen auf der Peakshift-Funktion nur schwer interpretierbar sind, stellt die Transient-Grating-Spektroskopie (TG) eine Methode dar, um die Wellenpaketdynamik eingehend zu studieren. Einen guten Überblick der Möglichkeiten dieser Technik geben Joo et al. [41] und Brown et al. [42].

Das Transient-Grating-Experiment unterscheidet sich vom 3-Puls Photon-Echo Experiment lediglich darin, daß die beiden ersten Felder $E_{1}$ und $E_{2}$ stets zeitgleich in die Probe eingestrahlt werden, d. h. $t_{12}=0$. In einem einfachen physikalischen Bild erzeugen die beiden Felder durch Interferenz ein transientes Gitter in der zu untersuchenden Probe, welches durch Relaxationsprozesse nach Einstrahlung wieder zerfällt. Bei Einstrahlung eines dritten Feldes $E_{3}$ nach einer Verzögerungszeit $t_{23}$ kommt es zur Beugung an diesem Gitter in die Raumrichtung $\vec{k}_{2}+\vec{k}_{3}-\vec{k}_{1}$. Ist die Bandbreite der Laserpulse größer als der Energieabstand benachbarter Schwingungszustände bzw. die Pulsdauer kürzer als eine Schwingungsperiode, so wird ein kohärentes Wellenpaket erzeugt. Die Dynamik des Wellenpakets führt zu einer Modulation des gebeugten Feldes, was sich durch ausgeprägte Oszillationen auf dem Transient-Grating-Signal bemerkbar macht.

Die Observable des TG-Experimentes läßt sich wiederum durch das zeitintegrierte Betragsquadrat der Polarisation dritter Ordnung ausdrücken:

$$
I_{\mathrm{TG}}\left(t_{23}\right)=\int_{0}^{\infty}\left|P^{(3)}\left(t_{12}=0, t_{23}, t\right)\right|^{2} \mathrm{~d} t
$$


Nach Brown et al. läßt sich das TG-Signal in folgender Form darstellen [42]:

$$
I_{\mathrm{TG}}\left(t_{23}\right)=\left|A \exp \left(-\frac{t_{23}^{2}}{\Gamma^{2}}\right)+\sum_{i=1}^{3 n-5 \text { oder } 3 n-6} B_{i} \cos \left(\omega_{i} t_{23}+\phi_{i}\right)+C R_{\text {Rotation }}\right| .
$$

Darin wird dem sogenannten Kohärenz-Artefakt, einem Peak nahe $t_{23}=0$, durch eine Gaußfunktion der Breite $\Gamma$ und der Amplitude $A$ Rechnung getragen. Die weiteren Terme beschreiben Signalbeiträge, die aus Schwingungen und Rotationen des untersuchten Moleküls resultieren. $B_{i}$ beschreibt die Amplitude der betrachteten $i$-ten Schwingung der Frequenzen $\omega_{i}$ und der Phase $\phi_{i}$. Die Anzahl möglicher Schwingungen in einem Molekül mit $n$ Atomen ist dabei $3 n-5$ für lineare bzw. $3 n-6$ für gewinkelte Moleküle. Rotationen der Amplitude $C$ werden mit dem verallgemeinerten Term $R_{\text {Rotation }}$ berücksichtigt.

In der vorliegenden Arbeit wurde die Schwingungsstruktur des verwendeten Farbstoffes HIDCI in reinem Wasser und in inversen Mizellen bestimmt (siehe Kapitel 6.4). 


\section{Kapitel 3}

\section{Biologische Modellsysteme}

Eine Vielzahl biologischer Moleküle, wie z. B. Proteine, Steroide und insbesondere Phospholipide, besitzen amphiphilen Charakter. In Wasser bilden solche Moleküle spontan typische Molekülanhäufungen, sogenannte Aggregate, aus. Hydrophobe und hydrophile Wechselwirkungen zwischen den Molekülen liefern die thermodynamische Triebkraft zur Bildung und Aufrechterhaltung der Aggregatstrukturen durch Minimierung der Energie des Gesamtsystems. Aufgrund ihrer Fähigkeit komplexe Strukturen in Wasser auszubilden, werden sie als selbstorganisierende Systeme bezeichnet $[9,43]$.

Abbildung 3.1 zeigt schematisch drei typische Aggregatstrukturen. Mizellen stellen deren einfachste Vertreter dar und bilden sich oberhalb einer sogenannten kritischen Mizellenkonzentration in Wasser spontan aus. Bekannt ist die Bildung von Mizellen aus Seifenmolekülen und anderen Detergentien, aber auch in der Natur werden aus Lipiden bestehende Mizellen angetroffen. Die bekanntesten Vertreter von Molekülaggregaten in der Natur sind jedoch die aus Lipid-Doppelschichten bestehenden Membranen. Sie dienen zur Abgrenzung von Zellen und Zellorganellen und stellen darüberhinaus eine Matrix für eine Vielzahl unterschiedlichster Proteine dar. Bei der Fusion von Membranen können sich unter gewissen Voraussetzungen Strukturen ausbilden, die Wasser in einer Art inversen Mizelle einschließen [43]. Eine andere Art von Wassereinschluß ergibt sich, wenn sich eine ausgedehnte Lipid-Doppelschicht zu einer Hohlkugel schließt. Solche Strukturen werden als Liposome oder Vesikel bezeichnet. Die ersten lebenden Zellen ähnelten vermutlich solchen Vesikeln, deren wäßriger Inhalt durch die hydrophobe Hülle von der Außenwelt getrennt war. Das Studium der aus amphiphilen Molekülen bestehenden Aggregatstrukturen nimmt eine zentrale Stellung in der modernen Biophysik ein, was aus ihrer nicht zu unterschätzenden Relevanz in der Natur resultiert $[9,43]$. 

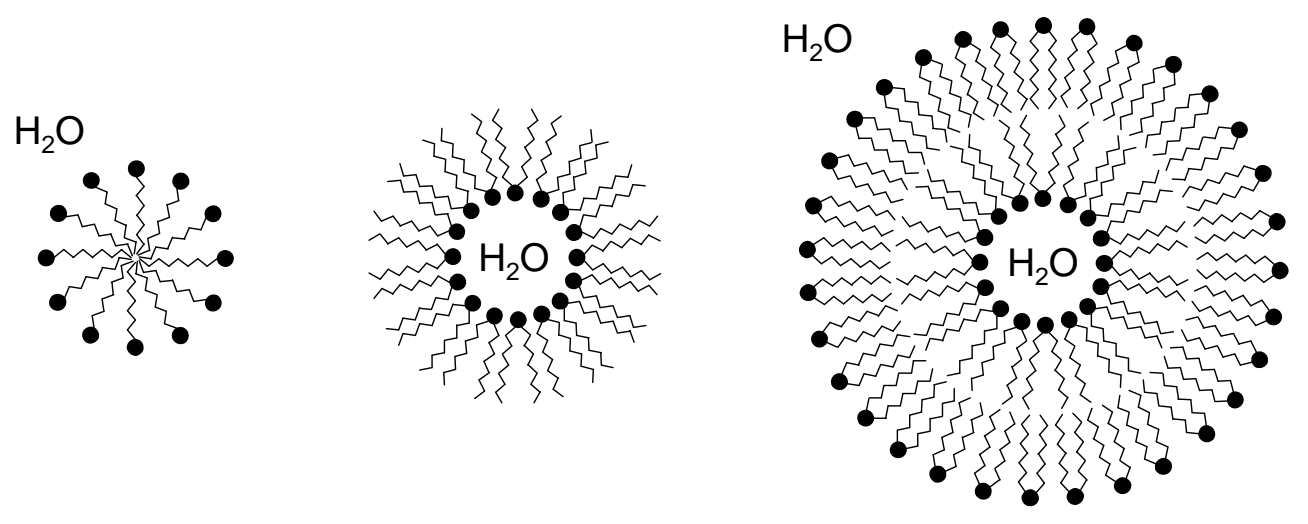

Abbildung 3.1: Verschiedene Aggregate amphiphiler Moleküle in Wasser. Links: Mizelle; Mitte: Inverse Mizelle; Rechts: Vesikel.

Auch wenn die biologischen Funktionen der Molekülaggregate untereinander durchaus verschieden sind, so haben sie dennoch die Gemeinsamkeit, daß sich ihre unmittelbare Wasserumgebung von der des reinen Wassers unterscheidet. In der vorliegenden Arbeit soll daher die Solvatationsdynamik von Wasser an typischen Grenzschichten biologischer Modellsysteme studiert werden. Auch wenn die in der Natur vorkommenden Verhältnisse weitaus komplexer als die hier untersuchten sind, so sollten dennoch prinzipielle Erkenntnisse für die Veränderung der Solvatationsdynamik an biologischen Grenzschichten und damit einhergehend des Wasserstoffbrückennetzwerkes gewonnen werden können.

In den folgenden Kapiteln sollen die in Abbildung 3.1 dargestellten Modellsysteme bezüglich ihrer physikalischen Eigenschaften charakterisiert werden. In diesem Rahmen wird insbesondere auf Arbeiten zur Solvatationsdynamik in solchen Systemen eingegangen, wobei die wichtigsten Erkenntnisse kurz beschrieben werden. Um die gefundenen Dynamiken von Wasser in eingeschränkten Systemen einordnen zu können, werden zunächst Ergebnisse an reinem Wasser vorgestellt.

\subsection{Wasser}

Die der Solvatation zugrundeliegenden Dynamiken wurden in den letzten Jahrzehnten in einer Vielzahl von Flüssigkeiten eingehend untersucht [44]. Wasser stellt dabei aufgrund seiner biologischen Relevanz und seiner einzigartigen Eigenschaften die interessanteste aller Flüssigkeiten dar. Daher ist die Untersuchung der dynamischen und strukturellen Eigenschaften von Wasser eines der lebendigsten Forschungsgebiete der physikalischen Chemie der letzten Jahrzehnte, sowohl in experimenteller $[12,24,38,45,46,47,48,49,50,51,52,53,54,55,56,57,58,59,60]$ als auch in theoretischer Hinsicht $[58,61,62,63,64,65,66,67,68,69,70,71,72$, 
$73,74,75,76,77]$. Hervorzuheben sind Experimente in der Zeitdomäne, wie die Messung des optischen Kerr-Effektes (OKE) [45, 46, 47, 48], des zeitabhängigen Stokes-Shifts (TDFSS) [24,49] und des Photon-Echo Peakshifts (3PEPS) [38,50]. Desweiteren wurde die THz-Spektroskopie zur Untersuchung der Wasserdynamik herangezogen [51,52]. Experimente in der Frequenzdomäne umfassen vorrangig die Raman- $[53,54,55]$ und die IR-Spektroskopie $[56,57,58,59,60]$. Die Bestimmung der Solvatationsdynamik über Fluoreszenzkonversionsexperimente [24, 49] und Photon-Echo-Techniken [38,50] steht dabei im Hinblick auf die in dieser Arbeit präsentierten Techniken im Mittelpunkt des Interesses.

Da Wasser die schnellste aller bisher in Flüssigkeiten beobachteten Dynamiken aufweist, dauerte es bis zur Etablierung ultraschneller Lasertechniken bis Barbara und Mitarbeiter 1988 zum ersten Mal die ultraschnelle Solvatation in Wasser beobachten konnten [49]. Es handelte sich dabei um ein Fluoreszenzkonversionsexperiment an dem Farbstoff 7-Dimethylamino-coumarin-4-acetat. Sie fanden ein biexponentielles Abklingen der Solvatationskorrelationsfunktion mit Zeitkonstanten von $0.16 \mathrm{ps}(33 \%)$ und $1.2 \mathrm{ps}$ (67\%). Die Daten wurden mit dielektrischen Kontinuumsmodellen (DCM) [58,66], einem Mean-SphericalApproximation-Modell (MSA) [67,68] und molekulardynamischen Simulationen (MD) $[69,70,71,72]$ verglichen. Auch wenn keine quantitative Übereinstimmung mit den experimentellen Daten gefunden wurde, so konnte zumindest das biexponentielle Abklingverhalten und die Größenordnung der gefundenen Zeitkonstanten wiedergegeben werden.

In der Folgezeit wurden die Experimente mit anderen Farbstoffsonden wiederholt. So fanden Fleming und Mitarbeiter mit Coumarin 343, einer Variante von 7-Dimethylamino-coumarin-4-acetat, die eventuell auftretende Charge-TransferZustände unterbindet, einen gaußförmigen Inertialteil mit einer Zeitkonstanten um $50 \mathrm{fs}$, sowie ein biexponentielles Abklingverhalten mit Zeitkonstanten von $126 \mathrm{fs}(20 \%)$ und $880 \mathrm{fs}$ (35\%) [24]. Der Inertialteil wurde durch einen Vergleich mit molekulardynamischen Simulationen Librationsmoden einzelner Wassermoleküle zugeschrieben [71,78].

Eine Vielzahl an Experimenten und Simulationen konnten den beobachteten Inertialteil und die ermittelten Zeitkonstanten prinzipiell bestätigen. Theoretische Arbeiten sagten dabei in der Regel jedoch eine noch schnellere Dynamik voraus. So ist nach Maroncelli und Fleming [71] bzw. Bader und Chandler [73] ein ultraschneller Anteil um 25 fs zu erwarten, der zudem eine Amplitude zwischen $70 \%$ und $90 \%$ aufweisen sollte. Auch die Arbeiten von Song und Chandler [74] sagen eine schnellere Solvatation voraus, als mit Hilfe der Methoden der Fluoreszenzaufkonvertierung beobachtet werden konnte.

Daher wurde in jüngster Vergangenheit die Solvatationsdynamik von Wasser im 3-Puls Photon-Echo Peakshift Experiment untersucht. Fleming und Mitarbeiter beobachteten für den Farbstoff Eosin Y einen gaußförmigen Inertialteil mit einer 
Zeitkonstanten von $17 \mathrm{fs}(73 \%)$ und ein biexponentielles Abklingen mit $400 \mathrm{fs}$ (15\%) und $2.7 \mathrm{ps}(12 \%)$ [38]. Damit wurde wie vorhergesagt eine Zeitkonstante unterhalb von $25 \mathrm{fs}$ mit einer sehr großen Amplitude gefunden. Um jedoch die Peakshift-Funktion im Modell der Brown'schen Oszillatoren wiedergeben zu können, mußte eine zusätzliche übergedämpfte Mode mit einer Korrelationszeit von 8 ps künstlich hinzugefügt werden. Obwohl diese Diskrepanz noch nicht vollständig verstanden ist, kann sie vermutlich auf die doppelt negative Ladung des Eosin Y zurückgeführt werden, welche die wassereigene Dynamik extrem stark beeinflußt [50].

Von Vöhringer und Mitarbeitern wurde die Solvatationsdynamik des Farbstoffes HIDCI in Wasser mit der gleichen Technik untersucht [50]. Bis ca. 1 ps ergibt sich eine grobe Übereinstimmung mit den Ergebnissen von Fleming und Mitarbeitern. Auf längeren Zeitskalen sind allerdings erhebliche Abweichungen zu beobachten. Die Zeitkonstanten wurden zu 17 fs (60\%), 1 ps $(19 \%)$,7.5 ps (5\%) und 180 ps $(16 \%)$ bestimmt. In dieser Arbeit wurde keine zusätzliche Mode benötigt, um die Daten im Modell der Brown'schen Oszillatoren wiedergeben zu können [79]. Durch einen Vergleich einer Vielzahl verschiedenster Techniken konnten Vöhringer und Mitarbeiter die in Wasser beobachteten Zeitkonstanten verschiedenen Relaxationsfreiheitsgraden zuordnen [45]. Die beobachtete 1 ps Komponente wurde danach gehinderten Translationsmoden des Wassers zugeordnet, während die Zeitkonstante von 7.5 ps der Rotationsdiffusion individueller Wassermoleküle zugeschrieben werden konnte. Die Photon-Echo Peakshift Messungen wurden in der vorliegenden Arbeit mit der gleichen Farbstoffsonde wiederholt. Im Rahmen der Meßgenauigkeit wurden dabei die gleichen Zeitkonstanten gefunden. Kapitel 6.2 diskutiert die erhaltenen Zeitkonstanten im Detail.

Zusammenfassend läßt sich sagen, daß die Solvatation in Wasser eine Vielzahl an Zeitskalen aufweist, die von kleiner $50 \mathrm{fs}$ bis ca. $10 \mathrm{ps}$ reichen. Zudem sind langsamere Zeitskalen zu beobachten, welche jedoch in Wasser weit weniger zur Solvatation beitragen als die ultraschnellen Komponenten. Vermutlich lassen sie sich auf die Dynamik des verwendeten Chromophors und/oder der kollektiven Rotationsdiffusion des Wassers zurückführen.

Die Solvatation an biologischen Grenzschichten unterscheidet sich deutlich von der des freien Wassers und weist deutlich langsamere Zeitkomponenten auf, wie beispielsweise von Fleming und Mitarbeitern bei der Solvatation von Coumarin 480 und Coumarin 460 in $\gamma$-Cyclodextrinen gezeigt wurde [80]. Die Solvatation an ähnlichen Grenzschichten wird im folgenden an ausgesuchten Modellsystemen beschrieben. Einen guten Überblick zur Solvatation in biologischen Umgebungen geben eine Vielzahl an Veröffentlichungen [81,82,83,84], an die sich die folgenden Ausführungen orientieren. 


\subsection{Mizellen}

Mizellen stellen die einfachsten aus amphiphilen Molekülen bestehenden Aggregatstrukturen in Wasser dar. An diesen simplen Modellsystemen zeigen sich bereits die charakteristischen dynamischen Effekte, die mit solchen Umgebungen einhergehen. Wegen ihrer grundlegenden Bedeutung beim Studium amphiphiler Molekülaggregate soll daher im folgenden in Kürze auf Mizellen eingegangen werden, auch wenn sie nicht direkter Gegenstand der in dieser Arbeit durchgeführten Untersuchungen sind. Gute Einführungen in das Forschungsgebiet der Mizellen bieten beispielsweise Tanford [85] und Moroi [86].

\subsubsection{Struktur}

Amphiphile Moleküle bilden in Wasser oberhalb einer bestimmten Konzentration, der kritischen Mizellenkonzentration (CMC, Critical Micelle Concentration), mehr oder weniger kugelförmige Molekülaggregate aus, welche als Mizellen bezeichnet werden [81,82]. Sie variieren in der Regel zwischen Größen von 1 bis $10 \mathrm{~nm}$, wobei sich pro Mizelle zwischen 20 und 200 Moleküle zusammenfinden. Der sogenannte Kern der Mizelle ist prinzipiell frei von Wasser und besteht in der Regel aus Kohlenwasserstoffketten. Die polaren Kopfgruppen der amphiphilen Moleküle weisen nach außen, d.h. in die Wasserphase. Um die Mizelle bildet sich eine sogenannte Stern'sche Doppelschicht, welche die Kopfgruppen, eventuelle Gegenionen und „gefangene“ Wassermoleküle enthält. Zwischen dieser Schicht und der freien Wasserphase befindet sich die sogenannte Gouy-ChapmanDoppelschicht, die als diffusive Schicht beschrieben werden kann. Hier bewegen sich gelöste Gegenionen und beeinflußtes aber nicht immobilisiertes Wasser, welches als "gebunden“ bezeichnet wird. Die nicht beeinflußte Wasserphase wird „frei“ genannt. In der Literatur ist die Bezeichnung dieser verschiedenen Wasserspezies leider uneinheitlich. In dieser Arbeit wird aber durchgängig die vorgestellte Namensgebung verwendet. Um die Dicken der Doppelschichten und der Mizellen zu vermessen, wurde die Kleinwinkel-Röntgen- oder Neutronen-Streuung (SANS, Small-Angle Neutron Scattering) angewendet [87,88]. Dabei zeigte sich, daß sich die Strukturen von anionischen, kationischen und neutralen Mizellen deutlich unterscheiden.

\subsubsection{Solvatationsdynamik}

Die Dynamik der Solvatation wurde von Bhattacharyya und Mitarbeitern mit Hilfe des zeitkorrelierten Einzelphotonenzählens in einer Reihe von biologischen Modellsystemen untersucht. Gegenstand der Studien waren dabei u.a. Vesikel [89, 90, 91], inverse Mizellen [92,93] und auch Mizellen [94, 95, 96, 97]. Sie be- 
trachteten drei verschiedene Typen von Mizellen: Anionische Mizellen bestehend aus Natriumdodecylsulfat (SDS), kationische Mizellen aus Cetyltrimethylammoniumbromid (CTAB) und neutrale Mizellen aus Triton X-100 (TX-100). Als Farbstoffsonden kamen Coumarin 480 (C480) [94], 4-Aminophtalimid (4-AP) [95] und 4-Dicyanomethylen-2-methyl-6-p-dimethylaminostyryl (DCM) [96] zur Anwendung. Die stationären Emissionsspektren der Farbstoffe in Mizellen unterscheiden sich dabei stark von denen in reinem Wasser und in unpolaren Umgebungen. Dies wurde als Hinweis darauf gewertet, daß sich die Farbstoffe an der Grenzschicht zwischen Mizelle und reiner Wasserphase anlagern, d. h. in der Region der Stern'schen Doppelschicht. Die genaue Lage der Farbstoffsonde ist bei der Untersuchung heterogener Umgebungen von besonderem Interesse, da die beobachteten Dynamiken durch die unmittelbare Mikroumgebung des Chromophors bestimmt werden.

Die erhaltenen Solvatationskorrelationsfunktionen $S(t)$ konnten in der Regel biexponentiell angepaßt werden. Die Ergebnisse sind in Tabelle 3.1 zusammengefaßt.

\begin{tabular}{|c|c|c|c|c|c|c|c|}
\hline Mizelle & Farbstoff & $\boldsymbol{\tau}_{\mathbf{1}} / \mathbf{p s}$ & $\boldsymbol{A}_{\mathbf{1}}$ & $\boldsymbol{\tau}_{\mathbf{2}} / \mathbf{p s}$ & $\boldsymbol{A}_{\mathbf{2}}$ & $\langle\boldsymbol{\tau}\rangle /$ ps & Lit. \\
\hline \multirow{4}{*}{ TX100 } & C480 & 550 & 0.51 & 2400 & 0.49 & 1456 & {$[94]$} \\
& $4-\mathrm{AP}$ & 331 & 0.59 & 1270 & 0.41 & 716 & {$[95]$} \\
& DCM & 300 & 0.15 & 2440 & 0.85 & 2100 & {$[96]$} \\
\hline \multirow{4}{*}{ CTAB } & C480 & 285 & 0.40 & 600 & 0.60 & 474 & {$[94]$} \\
& $4-A P$ & 100 & 0.39 & 383 & 0.61 & 273 & {$[95]$} \\
& DCM & 170 & 0.50 & 630 & 0.50 & 400 & {$[96]$} \\
\hline \multirow{5}{*}{ SDS } & C480 & 180 & 0.74 & 2140 & 0.26 & 690 & {$[94]$} \\
& $4-A P$ & 82 & 1.00 & - & - & 82 & {$[95]$} \\
& DCM & 160 & 0.55 & 2900 & 0.45 & 1400 & {$[96]$} \\
\hline
\end{tabular}

Tabelle 3.1: Abklingverhalten der Solvatationskorrelationsfunktion in neutralen (TX100), kationischen (CTAB) und anionischen (SDS) Mizellen bestimmt durch zeitkorreliertes Einzelphotonenzählen.

Zum Vergleich der Geschwindigkeit der Solvatation wurde eine mittlere Zeitkonstante $\langle\tau\rangle$ definiert, welche sich als amplitudengewichtete Summe der einzelnen Zeitkonstanten der normierten Solvatationskorrelationsfunktionen $S(t)$ darstellt:

$$
\langle\tau\rangle=A_{1} \tau_{1}+A_{2} \tau_{2}
$$

Es zeigt sich, daß die neutralen Mizellen unabhängig vom verwendeten Farbstoff die deutlich langsamste Dynamik aufweisen. Ein eindeutiger Trend zwischen den 
ionischen Mizellen ist allerdings nicht auszumachen. Dabei ist eine ausgeprägte Abhängigkeit der Dynamik von der eingesetzten Farbstoffsonde zu beobachten. Die im Vergleich zu reinem Wasser $(\langle\tau\rangle<1$ ps nach Fluoreszenzkonversionsexperimenten) um mehr als drei Größenordnungen verlangsamte Dynamik wird mit der stark eingeschränkten Mobilität des Wassers innerhalb der Stern'schen Doppelschicht erklärt. In ihr spielen für die Solvatation der Farbstoffsonde die Kopfgruppen der amphiphilen Moleküle, eventuell vorhandene Gegenionen (SDS und CTAB) und die gebundenen Wassermoleküle eine Rolle. Die Dynamik der Kopfgruppen spielt sich aber vermutlich auf einer sehr viel langsameren Zeitskala als der hier beobachteten ab und wird daher nicht zur Erklärung herangezogen [81]. Die genaue Ursache der langsamen Zeitkomponenten in eingeschränkten Umgebungen wurde von Nandi und Bagchi auf einen dynamischen Austausch zwischen gefangenen und gebundenen Wasserspezies zurückgeführt [98]. Das Auftauchen dieser langsamen Zeitkonstanten ist aber noch immer Gegenstand kontroverser Diskussionen und weit davon entfernt, im Detail verstanden zu sein.

Der starke Einfluß der Ladungen auf die Dynamik der Solvatation ist unter Umständen unmittelbar an einer Beteiligung der in Lösung befindlichen Gegenionen an der Solvatation gebunden, wie von Levinger und Mitarbeitern in inversen Mizellen gezeigt werden konnte [99, 100]. Eine andere Erklärung sind die unterschiedlichen Dicken der Stern'schen Doppelschicht in neutralen und geladenen Mizellen. In neutralen Mizellen (TX-100) wurde sie aus Kleinwinkel-Röntgenoder Neutronen-Streuexperimenten zu ca. $25 \AA$ bestimmt, während für ionische Mizellen (CTAB und SDS) eine Dicke von 6-9 9 ermittelt wurde. Dadurch ist in neutralen Mizellen der Farbstoff gänzlich von der reinen Wasserphase getrennt und weist eine entsprechend verlangsamte Dynamik auf, während in ionischen Zellen der Farbstoff zu einem gewissen Grad noch der reinen Wasserphase ausgesetzt ist und die Dynamik entsprechend weniger stark verlangsamt erscheint [81].

$\mathrm{Zu}$ beachten ist, daß die Zeitauflösung der hier vorgestellten Untersuchungen auf ca. 80 ps beschränkt ist, weswegen sich die Methode nicht zur Untersuchung der ultraschnellen Dynamik eignet. Der gezogene Vergleich zur Dynamik in reinem Wasser ist aufgrund der unterschiedlichen Zeitaufösungen daher nicht uneingeschränkt aussagekräftig, wie von Bhattacharyya auch angemerkt wurde [96]. Das Auftauchen extrem langsamer Zeitkomponenten ist nämlich nicht gleichbedeutend mit dem Verschwinden der ultraschnellen Zeitkomponenten. Mit diesen Methoden ist es daher nicht möglich zu entscheiden, ob die ultraschnelle Solvatation des Wassers an Mizellen noch relevant ist oder nicht.

Aufgrund dieser Beschränkungen durch die Zeitauflösung wurden die Experimente in jüngster Zeit von Bhattacharyya und Mitarbeitern mit Hilfe der Fluoreszenzkonversionsspektroskopie wiederholt [97]. Zur Verwendung kam der Farbstoff DCM in neutralen und kationischen Mizellen. Wie zuvor bereits beobachtet, erscheint die Solvatation an neutralen Mizellen deutlich langsamer als an ionischen Mizellen. Es zeigt sich aber zusätzlich, daß an diesen Grenzschichten tatsächlich 
ultraschnelle Zeitkomponenten mit erheblichem Anteil gefunden werden. So weist die neutrale Mizelle Zeitkonstanten von $2.1 \mathrm{ps}$ (30\%), $165 \mathrm{ps}$ (10\%) und $2050 \mathrm{ps}$ $(60 \%)$ auf und die kationische Mizelle Zeitkonstanten von 0.23 ps (50\%), 6.5 ps $(26 \%)$ und $350 \mathrm{ps}(24 \%)$.

Zusammenfassend gesehen wird deutlich, daß sich die Solvatationsdynamik an der Grenzschicht von Mizellen erheblich von der des reinen Wassers unterscheidet. Es tauchen langsame Zeitkonstanten im Nanosekundenbereich auf, die in reinem Wasser nicht beobachtet werden und deren Herkunft noch nicht eindeutig geklärt ist. Dennoch spielen bei der Solvatation an Mizellen auch ultraschnelle Komponenten weiterhin eine entscheidende Rolle, die vermutlich der Solvatation durch Wasser zuzuschreiben sind. Eine detaillierte Untersuchung dieser ultraschnellen Dynamiken an Mizellen blieb aber bislang aus.

\subsection{Inverse Mizellen}

Die ultraschnelle Solvatation von Wasser in inversen Mizellen ist Gegenstand der vorliegenden Arbeit. Um die erhaltenen Ergebnisse einordnen zu können, wird im folgenden versucht, die Fülle an Informationen, welche über inverse Mizellen existiert, zusammenzufassen. Einen Überblick bieten beispielsweise die Einführungen von Pileni [7] bzw. Luisi und Straub [8]. Eine Zusammenfassung neueren Datums bieten Moulik und Paul [101]. Bei der hier vorgestellten Zusammenstellung wird insbesondere Wert auf die Vorarbeiten bezüglich der Solvatationsdynamik gelegt, welche unter anderem in einem neueren Übersichtsartikel von Levinger behandelt werden [102].

\subsubsection{Struktur}

Als inverse Mizellen werden aus amphiphilen Molekülen bestehende Molekülaggregate bezeichnet, die sich in unpolaren Medien oberhalb einer kritischen Mizellenkonzentration (CMC, Critical Micelle Concentration) ausbilden können und sich dadurch auszeichnen, daß die polaren Kopfgruppen nach innen zeigen, während die unpolaren Kohlenwasserstoffketten nach außen in die unpolare Phase gerichtet sind. Es werden verschiedene amphiphile Moleküle als Mizellenbildner verwendet, wovon Aerosol-OT (AOT) [92, 100, 103, 104, 105, 106, 107], Triton X100 (TX-100) [108,109] und Lecithin [110,111] einige der gebräuchlichsten sind. Abbildung 3.2 zeigt ihre chemischen Strukturen.

Bei AOT handelt es sich um ein anionisches Molekül, welches den Vorteil besitzt ohne Hinzugabe sogenannter Hilfsdetergentien (Cosurfactants) inverse Mizellen zu formen. Darüberhinaus bildet es im Gegensatz zu vielen anderen Detergentien 


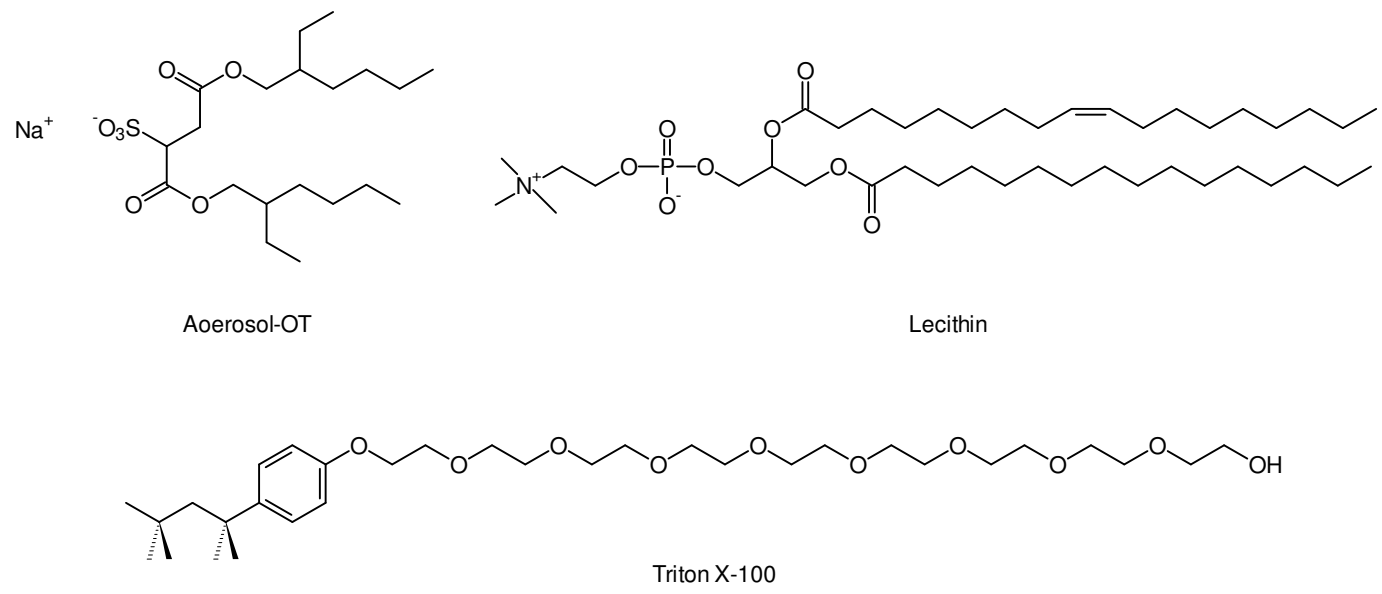

Abbildung 3.2: Chemische Strukturen einiger typischer Detergentien.

in Lösung annähernd kugelförmige Aggregate aus. Aus diesen Gründen ist es das zur Bildung inverser Mizellen am weitaus häufigsten eingesetzte Detergenz. Für die Ausbildung kationischer, inverser Mizellen sind in der Regel Hilfsdetergentien vonnöten, was ihre Herstellung erheblich erschwert. In jüngerer Zeit wurden auch vermehrt neutrale Detergentien wie z. B. Triton X-100 herangezogen. Es besitzt gegenüber AOT den Vorteil, daß es ungeladen ist und daher in Lösung befindliche Gegenionen nicht an der Solvatation in inversen Mizellen beitragen können. Wie von Levinger und Mitarbeitern gezeigt, hat die Natur des Gegenions nämlich erheblichen Einfluß auf die Solvatationsdynamik in inversen Mizellen [100], ähnlich wie bereits für die normalen Mizellen im vorangegangenen Kapitel beschrieben wurde.

Neben dem jeweiligen amphiphilen Molekül bestimmt auch die Wahl der organischen Phase die Struktur der sich formenden inversen Mizellen. Übliche verwendete Lösungsmittel sind z. B. Isooktan [105], $n$-Heptan [92, 104, 106, 107] und Cyclohexan [109, 111].

Eine herausragende Eigenschaft inverser Mizellen ist ihre Fähigkeit in ihrem Inneren polare Lösungsmittel aufzunehmen. Die resultierenden Systeme werden als Mikroemulsionen bezeichnet. Üblicherweise beinhalten die inversen Mizellen Wasser $[92,100,103,104,105,106,109,110,111]$, weshalb ihr Inneres auch als Wassereinschluß (Water Pool) oder Wassernanotröpfchen (Water-Nano-Droplet) bezeichnet wird. Im Falle des Systems Wasser/AOT können pro AOT-Molekül beispielsweise bis zu 50 Wassermoleküle innerhalb der inversen Mizelle gespeichert werden. Es wurden aber auch viele andere polare Lösungsmittel in inverse Mizellen eingebracht, wie z. B. Acetonitril [103,104,107,112,113], Alkohole [103,104,107,112,113] und Formamid [104,114], um deren Verhalten in eingeschränkten Umgebungen zu studieren. 
Der das Wassernanotröpfchen inverser Mizellen charakterisierende Parameter wird in der Literatur als $\mu$ oder $w_{0}$ bezeichnet und ist über das Verhältnis der Konzentration des polaren Lösungsmittels zur Konzentration des Detergenz gegeben:

$$
\mu=\frac{[\text { polares Lösungsmittel }]}{[\text { Detergenz }]}
$$

Dieser Parameter ist deshalb für die Charakterisierung inverser Mizellen von so großer Bedeutung, weil er in einem direkten Verhältnis zum Radius $r_{\mathrm{w}}$ des Nanotröpfchens steht. Für das in dieser Arbeit untersuchte System Wasser/AOT/Isooktan gilt beispielsweise $r_{\mathrm{w}} / \AA=1.5 \mu+4.5$ [115]. Aufgrund dieser einfachen Möglichkeit den Grad der Einschränkung über die Größe des Wassereinschlusses zu variieren, sind inverse Mizellen ein geeignetes Modellsystem für solche Umgebungen. Daraus erklärt sich auch die Popularität des Studiums inverser Mizellen, was eine Fülle an experimentellen Arbeiten nach sich zieht.

Die strukturellen Informationen über inverse Mizellen und Mikroemulsionen wurden aus einer Vielzahl unterschiedlichster Techniken erhalten, u. a. der dynamischen Licht-Streuung (DLS), der Kleinwinkel-Neutronenstreuung (SANS), der Röntgenstreuung, der THz-Spektroskopie [116], der IR- und RamanSpektroskopie [117, 118, 119], der NMR-Spektroskopie [115, 120, 121], der ESRSpektroskopie [120], der dynamischen Differentialkalorimetrie (DSC) [120], der Photonkorrelationsspektroskopie [122], der Fluoreszenzspektroskopie [123] und der dielektrischen Relaxation [124]. Die Fülle der Informationen ist überwältigend, weshalb an dieser Stelle nicht näher auf die weitreichenden Eigenschaften dieser Systeme eingegangen werden kann. Einen sehr guten Überblick bieten Moulik und Paul [101], während der Übersichtsartikel von Nandi et al. [81] eine Vielzahl weiterer Literaturstellen bietet.

Ähnlich wie bei den gewöhnlichen Mizellen werden in inversen Mizellen verschiedene Wasserformen unterschieden. So werden die zwischen den Kopfgruppen der Detergentien befindlichen Wassermoleküle als "gefangen“ (Trapped) bezeichnet. Sie sind gänzlich immobilisiert, was auch durch MD-Simulationen von Faeder und Ladanyi [125] gezeigt wurde. In der Nähe der Kopfgruppen ist das Wasserstoffbrückennetzwerk im Vergleich zu reinem Wasser noch immer erheblich gestört. In diesem Bereich weist das Wasser vornehmlich diffusive Bewegungen auf. Diese Wasserschicht wird als „gebunden“ (Bound) bezeichnet. Innerhalb weniger Wasserschichten werden die Eigenschaften des reinen Wassers erreicht. Diese Wasserphase, die mit zunehmender Mizellengröße immer dominanter wird, wird aufgrund ihrer Ähnlichkeit zu reinem Wasser als „frei“ (Free) bezeichnet. Die Verhältnisse sind in Abbildung 3.3 schematisch dargestellt. 


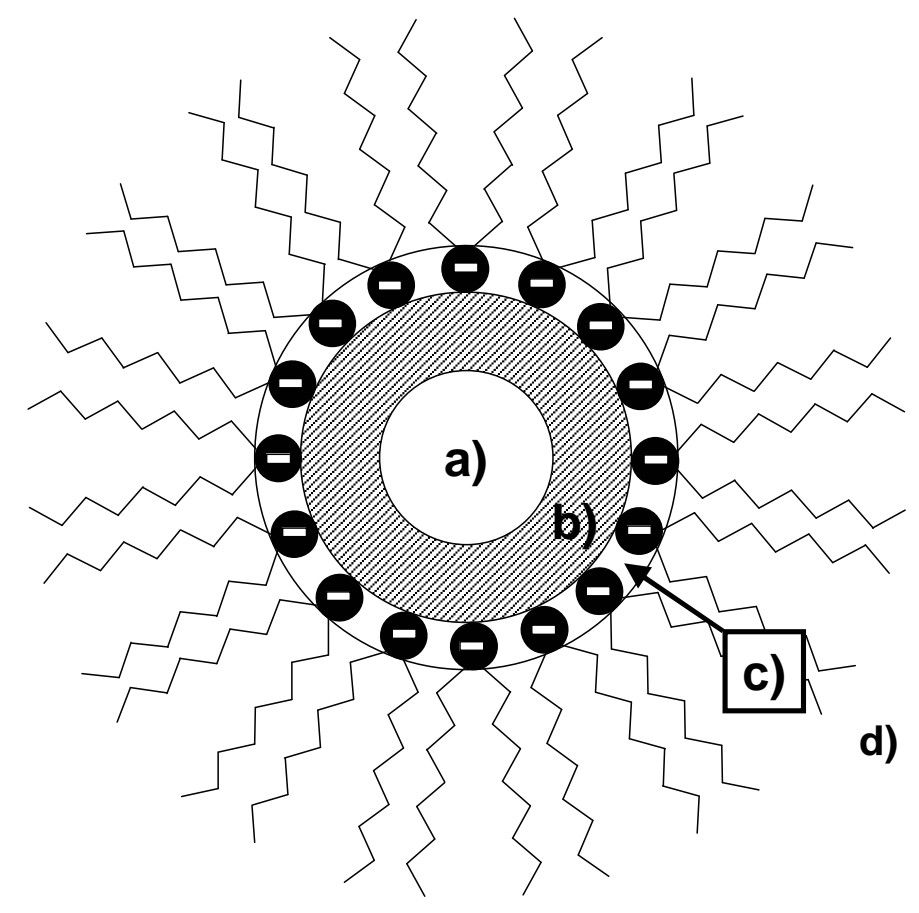

Abbildung 3.3: Schematischer Aufbau einer inversen Mizelle. a) Freies Wasser; b) Gebundenes Wasser; c) Gefangenes Wasser; d) Organische Phase.

\subsubsection{Solvatationsdynamik}

Die Solvatation in inversen Mizellen wurde von einer Reihe Arbeitsgruppen in den unterschiedlichsten Systemen untersucht. Dominiert wird die Literatur aber von den Arbeitsgruppen von Bhattacharyya [92,93], Levinger [105,126] und in neuerer Zeit Sarkar [103, 104,106]. Aufgrund der Vielzahl an Untersuchungen, sollen im folgenden nur Experimente an AOT/Wasser-Systemen vorgestellt werden, wie sie für die vorliegende Arbeit relevant sind.

Ein Problem bei allen durchgeführten Experimenten ist die eindeutige Kenntnis der Lage der verwendeten Farbstoffsonde [102, 127]. Bhattacharyya und Mitarbeiter zogen zur Klärung dieser Frage die stationären spektroskopischen Eigenschaften der Farbstoffe heran, während beispielsweise Levinger und Mitarbeiter zu diesem Zwecke Anisotropiemessungen durchführten [105]. Es zeigt sich bei all diesen Untersuchungen, daß sich der Chromophor in der Regel an der Grenzschicht des Wassereinschlusses anlagert. Ein anderer Ansatz stellt die gezielte Synthese neuer Farbstoffsonden dar, um die Position des Chromophors an der Grenzschicht gezielt festzulegen [127].

Bhattacharyya und Sarkar greifen bei ihren Untersuchungen auf die Technik des zeitkorrelierten Einphotonenzählens mit einer entsprechend begrenzten 
Zeitauflösung von etwas weniger als 100 Pikosekunden zurück. Repräsentativ für die Vielzahl an Experimenten sollen nachfolgend die Ergebnisse am System AOT/Wasser $/ n$-Heptan vorgestellt werden. In der Arbeitsgruppe von Bhattacharyya wurde es mit Hilfe der Farbstoffsonden Coumarin 480 [92] und DCM untersucht [93]. Sarkar vermaß das gleiche System mit den Farbstoffen Coumarin 490 [106], Coumarin 152 [104] und Coumarin 153 [103]. Die erhaltenen Solvatationskorrelationsfunktionen konnte in der Regel biexponentiell angepaßt werden. Die Ergebnisse sind in Tabelle 3.2 zusammengefaßt.

\begin{tabular}{|c|c|c|c|c|c|c|c|c|}
\hline Farbstoff & $\mu$ & $r_{\mathrm{w}} / \AA$ & $\tau_{1} / \mathrm{ns}$ & $A_{1}$ & $\tau_{2} / \mathrm{ns}$ & $A_{2}$ & $\langle\tau\rangle / \mathrm{ns}$ & Lit. \\
\hline C480 & $\begin{array}{c}4 \\
32\end{array}$ & $\begin{array}{c}8 \\
64\end{array}$ & $\begin{array}{c}8 \\
1.7\end{array}$ & $\begin{array}{c}1 \\
0.5\end{array}$ & $\begin{array}{c}- \\
12\end{array}$ & - & $\begin{array}{c}8 \\
6.85\end{array}$ & [92] \\
\hline DCM & 20 & 4 & 0.28 & 0.55 & 2.4 & 0.45 & 1.23 & [93] \\
\hline C490 & $\begin{array}{c}4 \\
32\end{array}$ & $\begin{array}{c}8 \\
64\end{array}$ & $\begin{array}{c}1.71 \\
0.668\end{array}$ & $\begin{array}{l}0.855 \\
0.956\end{array}$ & $\begin{array}{l}10.224 \\
11.084\end{array}$ & $\begin{array}{l}0.147 \\
0.048\end{array}$ & $\begin{array}{l}2.97 \\
1.17\end{array}$ & [106] \\
\hline C152 & 4 & 8 & 1.146 & 0.174 & 14.73 & 0.816 & 7.27 & [104] \\
\hline C153 & $\begin{array}{c}4 \\
32\end{array}$ & $\begin{array}{c}8 \\
64\end{array}$ & $\begin{array}{c}8.4 \\
0.933\end{array}$ & $\begin{array}{c}1 \\
0.48\end{array}$ & $\begin{array}{c}- \\
10.24\end{array}$ & $\begin{array}{c}- \\
0.52\end{array}$ & $\begin{array}{c}8.4 \\
5.77\end{array}$ & [103] \\
\hline
\end{tabular}

Tabelle 3.2: Abklingverhalten der Solvatationskorrelationsfunktion im System Wasser/AOT/n-Heptan bestimmt durch zeitkorreliertes Einzelphotonenzählen.

Es zeigt sich, daß bei der Solvatation in inversen Mizellen langsame Zeitkonstanten eine Rolle spielen, die bei der Solvatation in reinem Wasser nicht beobachtet werden. Die Dynamik scheint durch die eingeschränkte Umgebung signifikant gehindert und entsprechend verlangsamt zu sein. Beim Übergang von kleineren zu größeren inversen Mizellen werden die beobachteten Zeiten aber insgesamt deutlich schneller. Dennoch bleibt auch in den größten untersuchten Mizellen eine deutlich verlangsamte Zeitkonstante $\tau_{2}$ bestehen, d. h. die beobachtete Dynamik ist im Vergleich zu reinem Wasser noch immer bemerkenswert verlangsamt. Interessant ist dabei, daß diese Komponente $\tau_{2}$ deutlich langsamer ist als bei den normalen Mizellen (vgl. Tabelle 3.1). Aufgrund der geringen Zeitauflösung des zeitkorrelierten Einzelphotonenzählens ist es aber auch hier nicht zu entscheiden, ob an der Solvatation weiterhin ultraschnelle Komponenten teilhaben. Eine Abschätzung von Hazra et al. deutet jedoch bereits darauf hin, daß mit dieser Technik lediglich 20-40\% des gesamten Stokes-Shifts erfaßt werden [103]. 
Levinger und Mitarbeiter untersuchten daher die ultraschnelle Solvatationsdynamik im System Wasser/AOT/Isooktan mit Hilfe der Fluoreszenzaufkonvertierung und dem Farbstoff Coumarin 343. Sie erreichen in ihren Experimenten eine Zeitauflösung um $180 \mathrm{fs}$, was ihnen einen deutlich größeren Dynamikbereich eröffnet und ausreichen sollte, um zu entscheiden, ob ultraschnelle Solvatation eine Rolle spielt. Die erhaltenen Solvatationskorrelationsfunktionen wurden biexponentiell angepaßt und sind in Tabelle 3.3 aufgeführt. Zum Vergleich wurden die Experimente sowohl in reinem Wasser als auch in einer einmolaren Natriumsalz-Lösung durchgeführt, die den tatsächlichen Verhältnissen in den ionischen Wassernanotröpfchen eher entsprechen sollte.

\begin{tabular}{|c|c|c|c|c|c|}
\hline $\boldsymbol{\mu}$ & $\boldsymbol{\tau}_{\mathbf{1}} / \mathbf{p s}$ & $\boldsymbol{A}_{\mathbf{1}}$ & $\boldsymbol{\tau}_{\mathbf{2}} / \mathbf{p s}$ & $\boldsymbol{A}_{\mathbf{2}}$ & $\langle\boldsymbol{\tau}\rangle / \mathrm{ps}$ \\
\hline 5 & 0.25 & 0.43 & 3.3 & 0.57 & 1.99 \\
7.5 & 0.11 & 0.69 & 3.0 & 0.31 & 1.01 \\
15 & 0.24 & 0.68 & 2.9 & 0.34 & 1.15 \\
40 & 0.16 & 0.57 & 2.2 & 0.43 & 1.04 \\
\hline $\begin{array}{c}1 \mathrm{M} \mathrm{Na} \\
\text { reines Wasser }\end{array}$ & 0.21 & 0.54 & 1.3 & 0.46 & 0.72 \\
\hline
\end{tabular}

Tabelle 3.3: Abklingverhalten der Solvatationskorrelationsfunktion im System Wasser/AOT/Isooktan bestimmt durch Fluoreszenzaufkonvertierung mit der Farbstoffsonde Coumarin 343 [105].

Es zeigt sich, daß in der Tat sehr schnelle Zeitkomponenten gefunden werden, die denen des reinen Wassers ähneln. Die schnellere Zeitkomponente um $0.2 \mathrm{ps}$ wird in allen untersuchten Proben gefunden und wurde der Solvatation im Inneren des Wassernanotröpfchens der Mizellen zugesprochen, da sie der in reinem Wasser beobachteten Zeitkonstanten entspricht. Die langsamere Komponente wurde dem gebundenen Wasser zugeschrieben. Diese Zeitkonstante wird mit wachsender Zellengröße immer schneller, was mit dem kleiner werdendem Anteil an gebundenem Wasser erklärt wird. Zudem wurde in allen Proben eine langsame Zeitkonstante zwischen 200 und 400 ps gefunden, die umso schneller wird, je größer die Mizellen werden (in Tabelle 3.3 nicht aufgeführt). Sie erreicht aber niemals den Wert des freien Wassers und wird daher kollektiven Moden des Wassers an der Grenzfläche zugeschrieben. Interessant ist auch der Unterschied zwischen reinem Wasser und der einmolaren Natriumsalz-Lösung. Die Ionen haben offensichtlich einen deutlich verlangsamenden Einfluß auf die beobachtete Wasserdynamik. Dieser Effekt ist abhängig von der Natur des Kations, wie durch Variation des Gegenions des AOT's an den Beispielen $\mathrm{Na}^{+}$und $\mathrm{NH}_{4}^{+}$[99] bzw. $\mathrm{K}^{+}$und $\mathrm{Ca}^{2+}$ [100] gezeigt werden konnte. In einer erneuten Analyse der Daten mit Hilfe der Me- 
thode nach Fee und Maroncelli [26], zeigte sich aber, daß auch mit der hier vorgestellten Methode nur ein geringer Anteil der Solvatationskorrelationsfunktion aufgelöst werden konnte [102].

Die Hauptaussage der bisherigen Untersuchungen zur ultraschnellen Solvatation in inversen Mizellen ist, daß die Solvatationsdynamik ähnlich wie bei den normalen Mizellen insgesamt gesehen stark verlangsamt erscheint. Es werden Zeitkomponenten gefunden, die in reinem Wasser nicht beobachtet werden. Dennoch haben ultraschnelle Zeitkomponenten noch immer eine entscheidenden Anteil bei der Solvatation in den Wassernanotröpfchen inverser Mizellen. Eine gänzlich befriedigende Untersuchung der Dynamik auf ultraschnellen Zeitskalen blieb bislang aber aus.

\subsubsection{MD-Simulationen}

In den letzten Jahrzehnten wurden nur wenige molekulardynamische Simulationen durchgeführt, um die Verhältnisse in inversen Mizellen besser verstehen zu können [128, 129, 130]. Erst kürzlich wurden Arbeiten von Faeder und Ladanyi veröffentlicht, deren Resultate sich direkt mit experimentellen Daten vergleichen lassen $[125,131]$. Die erste Arbeit befaßt sich mit der Struktur des Mizelleninneren und belegt deutlich, daß die oben vorgestellte Unterteilung in drei deutlich verschiedene Bereiche innerhalb des Wassernanotröpfchens gerechtfertigt ist [125]. In Konsistenz mit den experimentellen Resultaten finden sie, daß die Beweglichkeit des Wassers in Nähe der Grenzfläche stark gehindert ist. Die Eigenschaften des reinen Wassers werden aber innerhalb weniger Wasserschichten erreicht. Die zweite Arbeit simuliert die Solvatationsdynamik in unterschiedlich großen inversen Mizellen, wobei ein Zeitfenster bis 2 ps betrachtet wird [131]. Es zeigt sich in grober Übereinstimmung mit den experimentellen Erkenntnissen, daß die Solvatation in diesem Zeitfenster mit wachsender Mizellengröße schneller wird, auch wenn der Haupteffekt im langsameren, diffusiven Anteil stattfindet. Der beobachtete Inertialteil, der unabhängig von der Mizellengröße ca. $70 \%$ der beobachteten Solvatationsantwort ausmacht, erscheint jedoch weitestgehend unabhängig vom Grad der Einschränkung. 


\subsection{Lipid-Vesikel}

In diesem Abschnitt soll eine kurze Einführung in das Gebiet der LipidmembranVesikel und deren Bestandteile, den Phospholipiden, gegeben werden. Da Membranen einer der Hauptbestandteile von Zellen und Zellorganellen sind, ist die Informationsfülle auf diesem Gebiet entsprechend groß. Für den in dieser Arbeit betrachteten Themenkomplex sind die Standardwerke von Cevc und Marsh [4], Grell [5] und Shinitzky [6] zu empfehlen. Überraschenderweise sind die Studien zur Solvatation an Lipidmembran/Wasser-Grenzschichten allerdings deutlich weniger zahlreich als im Falle der inversen Mizellen. Die Literatur auf diesem Gebiet resultiert vorrangig aus den Gruppen von Bhattacharyya [89, 90, 91], Hof [132, 133, 134, 135, 136, 137, 138, 139] und Parasassi [140, 141, 142]. Die ultraschnelle Solvatation wurde in jüngster Zeit in der Arbeitsgruppe von Vöhringer mit Hilfe der Photon-Echo Peakshift Technik untersucht $[27,50,79,143]$.

\subsubsection{Phospholipide}

Lipide sind Musterbeispiele amphiphiler Moleküle und besitzen in der Natur ein extrem breites Spektrum an biologischen Funktionen [9,43]. Sie dienen u. a. als Energiespeicher, als Signalmoleküle und vorrangig als Hauptbestandteile biologischer Membranen. In Membranen sind drei Hauptgruppen von Lipiden anzutreffen: Phospholipide, Glykolipide und Cholesterin. Phospholipide stellen dabei den Hauptbestandteil dar und sind Thema der vorliegenden Arbeit. Die Phospholipide leiten sich entweder vom Glycerin oder vom Sphingosin ab, weswegen sie entsprechend als Glycerophospholipide bzw. Phosphoglyceride oder Sphingophospholipide bezeichnet werden. Die Glycerophospholipide setzen sich aus einem Glycerinrückgrat, zwei Fettsäureketten und einem phosphorylierten Alkohol zusammen. Je nach Art des Alkohols wird zwischen Phosphatidylcholinen, Phosphatidylethanolaminen, Phosphatidylglycerolen, Phosphatidylinositolen und Phosphatidylserinen unterschieden. Die beiden Fettsäureketten können gleich (symmetrisch), aber auch unterschiedlich (asymmetrisch) sein. Sie enthalten in der Regel eine gerade Anzahl von Kohlenstoffatomen, meist zwischen 14 und 24. Die Kohlenwasserstoffketten der Fettsäuren sind zumindest in tierischen Organismen unverzweigt, können aber gesättigt oder ungesättigt sein, wobei es sich im letzteren Falle meist um cis-Konfigurationen handelt. Desweiteren unterscheiden Phospholipide sich in ihrer jeweiligen absoluten Konfiguration, weshalb verschiedene Stereoisomere unterschieden werden können. Die Vielfalt unterschiedlicher Lipide ist aufgrund dieser Variationsmöglichkeiten sehr groß. All diese Faktoren beeinflussen dabei die Struktur und Eigenschaften einer Membran erheblich und müssen entsprechend beachtet werden. Für eine tiefergehende Einführung sei auf Lehrbücher der Biochemie verwiesen [9, 43]. 
Bei den in diesem Rahmen dargestellten Untersuchungen zu Solvatationsdynamik kamen die folgenden Phospholipide zum Einsatz:

- DMPC: 1,2-Dimyristoyl-sn-glycero-3-phosphatidylcholin (14:14)

- DPPC: 1,2-Dipalmitoyl-sn-glycero-3-phosphatidylcholin (16:16)

- DHPC: 1,2-Dihexadecyl-sn-glycero-3-phosphatidylcholin (16:16)

Dabei handelt es sich um gesättigte, symmetrische Phosphatidylcholine mit zwei Alkylketten bestehend aus jeweils 14 (DMPC) bzw. 16 (DPPC und DHPC) Kohlenstoffatomen. Beim DHPC handelt es sich um eine Variante von DPPC, bei der die Carbonylgruppen reduziert sind, so daß es sich im Gegensatz zu einem Ester zwischen Fettsäureresten und Glycerin um einen Ether handelt.

\subsubsection{Vesikel}

Phospholipide haben aufgrund ihres amphiphilen Charakters und des hydrophoben Effektes die Eigenschaft in Wasser Molekülaggregate zu bilden. Diese Selbstorganisation führt zu erstaunlichen Strukturen, so bilden sich u. a. hexagonale, kubische und lamellare Phasen aus. Da Übergänge zwischen diesen Phasen durch die Änderung des Wasser/Lipid-Verhältnisses eingeleitet werden können, wird das Verhalten als lyotroper Polymorphismus bezeichnet. Die häufigsten Strukturen stellen dabei die lamellaren Phasen dar. In Lösung bilden sich zunächst gestapelte Lipid-Doppelschichten (Lipid-Bilayers), die durch eine dünne Wasserschicht getrennt sind. Mit zunehmendem Wassergehalt wird diese Schicht immer dicker, was schließlich dazu führt, daß die Strukturen zu Vesikeln zerfallen. Die auch als Liposome bezeichneten Vesikel werden nach Größe und Beschaffenheit unterschieden. So wird zunächst zwischen unilamellaren und multilamellaren Vesikeln (MLV, Multilamellar Vesicles) eingeteilt. Die besonders interessanten unilamellaren Vesikel werden in sogenannte kleine unilamellare Vesikel (SUV, Small Unilamellar Vesicles), große unilamellare Vesikel (LUV, Large Unilamellar Vesicles) und riesige Vesikel (GUV, Giant Unilamellar Vesicles) unterteilt. Ihre Durchmesser reichen von kleiner $100 \mathrm{~nm}$ bei den SUV's bis in den Bereich von $20-100 \mu \mathrm{m}$ bei den Riesenvesikeln. Damit ist der Wassereinschluß von Vesikeln deutlich größer als im Falle der inversen Mizellen $\left(r_{\mathrm{w}}<10 \mathrm{~nm}\right)$. Um gezielt Vesikel einer bestimmten Größe herstellen zu können, wurden verschiedene Methoden entwickelt. Während Riesenvesikel sich durch Elektroformationsmethoden herstellen lassen, ist die übliche Methode für die Herstellung von SUV's die Behandlung multilamellarer Strukturen im Ultraschallbad. Die bei dieser Methode entstehenden Strukturen zeigen jedoch breite Größenverteilungen. Mit der Methode des Extrudierens lassen sich schmalere Verteilungen der SUV's erhalten. 
Dabei werden die multilamellaren Strukturen durch Mikrofilter definierter Porengröße gepreßt. Unilamellare Vesikel stellen die einfachsten Modelle biologischer Membranen dar und eignen sich daher hervorragend zum systematischen Studium der Eigenschaften natürlicher Membranen.

\subsubsection{Polymorphismus}

Eine bemerkenswerte Eigenschaft von Lipidmembranen ist ihr Phasenverhalten [144]. In Abhängigkeit der Temperatur bilden sich strukturell unterschiedliche Phasen aus, weshalb dieser Effekt als thermotroper Polymorphismus oder einfach Polymorphismus bezeichnet wird. Es existieren eine Reihe verschiedener Lipidphasen. Sie werden mit Großbuchstaben L für ein eindimensionales lamellares Gitter und P für ein zweidimensionales zentriertes Gitter bezeichnet. Der Index $\alpha$ kennzeichnet fluide Phasen, während $\beta$ kristalline Strukturen bezeichnet. Ein Apostroph steht für bezüglich der Membrannormalen abgewinkelte Lipidmoleküle. Für die Untersuchung von Phasenübergängen ist die Differentialkalorimetrie (DSC) die Methode der Wahl (siehe Kapitel 4.1). Im folgenden sollen nur die sogenannten Hochtemperaturphasen $\mathrm{L}_{\beta}, \mathrm{P}_{\beta}$, und $\mathrm{L}_{\alpha}$ betrachtet werden, deren Strukturen schematisch in Abbildung 3.4 dargestellt sind. Sie werden als gelförmige Phase $\left(\mathrm{L}_{\beta}\right)$, Rippled-Phase $\left(\mathrm{P}_{\beta},\right)$ und flüssig-kristalline Phase $\left(\mathrm{L}_{\alpha}\right)$ bezeichnet. Ihre unterschiedlichen Strukturen werden im folgenden kurz behandelt.

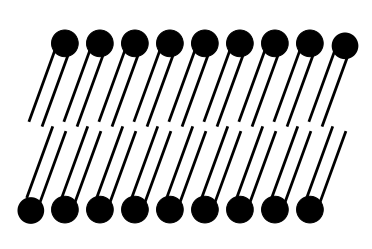

$\mathrm{L}_{\beta^{\prime}}$

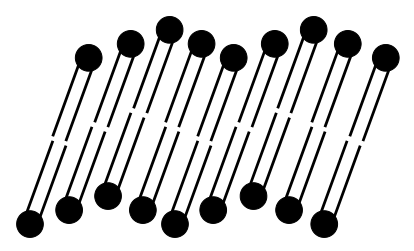

$\mathrm{P}_{\beta^{\prime}}$

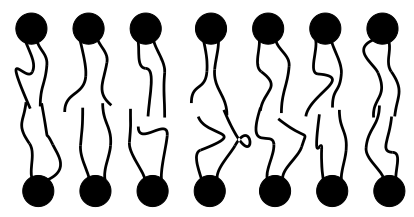

$\mathrm{L}_{\alpha}$

Abbildung 3.4: Unterschiedliche Phasenzustände in Lipidmembranen. Links: Gelförmige Phase $\left(L_{\beta},\right)$; Mitte: Rippled-Phase $\left(P_{\beta},\right)$; Rechts: Flüssig-kristalline Phase $\left(L_{\alpha}\right)$.

\section{Gelförmige Phase $\left(\mathrm{L}_{\beta},\right)$}

In der gelförmigen Phase liegen die Fettsäureketten der Phospholipide in einer all-trans-Konformation vor. Sie stellt für die meisten Phospholipide die erste der Hochtemperaturphasen dar, während andere beispielsweise eine kristalline $\mathrm{L}_{\beta}$-Phase bevorzugen. In der gelförmigen Phase sind die Ketten um ca. $30^{\circ}$ gegenüber der Membrannormalen geneigt. Sie zeichnet sich gegenüber den kristallinen 
Niedertemperaturphasen durch eine Rotationsunordnung aus, d. h. die Lipidketten sind in Bezug auf ihre Orientierung zueinander nicht vollständig geordnet. Die Übergänge zwischen den einzelnen Phasen sind zweiter Ordnung. Der Übergang von der $\mathrm{L}_{\beta}$,- in die $\mathrm{P}_{\beta}$,Phase wird allerdings nur in Lipiden mit relativ großen Kopfgruppen beobachtet, wie z. B. den Phosphatidylcholinen. Je länger die Ketten werden, desto näher rücken die Temperaturen der beiden Phasenübergänge zusammen. So wird bei gesättigten Phosphatidylcholinen ab einer Kettenlänge von 24 Kohlenstoffatomen beispielsweise ein direkter Übergang von der gelförmigen in die flüssig-kristalline Phase beobachtet, ohne daß eine Rippled-Phase durchlaufen wird. Ebenso wird ein direkter Übergang in die flüssig-kristalline Phase beobachtet, wenn der Wassergehalt weniger als ca. 20 Gewichtsprozente beträgt. Der $\left(\mathrm{L}_{\beta}, \rightarrow \mathrm{P}_{\beta}\right.$ ) - Übergang, der auch als Vorphasenübergang bezeichnet wird, ist vorrangig von einer Neuordnung der Lipidkopfgruppen und nur in einem sehr geringen Maße von einer Neuordnung der Ketten bestimmt. Dies zeigt sich beispielsweise darin, daß die Umwandlungsenthalphie am Vorphasenübergang unabhängig von der Kettenlänge der Lipide ist. Bei dieser Neuordnung nimmt allerdings die Hydratisierung der Kopfgruppen entscheidend zu, was mit einer Reihe von Techniken bestätigt werden konnte [145, 146, 147].

\section{Rippled-Phase $\left(\mathbf{P}_{\beta},\right)$}

Die Rippled-Phase besitzt eine charakteristische Überstruktur, welche sich als zackenartiges Oberflächenprofil darstellt. Diese Struktur wurde erstmals mit Hilfe der Röntgenstrukturanalyse an multilamellaren Schichten beobachtet. Die Zacken bilden sich in Abhängigkeit des Wassergehaltes aus und weisen in Phosphatidylcholinen räumliche Perioden zwischen $145 \AA$ und $164 \AA$ auf. Der als Hauptphasenübergang bezeichnete $\left(\mathrm{P}_{\beta}, \rightarrow \mathrm{L}_{\alpha}\right)$ - Übergang (bzw. der $\left(\mathrm{L}_{\beta}, \rightarrow \mathrm{L}_{\alpha}\right)$ - Übergang bei fehlender Rippled-Phase) geht mit dem Schmelzen der Kettenbereiche einher. Dabei gehen die Kohlenwasserstoffketten von den gestreckten all-trans-Zuständen in eine ungeordnete, knäulartige Konformation über. Daher besteht hier eine Analogie zum bekannten Glasübergang in Polymeren.

\section{Flüssig-kristalline Phase $\left(\mathrm{L}_{\alpha}\right)$}

Die flüssig-kristalline Phase ist durch die knäulförmige Konfiguration ihrer Ketten gekennzeichnet. Ihre Ordnung entspricht eher der einer Flüssigkeit als der eines Festkörpers. Die benötigte Fläche pro Lipidmoleküle ist um ca. 30\% größer als in der gelförmigen Phase. Sie stellt die physiologisch relevanteste Phase dar und ist entsprechend die am intensivsten untersuchte Membranphase. In ihr kann Wasser recht tief in die hydrophobe Lipidphase eindringen, was durch MD-Simulationen und Röntgenbeugungsanalysen belegt werden konnte. 


\subsubsection{Solvatationsdynamik}

Das Studium der Solvatationsdynamik mit Hilfe des zeitaufgelösten Stokes-Shifts in Lipidvesikeln wird vorrangig von den Arbeiten von Hof und Mitarbeitern dominiert $[132,133,134,135,136,137,138,139]$. Sie verwenden dabei die Technik des zeitkorrelierten Einzelphotonenzählens mit entsprechend begrenzter Zeitauflösung, wobei vorrangig die Farbstoffsonden Prodan und Patman eingesetzt wurden. Sie führten ihre Experimente an einer Reihe unterschiedlichster Systeme durch. Beispielhaft sind in Tabelle 3.4 einige ihrer Ergebnisse an Membranen bestehend aus DMPC und DHPC zusammengestellt. Da die Ergebnisse nicht biexponentiell angepaßt werden konnten, wird hier ein Vergleich über eine mittlere Solvatationszeit angestellt, die definiert ist als:

$$
\langle\tau\rangle=\int_{0}^{\infty} S(t) \mathrm{d} t
$$

Dabei sind beide Definitionen der mittleren Solvatationszeit, d. h. die Gleichungen 3.1 und 3.3, gleichwertig. Gleichung 3.3 ist dabei eine allgemeine Definition, die im Spezialfall für biexponentiell anpaßbare Solvatationskorrelationsfunktionen in Gleichung 3.1 übergeht.

\begin{tabular}{|c|c|c|c|c|c|}
\hline Lipid & Farbstoff & Phase & $\boldsymbol{T} /{ }^{\circ} \mathbf{C}$ & $\langle\boldsymbol{\tau}\rangle / \mathbf{n s}$ & Lit. \\
\hline \multirow{4}{*}{ DMPC } & \multirow{2}{*}{ Prodan } & & 30 & 3.5 & \\
& & $\mathrm{~L}_{\alpha}$ & 43 & 1.8 & \\
& \multirow{2}{*}{ Patman } & & 43 & 0.7 & \\
& & & 2.2 & {$[134]$} \\
\hline \multirow{3}{*}{ DHPC } & \multirow{2}{*}{ Patman } & $\mathrm{P}_{\beta}$, & 20 & 2.6 & \\
& & $\mathrm{P}_{\beta}$, & 40 & 2.0 & {$[135]$} \\
& & $\mathrm{L}_{\alpha}$ & 52 & 0.7 & \\
\hline
\end{tabular}

Tabelle 3.4: Mittlere Solvatationszeit $\langle\tau\rangle$ in den Lipidmembranen DMPC und DHPC in Abhängigkeit der Temperatur und der Farbstoffsonde bestimmt durch zeitkorreliertes Einzelphotonenzählen.

Die Ergebnisse zeigen, daß die mittlere Zeitkonstante $\langle\tau\rangle$ mit steigender Temperatur immer schneller wird, was durch die erhöhte Beweglichkeit der Membranen interpretiert werden kann. Die beobachteten Zeitkonstanten sind dabei erwartungsgemäß von der jeweiligen Natur des untersuchten Lipids abhängig. Dabei ist beim Überschreiten des Hauptphasenübergangs ein größerer Sprung zu beobachten, wie an DPPC-Vesikeln mit dem Farbstoff TMA eindrücklich gezeigt werden konnte [132]. Auch diese Beobachtung ist mit der starken Erhöhung der 
Membranflexibilität beim Übergang in die flüssig-kristalline Phase zu verstehen. Desweiteren sind die Zeitkonstanten auch vom verwendeten Farbstoff abhängig.

In Lipidmembranen ändert sich die Dielektrizitätskonstante innerhalb eines Raumabschnittes von weniger als drei Nanometern von ca. 2 im Inneren der Kettenregion bis ca. 80 direkt über den Kopfgruppen, wobei der letztere Wert für die freie Wasserphase charakteristisch ist. Diese starke Variation der Polarität entlang der Membrannormalen macht deutlich, daß es von grundlegender Bedeutung ist, die genaue Lokalisierung der verwendeten Farbstoffsonde in der Membran zu kennen, da die in solchen Umgebungen beobachte Solvatationsdynamik somit auch eine Funktion des Ortes ist. Die Veränderung der beobachteten Dynamiken in Abhängigkeit der Einbautiefe wurde eindrücklich mit den Farbstoffsonden $n$-AS gezeigt, wobei $n=2,3,6,9,12,16$ ist und in Beziehung zur Position des Chromophors innerhalb der Membran steht [136]. Es zeigt sich, daß mit tieferer Verankerung des Chromophors in der Membran, ein kleinerer Stokes-Shift und immer langsamere mittlere Solvatationszeiten auftreten. Leider sind diese Untersuchungen nicht für unterschiedliche Phasenzustände von Membranen bekannt. In einer kürzlich erschienenen Arbeit von Hof und Mitarbeitern wurden sieben verschiedene Farbstoffe in der Membran gegenübergestellt und gezeigt, daß sie alle verschiedene Solvatationszeiten aufweisen [139]. Die Unterschiede wurden dabei mit den unterschiedlich tiefen Einbaulagen der Farbstoffe in der Membran begründet. So scheinen sich einige Chromophore tief im hydrophoben Kern anzulagern, während andere sich relativ weit in der Wasserphase befinden. Eine weitere interessante Untersuchung ist der Vergleich von Relaxationszeiten in SUV's $\left(r_{\mathrm{w}}=11 \mathrm{~nm}\right)$ und LUV's $\left(r_{\mathrm{w}}=125 \mathrm{~nm}\right)$. Es zeigte sich, daß für die Farbstoffsonde TNS, welche sich an der Grenzschicht der Vesikel anlagert, die Relaxationszeit in SUV's deutlich schneller ist als in LUV's. Für die Probe 12-AS, die sich im hydrophoben Kern befindet, wurde praktisch keine Abhängigkeit gefunden. Dieses Verhalten wurde mit der starken Krümmung der Membran in den SUV's erklärt, was dazu führt, daß die Kopfgruppen weniger dicht gepackt sind und es daher zu einer höheren Beweglichkeit in der Kopfgruppenregion in solchen Systemen kommt. Die Solvatation an der Grenzschicht erweist sich als entsprechend beschleunigt.

Von Bhattacharyya und Mitarbeitern wurde die Solvatation an SUV's der Phosphatidylcholine DMPC und DPPC mit Hilfe der Farbstoffe DCM und Coumarin 480 mit der gleichen Technik untersucht. Im Gegensatz zu Hof und Mitarbeitern wurden ihre Solvatationskorrelationsfunktionen biexponentiell angepaßt. Die erhaltenen Parameter zeigt Tabelle 3.5. Wie in ihren vorherigen Studien an Mizellen und inversen Mizellen finden Bhattacharyya und Mitarbeiter zwei Zeitkonstanten, wovon die erste im Bereich zwischen 100 und 600 ps liegt und die zweite auf einer Zeitskala von 1 bis 10 ns. Diese Zeiten ähneln den in größeren inversen Mizellen beobachteten Zeitkonstanten. Die langsamere der beiden wird daher analog dem an der Grenzfläche gebundenem Wasser zugeordnet und die schnellere den gehinderten Bewegungen von Wassermolekülen im inneren Wassereinschluß 


\begin{tabular}{|c|c|c|c|c|c|c|c|c|}
\hline Lipid & Farbstoff & Phase & $\boldsymbol{T} /{ }^{\circ} \mathbf{C}$ & $\boldsymbol{\tau}_{\mathbf{1}} / \mathbf{n s}$ & $\boldsymbol{A}_{\mathbf{1}}$ & $\boldsymbol{\tau}_{\mathbf{2}} / \mathbf{n s}$ & $\boldsymbol{A}_{\mathbf{2}}$ & Lit. \\
\hline DMPC & $\mathrm{C} 480$ & $\mathrm{~L}_{\alpha}$ & 26 & 0.60 & 0.4 & 11 & 0.6 & {$[89]$} \\
DMPC & DCM & $\mathrm{L}_{\alpha}$ & 26 & 0.23 & 0.4 & 1.6 & 0.6 & {$[90]$} \\
DPPC & DCM & $\mathrm{L}_{\beta}$ & 20 & 0.12 & 0.2 & 5.5 & 0.8 & {$[91]$} \\
\hline
\end{tabular}

Tabelle 3.5: Abklingverhalten der Solvatationskorrelationsfunktion in den Lipidmembranen DMPC und DPPC in Abhängigkeit der Temperatur und der Farbstoffsonde bestimmt durch zeitkorreliertes Einzelphotonenzählen.

der Vesikel. In all diesen Arbeiten können ultraschnelle Zeitkomponenten aufgrund der limitierten Zeitauflösung nicht erfaßt werden.

Von Vöhringer und Mitarbeitern wurde in jüngster Zeit zur Klärung dieser bisher nicht beantworteten Fragestellung die 3-Puls Photon-Echo Peakshift Technik auf unilamellare Vesikel angewendet $[27,50,79,143]$. Dabei kam die Farbstoffsonde C18-DiD zum Einsatz, wobei es sich um ein Derivat des Farbstoffes HIDCI handelt. Der Farbstoff ist durch seine langen Alkylketten nicht-kovalent in der Membran verankert, wodurch der Chromophor eine genau definierte Position unmittelbar über den Kopfgruppen der Lipide besitzt. In den durchgeführten Arbeiten wurde unter anderem die Solvatation an DMPC- und DPPC-Lipidmembranen in Abhängigkeit ihres Phasenzustandes untersucht. Dabei zeigte sich, daß bei der Solvatation an Lipidmembran/Wasser-Grenzflächen ultraschnelle Zeitkomponenten weiterhin dominieren. Ähnlich wie in der reinen Wasserphase, finden sich hierbei in allen Messungen ausgeprägte Inertialteile wieder. Ebenso wird in allen Systemen und allen Phasenzuständen eine Zeitkonstante um 10 ps gefunden. Wie in Kapitel 3.1 beschrieben, wird diese den diffusiven Reorientierungen einzelner Wassermoleküle zugeschrieben. Die ausgeprägte Zeitkomponente um 1 ps, welche die Solvatationsdynamik in reinem Wasser entscheidend bestimmt, zeigt jedoch ein unterschiedliches Verhalten in Abhängigkeit des untersuchten Phasenzustandes. Oberhalb des Vorphasenübergangs erscheint diese Mode vollkommen unterdrückt, während sie in der gelförmigen Phase stark an der Solvatation beteiligt ist. Beim Übergang von der Rippled-Phase in die flüssig-kristalline Phase wird keine Änderung der Solvatationsdynamik beobachtet. Offensichtlich ist das Wasserstoffbrückennetzwerk in der Rippled- und flüssig-kristallinen Phase stark gestört, während es in der gelförmigen Phase weitestgehend den Charakter des reinen Wassers beibehält. Diese Ergebnisse sind im Einklang mit der oben getroffenen Aussage, daß der Vorphasenübergang vorrangig mit einer Umstrukturierung in der Kopfgruppenregion einhergeht, was unmittelbaren Einfluß auf das Wasserstoffbrückennetzwerk und damit verbunden der beobachteten Solvatationsdynamik hat. Entsprechend ist am Hauptphasenübergang auf ultraschnellen Zeitskalen kein Effekt zu beobachten, da dieser Übergang vor allem durch das Schmelzen der Kohlen- 
wasserstoffketten in der Membran charakterisiert ist. Aufgrund seiner spezifischen Einbaulage sind die spektroskopischen Eigenschaften des verwendeten Chromophors hierauf nicht empfindlich. Darüberhinaus werden in allen Untersuchungen langsame Zeitkomponenten im Bereich einiger hundert Pikosekunden gefunden, die keine Abhängigkeit vom jeweiligen Phasenzustand zeigen. Diese Komponente ist nach Vöhringer und Mitarbeitern vermutlich der kollektiven Rotationsdiffusion des Wassers und/oder der Rotationsdiffusion des verwendeten Chromophors zuzuordnen.

\subsubsection{MD-Simulationen}

In der Literatur existiert eine Vielzahl molekulardynamischer Simulationen von Lipidmembran/Wasser-Grenzschichten [148,149,150,151,152,153,154,155]. Schulten und Mitarbeiter konnten dabei die unterschiedlichen Strukturen der kristallinen, der gelförmigen und der flüssig-kristallinen Phase in Lipid-Doppelschichten nachweisen [148]. So zeigen diese Simulationen sehr anschaulich die sich deutlich ändernden Strukturen beim Übergang in die jeweils nächsthöhere Phase. Außerdem liefern solche Simulationen Informationen bezüglich der Polarisation des Wassers im Bereich der Grenzschicht und über dessen Eindringtiefe in den hydrophoben Kern der Membran [148,149]. Es zeigt sich beispielsweise, daß die Wassermoleküle an der Grenzschicht eine deutliche Ordnung aufweisen. Dabei sind die Dipole des Wassers vorzugsweise parallel zur Membrannormalen ausgerichtet und weisen in Richtung der Membranoberfläche [150,151,152]. Von Tielemann und Mitarbeitern konnte die Membran entlang ihrer Normalen in vier verschiedene Regionen eingeteilt werden [153]. Die unterschiedlichen Hydratisierungsgrade der Kopfgruppen innerhalb der verschiedenen Lipidphasen konnten ebenso nachgewiesen werden $[154,155]$. Der Übergang von in der Grenzschicht gefangenem Wasser zur reinen Wasserphase erstreckt sich danach in der flüssigkristallinen Phase über einen wesentlich längeren Raumabschnitt als in der gelförmigen Phase. Das begründet das Konzept einer gebundenen Wasserphase vor allem oberhalb des Hauptphasenübergangs. Leider sind keine molekulardynamischen Simulationen der Rippled-Phase bekannt. Desweiteren wurde gefunden, daß die Wassermoleküle an der Grenzschicht die Möglichkeit haben, ungehindert entlang der Grenzfläche zu diffundieren [150,151,152]. Dies ist im Einklang mit den oben vorgestellten experimentellen Ergebnisse von Vöhringer und Mitarbeitern. Die freie Rotationsdiffusion scheint in reinem Wasser und der Grenzschicht offensichtlich auf die gleiche Weise stattzufinden. Die in MD-Simulationen gefundenen Störungen des Wasserstoffbrückennetzwerkes an Lipidgrenzflächen führen aber dazu, daß kollektive Translationsmoden nur in reinem Wasser und unterhalb des Vorphasenübergangs eine Rolle spielen [150,151,152]. 


\subsection{Farbstoffe}

In dieser Arbeit wurden verschiedene Farbstoffsonden zur Untersuchung der ultraschnellen Solvatation an biologischen Grenzschichten herangezogen, welche im folgenden kurz vorgestellt werden sollen. Dabei soll insbesondere auf ihre Vorund Nachteile im konkreten Einsatzfall eingegangen werden.

\subsubsection{Laurdan}

Für die Untersuchung der ultraschnellen Solvatationsdynamik in Lipidvesikeln mit Hilfe des zeitaufgelösten Stokes-Shifts wurde in der vorliegenden Arbeit der Farbstoff Laurdan (6-Lauroyl-2-dimethylaminonaphthalin) verwendet. Es handelt sich dabei um ein Derivat des 1979 von Weber und Farris synthetisierten Prodans (6-Propionyl-2-dimethylaminonaphthalin) [156] und unterscheidet sich von diesem lediglich in einer verlängerten Alkylkette (siehe Abbildung 3.5). Die Chromophore beider Farbstoffsonden sind identisch. Die maximalen Extinktionskoeffizienten liegen dabei in einem Bereich von $\epsilon=20000 \mathrm{~L} \mathrm{~mol}^{-1} \mathrm{~cm}^{-1}$.<smiles>CCC(=O)c1ccc2cc(N(C)C)ccc2c1</smiles>

Prodan<smiles>CCCCCCC(=O)c1ccc2cc(N(C)C)ccc2c1</smiles>

Laurdan

Abbildung 3.5: Chemische Struktur der Farbstoffe Prodan und Laurdan.

Die spektroskopischen Eigenschaften beider Farbstoffe reagieren sehr empfindlich auf die Polarität ihrer Umgebung. So befindet sich das Absorptionsmaximum für Prodan in Wasser bei $531 \mathrm{~nm}$, während es in Cyclohexan bei $401 \mathrm{~nm}$ liegt. Der dabei beobachtete stationäre Stokes-Shift beläuft sich auf $8640 \mathrm{~cm}^{-1}$ (Wasser) bzw. $4300 \mathrm{~cm}^{-1}$ (Cyclohexan) [156, 157, 158]. Diese ausgeprägte Solvatochromie wird durch ihre spezielle chemische Struktur hervorgerufen: Sie besitzen einen effektiven Elektronendonor (Dimethylaminogruppe), der über ein aromatisches System in Konjugation mit einem effektiven Elektronenakzeptor (Carbonylgruppe) steht. Die Änderung des Dipolmoments von Prodan bei elektronischer Anregung wurde in der Literatur ausgiebig diskutiert und schwankt dabei in weiten Bereichen von $\Delta \mu=20 \mathrm{D}$ bei Weber et al. bis $\Delta \mu=7-8 \mathrm{D}$ bei den übrigen Autoren, welche relativ konsistente Aussagen liefern [156,157,159,160,161]. Das Dipolmoment än- 
dert sich nach diesen Arbeiten von 2.9-4.7 D im Grundzustand auf 9.8-11.7 D im angeregten Zustand. Die Dipolmomente für Laurdan sind denen des Prodans sehr ähnlich [162]. Aufgrund dieser großen Dipolmomentsänderung bei elektronischer Anregung kann davon ausgegangen werden, daß in dem hier betrachteten DMPC/Wasser/Laurdan-System dipolare Solvatationsmechanismen dominierend sind, weswegen im folgenden in diesem System stets die dipolare Solvatation gemeint ist. Desweiteren wurde die Beteiligung von Wasserstoffbrückenbindungen bei der Solvatation diskutiert. Unabhängig von der genauen Natur der Solvatochromie geht die elektronische Anregung des Chromophors in polaren Umgebungen mit einem extrem großen Stokes-Shift einher. Das macht ihn zu einer geeigneten Farbstoffsonde in Experimenten, die den zeitaufgelösten Fluoreszenz Stokes-Shift ausnutzen, um Solvatationsdynamik zu messen.

Die ausgeprägte Empfindlichkeit auf die Polarität seiner Umgebung hat Prodan in den letzten Jahrzehnten zu einem weit verbreiteten Chromophor bei der Untersuchung von Lipidmembranen gemacht. In Lipidmembranen ist, wie bereits angemerkt, die Dielektrizitätskonstante eine sich stark ändernde Funktion entlang der Membrannormalen. Daher ist es von grundlegender Bedeutung, die genaue Lokalisierung der verwendeten Farbstoffsonde in der Membran zu kennen, da die Solvatationsdynamik eine Funktion des Ortes ist. Es zeigte sich aber, daß Prodan keine genau definierte Position in der Membran besitzt. Diskutiert werden zwei verschiedene Einbaulagen, die sich in einem bimodalen Emissionsverhalten ausdrücken [163]. Aus diesem Grund wurde Laurdan entwickelt, um eine genau definierte nicht-kovalente Verankerung des Chromophors über die Alkylkette in der Membran zu gewährleisten [164]. Laurdan scheint durch die Verankerung allerdings etwas tiefer in der Membran zu sitzen als Prodan, wodurch es einer etwas weniger polaren Umgebung ausgesetzt ist $[139,142,165]$. In Abbildung 3.6 ist die vermutete Einbaulage des Farbstoffes in einer DMPC-Membran schematisch dargestellt [165].

Von Parasassi et al. wurde die Beweglichkeit des Laurdans innerhalb einer Membranumgebung untersucht und diskutiert $[140,142,166]$. Diese Arbeiten gehen davon aus, daß trotz der tiefen Einbaulage des Chromophors vorrangig die Relaxation des Wassers an der Grenzfläche und nicht die Reorientierung der Farbstoffsonde ursächlich für den beobachteten Stokes-Shift ist.

Darüberhinaus wird in der Literatur die Beteiligung von Charge-Transfer-Zuständen (CT) bei den photophysikalischen Prozessen des Prodans und Laurdans kontrovers diskutiert [142,160,167,168,169,170,171]. Inwiefern verschiedene Einbaulagen und CT-Zustände die spektroskopischen Eigenschaften von Prodan und Laurdan beeinflussen, ist noch immer Gegenstand aktueller Forschung. Klar ist jedoch, daß ein bimodales Absorptions- und Emissionsverhalten des Chromophors beobachtet wird [165], was die Interpretation der Fluoreszenzspektren deutlich erschwert. 


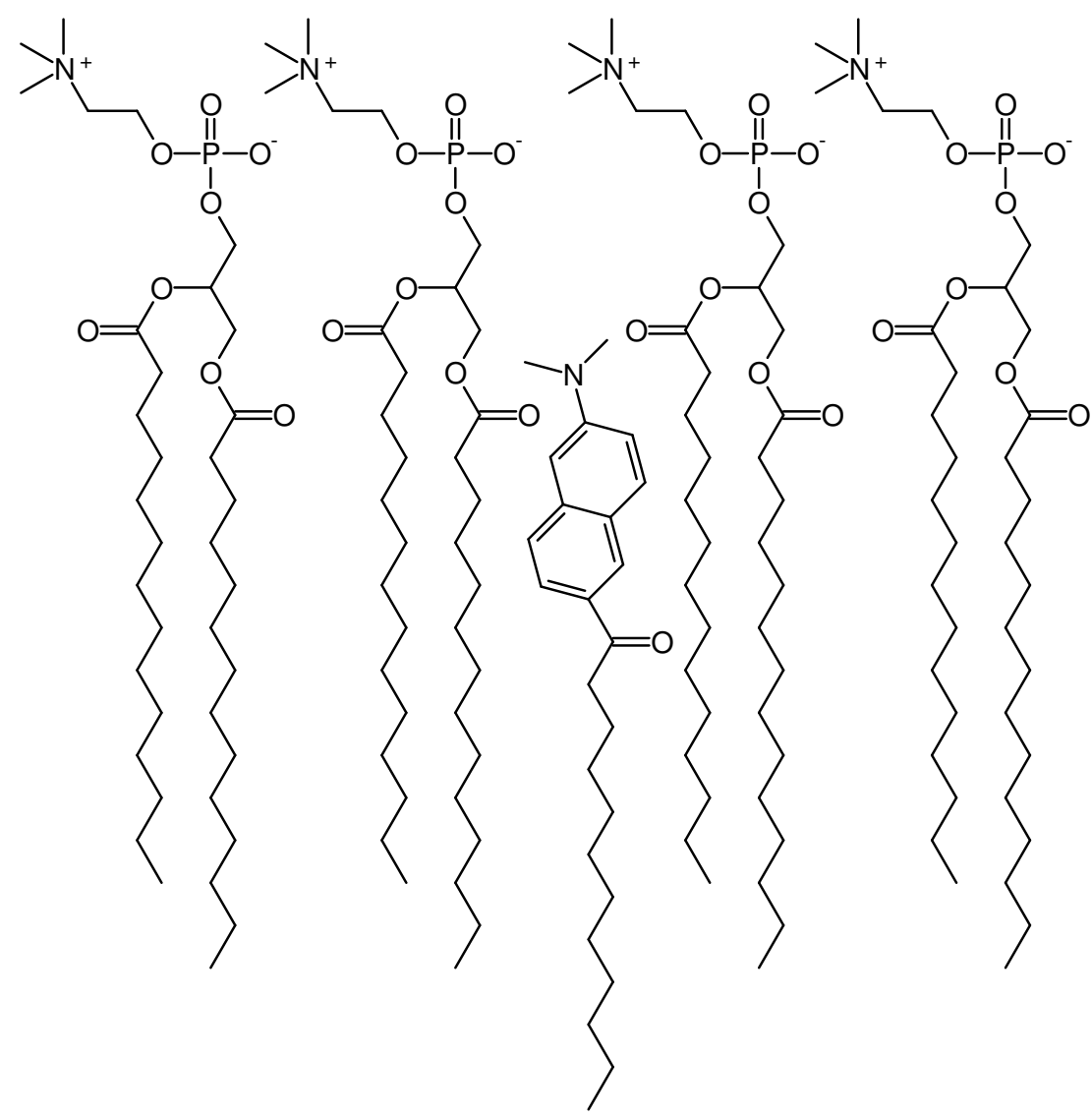

Abbildung 3.6: Schema einer mit der Farbstoffsonde Laurdan markierten DMPCMembran.

Zusammenfassend läßt sich feststellen, daß Laurdan aufgrund seiner ausgeprägten Solvatochromie und des großen Stokes-Shifts, sowie seiner komplexen Photophysik, eine äußerst interessante Farbstoffsonde für die Untersuchung der ultraschnellen Solvatation in Membranen über den zeitaufgelösten Stokes-Shift darstellt. Für diese Zwecke ist es derzeit eines der geeignetsten, kommerziell erhältlichen Farbstoffmoleküle. 


\subsubsection{HIDCI}

Für die Untersuchung der ultraschnellen Solvatationsdynamik von Wasser in inversen Mizellen und des reinen Wassers mit Hilfe der Photon-Echo Spektroskopie wurde der Farbstoff HIDCI (1, 1', 3, 3, 3', 3'-Hexamethylindodicarbocyaniniodid) eingesetzt. In der Literatur wird er auch als Hexacyanin 2, DiDC $_{1}$ oder C1-DiD bezeichnet.

Bei HIDCI handelt es sich um einen kommerziellen Laserfarbstoff [172]. Seine chemische Struktur ist in Abbildung 3.7 dargestellt.

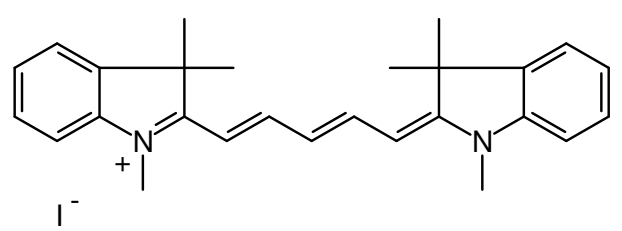

Abbildung 3.7: Chemische Struktur des Farbstoffes HIDCI.

Der Farbstoff zeichnet sich durch einen extrem großen Extinktionskoeffizienten von $\epsilon=225000 \mathrm{~L} \mathrm{~mol}^{-1} \mathrm{~cm}^{-1}$ im Maximum der Absorption aus. Dagegen weist er nur einen sehr geringen stationären Stokes-Shift auf, was ihn für die Untersuchungen der Solvatationsdynamik über den zeitaufgelösten Stokes-Shift ausschließt. Allerdings beeinflußt dieser geringe Stokes-Shift nicht die Verwendbarkeit im 3-Puls Photon-Echo Peakshift Experiment. Aufgrund seiner einfach negativen Ladung ist er in Wasser löslich und macht ihn zusammen mit seinem außerordentlichen Extinktionskoeffizienten zu einer geeigneten Farbstoffsonde für die Photon-Echo-Spektroskopie in Wasser. Entsprechend hat sich der Chromophor bereits vielfach für diese Art der Spektroskopie auch in biologischen Umgebungen bewährt $[27,50,79]$.

Auch für HIDCI sind photophysikalische Besonderheiten wie im Falle des Laurdans zu erwarten. So wurde von Martini und Hartland für den Farbstoff HITCI, der sich von HIDCI lediglich in einer um eine Doppelbindung verlängerten Polymethin-Kette unterscheidet, die Rotation um eine der Doppelbindungen im angeregten Zustand unter Bildung eines Photoisomers beobachtet [173, 174]. Da das 3-Puls Photon-Echo Peakshift Experiment allerdings nicht auf solche Prozesse empfindlich ist (siehe Kapitel 2.3), ergeben sich daraus keinerlei Schwierigkeiten bezüglich der Interpretation der experimentellen Ergebnisse. Für diese Arbeit spielen aber die durch elektronische Anregung induzierten Schwingungen eine Rolle, welche in Kapitel 6.4 eingehend besprochen werden. 


\section{Kapitel 4}

\section{Experimentelle Techniken}

In diesem Kapitel werden die angewendeten experimentellen Methoden zur Untersuchung der Solvatationsdynamik an biologischen Grenzschichten vorgestellt.

Dazu wird in Kapitel 4.1 zunächst auf die unterschiedlichen stationären Spektroskopiearten, sowie das kalorimetrische Verfahren eingegangen. Anschließend wird das zeitkorrelierte Einzelphotonenzählen beschrieben (Kapitel 4.2). Dieses Verfahren wurde zur Untersuchung der Solvatationsdynamik an Lipidmembran/Wasser-Grenzschichten im Nanosekundenbereich herangezogen. Kapitel 4.3 behandelt dann knapp das zur Durchführung der femtosekundenzeitaufgelösten Experimente verwendete kommerzielle Lasersystem. Dem im Rahmen dieser Arbeit neu aufgebauten Fluoreszenzkonversionsexperiment zur Untersuchung der ultraschnellen Solvatationsdynamik an Lipidmembran/WasserGrenzschichten ist das folgende Kapitel 4.4 gewidmet. Anschließend wird in Kapitel 4.5 das bei der Untersuchung der Solvatationsdynamik in inversen Mizellen verwendete Photon-Echo-Experiment beschrieben. Das abschließende Kapitel 4.6 geht in Kürze auf die verwendeten Chemikalien und die Probenpräparation ein.

\subsection{Stationäre Meßmethoden}

\subsubsection{Absorptionsspektroskopie}

Die in dieser Arbeit enthaltenen stationären Absorptionsspektren wurden mit einem UV-Vis-Spektrometer der Firma Shimadzu (UV 160) aufgenommen [175]. Für Messungen bei Zimmertemperatur wurden Quarzküvetten der Firma Hellma (110-QS) mit einer Schichtdicke von $1 \mathrm{~mm}$ verwendet. Die temperaturabhängigen Messungen wurden in thermostatierbaren Küvetten (Hellma, 165-QS) mit gleicher Schichtdicke durchgeführt. Die Temperaturen wurden dabei mit einem Thermostaten der Firma Julabo (Ultratemp 2000) auf $\pm 0.5^{\circ} \mathrm{C}$ konstant gehalten. 


\subsubsection{Emissions- und Anregungsspektroskopie}

Sämtliche Emissions- und Anregungsspektren wurden mit einem Fluoreszenzspektrometer der Firma Jobin Yvon - Spex Instruments S.A., Inc. (Fluorolog 3) und der zugehörigen Software Datamax aufgenommen [176]. Zur Anwendung kamen dabei die Küvetten 110-QS und 104.002-QS der Firma Hellma mit Schichtdicken von jeweils $10 \mathrm{~mm}$. Die Detektion der Fluoreszenz kann auf zweierlei Arten erfolgen. Die Standardvariante detektiert die Fluoreszenz im rechten Winkel zur Anregungsrichtung (RA, Right Angle Detection), wohingegen in einigen Fällen, d. h. bei stark absorbierenden Substanzen, eine Detektion unter einem Winkel von $22.5^{\circ}$ zur Anregungsrichtung notwendig war (FF, Front Face Detection). Welche Variante im jeweiligen Fall angewendet wurde, ist an entsprechender Stelle vermerkt. Zur Aufnahme temperaturabhängiger Spektren war eine geeignete thermostatierbare Küvettenhalterung mit angeschlossenem Thermostaten (Neslab, RTE-111) vorhanden. Die Temperatur konnte somit auf $\pm 1^{\circ} \mathrm{C}$ konstant gehalten werden.

\subsubsection{Dynamische Differentialkalorimetrie}

Zur Bestimmung von Wärmekapazitätsprofilen, z.B. zur Visualisierung von Phasenübergängen, dient das Verfahren der dynamischen Differentialkalorimetrie (DSC, Differential Scanning Calorimetry) [177]. Zur Verwendung kam ein VP-DSC Differentialkalorimeter der Firma MicroCal, Inc. (Northhampton/MA, USA). Probe- und Referenzzelle werden bei der DSC mit konstanter Heizrate $\Delta T / \Delta t$ erwärmt. Die dafür aufzubringenden Leistungen $P_{\text {Probe }}$ bzw. $P_{\text {Referenz }}$ hängen dabei von den in den Zellen ablaufenden Prozessen ab. Aus der Leistungsdifferenz $\Delta P=P_{\text {Probe }}-P_{\text {Referenz }}$ läßt sich die Wärmekapazitätsdifferenz $\Delta c_{p}$ berechnen:

$$
\Delta c_{p}=\left(\frac{\partial Q}{\partial T}\right)_{p} \simeq \frac{\Delta Q}{\Delta T} \simeq \frac{\Delta P}{\frac{\Delta T}{\Delta t}},
$$

dabei bezeichnet $\Delta Q$ die Wärmedifferenz, für welche bei kleinen $\Delta t$ gilt:

$$
\Delta Q=\int_{t}^{t+\Delta t} \Delta P \mathrm{~d} t \approx \Delta P \Delta t .
$$

\subsection{Zeitkorreliertes Einzelphotonenzählen}

Für die dynamischen Untersuchungen an Lipidmembran/Wasser-Grenzschichten im Nanosekundenbereich ist das zeitkorrelierte Einzelphotonenzählen (TCSPC, Time-Correlated Single Photon Counting) die Methode der Wahl [178]. Das zeitkorrelierte Einzelphotonenzählen nutzt die Tatsache aus, daß es sich bei der 
Emission von Photonen um einen rein statistischen Prozeß handelt. Die Anregung der Probe durch Laserimpulse möglichst hoher Repetitionsrate ist dabei so schwach zu halten, daß pro Laserimpuls die Detektionswahrscheinlichkeit eines Fluoreszenzphotons deutlich unter eins liegt (typischerweise $<0.001$ ). Werden die unter dieser Voraussetzung detektierten Photonen als Funktion der Zeit zwischen Anregung und Detektion in einem Histogramm erfaßt, so entspricht die erhaltene Verteilung dem zeitlichen Abklingverhalten der zu untersuchenden Fluoreszenz. Wird die Fluoreszenz zusätzlich frequenzaufgelöst detektiert, so erlaubt die Methode eine einfache Bestimmung des zeitabhängigen Fluoreszenz Stokes-Shifts (TDFSS, Time-Dependent Fluorescence Stokes-Shift) mit einem guten Signal/Rausch-Verhältnis (siehe Kapitel 5.4). Desweiteren wurden mit diesem System Messungen zur Anisotropie durchgeführt (siehe Kapitel 5.3).

\subsubsection{Experimenteller Aufbau}

Das verwendete Lasersystem besteht dabei aus einem Argon-Ionen-Laser (Coherent, Innova 410) gepumpten Titan:Saphir-Oszillator, welcher Pulse bei einer Zentralwellenlänge von $800 \mathrm{~nm}$, einer Repetitionsrate von $76 \mathrm{MHz}$ und einer Leistung von $300 \mathrm{~mW}$ liefert [179] (siehe Abbildung 4.1). Aus diesen Pulsen werden mittels einer Photodiode (Thorlabs) und einem Constant-Fraction-Discriminator mit integriertem Divider TTL-Steuerpulse mit einer Repetitionsrate von $38 \mathrm{MHz}$ erzeugt. Diese Repetitionsrate kann weiterhin mit einem zweiten Divider (Coherent 7200) variabel reduziert werden. Üblicherweise wird eine Teilrate von 20 verwendet, so daß schließlich Pulse von 1.9 MHz zur Verfügung stehen. Sie dienen als Steuerpulse für den akusto-optischen Modulator (Bragg-Zelle, $\mathrm{SiO}_{2}$-Kristall), der hier als Puls-Picker mit einer Auskopplungseffizienz von ca. $60 \%$ verwendet wird.

Die so erzeugten Laserpulse bei $800 \mathrm{~nm}$ und einer reduzierten Repetitionsrate von $1.9 \mathrm{MHz}$ werden mittels einer Prismenkombination (SF10, $\alpha=60^{\circ}$ ) zeitlich komprimiert und anschließend in einem nichtlinearen Kristall (BBO, $d=2 \mathrm{~mm}, \theta=31^{\circ}$ ) frequenzverdoppelt. Diese Laserpulse bei einer Zentralwellenlänge von $400 \mathrm{~nm}$ passieren eine $\lambda / 2$-Phasenverzögerungsplatte zur Einstellung des Polarisationszustandes und dienen als Anregungspulse für die zu untersuchenden Proben im TCSPC-Experiment. Sie werden mit einer Quarzlinse $(f=100 \mathrm{~mm})$ in eine thermostatierbare Zelle der Firma Hellma (165-QS, Schichtdicke $1 \mathrm{~mm}$ ) fokussiert. Die Temperatur in der Zelle konnte mit einem angeschlossenen Thermostaten (Julabo, Ultratemp 2000) auf $\pm 0.5^{\circ} \mathrm{C}$ konstant gehalten werden. Die in Rückwärtsrichtung abgestrahlte Fluoreszenz wird mit einer Kombination aus Linsen und Spiegeln zunächst kollimiert und anschließend in einen Monochromator im Czerny-Turner-Aufbau (AMKO, MULTImode 4, Brennweite $f=200 \mathrm{~mm}$, Gitter $=1200$ Linien $/ \mathrm{mm}, \lambda_{\text {Blaze }}=300 \mathrm{~nm}$, reziproke lineare Dispersion $=4 \mathrm{~nm} / \mathrm{mm})$ fokussiert $(f=150 \mathrm{~mm})$. Um aus den Anregungspulsen 


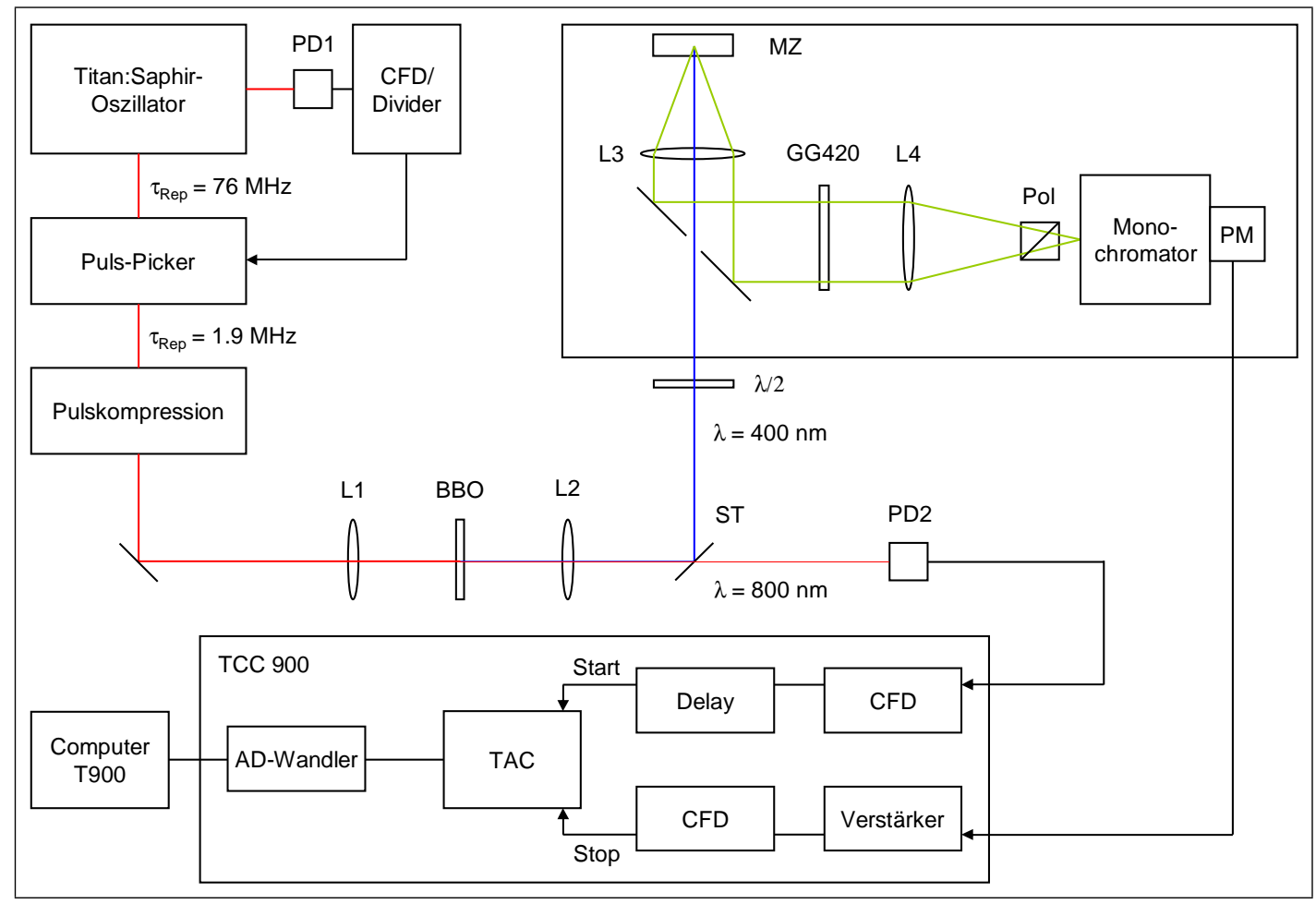

Abbildung 4.1: Schematischer Aufbau des zeitkorrelierten Einzelphotonenzählexperimentes. L1: $f=100 \mathrm{~mm}$, L2: $f=100 \mathrm{~mm}$, L3: $f=100 \mathrm{~mm}$, L4: $f=150 \mathrm{~mm}$, BBO: SHG-Kristall, ST: Strahlteiler, MZ: Meßzelle, Pol: Polarisator, PD1: Photodiode, PD2: Photodiode, PM: Photomultiplier; CFD: ConstantFraction-Discriminator, TAC: Zeit/Amplituden-Konverter.

herrührendes Streulicht zu unterdrücken wurde ein Grünglasfilter in den Strahlengang eingebracht (Schott, GG420, $1 \mathrm{~mm}$ ). Desweiteren wurde zur Vermeidung von Wood'schen Anomalien ein Polarisator vor den Eingang des Monochromators angebracht $[180,181,182]$. So ist sicher gestellt, daß lediglich parallel polarisiertes Licht in den Monochromator gelangt und so die störende Anomalie ausbleibt. Als Detektor am Ausgang des Monochromators dient ein Photomultiplier (Becker \& Hickl, PMH-100-1), der eine Antwortzeit von ca. 1.5 ns besitzt. Die erreichbare Zeitauflösung ist durch die unterschiedlichen Wege der erzeugten Elektronen von Dynode zu Dynode (Transient Time Spread) innerhalb des Photomultipliers begrenzt. Um sicherzustellen, daß lediglich Einzelphotonenereignisse stattfinden, um so die erforderliche Statistik zu erfüllen (Detektionswahrscheinlichkeit <0.001), wurde das Anregungslicht mit Neutralglasfiltern (Schott, NG1 und NG4, verschiedene Dicken) bei Bedarf entsprechend abgeschwächt. Zur Minimierung der Dunkelphotonenzählrate wurde die gesamte Detektionseinheit in einer lichtdichten Box untergebracht. 
Die gesamte Steuerung, Datenakquisition und Signalverarbeitung des TCSPCExperimentes erfolgt mit Hilfe einer PC-Einsteckkarte (Edinburgh Instruments Ltd., TCC 900) sowie der mitgelieferten Software T900 [183]. Sie vereint die notwendigen elektronischen Module für ein TCSPC-Experiment: ConstantFraction-Discriminator (CFD), elektronische Zeitverzögerungseinheiten (Delay), Zeit/Amplituden-Konverter (TAC, Time-to-Amplitude Converter), Verstärker, Analog/Digital-Wandler und digitalen Speicher. Die TCSPC-Karte TCC 900 verfügt über 2048 Kanäle. Der Startpuls für die elektronische Datenerfassung erfolgt über das unverdoppelte Restlicht der Anregungspulse, während die mit dem Photomultiplier nachgewiesene Fluoreszenz den Stoppuls darstellt. Die Messung erfolgte dabei im sogenannten Umkehrbetrieb (Reverse Modus), d. h. zunächst wurde der Stoppuls und erst anschließend relativ dazu der Startpuls detektiert. Für umfassende Behandlung des zeitkorrelierten Einphotonenzählens sei auf die umfassende Literatur bezüglich dieser Thematik hingewiesen [178, 184, 185].

\subsubsection{Charakterisierung des Meßsystems}

Da in dieser Arbeit die Fluoreszenzstrahlung vorrangig frequenzaufgelöst aufgenommen wurde, muß der spektralen Empfindlichkeit des Gesamtsystems Rechnung getragen werden. Die spektrale Antwort wurde dazu mit Hilfe einer Weißlichtquelle bekannten Spektrums (Osram 6318, $6 \mathrm{~V}, 10 \mathrm{~W}$ ) bestimmt, welche an Stelle der Meßzelle in den Strahlengang eingebracht wurde. Mit Hilfe der so gewonnen Kurve konnte eine spektrale Kalibration des Systems stattfinden. Sämtliche aus dem TCSPC-Experiment gewonnen und in dieser Arbeit präsentierten Ergebnisse wurden entsprechend korrigiert.

Um das zeitliche Auflösungsvermögen der Apparatur zu charakterisieren, wurde eine Gerätefunktion aufgenommen, welche der zeitlichen Instrumentenantwortfunktion des Gesamtsystems entspricht. Sie wurde anhand der Fluoreszenz einer DASPI/KI/MeOH-Lösung (DASPI: 4-[4-(Dimethylamino)-styryl]-1-methylpyridiniumiodid) bei $\lambda=615 \mathrm{~nm}$ bestimmt. Aufgrund der Fluoreszenzlöschung von DASPI durch Kaliumiodid, beträgt die Fluoreszenzlebensdauer ca. 50 ps [186] und liegt damit deutlich unterhalb der erreichbaren Zeitauflösung des TCSPCSystems. Das gemessene Signal sollte daher lediglich die Antwort des Meßsystems repräsentieren. Eine signifikante Abhängigkeit der Gerätefunktion von der Wellenlänge konnte nicht beobachtet werden. Die gemessene Antwortfunktion ist in Abbildung 4.2 zusammen mit einer Log-Normal-Anpassung [187] gemäß:

$$
g(t)=g_{0} \cdot \exp \left(-\ln 2 \cdot\left[\frac{\ln \left(1+\frac{2 b\left(t-t_{0}\right)}{\Delta}\right)}{b}\right]^{2}\right)
$$

dargestellt. Dabei bezeichnet $g_{0}$ die Amplitude, $b$ die Asymmetrie, $\Delta$ die Breite und $t_{0}$ den zeitlichen Nullpunkt. Eine Log-Normal-Funktion stellt eine asymme- 


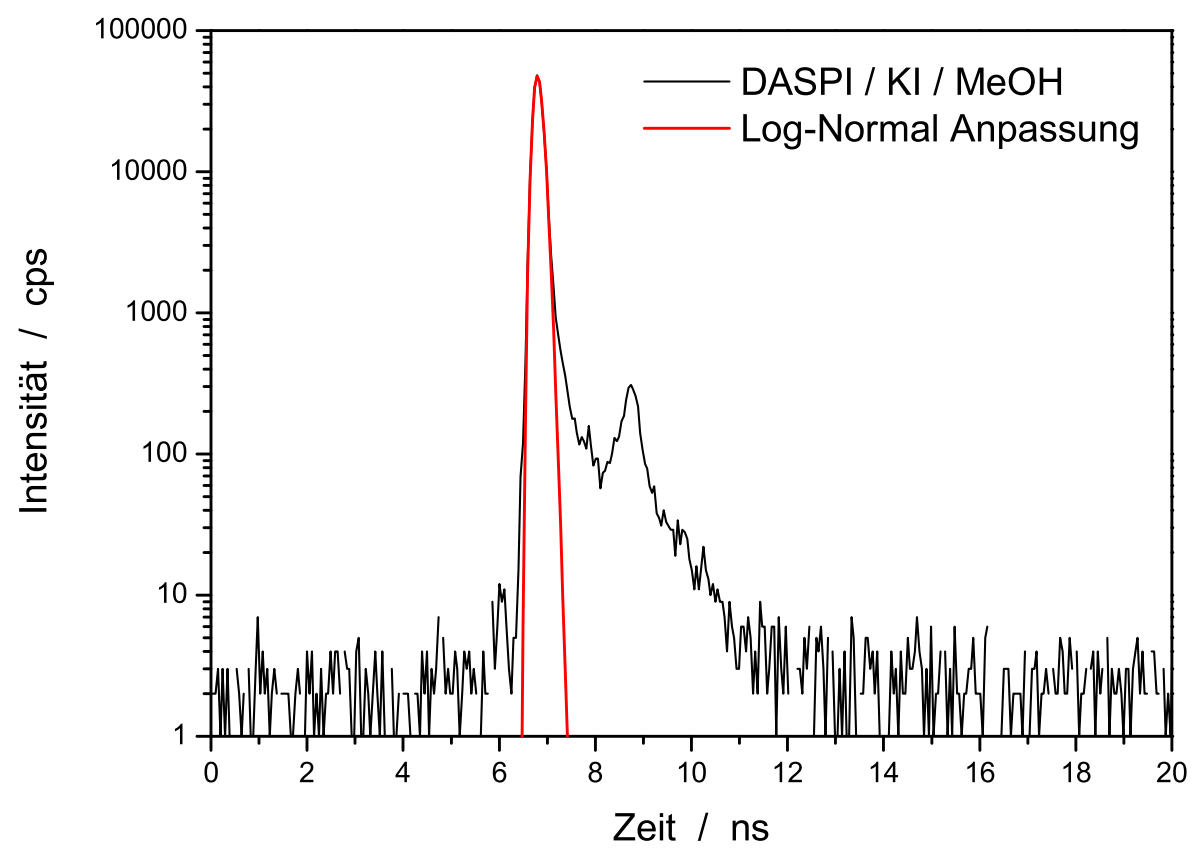

Abbildung 4.2: Gerätefunktion der TCSPC-Apparatur bei $\lambda=615 \mathrm{~nm}$ mit LogNormal-Anpassung.

trische Linienfunktion dar, die im Grenzfall $b \rightarrow 0$ in eine Gaußfunktion übergeht. Dabei gilt für die Halbwertsbreite $\tau_{\mathrm{FWHM}}$ (FWHM, Full Width at Half Maximum):

$$
\tau_{\mathrm{FWHM}}=\Delta\left(\frac{\sinh b}{b}\right)
$$

Die gemessene Antwortfunktion besitzt eine Halbwertsbreite von $\tau_{\mathrm{FWHM}}=223 \mathrm{ps}$, weswegen die Zeitauflösung der TCSPC-Apparatur auf ca. $200 \mathrm{ps}$ begrenzt ist. Desweiteren wurde anhand der Gerätefunktion der zeitliche Nullpunkt der Fluoreszenzabklingkurven zu $t_{0}=6.793$ ns bestimmt.

\subsubsection{Meßprozedur}

Für die Bestimmung des zeitabhängigen Fluoreszenz Stokes-Shifts wurden frequenzaufgelöste Fluoreszenzabklingkurven mit der TCSPC-Apparatur aufgenommen. Dies ermöglicht die Untersuchung der Solvatationsdynamik an Lipidmembran/Wasser-Grenzschichten auf einer Nanosekunden-Zeitskala. Von Interesse ist dabei insbesondere die Temperaturabhängigkeit der Solvatation, um die Dynamiken in unterschiedlichen Phasenzuständen der Membranen zu studieren. 
Dafür wurden für das System Laurdan/DMPC/Wasser $\left(c_{\mathrm{DMPC}}=10 \mathrm{mM}\right.$, $\left.n_{\text {DMPC }} / n_{\text {Laurdan }}=1000: 1\right)$ Kinetiken im Temperaturbereich von $T=5$ bis $60^{\circ} \mathrm{C}$ im Abstand von $5^{\circ} \mathrm{C}$ aufgenommen. Für jede dieser Temperaturen wurden Kinetiken von 400 bis $615 \mathrm{~nm}$ im Abstand von $5 \mathrm{~nm}$ gemessen. Die Fluoreszenzabklingkurven wurden über einen Zeitraum von 50 ns registriert, wobei die Datenakquisition für eine Kinetik über einen Zeitraum von 60 Sekunden stattfand. Bei dem gewählten Meßintervall von 50 ns beträgt der zeitliche Punktabstand ca. 25 ps. Die spektrale Auflösung ist bei der eingestellten Spaltbreite des Monochromators von $1 \mathrm{~mm}$ auf ca. $4 \mathrm{~nm}$ begrenzt. Die Anregung der Probe erfolgte im magischen Winkel von $54.7^{\circ}$ um Einflüsse des Polarisationszustandes auf die Fluoreszenzabklingkurven zu vermeiden $[158,188]$. Die registrierten Kinetiken wurden entsprechend ihrer spektralen Lage bezüglich ihrer Intensität korrigiert (siehe Kapitel 4.2.2). Eine Entfaltung der Kinetiken mit der Gerätefunktion fand nicht statt. Aus den so gewonnen Kinetiken konnte der zeitabhängige Fluoreszenz Stokes-Shift rekonstruiert werden (siehe Kapitel 5.4).

Für die Bestimmung der Anisotropieeigenschaften des Laurdans an der Lipidmembran/Wasser-Grenzschicht wurden für obige Temperaturen (und zusätzlich für $22.5^{\circ} \mathrm{C}$ ) für parallel $\left(0^{\circ}, \|\right)$ und senkrecht $\left(90^{\circ}, \perp\right)$ polarisiertes Anregungslicht bezüglich der Detektion Kinetiken in den jeweiligen Intensitätsmaxima der Fluoreszenz aufgenommen. Die Datenakquisition erfolgte über einen Zeitraum von jeweils 5 Minuten. Aus diesen Kinetiken konnte die Anisotropiefunktion berechnet werden (siehe Kapitel 5.3):

$$
r(t)=\frac{I_{\|}(t)-I_{\perp}(t)}{I_{\|}(t)+2 I_{\perp}(t)} .
$$

Bei diesen Messungen ist eine spektrale Korrektur nicht notwendig, da sie sich auf die gemessenen Kinetiken bei paralleler und senkrechter Anregung gleichermaßen auswirken würde. Eine Entfaltung mit der Gerätefunktion fand nicht statt.

\subsection{Laser für Ultrakurzzeit-Untersuchungen}

Als Laserlichtquelle für die Untersuchungen der Ultrakurzzeitdynamiken dient ein kommerzielles Lasersystem der Firma Clark-MXR Inc. (CPA-2001) basierend auf einem Faserlaser-Ring-Oszillator und regenerativem Verstärkersystem [189]. Dieses System liefert Laserpulse bei einer Zentralwellenlänge von $775 \mathrm{~nm}$ und einer Impulsdauer von ca. $150 \mathrm{fs}$. Die Repetitionsrate beträgt $1 \mathrm{kHz}$ bei einer Pulsenergie von $0.85 \mathrm{~mJ}$. Jeweils $25 \%$ der Energie dieser Laserpulse dienen als Ausgangspulse für das Fluoreszenzkonversionsexperiment (Kapitel 4.4) bzw. zum Betreiben des nichtkollinear optisch-parametrischen Verstärkers, welcher für das Photon-Echo-Experiment herangezogen wird (Kapitel 4.5). 
Der CPA-2001 ist ein modular aufgebautes Femtosekunden-Lasersystem, bestehend aus einem Diodenlaser-gepumpten Faserlaser-Ring-Oszillator, einem Pulsstrecker, einem regenerativen Verstärker und einem Pulskompressor. Der Diodenlaser $(\lambda=980 \mathrm{~nm}, P=900 \mathrm{~mW})$ wird mittels eines WDM-Kopplers (WDM, Wavelength Division Multiplexing) in die $\mathrm{Er}^{3+}$-dotierte Faser eingebunden. Dieser Faser-Oszillator emittiert bei $1550 \mathrm{~nm}$ und erreicht über polarisationsadditives Modenkoppeln (APM, Polarisation Additive Pulse Modelocking) stabilen Pulsbetrieb [27, 189, 190,191, 192]. Die im Ringlaser erzeugten Pulse werden anschließend mittels eines Gitters zeitlich auf ca. $100 \mathrm{fs}$ komprimiert und in einem Lithiumtriborat-Kristall (LBO) frequenzverdoppelt. Diese bei $775 \mathrm{~nm}$ zentrierten Laserpulse werden nun zeitlich gestreckt und sodann in den regenerativen Verstärker (RGA, Regenerative Amplifier) eingekoppelt [193, 194, 195, 196]. Als Verstärkungsmedium dient ein Titan:Saphir-Kristall, welcher mittels eines frequenzverdoppelten Nd:YAG-Lasers $(\lambda=532 \mathrm{~nm}, E=8 \mathrm{~mJ})$ bei einer Wiederholfrequenz von $1 \mathrm{kHz}$ gepumpt wird. Nach erfolgter Verstärkung bis zur Sättigung, wird der verstärkte Laserpuls über eine Pockels-Zelle aus dem Verstärker ausgekoppelt. Die zeitliche Kontrolle der Schaltvorgänge zum Ein- bzw. Auskoppeln erfolgt mittels eines Treibers (Clark-MXR Inc., DT505), welcher so eine optimale Verstärkung im RGA gewährleistet. Die Stabilität der Laserpulse und die Anzahl der Verstärkungszyklen werden dabei mittels einer Photodiode ständig kontrolliert und auf einem Oszilloskop (Tektronix 485, $350 \mathrm{MHz}$ ) visualisiert (Abbildung 4.3). Anschließend werden die verstärkten Laserpulse mittels eines Gitters zeitlich optimal komprimiert. Dieses Verfahren der Verstärkung wird als Chirped Pulse Amplification (CPA) bezeichnet.

Es wurden routinemäßig Messungen zur zeitlichen Charakterisierung der Laserpulse mittels eines dafür aufgebauten Autokorrelators durchgeführt. Die dabei detektierte Intensitätsautokorrelationsfunktion lautet:

$$
A(\tau)=\int_{-\infty}^{\infty} I(t) I(t+\tau) \mathrm{d} t
$$

An die in Abbildung 4.4 dargestellte, typische Intensitätsautokorrelationsfunktion wurde ein Lorentz-Profil angepaßt, wonach sich Pulsdauern im Bereich von $\tau_{\text {Imp }}=120 \mathrm{fs}$ ergeben. Der in der Messung zu erkennende Untergrund läßt sich durch höhere Beugungsordnungen resultierend aus der Gitterkompression im CPA-2001 erklären. 


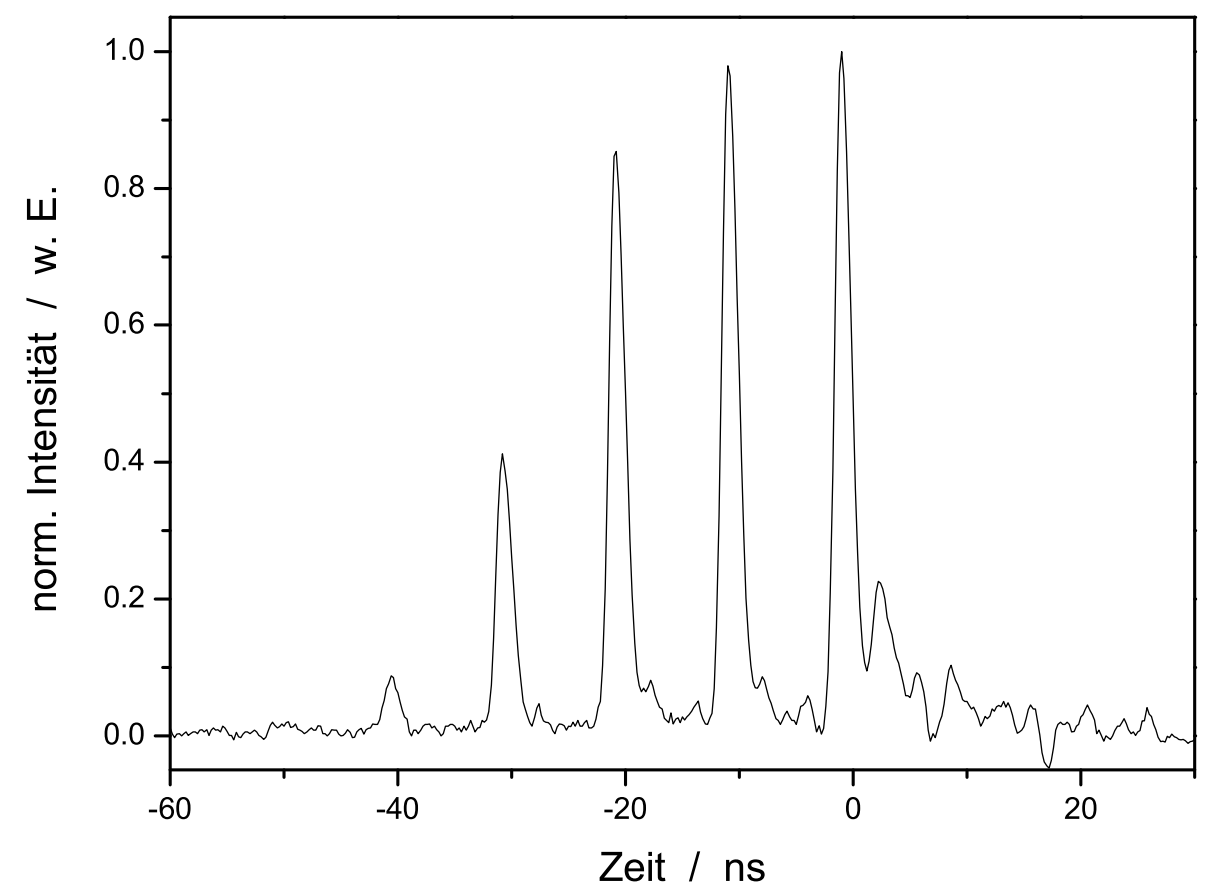

Abbildung 4.3: Verstärkung der Laserpulse im RGA.

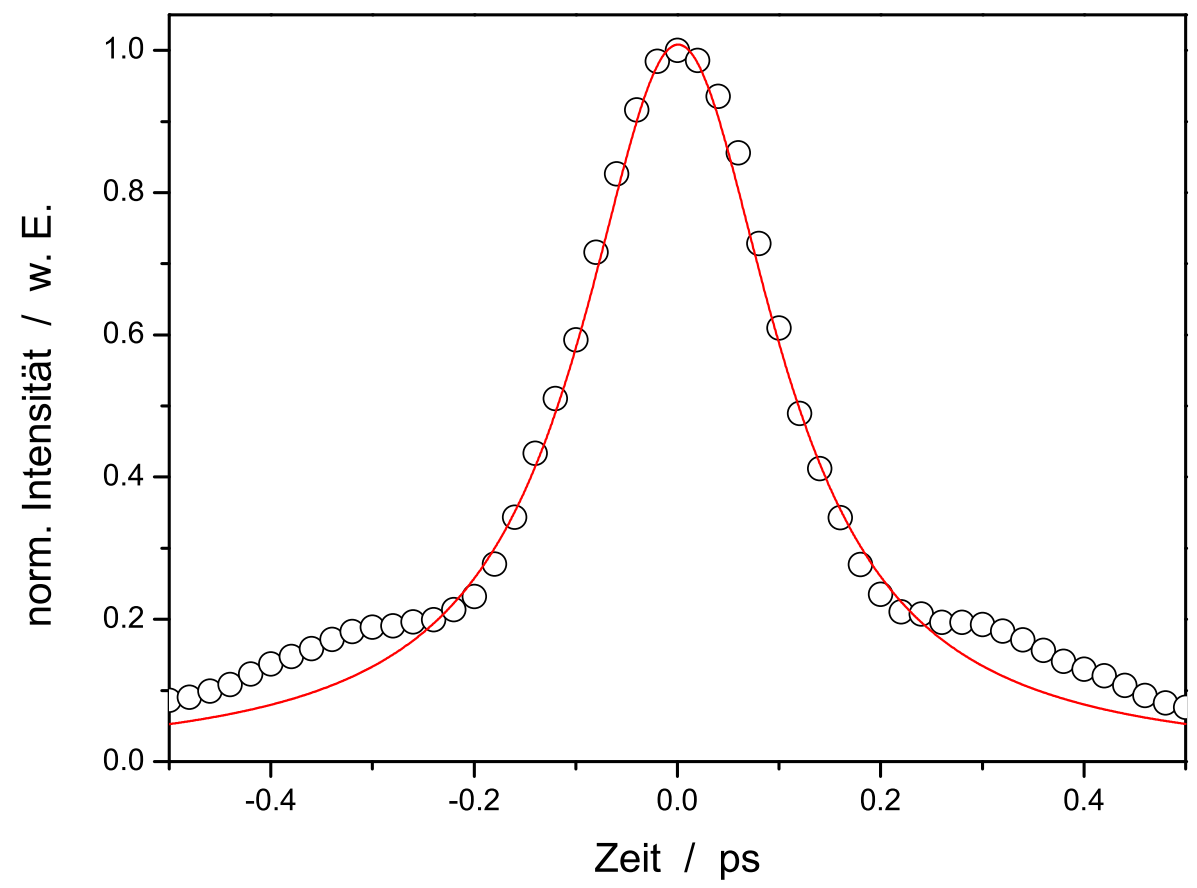

Abbildung 4.4: Autokorrelationsmessung der Laserpulse aus dem CPA-2001 mit lorentzförmiger Anpassung. 


\subsection{Fluoreszenzkonversionsspektroskopie}

Im Nanosekundenzeitbereich ist das zeitkorrelierte Einzelphotonenzählen die bevorzugte Methode zur Untersuchung zeitaufgelöster Fluoreszenzspektren. Die unterschiedlichen Wege der erzeugten Elektronen von Dynode zu Dynode (Transient Time Spread) des verwendeten Detektors stellen bei dieser Methode jedoch die Grenze der Zeitauflösung dar. Streak-Kameras erlauben zwar eine Zeitauflösung im Pikosekundenbereich, sollen allerdings noch schnellere Prozesse im Femtosekundenbereich untersucht werden, so wird mit den herkömmlichen Methoden schnell die Grenze des Möglichen erreicht.

Um solche ultraschnellen Prozesse untersuchen zu können, bedient man sich Samplingverfahren, die den bekannten Boxcar-Verfahren ähnlich sind [197, 198, 199]. Anstelle elektronischer Tore werden allerdings optisch-nichtlineare Tore eingesetzt. Ein bekanntes Beispiel stellt der sogenannte Kerr-Shutter dar. Er basiert auf der durch einen intensiven Laserstrahl induzierten Anisotropie in einem zuvor optisch isotropen Material (z. B. $\mathrm{CS}_{2}$ ). Durch geeignete Meßanordnungen läßt sich so die durch Laseranregung erzeugte Fluoreszenz zeitlich abtasten und detektieren [200,201,202]. Dem Vorteil, daß bei dieser Methode das gesamte Spektrum der Fluoreszenz simultan detektiert werden kann, steht der Nachteil gegenüber, daß der Prozeß sehr ineffizient ist und man daher auf hohe Fluoreszenzintensitäten angewiesen ist.

Im Jahre 1975 stellten Hirsch und Mahr die Methode der Fluoreszenzaufkonvertierung (FUC, Fluorescence Up-Conversion) vor [203], die es erlaubt sehr schwache Fluoreszenzintensitäten bei guter spektraler und hoher zeitlicher Auflösung nachzuweisen, welche prinzipiell nur durch die Länge der verwendeten Laserpulse limitiert ist.

\subsubsection{Prinzip der Fluoreszenzkonversionsspektroskopie}

Ein Fluoreszenzaufkonvertierungsexperiment stellt prinzipiell eine Kreuzkorrelation zwischen Fluoreszenzlicht und einem intensiven Laserpuls dar [204, 205, 206, 207,208,209,210]. Die zu untersuchende Probe wird mit einem Laserpuls der Frequenz $\omega_{\text {Pump }}$ elektronisch angeregt und die dabei entstehende inkohärente Fluoreszenz der Frequenz $\omega_{\mathrm{Fl}}$ wird gesammelt und zusammen mit einem intensiven Laserpuls, dem sogenannten Gatepuls („Torpuls“) der Frequenz $\omega_{\text {Gate }}$, in einen optisch-nichtlinearen Kristall fokussiert. Sind die Phasenanpassungsbedingungen erfüllt, so entsteht bei zeitlicher und räumlicher Überlagerung beider Wellen im Kristall die Summen- bzw. Differenzfrequenz $\omega_{\mathrm{S}}$. Bei Erzeugung der Summenfrequenz wird das Experiment als Up-Conversion bezeichnet, im anderen Fall als Down-Conversion. 
Die Phasenanpassungsbedingungen für die Summenfrequenzerzeugung, die in dieser Arbeit verwendet wurde, lauten für die Fluoreszenzaufkonvertierung:

$$
\begin{aligned}
& \omega_{\mathrm{S}}=\omega_{\mathrm{Fl}}+\omega_{\text {Gate }} \quad \text { bzw. } \\
& \vec{k}_{\mathrm{S}}=\vec{k}_{\mathrm{Fl}}+\vec{k}_{\text {Gate }}
\end{aligned}
$$

Dabei bezeichnen $\vec{k}_{\mathrm{S}}, \vec{k}_{\mathrm{Fl}}$ und $\vec{k}_{\text {Gate }}$ die jeweiligen Wellenvektoren. Es handelt sich bei diesen Bedingungen um die durch $\hbar$ dividierten Erhaltungssätze der Energie und des Impulses.

Da die Summenfrequenz nur dann erzeugt wird, wenn sich Fluoreszenz und Pumppuls gleichzeitig im Kristall überlagern, kann nun der zeitliche Fluoreszenzintensitätsverlauf erfaßt werden, indem der Gatepuls bezüglich der Fluoreszenz zeitlich variiert und somit nacheinander der gesamte Fluoreszenzverlauf abgetastet wird (siehe dazu Abbildung 4.5 zum Prinzip eines FUC-Experimentes). Dies ist möglich, weil die Intensität der Summenfrequenz proportional zur Korrelationsfunktion der Fluoreszenzintensität mit der Intensität des Gatepulses ist:

$$
I_{\mathrm{S}}(\tau) \propto \int_{-\infty}^{\infty} I_{\mathrm{Fl}}(t) I_{\mathrm{Gate}}(t-\tau) \mathrm{d} t .
$$

Der Gatepuls fungiert somit als ein zeitliches Tor, wodurch die Fluoreszenzkonversionsspektroskopie das optische Analogon zur elektronischen Boxcartechnik darstellt.

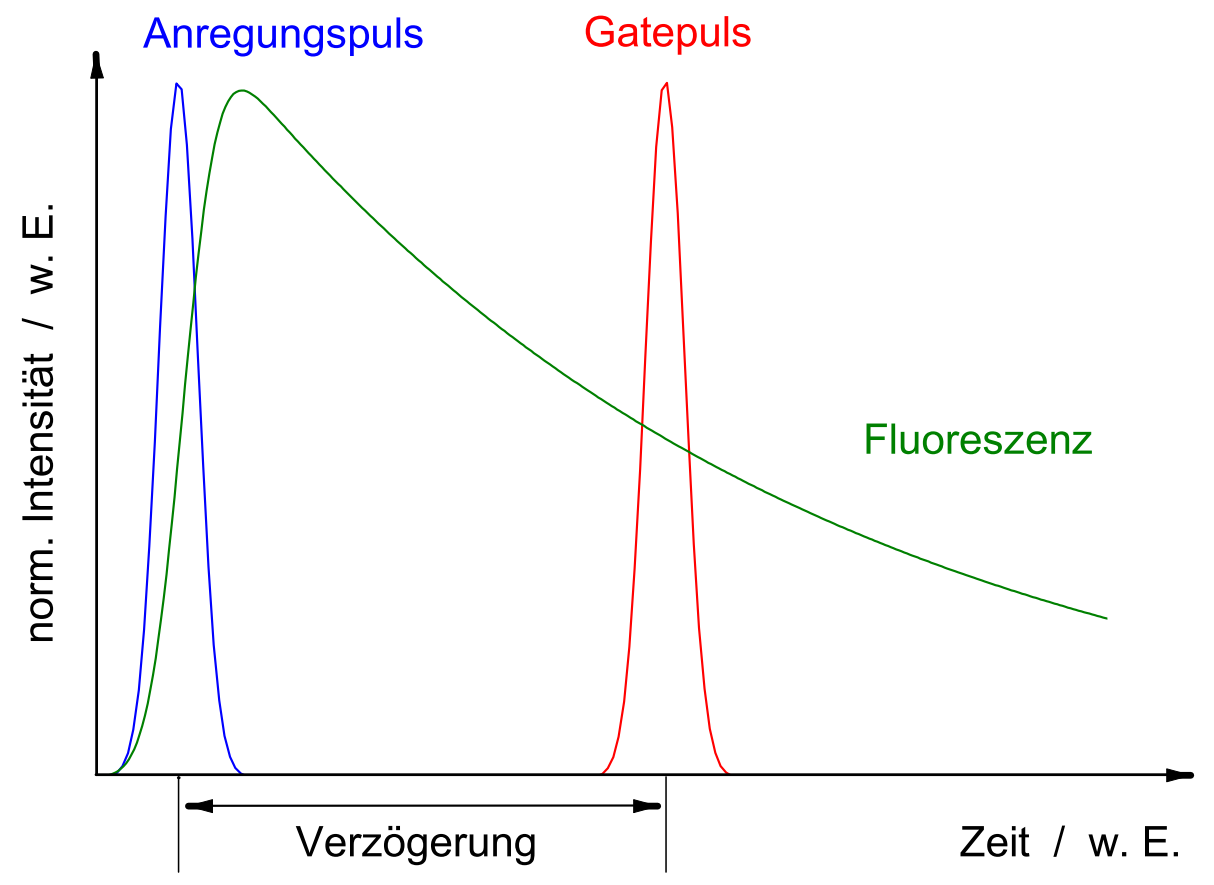

Abbildung 4.5: Prinzip eines Fluoreszenzkonversionsexperimentes. 


\section{Realisierung der Phasenanpassungsbedingungen}

Im Falle der kollinearen Phasenanpassung, wie sie in dieser Arbeit verwendet wurde, reduziert sich Gleichung $4.8 \mathrm{zu}$ :

$$
\frac{n_{\mathrm{S}}}{\lambda_{\mathrm{S}}}=\frac{n_{\mathrm{Fl}}}{\lambda_{\mathrm{Fl}}}+\frac{n_{\text {Gate }}}{\lambda_{\text {Gate }}}
$$

wobei $n$ die jeweiligen Brechungsindizes bei den entsprechenden Wellenlängen $\lambda$ bezeichnen [205]. Ein Weg diese Phasenanpassungsbedingung zu erfüllen besteht darin, die Doppelbrechungseigenschaften von anisotropen, häufig uniaxialen Kristallen auszunutzen. Sie zeichnen sich dadurch aus, daß die Brechungsindizes von der Ausbreitungsrichtung und Polarisation der einfallenden Welle bezüglich der optischen Achse $\vec{c}$ abhängen. Die beiden Wellen werden als ordentliche (o) bzw. außerordentliche (ao) Wellen bezeichnet, wobei beide Wellen senkrecht zueinander polarisiert sind und deren Brechungsindizes mit $n_{\mathrm{o}}$ bzw. $n_{\text {ao }}$ bezeichnet werden.

In einem uniaxialen Kristall (d.h. Kristalle mit nur einer optischen Achse), wie dem in dieser Arbeit verwendeten $\beta$-Bariumborat-Kristall (BBO), gilt $n_{x}=n_{y}=n_{\mathrm{o}}$ bzw. $n_{z}=n_{\text {ao }}$, wenn $z$ die Richtung der optischen Achse $\vec{c}$ bezeichnet. Daraus folgt:

$$
\frac{1}{n_{i}^{2}(\theta)}=\frac{\sin ^{2} \theta}{n_{\mathrm{ao}, i}^{2}}+\frac{\cos ^{2} \theta}{n_{\mathrm{o}, i}^{2}}
$$

wenn $\theta$ den Einfallswinkel des Lichtes zur optischen Achse beschreibt und $i$ für die unterschiedlichen Wellen steht (S, Fl und Gate).

Die Phasenanpassungsbedingungen (Gleichung 4.7 bzw. 4.10) lassen sich weiterhin nicht erfüllen, wenn alle drei Wellen im Kristall die gleiche Polarisation besitzen. Unter der Bedingung, daß einer der Strahlen als außerordentliche Welle durch den Kristall propagiert, läßt sich jedoch ein Winkel $\theta_{\mathrm{m}}$ finden, für den die Phasenanpassungsbedingungen erfüllt sind.

Beim verwendeten BBO-Kristall handelt es sich um einen negativ uniaxialen Kristall, d. h. es gilt $n_{\text {ao }}<n_{\mathrm{o}}$. Bei genauerer Betrachtung läßt sich zeigen, daß bei solchen Kristallen die Phasenanpassung nur zu erreichen ist, wenn die erzeugte Summenfrequenz eine außerordentliche Welle ist. Es werden zudem zwei verschiedene Typen der Phasenanpassung unterschieden. Im Falle der sogenannten Typ I- Phasenanpassung sind die beiden einfallenden Lichtwellen gleicher Polarisation $(\mathrm{o}+\mathrm{o} \rightarrow \mathrm{ao})$, wohingegen im Falle der Typ II-Phasenanpassung die einfallenden Wellen orthogonal zueinander polarisiert sind $(\mathrm{o}+\mathrm{ao} \rightarrow$ ao bzw. $\mathrm{ao}+\mathrm{o} \rightarrow \mathrm{ao})$.

Für den in dieser Arbeit benutzen Fall der Typ I - Phasenanpassung läßt sich nun der gesuchte Phasenanpassungswinkel $\theta_{\mathrm{m}}$ berechnen:

$$
\sin ^{2} \theta_{\mathrm{m}}=\frac{n_{\mathrm{S}}^{-2}\left(\theta_{\mathrm{m}}\right)-n_{\mathrm{o}, \mathrm{S}}^{-2}}{n_{\mathrm{ao}, \mathrm{S}}^{-2}-n_{\mathrm{o}, \mathrm{S}}^{-2}},
$$


wobei $n_{\mathrm{S}}\left(\theta_{\mathrm{m}}\right)$ gegeben ist als:

$$
n_{\mathrm{S}}\left(\theta_{\mathrm{m}}\right)=n_{\mathrm{o}, \mathrm{Fl}} \frac{\lambda_{\mathrm{S}}}{\lambda_{\mathrm{Fl}}}+n_{\mathrm{o}, \text { Gate }} \frac{\lambda_{\mathrm{S}}}{\lambda_{\mathrm{Gate}}} .
$$

Für diesen Winkel $\theta_{\mathrm{m}}$ bezüglich der optischen Achse des Kristalls sind die geforderten Phasenanpassungsbedingungen exakt erfüllt.

Die Umwandlungseffektivität $\eta_{\mathrm{q}}$ für die Summenfrequenzerzeugung läßt sich für diesen Fall unter Anwendung einiger Näherungen angeben als:

$$
\eta_{\mathrm{q}}=\frac{I_{\mathrm{S}}}{I_{\text {Gate }}}=\frac{2 \pi^{2} d_{\mathrm{eff}}^{2} L^{2} I_{\mathrm{Fl}}}{c \varepsilon_{0}^{3} \lambda_{\mathrm{Fl}} \lambda_{\mathrm{S}} n_{\mathrm{o}, \mathrm{Fl}} n_{\mathrm{o}, \text { Gate }} n_{\mathrm{S}}\left(\theta_{\mathrm{m}}\right)} .
$$

Dabei bezeichnet $I$ die jeweiligen eingestrahlten Intensitäten, $d_{\text {eff }}$ den effektiven nichtlinearen Koeffizienten des Kristalls, $L$ die Dicke des Kristalls, $c$ die Lichtgeschwindigkeit und $\varepsilon_{0}$ die absolute Dielektrizitätskonstante des Vakuums [206].

\section{Akzeptanzwinkel und spektrale Bandbreite}

Die obigen Ableitungen gelten streng nur für die Phasenanpassung $\Delta \vec{k}=\vec{k}_{\mathrm{S}}-$ $\vec{k}_{\mathrm{Fl}}-\vec{k}_{\text {Gate }}=0$. Sie ist jedoch nicht immer exakt erfüllt.

Die durch den Pumppuls erzeugte Fluoreszenz strahlt in alle Raumrichtungen ab und kann folglich auch nur mit einem bestimmten Raumwinkel in den Kristall fokussiert werden, so daß die in den Kristall eintretende Fluoreszenz mit den Wellenvektoren $\vec{k}_{\mathrm{Fl}}$ eine starke Streuung der Einstrahlungswinkel aufweist [207]. Es kann also keine exakt kollineare Einstrahlungsgeometrie für die gesamte Fluoreszenz gewährleistet werden. Diese Umstände führen dazu, das es zu einer Situation der sogenannten Phasenfehlanpassung (Phase Mismatch) kommt, d.h. $\Delta \vec{k}=\vec{k}_{\mathrm{S}}-\vec{k}_{\mathrm{Fl}}-\vec{k}_{\text {Gate }} \neq 0$. Das hat negative Auswirkungen auf die Umwandlungseffektivität im Kristall [206,207,208], sie reduziert sich zu:

$$
\eta_{\mathrm{q}}(\Delta \vec{k})=\eta_{\mathrm{q}} \cdot \frac{\sin ^{2}(\Delta \vec{k} L / 2)}{(\Delta \vec{k} L / 2)^{2}} .
$$

Eine effektive Umwandlungseffektivität kann daher nur innerhalb eines gewissen Akzeptanzwinkels des Kristalls erfolgen. Dieser Akzeptanzwinkel $\Delta \theta$ ist definiert als der Winkel in der die Phasenfehlanpassung weniger als $\pi / 2$ beträgt. Es gilt:

$$
\Delta \theta=\frac{4 \pi}{L}\left(\frac{\partial \Delta \vec{k}}{\partial \theta}\right)^{-1}
$$

Desweiteren führt die große spektrale Bandbreite der Fluoreszenz dazu, daß die Phasenanpassungsbedingungen im Kristall nicht immer für alle Strahlungsanteile 
perfekt erfüllt sein können. Analog den obigen Überlegungen kann für die spektrale Bandbreite $\Delta \omega$ des Kristalls definiert werden [207]:

$$
\Delta \omega=\frac{\pi}{2 L\left(\frac{\partial \vec{k}_{\mathrm{S}}}{\partial \omega_{\mathrm{S}}}-\frac{\partial \vec{k}_{\mathrm{Fl}}}{\partial \omega_{\mathrm{Fl}}}\right)} .
$$

Sie umfaßt den Bereich, in dem die Energie des aufkonvertierten Lichtes auf $4 / \pi^{2}$ der ursprünglichen Intensität abgesunken ist. Der Kristall wirkt also als ein frequenzselektives Element.

\section{Zeitauflösung}

Die Zeitauflösung in einem FUC-Experiment ist prinzipiell durch die zeitliche Breite des Pump- und vorrangig des Gate-Pulses gegeben [204,209].

Bei Messungen im Femtosekundenbereich kann diese minimal erreichbare Zeitauflösung durch die sogenannte Gruppengeschwindigkeitsdispersion (GVD, Group Velocity Dispersion) verschlechtert werden. Diese Gruppengeschwindigkeitsdispersion resultiert aus den optischen Elementen im Strahlengang und kann beschrieben werden als:

$$
\mathrm{GVD}=\frac{\partial^{2} \vec{k}}{\partial \omega^{2}}=\frac{\lambda^{3}}{2 \pi c^{2}} \frac{\partial^{2} n}{\partial \lambda^{2}}
$$

Die GVD bewirkt, daß die Pulse einen sogenannten Chirp besitzen, d.h. die niederfrequenten Strahlungsanteile eilen den höherfrequenten zeitlich voraus, so daß die Pulse zeitlich verlängert und in der Frequenz "verschmiert" sind. Der störende Effekt der GVD kann weitestgehend umgangen werden, indem reflektive Optiken verwendet werden, d. h. Parabolspiegel anstatt Linsen.

Ein weiteres Problem stellt die sogenannte Gruppengeschwindigkeitsfehlanpassung (GVM, Group Velocity Mismatch) dar. Sie rührt aus unterschiedlichen Fortbewegungsgeschwindigkeiten der einzelnen Frequenzen im Aufkonvertierungskristall her. Unterschiedliche Frequenzen laufen bildlich gesprochen aneinander vorbei und bewirken so die zeitliche Verbreiterung der erzeugten Summenfrequenz. Die Gruppengeschwindigkeit $v_{\mathrm{g}}$ bei einer Wellenlänge $\lambda_{0}$ wird beschrieben als:

$$
v_{\mathrm{g}}=\left(\frac{\partial \vec{k}}{\partial \omega}\right)^{-1}=c\left[n\left(\lambda_{0}\right)-\lambda_{0}\left(\frac{\partial n}{\partial \lambda}\right)_{\lambda_{0}}\right]^{-1} .
$$

Im Falle der hier verwendeten Typ I - Phasenanpassung kann die aus der Gruppengeschwindigkeitsfehlanpassung resultierende zeitliche Verbreiterung beschrieben werden als:

$$
\Delta t=L\left(\frac{\partial \vec{k}_{\mathrm{Gate}}}{\partial \omega_{\mathrm{Gate}}}-\frac{\partial \vec{k}_{\mathrm{Fl}}}{\partial \omega_{\mathrm{Fl}}}\right) .
$$


Um die zeitliche Verbreiterung durch die Gruppengeschwindigkeitsfehlanpassung möglichst gering zu halten, sollten möglichst dünne Aufkonvertierungskristalle eingesetzt werden. Es ist daher ein Kompromiß zwischen erreichbarer Zeitauflösung und Umwandlungseffizenz des Aufkonvertierungsprozesses zu finden.

\subsubsection{Experimenteller Aufbau}

Der schematische Versuchsaufbau des im Rahmen dieser Arbeit aufgebauten Fluoreszenzkonversionsexperimentes (FUC, Fluorescence Up-Conversion) ist in Abbildung 4.6 dargestellt. Das FUC-Experiment wird mit 25\% der aus dem CPA-2001 zur Verfügung gestellten Laserleistung betrieben. Der bei einer Wellenlänge von $775 \mathrm{~nm}$ zentrierte Laser wird zunächst mit einem Galilei-Teleskop um einen Faktor 3 bezüglich seines ursprünglichen Strahldurchmessers von $1.5 \mathrm{~cm}$ verkleinert und anschließend in einem nichtlinearen Kristall $(\mathrm{BBO}, d=1 \mathrm{~mm}$, $\theta=30.15^{\circ}$ ) mit einer Effizienz von ca. $50 \%$ frequenzverdoppelt, so daß Pulse bei $387.5 \mathrm{~nm}$ mit einer Energie von $0.1 \mathrm{~mJ}$ zur Verfügung stehen. Diese ultravioletten Pulse werden mittels eines dielektrischen Spiegels (HR 390 nm, HT $780 \mathrm{~nm} / 45^{\circ}$ unpol.) vom restlichen, unverdoppelten Licht abgetrennt.

Das ultraviolette Licht, welches im Experiment als Pumpwelle fungiert, passiert eine als Attenuator (Abschwächer) bezeichnete Kombination aus $\lambda / 2$ Verzögerungsplatte und Polarisator (beide AR $400 \mathrm{~nm}$ ), welche es ermöglicht unabhängig voneinander Polarisationszustand und Energie der Pumpwelle einzustellen. Das Licht wird danach mit einer Quarzlinse $(f=100 \mathrm{~mm})$ in eine thermostatierbare Meßküvette (Hellma, 165-QS, Schichtdicke $1 \mathrm{~mm}$ ) fokussiert, wobei die Meßküvette auf einer exzentrisch bewegten Küvettenhalterung befestigt ist, die mit ca. $20 \mathrm{~Hz}$ rotiert, so daß jeder Laserschuß auf zuvor ungepumpte Meßlösung trifft [27]. Auf diese Weise können auch bei geringen Probenvolumina Ausbleich- und/oder Aufheizeffekte vermieden werden. Die Temperatur in der Meßküvette wird mit einem Thermostaten (Julabo, Ultratemp 2000) auf $\pm 0.5^{\circ} \mathrm{C}$ konstant gehalten. Ein möglichst großer Teil der entstehenden, räumlich ungerichteten Fluoreszenz wird mit einem Off-Axis-Parabolspiegel geringer Brennweite und damit großem Öffnungswinkel gesammelt und kollimiert (Janos, Aluminium, 90 - Off-Axis, $f=50.8 \mathrm{~mm})$. Um restliches Pumplicht abzutrennen wird ein Grünglasfilter (Schott, GG420, $1 \mathrm{~mm}$ ) in den Strahlengang der Fluoreszenz gebracht. Anschließend wird die Fluoreszenz über einen Umlenkspiegel geleitet, der mit einem Loch zur Abtrennung verbleibender Pumpstrahlung und gleichzeitigen Einkopplung des Gate-Pulses in den Strahlengang versehen ist. Mittels eines weiteren Parabolspiegels (Janos, Aluminium, 90-Off-Axis, $f=101.6 \mathrm{~mm}$ ) wird die Fluoreszenz in den Aufkonvertierungskristall (BBO, $d=1 \mathrm{~mm}, \theta=34.2^{\circ}$ ) fokussiert. Die Fokussierung erfolgt hierbei mit möglichst großer Brennweite, um innerhalb des Akzeptanzwinkels des nichtlinearen Kristalles zu bleiben und so- 


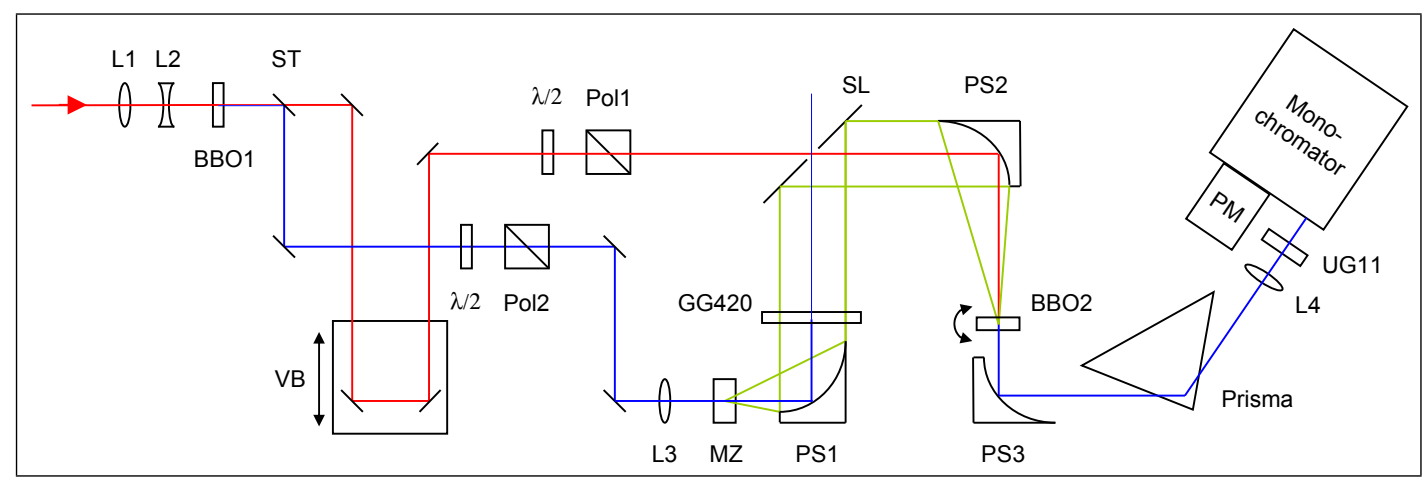

Abbildung 4.6: Schematischer Aufbau des Fluoreszenzkonversionsexperimentes. L1: $f=150 \mathrm{~mm}$, L2: $f=-50 \mathrm{~mm}$, L3: $f=100 \mathrm{~mm}, L 4: f=100 \mathrm{~mm}$, PS1: $f=50.8 \mathrm{~mm}$, PS2: $f=101.6 \mathrm{~mm}$, PS3: $f=76.2 \mathrm{~mm}$, Pol1: Polarisator (AR $800 \mathrm{~nm})$, Pol2: Polarisator (AR $400 \mathrm{~nm})$, BBO1: SHG-Kristall, BBO1: UpConversion-Kristall, ST: Strahlteiler, VB: Verschiebebühne, MZ: Meßzelle, SL: Spiegel mit Loch, PM: Photomultiplier.

mit eine hohe Umwandlungseffizienz zu erreichen (siehe Kapitel 4.4.1). Um die kritische Justage des Fluoreszenzlichtes zu erleichtern, ist es essentiell, die Parabolspiegel auf xyz- bzw. xy-Bühnen (Newport, M460A) zu montieren.

Das restliche, unverdoppelte Laserlicht bei $775 \mathrm{~nm}$ wird als Gatepuls im FUCExperiment verwendet. Es durchläuft zunächst eine variable Verzögerungsstrecke (Physik Instrumente, Verschiebebühne M410-DG; Controller C-844), über welche die Zeitverzögerung zwischen Fluoreszenz und Gatepuls im nichtlinearen Kristall variiert werden kann. Der Gatepuls wird dabei zweimal über die Bühne geleitet, um so den zur Verfügung stehenden Meßbereich auf ca. $1.3 \mathrm{~ns}$ zu verdoppeln (in Abbildung 4.6 nicht dargestellt). Ein weiterer Attenuator ermöglicht auch hier die unabhängige Einstellung von Polarisationszustand und Energie (AR $800 \mathrm{~nm}$ ). Der Gatepuls wird über den Spiegel mit Loch in den Strahlengang der Fluoreszenz kollinear eingekoppelt und mit derselbigen über den zweiten Parabolspiegel in den nichtlinearen Kristall fokussiert, in dem bei geeigneter Winkelstellung nun die Summenfrequenzerzeugung stattfindet. Dazu ist der Kristall auf einer drehbaren Halterung mit Winkelskala befestigt.

Die aus dem Kristall hinaustretende Strahlung wird mit einem weiteren $\mathrm{Pa}$ rabolspiegel (Janos, Aluminium, 90-Off-Axis, $f=76.2 \mathrm{~mm}$ ) parallelisiert und durch ein Prisma zur Abtrennung des restlichen Fluoreszenz- und Gatelichtes geführt (Quarz, $\left.\alpha=68^{\circ}\right)$. Mit einer Quarzlinse $(f=100 \mathrm{~mm})$ wird das aufkonvertierte Licht in einen Monochromator (Beckmann, Brennweite $f=160 \mathrm{~mm}$, Gitter $=600$ Linien $/ \mathrm{mm}, \lambda_{\text {Blaze }}=310 \mathrm{~nm}$, reziproke lineare Dispersion $=11 \mathrm{~nm} / \mathrm{mm}$ ) fokussiert. Vor dessen Eintrittsspalt befindet sich ein Glas- 
filter (Schott, UG11, $1 \mathrm{~mm}$ ) zur Unterdrückung von Streu- und Umgebungslicht. Der Monochromator dient zur weiteren Unterdrückung von Streulicht und desweiteren im Zusammenhang mit der Winkelstellung des nichtlinearen Kristalles zur Frequenzselektion des jeweiligen aufkonvertierten Fluoreszenzlichtes. Die Detektion erfolgt mit einem Photomultiplier (Hamamatsu, 1P28, 9 Dynoden, Betriebsspannung $=1250 \mathrm{~V}$ ) mit elektronischer Verstärkung und Integratorschaltung. Die gesamte Steuerung des Meßablaufes, sowie die Datenaufnahme geschieht von einem Personal-Computer aus, der mit einer AD-Wandlerkarte (Data-Translation, DT 3016, 16 Bit) ausgestattet ist. Das verwendete Computerprogramm für den korrekten Ablauf der Messungen wurde dabei in der Umgebung VEE Onelab (Agilent Technologies) geschrieben.

\subsubsection{Charakterisierung des Meßsystems}

Eine spektrale Kalibration des FUC-Experimentes ist aufgrund der vielen unbestimmbaren Parameter, insbesondere der empfindlichen Abhängigkeit der gemessenen Intensitäten von der jeweiligen Justage, nicht möglich. Es können daher im FUC-Experiment lediglich relative Intensitäten gemessen werden. Die Gewichtung der Fluoreszenzkinetiken kann anhand der stationären Emissionsspektren erfolgen, wenn gewährleistet ist, daß der dynamische Stokes-Shift im betrachteten Zeitfenster abgeschlossen ist. Da dies im untersuchten System nicht der Fall ist, fand eine Gewichtung über zeitaufgelöste Spektren bei einer festen Verzögerungszeit von ca. 500 ps statt, die im TCSPC-Experiment erhalten wurden (siehe Kapitel 5.4.3).

Um das zeitliche Auflösungsvermögen der FUC-Apparatur abzuschätzen, wurde eine Intensitätskreuzkorrelation zwischen Anregungspuls und Gatepuls aufgenommen. Der experimentelle Aufbau mußte zu diesem Zweck nicht verändert werden, da aus dem Anregungspuls resultierendes Streulicht für eine Aufnahme der Kreuzkorrelation über Summenfrequenzerzeugung mit dem Gatepuls bereits ausreichte. Daher ist die gemessene Kurve repräsentativ für den verwendeten experimentellen Aufbau. Eine Gauß-Anpassung der gemessenen Kreuzkorrelation ergibt eine Halbwertsbreite von $\tau_{\mathrm{FWHM}}=300 \mathrm{fs}$ (siehe Abbildung 4.7). Sie kann als ein unteres Limit der Zeitauflösung betrachtet werden. Die tatsächliche Zeitauflösung ist zudem frequenzabhängig, daher ging die Halbwertsbreite $\tau_{\text {FWHM }}$ nicht explizit bei der Anpassung der gemessenen Kinetiken ein, sondern wurde als anpaßbarer Parameter behandelt. Auch der zeitliche Nullpunkt der Kinetiken wurde über die Anpassung bestimmt. Siehe dazu Kapitel 5.4.3. 


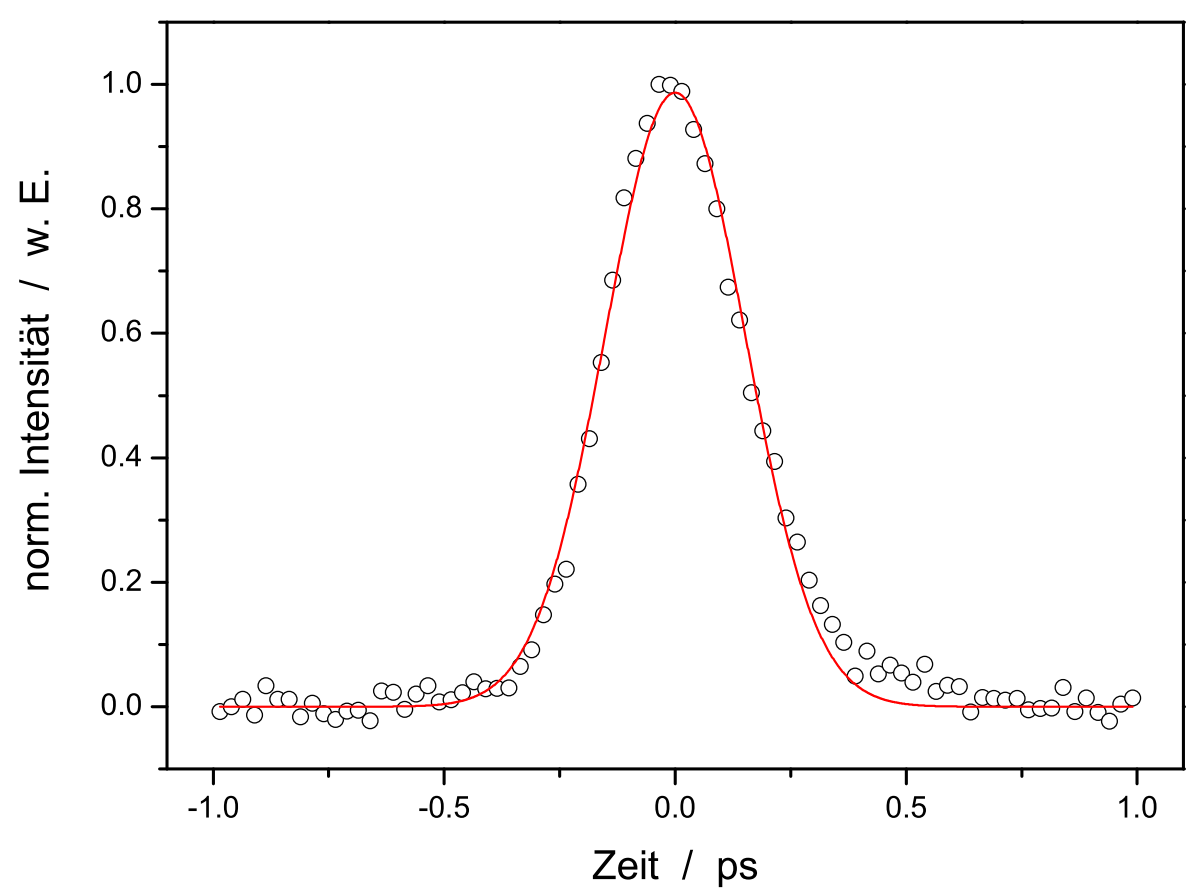

Abbildung 4.7: Intensitätskreuzkorrelation der Anregungspulse und der Gatepulse mit gaußförmiger Anpassung.

\subsubsection{Meßprozedur}

In Ergänzung zu den TCSPC-Messungen zur Untersuchung der Solvatationsdynamik an Lipidmembran/Wasser-Grenzschichten wurden analoge Experimente mit dem FUC-Experiment zur Untersuchung der ultraschnellen Dynamik durchgeführt.

Da das FUC-Experiment aufgrund seines relativ ineffizienten, nichtlinearen Frequenzmischprozesses eine hohe Fluoreszenzintensität benötigt, wurde das System Laurdan/DMPC/Wasser mit einer höheren Farbstoffkonzentration als im TCSPC-Experiment hergestellt $\left(c_{\mathrm{DMPC}}=20 \mathrm{mM}, n_{\mathrm{DMPC}} / n_{\text {Laurdan }}=100: 1\right)$. Um im FUC-Experiment Kinetiken mit einem zufriedenstellenden Signal/RauschVerhältnis zu erhalten, ist es notwendig ausreichend weit entfernt von der Mischfrequenz zwischen Anregungs- und Gatepuls zu detektieren, da in diesem Bereich ein starker Streustrahlungsuntergrund vorliegt, so daß das Signal nicht mehr gegenüber dem Rauschhintergrund diskriminiert werden kann. Daher ist man auf genügend große Fluoreszenz Stokes-Shifts angewiesen, um die Solvatationskorrelationsfunktion mit ausreichender Genauigkeit zu bestimmen. Es konnte daher im FUC-Experiment die Solvatationsdynamik nur innerhalb der flüssig-kristallinen Phase der Membranen bestimmt werden. 
Es wurden Kinetiken im Temperaturbereich von $T=25$ bis $60{ }^{\circ} \mathrm{C}$ im Abstand von $5^{\circ} \mathrm{C}$ aufgenommen. Für jede dieser Temperaturen wurden 17 Kinetiken im Bereich von 268 bis $325 \mathrm{~nm}$ gemessen, entsprechend Fluoreszenzwellenlängen von 410 bis $560 \mathrm{~nm}$ im Abstand von $10 \mathrm{~nm}$. Für jede dieser Kinetiken wurden im Zeitbereich von -100 bis 1000 ps 277 Meßpunkte aufgenommen. Um die relevanten Zeitbereiche zu betonen, wurde mit einem diskontinuierlichem Punktabstand gearbeitet. Im Bereich von -100 bis -6 ps betrug der Punktabstand 2 ps. Um den Anfangsteil der Kinetiken besser bestimmen zu können, wurde von -6 bis 10 ps ein Punktabstand von 0.2 ps gewählt. Im restlichen Teil wurden 150 Punkte mit logarithmisch größer werdendem Punktabstand erfaßt. Jeder Meßpunkt stellt den Mittelwert aus 1000 Einzelschüssen dar, d. h. einer Mittelung über einen Zeitraum von einer Sekunde. Um das Signal/Rausch-Verhältnis weiter zu steigern wurde jede Kinetik $6 \mathrm{Mal}$ gemessen und anschließend gemittelt. Auf diese Weise werden Langzeitschwankungen des gesamten Systems ausgeglichen. Da bei diesem Verfahren eine Einzelmessung ca. 10 Minuten in Anspruch nahm, betrug die Meßzeit für eine Kinetik etwa eine Stunde. Die Spaltbreite des Monochromators betrug $0.5 \mathrm{~mm}$, entsprechend einer spektralen Auflösung $5.5 \mathrm{~nm}$. Die Anregung der Probe erfolgte im magischen Winkel von $54.7^{\circ}$ um Anisotropieeffekte zu vermeiden [158]. Die Anregungsenergie betrug $1-3 \mu \mathrm{J}$, wohingegen der Gatepuls eine Energie von ca. $5 \mu \mathrm{J}$ hatte.

Aufgrund der Lichtempfindlichkeit des Farbstoffes Laurdan, sind die verwendeten Proben einer erheblichen Zersetzung ausgesetzt, was zur Folge hat, daß die Fluoreszenzintensität mit fortschreitender Bestrahlung abnimmt. Daher wurde nach der Messung von jeweils 4-6 verschiedenen Kinetiken, die Meßlösung ausgetauscht. Diese Zersetzungsprozesse und etwaige Zimmertemperaturschwankungen verlangten eine Korrektur des Untergrundes der gemessenen Kinetiken. Dazu wurde vor und nach jeder Einzelmessung die Größe des Signals bei einer negativen Verzögerungszeit, d. h. bei Abwesenheit von Fluoreszenz, gemessen. Im Falle eines Unterschiedes beider Signale wurde der Untergrund linear bezüglich der Zeit interpoliert. Aus den so gewonnen Daten konnte unter zur Hilfenahme von TCSPC-Spektren bei einer festen Verzögerungszeit, für die relative Gewichtung der Intensitäten untereinander, die Solvatationskorrelationsfunktion rekonstruiert werden (siehe Kapitel 5.4.3).

Aufgrund der relativ schwachen Fluoreszenz des verwendeten Farbstoffes Laurdan in den zu untersuchenden Meßlösungen, wurde die Justage des FUCExperimentes mit geeigneten Farbstofflösungen durchgeführt. Dies bietet mehrere Vorteile: Zum einen können solche Lösungen hochkonzentriert hergestellt werden, zum anderen kann mit einer höheren Pumpenergie angeregt werden. Desweiteren kann je nach Wellenlänge ein unterschiedlicher Farbstoff verwendet werden. In dieser Arbeit wurden drei verschiedene Farbstofflösungen zur Justage verwendet: Coumarin 153 und Coumarin 151 in Methanol, sowie Coumarin 4 in alkalisiertem Methanol. Deren stationäre Emissionsspektren sind in Abbildung 4.8 dargestellt. 
Die Struktur auf dem Spektrum des Coumarin 4 resultiert aus verschiedenen emittierenden Spezies in Lösung [211,212]. Nach erfolgter Justage des Setups mit Hilfe dieser Farbstofflösungen, wurde eine identische Zelle in die Küvettenhalterung eingesetzt, welche die zu vermessende Lösung enthielt. Die Küvettenhalterung wurde daher derart konstruiert, daß eine reproduzierbare Positionierung der Meßzellen stattfinden konnte.

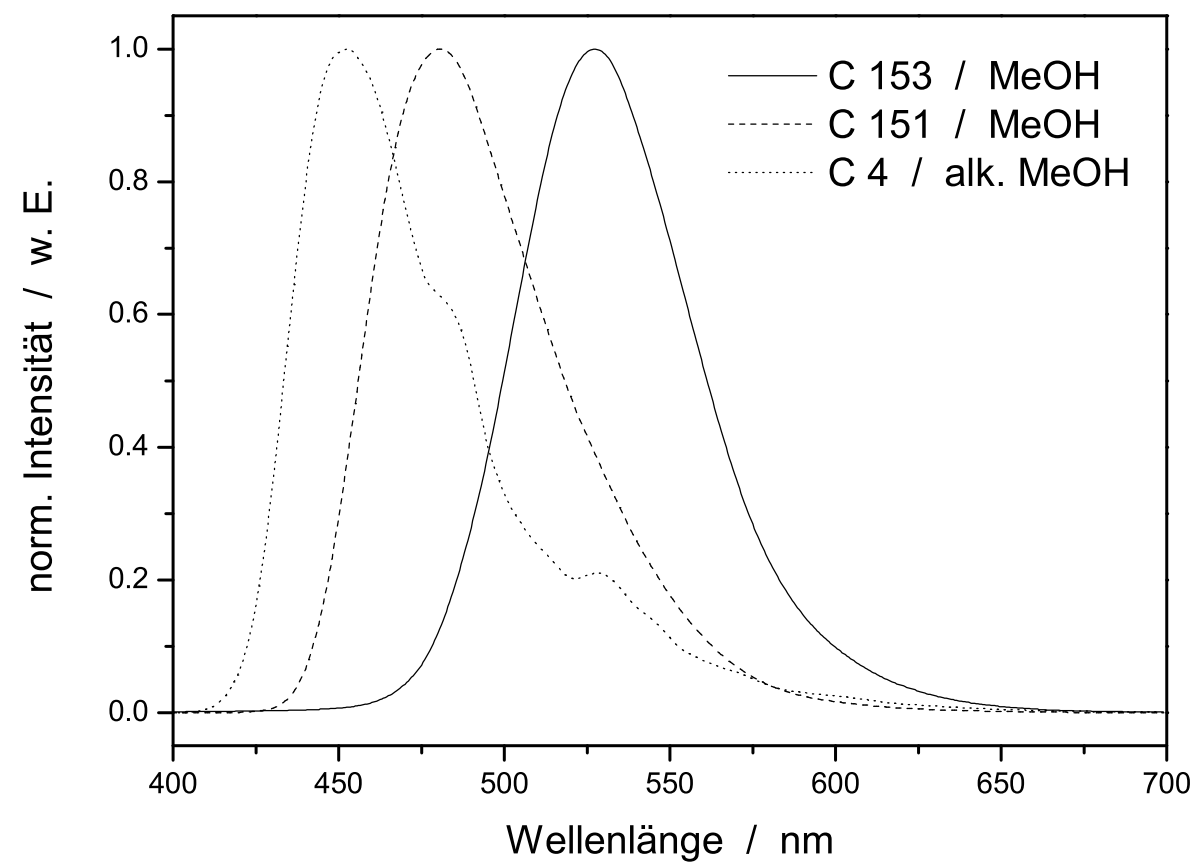

Abbildung 4.8: Fluoreszenzspektren der zur Justage verwendeten Farbstoffe Coumarin 153, Coumarin 151 und Coumarin 4 in Lösung.

\subsection{Photon-Echo-Spektroskopie}

Für die in dieser Arbeit durchgeführten Photon-Echo-Experimente an inversen Mizellen werden ultrakurze, abstimmbare Laserpulse benötigt, um sie den Absorptionsspektren der verwendeten Farbstoffe anpassen zu können. Zur Bereitstellung dieser Pulse wird ein nichtkollinearer optisch-parametrischer Verstärker verwendet, der in Kapitel 4.5.1 kurz vorgestellt wird. Das eigentliche PhotonEcho-Experiment zur Untersuchung der ultraschnellen Solvatationsdynamik in inversen Mizellen wird in Kapitel 4.5.3 behandelt. 


\subsubsection{Nichtkollinearer Optisch-Parametrischer Verstärker}

Zur Erzeugung der abstimmbaren, ultrakurzen Laserpulse für das Photon-EchoExperiment dient ein nichtkollinearer optisch-parametrischer Verstärker (NOPA, Noncollinear Optical Parametric Amplifier), basierend auf den Arbeiten von Riedle und Mitarbeitern [213,214,215].

Bei der optisch-parametrischen Verstärkung handelt es sich um einen DreiWellen-Mischprozeß in einem nichtlinearen Medium, bei dem die sogenannte Pumpwelle $\omega_{\text {Pump }}$ hoher Intensität in eine Signalwelle $\omega_{\text {Signal }}$ und eine Idlerwelle $\omega_{\text {Idler }}$ aufgespalten wird. Wird der Prozeß durch eine sogenannte Seedwelle $\omega_{\text {Seed }}=\omega_{\text {Signal }}$ initiiert, so findet effektiv eine Verstärkung der Seedwelle auf Kosten der Pumpwelle statt. Die Idlerwelle wird dabei in der Regel verworfen. Wird als Seedwelle ein mit einem Chirp behaftetes Weißlichtkontinuum verwendet, so stellt ein optisch-parametrischer Verstärker eine durchstimmbare Laserlichtquelle dar. Für den Drei-Wellen-Mischprozeß gelten Energie- und Impulserhaltung:

$$
\begin{aligned}
& \omega_{\text {Pump }}=\omega_{\text {Signal }}+\omega_{\text {Idler }} \quad \text { bzw } . \\
& \vec{k}_{\text {Pump }}=\vec{k}_{\text {Signal }}+\vec{k}_{\text {Idler }} .
\end{aligned}
$$

Der schematische Aufbau des in dieser Arbeit verwendeten NOPA ist in Abbildung 4.9 dargestellt. Der NOPA wird mit ca. $25 \%$ der Ausgangsleistung des im CPA-2001 erzeugten Laserlichtes bei $775 \mathrm{~nm}$ betrieben. Zunächst wird ein kleiner Teil des Laserlichtes (ca. 4\% Oberflächenreflexion) mit einem Quarzkeil abgetrennt und in einen Saphir-Kristall $(d=2 \mathrm{~mm})$ zur Erzeugung eines Weißlichtkontinuums fokussiert $(f=50 \mathrm{~mm})[216,217,218]$. Das erzeugte Weißlicht wird mit einer weiteren Linse $(f=40 \mathrm{~mm})$ über ein variable Verzögerungsstrecke in den NOPA-Kristall fokussiert (BBO, $d=1 \mathrm{~mm}, \theta=32^{\circ}$ ). Das Weißlichtkontinuum dient im nachfolgenden optisch parametrischen Prozeß als Seedpuls.

Der überwiegende Teil des Laserlichtes wird zunächst mit Hilfe eines GalileiTeleskops um einen Faktor drei bezüglich seines ursprünglichen Strahldurchmessers von $1.5 \mathrm{~cm}$ verkleinert und anschließend in einem Lithiumtriborat-Kristall (LBO, $d=1 \mathrm{~mm}, \theta=29.8^{\circ}$ ) mit einer Effizienz von ca. $40 \%$ frequenzverdoppelt. Die erzeugten Pulse besitzen eine Energie von ca. $85 \mu \mathrm{J}$ bei einer Zentralwellenlänge von $387.5 \mathrm{~nm}$. Sie werden mit einer Quarzlinse $(f=250 \mathrm{~mm})$ unter einem Winkel von $\gamma=6.3^{\circ}$ bezüglich des Weißlichtkontinuums in den NOPA-Kristall weitergeleitet. Der Fokus liegt dabei ca. $10 \mathrm{~cm}$ vor dem Kristall, um die Intensität im Medium unter dessen Zerstörschwelle zu halten. Diese Pulse dienen im optisch-parametrischen Mischprozeß als Pumpwelle. 


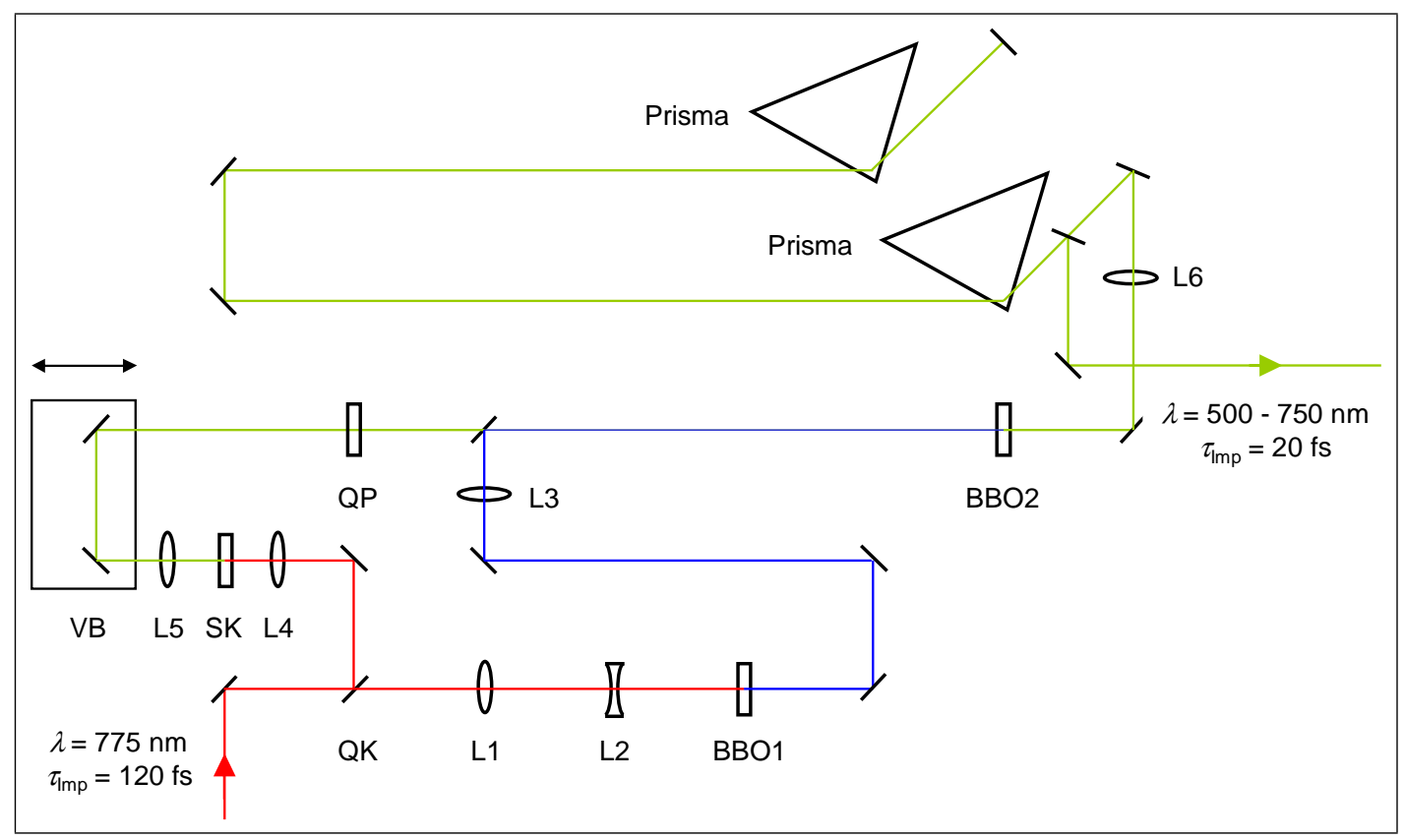

Abbildung 4.9: Schematischer Aufbau des NOPA. L1: $f=150 \mathrm{~mm}$, L2: $f=-50 \mathrm{~mm}, L 3: f=250 \mathrm{~mm}, L 4: f=50 \mathrm{~mm}, L 5: f=40 \mathrm{~mm}, L 6: f=400 \mathrm{~mm}$, BBO1: SHG-Kristall, BBO2: NOPA-Kristall, QK: Quarzkeil, SK: Saphir-Kristall, VB: Verschiebebühne, QP: Quarzplatten.

Bei optimaler räumlicher und zeitlicher Überlagerung der Pump- und Seedpulse im NOPA-Kristall, kann eine effektive Verstärkung des Seedlaserlichtes stattfinden. Im Falle der nichtkollinearen Anordnung kann darüberhinaus bei geeigneter Wahl des Winkels $\gamma$ erreicht werden, daß die Projektion der erzeugten Idlerpulse in Strahlrichtung mit der gleichen Gruppengeschwindigkeit wie die Signalpulse durch den NOPA-Kristall laufen [214]:

$$
v_{\mathrm{g}, \text { Signal }}=\cos (\gamma) \cdot v_{\mathrm{g}, \text { Idler }}
$$

wobei $v_{\mathrm{g}, i}$ die jeweilige Gruppengeschwindigkeit bezeichnet ( $i=$ Signal bzw. Idler). Infolge dieser Anordnung läßt sich die Effizienz des parametrischen Verstärkungsprozesses im Vergleich zur kollinearen Anordnung steigern, während gleichzeitig eine Verlängerung der Pulse unterbunden werden kann.

Da das Weißlichtkontinuum eine deutlich ausgeprägtere Gruppengeschwindigkeitsdispersion (Chirp) im Vergleich zu den Pumppulsen aufweist, werden im parametrischen Verstärkungsprozeß jeweils nur die Frequenzanteile verstärkt, die mit den Pumppulsen gerade zeitlich überlappen. Eine Durchstimmung der SignalWellenlänge kann daher einfach über eine Änderung der Verzögerungsstrecke des 
Weißlichtkontinuums erreicht werden. Die Bandbreite der Pulse läßt sich desweiteren durch Einbringen von Quarzplatten in den Strahlengang des Weißlichtkontinuums in weiten Bereichen variieren, um sie so den gewünschten Anforderungen anzupassen.

Auf diese Weise lassen sich Pulse im Wellenlängenbereich von 500 bis $750 \mathrm{~nm}$ bei Pulsenergien um $10 \mu \mathrm{J}$ erhalten. Die Pulse werden anschließend in einer Prismenkombination (Quarz, $\alpha=68.9^{\circ}$ ) zeitlich komprimiert [219,220], so daß Pulsbreiten im Bereich von $20 \mathrm{fs}$ bei entsprechender Bandbreite über den gesamten Spektralbereich erreicht werden können. Das Spektrum der verwendeten Pulse kann innerhalb der Prismenkombination leicht verändert werden, da hier eine räumliche Aufspaltung in seine einzelnen Frequenzanteile vorliegt. Zur Anpassung der Bandbreite der Pulsspektren an die Absorptionsspektren des verwendeten Farbstoffes wurde daher der Puls nach Bedarf mit Blenden beschnitten. Üblicherweise wurde mit Pulsen einer Impulsdauer um 25 fs, bei einer Bandweite von ca. $35 \mathrm{~nm}$ im verwendeten Wellenlängenbereich um $650 \mathrm{~nm}$ gearbeitet. Das entspricht einem Zeit-Bandweite-Produkt von ca. 0.6, was nicht ganz fourierlimitierten Pulsen entspricht. Eine entsprechende Intensitätsautokorrelationsmessung mit zugehörigem Spektrum bei $668 \mathrm{~nm}$ zeigt Abbildung 4.10. Eine gaußförmige Anpassung der Autokorrelationsfunktion weist eine Halbwertsbreite von $\tau_{\mathrm{FWHM}}=33 \mathrm{fs}$ auf, entsprechend Pulsbreiten von ca. 23 fs.
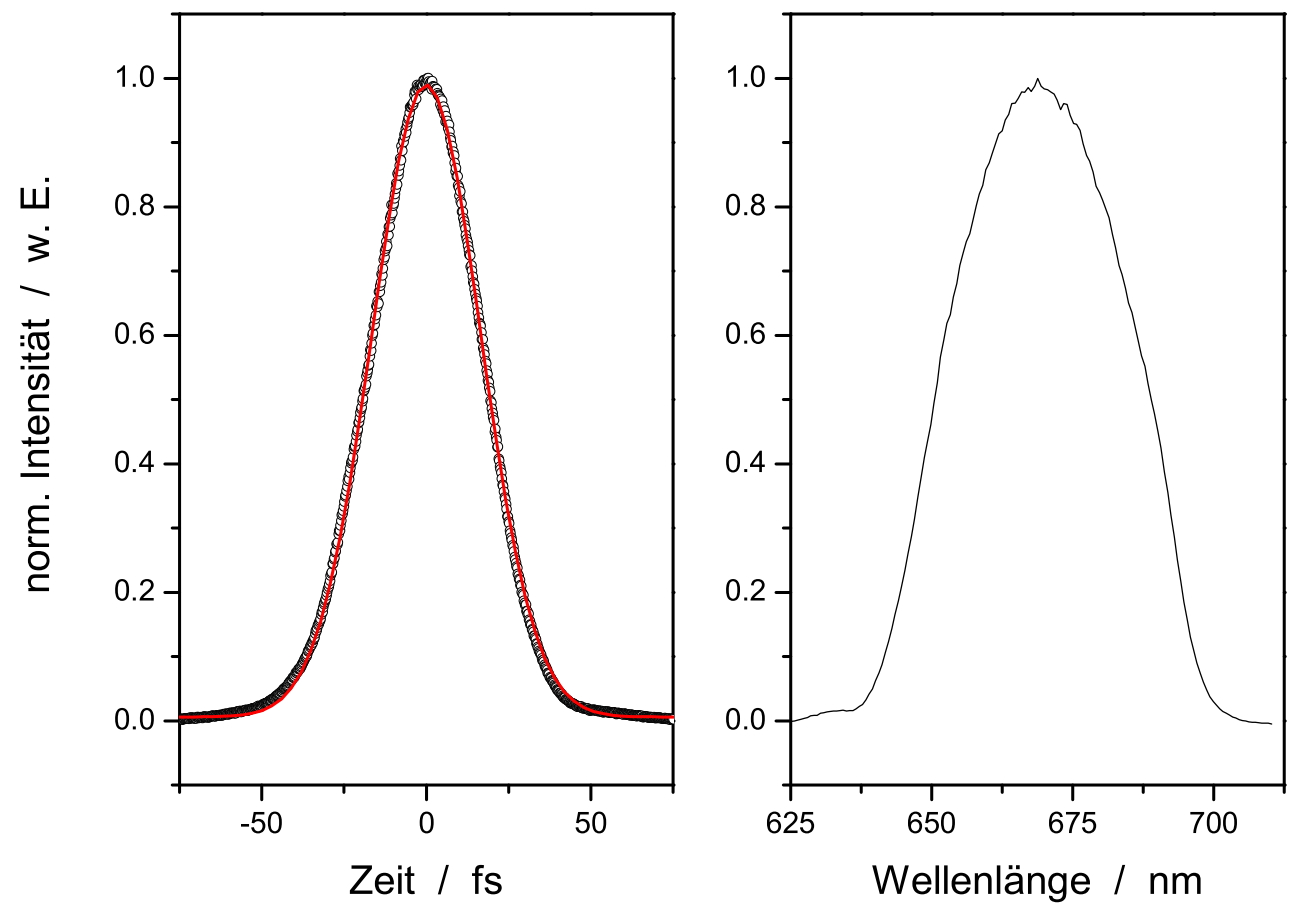

Abbildung 4.10: Intenstitätsautokorrelationsmessung mit gaußförmiger Anpassung und zugehöriges Spektrum des NOPA bei $\lambda=668 \mathrm{~nm}$. 


\subsubsection{Prinzip der Photon-Echo-Spektroskopie}

Die Theorie des Photon-Echo Peakshift Experimentes, sowie die Beziehung des gemessenen Peakshifts zur Solvatationsdynamik wurde in Kapitel 2.3 eingehend beschrieben. Hier sollen nur kurz die wichtigsten Punkte zum Verständnis des experimentellen Ablaufes eines solchen Experimentes wiederholt werden.

Bei einem 3-Puls Photon-Echo Peakshift Experiment (3PEPS) handelt es sich um einen Vier-Wellen-Mischprozeß, wobei das Echo durch die Wechselwirkung dreier Laserpulse $\vec{k}_{1}, \vec{k}_{2}$ und $\vec{k}_{3}$ mit dem verwendeten Chromophor generiert wird. Der erste Puls $\vec{k}_{1}$ erzeugt eine Kohärenz zwischen Grund- und angeregtem Zustand. Der zweite Puls $\vec{k}_{2}$ beendet die Kohärenzperiode $t_{12}$ und startet die Populationsperiode $t_{23}$ (im Grund- oder angeregten Zustand), in der eine spektrale Diffusion stattfindet. Der letzte Puls $\vec{k}_{3}$ beendet auch diese Periode und erzeugt eine zweite Kohärenzperiode. Daraufhin wird das Echosignal $\vec{k}_{S}$ in die Raumrichtung der Phasenanpassung $\vec{k}_{2}+\vec{k}_{3}-\vec{k}_{1}$ entsprechend der Impulserhaltung abgestrahlt. Eine zeitliche Verschiebung des Echo-Maximums bezüglich einer gleichzeitig gemessenen Autokorrelation, die als Nullpunktsreferenz dient, wird als Photon-Echo Peakshift bezeichnet. Ein von Null verschiedener Peakshift beschreibt die Fähigkeit des untersuchten Systems zu rephasieren und kann in Abhängigkeit von der Populationszeit $t_{23}$ mit der Dynamik der Solvatation in Verbindung gebracht werden.

Das Photon-Echo-Experiment besitzt den Nachteil im Vergleich zu Techniken die den zeitabhängigen Fluoreszenz Stokes-Shift messen, daß die Solvatationskorrelationsfunktion nicht direkt beobachtet werden kann. Demgegenüber bietet die Photon-Echo-Technik den Vorteil einer sehr hohen Zeitauflösung, die unterhalb der verwendeten Pulsdauern liegt und prinzipiell nur durch die erreichbare Genauigkeit bei der Bestimmung des Echo-Peakshifts von ca. \pm 1 fs limitiert ist. Zudem ist im Vergleich zu zeitaufgelösten Stokes-Shift Experimenten der Aufwand für die Auswertung der experimentellen Daten als geringer einzuschätzen, da eine zeitintensive Rekonstruktion zeit- und frequenzaufgelöster Spektren entfällt.

Mit dem zur Verfügung stehenden experimentellen Aufbau wurde darüberhinaus die sogenannte Transient-Grating-Spektroskopie (TG) durchgeführt. In diesem Fall wird die Kohärenzperiode $t_{12}=0$ gesetzt, d. h. die beiden Laserpulse $\vec{k}_{1}$ und $\vec{k}_{2}$ werden gleichzeitig in die Probe eingestrahlt und erzeugen dort ein transientes Gitter. Der dritte Puls $\vec{k}_{3}$ wird mit variabler Zeitverzögerung in die Probe eingestrahlt und dort am Gitter entsprechend der Phasenanpassungsbedingung in die Raumrichtung $\vec{k}_{2}+\vec{k}_{3}-\vec{k}_{1}$ gebeugt. Das TG-Signal ist bei ausreichend kurzen Pulsen von kohärenter Wellenpaketdynamik überlagert, so daß diese experimentelle Technik zur Untersuchung der Schwingungsdynamik des verwendeten Chromophors herangezogen werden kann. 


\subsubsection{Experimenteller Aufbau}

Der experimentelle Aufbau des in der vorliegenden Arbeit verwendeten PhotonEcho-Experiments ist in Abbildung 4.11 schematisch dargestellt. Die vom NOPA kommende, abstimmbare Laserstrahlung wird mittels sechs Strahlteilerwürfel (Linos Photonics, $50 \%$ Reflexion, AR $800 \mathrm{~nm}$ ) derart aufgeteilt, daß sich sieben Laserstrahlen gleicher Intensität und Dispersion ergeben. Da die Intensitäten der Laserstrahlen in einem Photon-Echo-Experiment sehr gering gehalten werden müssen (ca. 5 nJ pro Puls), um störende Effekte zu vermeiden die bei höheren Intensitäten auftreten können, wurde die aus dem NOPA kommende Strahlung mit Neutralglasfiltern entsprechend abgeschwächt [36].

Im sogenannten Meßzweig dienen drei dieser Strahlen (Strahl 1-3 in der Abbildung 4.11) zur Erzeugung des 3-Puls-Echos entsprechend obiger Beschreibung. Die Strahlen $1\left(\vec{k}_{1}\right)$ und $2\left(\vec{k}_{2}\right)$ werden dabei über variable Verzögerungsstrecken (Melles Griot, Nanomover; Controller Nanomotion II) zur unabhängigen Einstellung der Zeiten $t_{12}$ und $t_{23}$ geleitet, während Strahl $3\left(\vec{k}_{3}\right)$ eine feste Verzögerungsstrecke durchläuft. Durch doppelte Ausnutzung der Verzögerungsstrecken (in der Abbildung nicht gezeigt) bieten die Translationsbühnen einen zeitlichen Meßbereich von ca. 330 ps. Die drei Strahlen werden in einem Winkel von ca. $0.7^{\circ}$ mittels

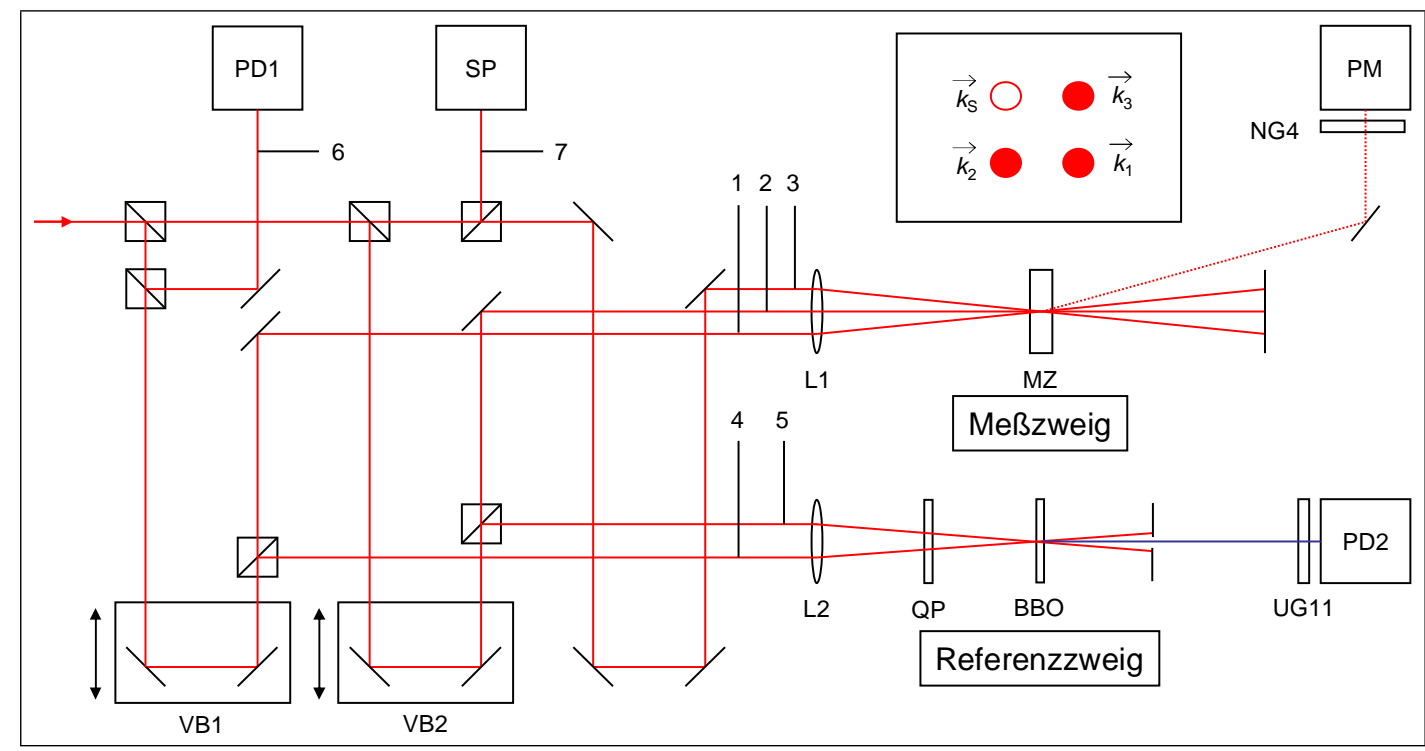

Abbildung 4.11: Schematischer Aufbau des Photon-Echo-Experimentes. L1: $f=300 \mathrm{~mm}$, L2: $f=300 \mathrm{~mm}$, VB1: Verschiebebühne 1, VB2: Verschiebebühne 2, MZ: Meßzelle, QP: Quarzplatte, BBO: Autokorrelationskristall, PD1: Photodiode, PD2: Photodiode, PM: Photomultiplier, SP: Spektrometer, 1-7: Numerierung der Strahlen, Inlet: Einstrahlgeometrie der Strahlen 1-3 in die Meßzelle. 
einer Linse $(f=300 \mathrm{~mm})$ in die thermostatierbare Meßzelle (Hellma, 165-QS, Schichtdicke $1 \mathrm{~mm}$ ) fokussiert. Die Meßzelle ist analog dem Fluoreszenzaufkonvertierungsexperiment auf einer exzentrisch bewegten Zellhalterung montiert, um Ausbleich- und Aufheizeffekte zu vermeiden. Die Temperatur in der Meßzelle kann mittels eines Thermostaten (Lauda, RC 6) auf $\pm 0.5^{\circ} \mathrm{C}$ konstant gehalten werden. Die drei Strahlen werden in der sogenannten Boxcar-Geometrie in die Meßzelle fokussiert, d. h. die Wellenvektoren zeigen in drei Ecken eines Quadrates. Das auf diese Weise gemäß den Phasenanpassungsbedingungen in der vierten Ecke entstehende Echo (siehe Inlet in Abbildung 4.11) ist damit räumlich separiert und wird mittels einer Blende und einem Glasfilter (Schott, NG 4, $1 \mathrm{~mm}$ ) von divergentem Streu- und Umgebungslicht bereinigt. Ein Photomultiplier (Hamamatsu, R928, 7 Dynoden, Betriebsspannung $=700$ V) mit elektronischer Verstärkung und Integratorschaltung dient als Detektor.

Da das erzeugte Photon-Echo integral detektiert wird, ist die Information über den interessierenden Peakshift bei festen Zeiten $t_{12}$ und $t_{23}$ zunächst verloren. Um dennoch an diese Information zu gelangen, bedient man sich eines einfachen Tricks. Beide Bühnen werden zu einer festen Verzögerungszeit $t_{23}$ gefahren. Dort wird die Verzögerungszeit $t_{12}$ in einem kleinen Zeitintervall variiert. Infolgedessen repräsentiert das dabei erzeugte Photon-Echo-Signal auch bei integraler Detektion in Abhängigkeit der Verzögerung $t_{12}$ den zeitlichen Verlauf des Echos. Die zeitliche Lage des Maximums des detektierten Signals bezüglich einer Nullpunktsreferenz gibt dann den gesuchten Peakshift an. Eine andere Methode, welche den zeitlichen Verlauf der erzeugten Echos direkt untersucht, ist das sogenannte Time-Gated-Photon-Echo. Hierbei wird der zeitliche Verlauf des Echos durch Up-Conversion-Techniken, wie sie in Kapitel 4.4 vorgestellt wurden, untersucht $[35,36]$.

Die Strahlen 4 und 5 dienen zur Bestimmung der Autokorrelation als Nullpunktsreferenz (Referenzzweig), da in der Boxcargeometrie im Vergleich zu anderen Einstrahlgeometrien (z. B. Dreiecksgeometrie) lediglich ein einzelnes Echosignal detektiert wird und somit nur eine relative Bestimmung des Peakshifts erfolgen kann. Beide Strahlen durchlaufen die gleiche Verzögerungsstrecke wie die Strahlen 1 bzw. 2 , so daß die simultan gemessene Autokorrelation stets eine zeitliche Referenz zum gemessen Echo darstellt. Auf diese Weise kann das Signal/RauschVerhältnis erheblich verbessert werden, da zeitliche Schwankungen des Lasers und damit des Nullpunktes ausgeglichen werden können. Die Messung liefert aber dennoch keine Absolutwerte des Peakshifts, weil es bei der Einstellung der Zeitnullpunkte unvermeidlich zu einem zeitlichen Unterschied zwischen den Strahlen 1, 2 und 3 und den Strahlen 4 und 5 kommen kann (siehe Kapitel 4.5.4). Der in der Boxcar-Geometrie bestimmte Peakshift ist daher mit einem konstanten Offset im Bereich weniger Femtosekunden behaftet. Zum Ausgleich der im Meßzweig erhöhten Dispersion durch die Meßzelle durchlaufen die Strahlen 4 und 5 zunächst eine planparallele Quarzplatte (Schichtdicke $=2 \mathrm{~mm}$ ). Danach werden sie mit 
einer Linse $(f=300 \mathrm{~mm})$ unter einem Winkel von ca. $0.7^{\circ}$ in einen nichtlinearen Kristall (BBO, $d=0.1 \mathrm{~mm}, \theta=40.5^{\circ}$ ) zur Erzeugung der zweiten Harmonischen fokussiert. Die frequenzverdoppelte Strahlung wird mit einer Blende und einem Glasfilter (Schott, UG11, $1 \mathrm{~mm}$ ) von Streu- und Umgebungslicht befreit und mit einer Photodiode (Hamamatsu, S1336-8BQ) registriert.

Die beiden verbliebenen Strahlen 6 und 7 dienen zur ständigen Analyse der vom NOPA kommenden Laserstrahlung bezüglich Energie und Spektrum. Die zur Verfügung stehenden Pulsenergien werden mit einer kalibrierten Photodiode überwacht, während das Spektrum mit einem Spektrographen (Bausch \& Lomb, Brennweite $f=100 \mathrm{~mm}$, Gitter $=1350$ Linien $/ \mathrm{mm}$ ) und einer Photodiodenzeile (Hamamatsu, S3901) ständig registriert wird.

Die gesamte Steuerung des Meßablaufs, insbesondere die Ansteuerung der Translationsbühnen, sowie die Datenaufnahme sowohl der 3-Puls Photon-Echo Peakshift Messungen als auch der TG-Messungen geschieht von einem PersonalComputer aus, der mit einer AD-Wandlerkarte (Data-Translation, DT 3016, 16 Bit) ausgestattet ist. Die verwendeten Computerprogramme für den korrekten Ablauf der Messungen wurde dabei in der Umgebung VEE Onelab (Agilent Technologies) geschrieben.

\subsubsection{Meßprozedur}

Zur Bestimmung der Solvatationsdynamik von eingeschränktem Wasser in inversen Mizellen wurden 3-Puls Photon-Echo Peakshift Messungen am System Isooktan/AOT/Wasser/HIDCI durchgeführt. Der Grad der Einschränkung des Wassers läßt sich dabei leicht über die Größe des Wassereinschlusses in den inversen Mizellen einstellen. Der Durchmesser des Wassernanotröpfchens ist proportional zur zentralen Größe $\mu$, welche als Verhältnis der Konzentration von Wasser zur Konzentration der grenzflächenbildenden Substanz AOT in der Meßlösung definiert ist:

$$
\mu=\frac{\left[\mathrm{H}_{2} \mathrm{O}\right]}{[\mathrm{AOT}]} .
$$

Es wurden sechs verschieden Lösungen mit $\mu=1,2,4,8,16$ und 32 untersucht. Als Farbstoffsonde kam der Farbstoff HIDCI zur Anwendung, welcher die Eigenschaften hat, sich im Wassernanotröpfchen der inversen Mizellen zu lösen und so selektiv auf die Dynamik des Wassers zu sein. Die optischen Dichten der untersuchten Lösungen wurden auf $O D=0.5$ für die größeren Mizellen eingestellt, wohingegen für $\mu=2$ eine optische Dichte von 0.2 und für $\mu=1$ optische Dichte von 0.1 gewählt werden mußte. Eine höhere optische Dichte ist für diese Mizellengrößen nicht realisierbar, weil der geringe Wassergehalt solcher Mizellen nur eine gewisse Farbstoffmenge zuläßt. Um die Dynamiken innerhalb des Wassernanotröpfchens inverser Mizellen einordnen zu können, wurde desweite- 
ren die Dynamik des verwendeten Farbstoffes HIDCI in reinem Wasser bei einer optischen Dichte von $O D=0.7$ untersucht. Alle Meßlösungen wurden auf eine Temperatur von $25^{\circ} \mathrm{C}$ thermostabilisiert. Die verwendete Laserstrahlung wurde so eingestellt, daß deren Zentralfrequenz mit dem 0-0-Übergang des Farbstoffes im untersuchten System zusammenfiel $(\lambda=665 \mathrm{~nm}$ in den inversen Mizellen, $\lambda=650 \mathrm{~nm}$ in reinem Wasser). Das Spektrum wurde auf eine Bandbreite von ca. $35 \mathrm{~nm}$ eingestellt, woraus sich Pulsbreiten um 25 fs ergaben.

Die exakte Einstellung des Nullpunktes und die Überlagerung der Strahlen in der Meßzelle ist für die durchgeführten Experimente von grundlegender Wichtigkeit. Dazu wurde die Meßzelle zunächst durch einen nichtlinearen Kristall ersetzt $\left(\mathrm{BBO}, d=0.1 \mathrm{~mm}, \theta=40.5^{\circ}\right)$ und die zwischen den drei eingestrahlten Laserpulsen entstehenden zweiten Harmonischen zur Justage verwendet. Durch Optimierung der erzeugten SHG-Intensitäten konnte sowohl eine exakte Überlagerung aller drei Strahlen, sowie deren zeitlicher Nullpunkt untereinander als auch mit der simultan gemessenen Autokorrelation eingestellt werden. Der Austausch des Kristalls durch die Meßzelle führt zu einer zeitlichen Verschiebung des Nullpunktes zwischen Meßzweig und Referenzzweig, was aber weitestgehend durch die zusätzliche Quarzplatte im Referenzzweig ausgeglichen werden kann. Dennoch führt der Austausch dazu, daß der Peakshift bei dieser Methode nur auf einen gewissen Offset von wenigen Femtosekunden genau bestimmt werden kann.

Die Aufnahme der Peakshifts in Abhängigkeit der Verzögerungszeiten $t_{23}$ repräsentiert die gesuchte Solvatationsdynamik. Die Bestimmung des Peakshifts zu einer festen Zeit $t_{23}$ geschieht nach oben beschriebener Prozedur. Die Bühne 1 wird dabei im sogenannten Rapid-Scan-Betrieb zur Durchstimmung der Verzögerungszeiten $t_{12}$ im Bereich von -150 fs bis +150 fs eingesetzt [186], was bei einer Repetitionsrate von $1 \mathrm{kHz}$ und einer Bühnengeschwindigkeit von $0.15 \mathrm{~mm} / \mathrm{s}$ etwa 1200 Meßpunkten pro Echo- und Autokorrelationssignal entspricht. Jedes Echo, sowie die zugehörige Autokorrelationsmessung, wurden zur Steigerung des Signal/Rausch-Verhältnisses $10 \mathrm{Mal}$ (ab $t_{23}=20 \mathrm{ps}$ aufgrund der reduzierten Echointensität $15 \mathrm{Mal}$ ) gemessen und automatisch gemittelt. Nach erfolgter Messung eines Echos, fuhren die Bühnen im konventionellen Step-Scan-Modus die nächste Verzögerungszeit $t_{23}$ an. Ein typisches Signal in Abhängigkeit der Verzögerungszeit $t_{12}$ für konstantes $t_{23}$ zeigt Abbildung 4.12. Solche Signale wurden an 360 verschiedenen Verzögerungszeiten $t_{23}$ im Bereich von -20 fs bis 300 ps bestimmt, wobei eine diskontinuierliche Verteilung dieser Zeiten gewählt wurde, um die relevanten Zeitbereiche der zugrundeliegenden Dynamiken zu betonen. Von $-20 \mathrm{fs}$ bis $200 \mathrm{fs}$ betrug der Punktabstand $2 \mathrm{fs}$, um den Inertialteil der Dynamik aufzulösen. Von $200 \mathrm{fs}$ bis 2 ps wurden die Echos im Abstand von 12 fs detektiert. Von 2 ps bis 300 ps wurden insgesamt 100 Echosignale mit logarithmisch größer werdendem Punktabstand aufgenommen. Die Aufnahme einer einzelnen Peakshiftmessung in Abhängigkeit von $t_{23}$ dauert ca. 8 Stunden und wurde in der 


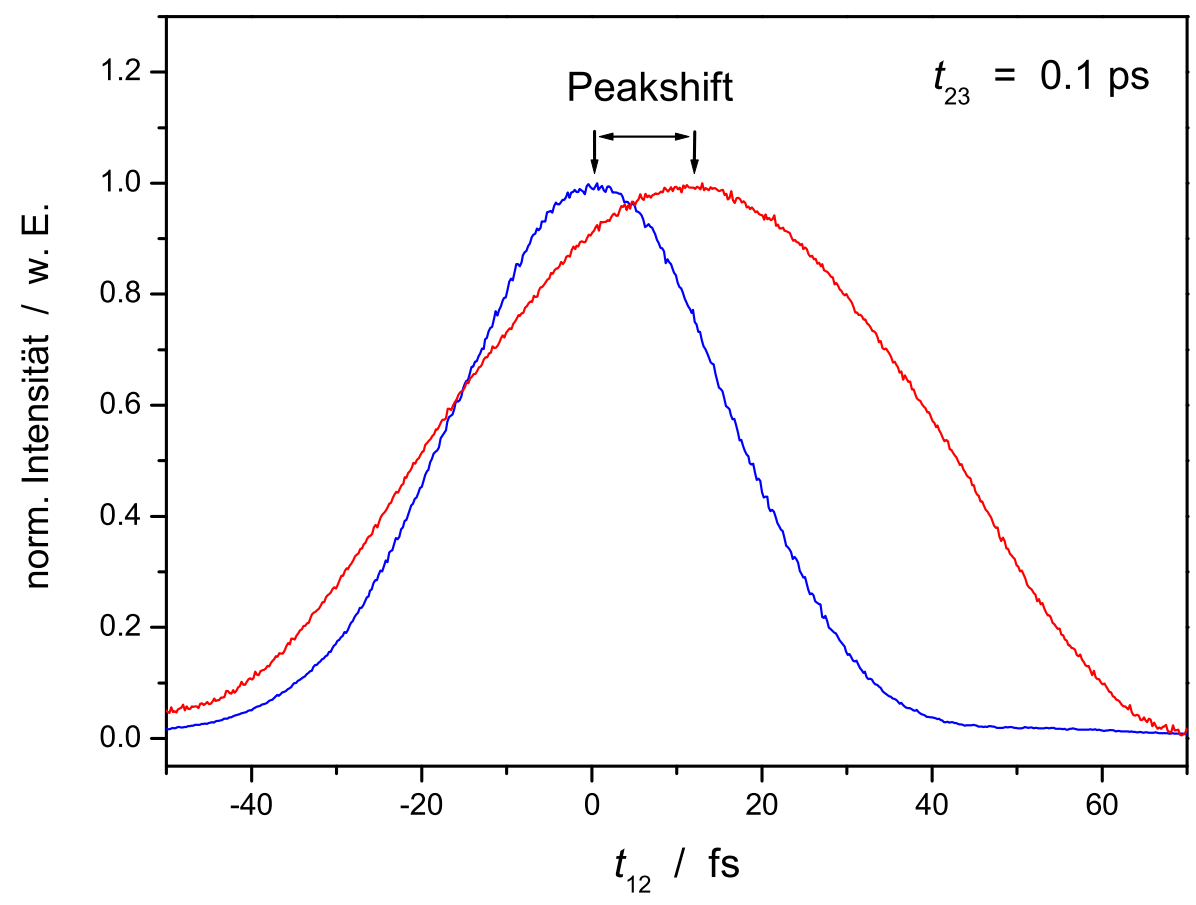

Abbildung 4.12: Typisches Meßsignal im 3PEPS-Experiment. Blau: Autokorrelation; Rot: Echosignal.

Regel über Nacht durchgeführt, um stabile Temperaturbedingungen über diesen Zeitraum zu gewährleisten.

Die Bestimmung des Peakshifts erfolgte mit einem von H. Bürsing [27] geschriebenen Computerprogramm in der Rechenumgebung Matlab (The MathWorks Inc., Version 6.0.0.88). Die gemessenen Echo- und Autokorrelationsrohdaten wurden zunächst mathematisch geglättet, indem jedem Datenpunkt der Mittelwert der Intensität aus den 15 am nächsten liegenden Punkten auf jeder Seite zugeordnet wurde. Anschließend wurden die Signale auf eins normiert und im weiteren nur die oberen $20 \%$ der Signale betrachtet. An die so erhaltenen Daten wurden Polynome dritten Grades angepaßt und deren Maxima bestimmt. Die Differenz beider Maxima ergab den sogenannten Echo-Peakshift, welcher gegen $t_{23}$ aufgetragen die zugrundeliegenden Dynamiken der Solvatation beschreibt (siehe Kapitel 6.2 und Kapitel 6.3).

Zur Untersuchung der Schwingungsdynamik des verwendeten Chromophors HIDCI wurden darüberhinaus Transient-Grating-Messungen der hergestellten Probenlösungen durchgeführt. Im Vergleich zu den Photon-Echo Peakshift Messungen ändert sich lediglich die Ansteuerung der Bühnen. Die Verzögerungszeit $t_{12}$ wurde auf Null gesetzt und nicht variiert, während die zweite Bühne im Rapid- 
$S c a n$-Betrieb die Verzögerungszeit $t_{23}$ in einem Bereich von -1 ps bis 7 ps durchfuhr. Bei einer Bühnengeschwindigkeit von $1.2 \mathrm{~mm} / \mathrm{s}$ und einer Repetitionsrate von $1 \mathrm{kHz}$ wurden dabei insgesamt 4000 Punkte aufgenommen. Eine Messung wurde üblicherweise über 500 Einzelmessungen gemittelt, was ca. 1.5 Stunden in Anspruch nahm. Die erhaltenen Transient-Grating-Signale weisen periodisch oszillierende Signalbeiträge auf, welche kohärenter Wellenpaketdynamik zuzuschreiben sind. Durch geeignete Analyse kann daraus die Schwingungsdynamik des verwendeten Chromophors in seiner jeweiligen Umgebung bestimmt werden (siehe Kapitel 6.4).

\subsection{Verwendete Chemikalien und Probenpräpa- ration}

Zur Herstellung der untersuchten Probenlösungen wurden ausschließlich kommerziell erhältliche Chemikalien verwendet, welche ohne weitere Reinigung verwendet wurden. Lediglich das AOT mußte vor Verwendung nach unten beschriebener Prozedur getrocknet werden, da es sich um einen stark hygroskopischen Stoff handelt.

In den folgenden Tabellen sind die in dieser Arbeit verwendeten, relevanten Chemikalien mit Herstellerangabe und Reinheitsgrad angegeben (soweit bekannt). Dabei faßt Tabelle 4.1 die zur Herstellung der Vesikellösungen verwendeten Chemikalien zusammen, während Tabelle 4.2 die Chemikalien zur Präparation der inversen Mizellen enthält. Die Verfahren zur Probenpräparation der Lipid-Vesikel und der inversen Mizellen werden in den folgenden Unterkapiteln 4.6.1 bzw. 4.6.2 angegeben.

\begin{tabular}{|c|c|c|c|}
\hline Chemische Bezeichnung & $\begin{array}{c}\text { Kurz- } \\
\text { bezeichnung }\end{array}$ & Hersteller & Reinheit \\
\hline $\begin{array}{c}\text { 6-Lauroyl- } \\
\text { 1,2-Dimethylaminonaphthalin } \\
\text { 3-phosphatidylcholin }\end{array}$ & Laurdan & $\begin{array}{c}\text { Molecular } \\
\text { Probes }\end{array}$ & k. A. \\
- & WMPC & Fluka & $\geq 99 \%$ \\
\hline
\end{tabular}

Tabelle 4.1: Zur Herstellung der Vesikel verwendete Chemikalien. 


\begin{tabular}{|c|c|c|c|}
\hline Chemische Bezeichnung & $\begin{array}{c}\text { Kurz- } \\
\text { bezeichnung }\end{array}$ & Hersteller & Reinheit \\
\hline $\begin{array}{l}\text { Hexamethylindo- } \\
\text { dicarbocyaniniodid }\end{array}$ & HIDCI & $\begin{array}{l}\text { Lambda } \\
\text { Physik }\end{array}$ & k. A. \\
\hline $\begin{array}{l}\text { Natrium Bis(2-ethylhexl)- } \\
\text { sulfobernsteinsäure }\end{array}$ & AOT & $\begin{array}{l}\text { Sigma- } \\
\text { Aldrich }\end{array}$ & $98 \%$ \\
\hline 2,2,4-Trimethylpentan & Isooktan & Merck & $\geq 99.5 \%$ \\
\hline- & Wasser & - & 2-fach destilliert \\
\hline
\end{tabular}

Tabelle 4.2: Zur Herstellung der inversen Mizellen verwendete Chemikalien.

\subsubsection{Präparation der Lipid-Vesikel}

Zur Untersuchung der Solvatationsdynamik von Wasser an Lipidmembran/Wasser-Grenzschichten wurden unilamellare Vesikel hergestellt (siehe Kapitel 5). Für die unterschiedlichen experimentellen Verfahren wurden Lösungen mit einer Lipidkonzentration von $c_{\mathrm{DMPC}}=10 \mathrm{mM}$ und $20 \mathrm{mM}$ präpariert. Das Verhältnis von Lipid zu Farbstoff $n_{\text {DMPC }} / n_{\text {Laurdan }}$ betrug $1000: 1$ bzw. $100: 1$.

Die Herstellung der Vesikel erfolgte prinzipiell nach in der Literatur beschriebenen Standardmethoden [221]. Die benötigte Menge an Lipid wurde abgewogen und in einer entsprechenden Menge einer Dichlormethanlösung des Farbstoffes Laurdan bekannter Konzentration gelöst. Das Lösungsmittel wurde anschließend im Stickstoffstrom abgezogen. Um das Dichlormethan vollständig zu entfernen, wurde die Substanz über Nacht im Ölpumpenvakuum aufbewahrt. Anschließend wurde der verbleibende Rückstand in einer entsprechenden Menge Wasser aufgenommen und bis zum Erreichen einer homogenen Dispersion gerührt. Die so erzeugten multilamellaren Vesikel wurden durch Extrudieren in unilamellare Vesikel überführt. Extrudieren bezeichnet das wiederholte Hindurchpressen multilamellarer Vesikellösungen durch Membranfilter einer bestimmten Porengröße. Dadurch werden unilamellare Vesikel erhalten, deren Größenverteilung durch die Porengröße der verwendeten Membranfilter bestimmt ist. Es wurde ein Extruder der Firma Avestin, Inc. (Liposo-Fast) verwendet. Weiterhin kamen Polycarbonatfilter der Firma Avestin, Inc. mit einer Porengröße von $100 \mathrm{~nm}$ zur Anwendung. Die resultierende Größenverteilung der Vesikel ist schmal und bei ca. $80 \mathrm{~nm}$ zentriert [221]. Um eventuell gelöste Luft aus den Vesikellösungen zu entfernen wurde die extrudierte Lösung für ca. 15 Minuten im Wasserstrahlpumpenvakuum aufbewahrt. 
Da die auf diese Weise erzeugten unilamellaren Vesikel nur eine begrenzte Lebensdauer haben und sich innerhalb weniger Tage wieder multilamellare Vesikel bilden, wurden stets frisch extrudierte Lösungen untersucht. Unextrudierte Lösungen wurden generell im Kühlschrank bei ca. $8^{\circ} \mathrm{C}$ aufbewahrt. Aufgrund der Lichtempfindlichkeit des verwendeten Farbstoffes Laurdan wurde bei der Probenpräparation und Aufbewahrung darauf geachtet, den Probenlösungen möglichst wenig Licht auszusetzen.

\subsubsection{Präparation der Inversen Mizellen}

Zur Untersuchung der Solvatationsdynamik des in inversen Mizellen eingeschlossenen Wassers wurden Lösungen unterschiedlicher Größen mit $\mu=1,2,4,8,16$ und 32 nach Gleichung 4.24 hergestellt. Der zweite Parameter stellt die gewählte optische Dichte von $O D=0.5$ für die größeren Mizellen, sowie $O D=0.2$ für $\mu=2$ und $O D=0.1$ für $\mu=1$ dar.

Da es sich bei dem Detergenz AOT um eine stark hygroskopische Substanz handelt, mußte es vor Verwendung getrocknet werden. Dazu wurden ca. 10 Gramm AOT in so viel heißem Methanol aufgenommen, daß die Substanz gerade gelöst wurde. Das Lösungsmittel wurde daraufhin im Vakuum abgezogen. Zurück blieb eine klebrige Masse, die im heißen Stickstoffstrom einen Schaum bildet. Der Stickstoffstrom wurde so lange fortgesetzt, bis der Schaum trocken war. Die verbliebene Substanz wurde zerkleinert und unter Rühren einige Stunden im Vakuum aufbewahrt. Das auf diese Weise getrocknete AOT wurde bis zur Verwendung unter Luftausschluß aufbewahrt.

Die Präparation der Lösungen erfolgte nach Standardmethoden. Dazu wurde zunächst eine entsprechende Menge an getrocknetem AOT eingewogen und in einem entsprechenden Volumen Isooktan gelöst. Daraufhin wurde ein entsprechendes Volumen einer HIDCI/Wasser-Lösung bekannter Konzentration hinzugegeben und kräftig geschüttelt. Die eingewogene Masse des AOT und die Volumina der flüssigen Komponenten wurde dabei derart gewählt, daß Lösungen mit entsprechendem $\mu$ und optischer Dichte $O D$ erhalten wurden (siehe Tabelle 6.1). Um Verdunstungseffekte zu vermeiden, wurden die Lösungen bei ca. $8^{\circ} \mathrm{C}$ im Kühlschrank aufbewahrt. 


\section{Kapitel 5}

\section{Lipid-Vesikel}

Im folgenden Kapitel werden die am Modellsystem der Lipidmembran/WasserGrenzschicht erhaltenen Meßdaten, sowie deren Auswertung vorgestellt. Die erworbenen Erkenntnisse werden eingehend diskutiert. Von Interesse ist dabei insbesondere das Studium der Solvatationsdynamik in Abhängigkeit der in der Membran auftretenden Phasenzustände. Als Sonde für die Solvatation wird dabei der Farbstoff Laurdan verwendet, welcher über eine lange Alkylkette nichtkovalent in der DMPC-Lipidmembran verankert ist und dessen Chromophor an der Lipidmembran/Wasser-Grenzschicht lokalisiert ist.

Zunächst werden in Kapitel 5.1 Ergebnisse zur Charakterisierung der spektroskopischen Eigenschaften des Farbstoffes Laurdan außerhalb einer Membranumgebung vorgestellt. Kapitel 5.2 wendet sich den Lipidmembran/WasserGrenzschichten zu. Während zunächst der Polymorphismus des betrachteten Systems mit kalorimetrischen Methoden untersucht wird, widmet sich das anschließende Unterkapitel seinen stationären spektroskopischen Eigenschaften. In Kapitel 5.3 werden die Anisotropieeigenschaften des Farbstoffes in der Lipidmembran anhand des Wobble-in-Cone-Modells beschrieben. Danach folgt die ausführliche Beschreibung der Solvatationsdynamik an der Lipidmembran/WasserGrenzschicht, welche mit den zeitaufgelösten Methoden des zeitkorrelierten Einzelphotonenzählens und der Fluoreszenzaufkonvertierung bestimmt wurde (siehe Kapitel 5.4). Die Ergebnisse werden in Kapitel 5.5 eingehend diskutiert, während Kapitel 5.6 die erhaltenen Erkenntnisse knapp zusammenfaßt. 


\subsection{Untersuchungen an Laurdan}

Um zunächst den Farbstoff Laurdan außerhalb einer Membranumgebung zu charakterisieren, wurden stationäre Spektren in den Lösungsmitteln $n$-Hexan, Ethanol und Methanol aufgenommen. Die Absorptions-, Anregungs- und Emissionsspektren des Laurdans im jeweiligen Lösungsmittel bei $T=25^{\circ} \mathrm{C}$ sind in Abbildung 5.1 dargestellt. Die Aufnahmeparameter der mit dem Fluoreszenzspektrometer Fluorolog 3 der Firma Jobin Yvon registrierten Spektren sind in Tabelle 5.1 zusammengefaßt.

\begin{tabular}{|c|c|c|c|c|}
\hline $\begin{array}{c}\text { Lösungs- } \\
\text { mittel }\end{array}$ & Konzentration & $\begin{array}{c}\text { Detektions- } \\
\text { art }\end{array}$ & $\begin{array}{c}\text { Emission } \\
\boldsymbol{\lambda}_{\mathbf{E x c}}\end{array}$ & $\begin{array}{c}\text { Anregung } \\
\boldsymbol{\lambda}_{\text {Det }}\end{array}$ \\
\hline$n$-Hexan & $10 \mu \mathrm{M}$ & $\mathrm{FF}$ & $340.0 \mathrm{~nm}$ & $420 \mathrm{~nm}$ \\
Ethanol & $22 \mu \mathrm{M}$ & $\mathrm{FF}$ & $387.5 \mathrm{~nm}$ & $485 \mathrm{~nm}$ \\
Methanol & $4.6 \mu \mathrm{M}$ & $\mathrm{FF}$ & $387.5 \mathrm{~nm}$ & $500 \mathrm{~nm}$ \\
\hline
\end{tabular}

Tabelle 5.1: Zusammenfassung der Aufnahmeparameter der stationären Spektren von Laurdan in n-Hexan, Methanol und Ethanol.

Eine Messung von Laurdan in reinem Wasser wäre insbesondere im Vergleich zu dem an der Lipidmembran/Wasser-Grenzschicht lokalisiertem Farbstoff von großem Interesse. Dies war aber aufgrund der Wasserunlöslichkeit des Laurdans nicht realisierbar. Auch das verwandte Prodan, welches sich von Laurdan lediglich durch eine verkürzte Alkylkette unterscheidet (siehe Kapitel 3.5.1), erwies sich als unzureichend wasserlöslich, um die gewünschten Spektren zu erhalten.

$\boldsymbol{n}$-Hexan Als typischer Vertreter eines unpolaren Lösungsmittels wurde $n$-Hexan gewählt (Dielektrizitätskonstante $\varepsilon_{\mathrm{r}}=1.89$ ). Nach elektronischer Anregung des Laurdans in $n$-Hexan findet daher keine dipolare Solvatation statt und es kommt in erster Näherung, d.h. unter Vernachlässigung eventuell stattfindender nicht-dipolarer Solvatationsmechanismen $[14,15,16]$, zu keinem aus Solvatationsdynamik resultierendem Shift des Emissions- bezüglich des Absorptionsmaximums. Das bedeutet, daß die in Abbildung 2.2 dargestellten System-Bad-Wechselwirkungspotentiale des Grund- und angeregten Zustandes nicht gegeneinander verschoben sind. Die dennoch beobachte Verschiebung von $3965 \mathrm{~cm}^{-1}$ resultiert dann vorrangig aus der Relaxation der Überschußenergie aus den Franck-Condon aktiven Moden im angeregten Zustand des Chromophors und den Besetzungsverhältnissen der vibronischen Zustände. Diese Tatsache wird 

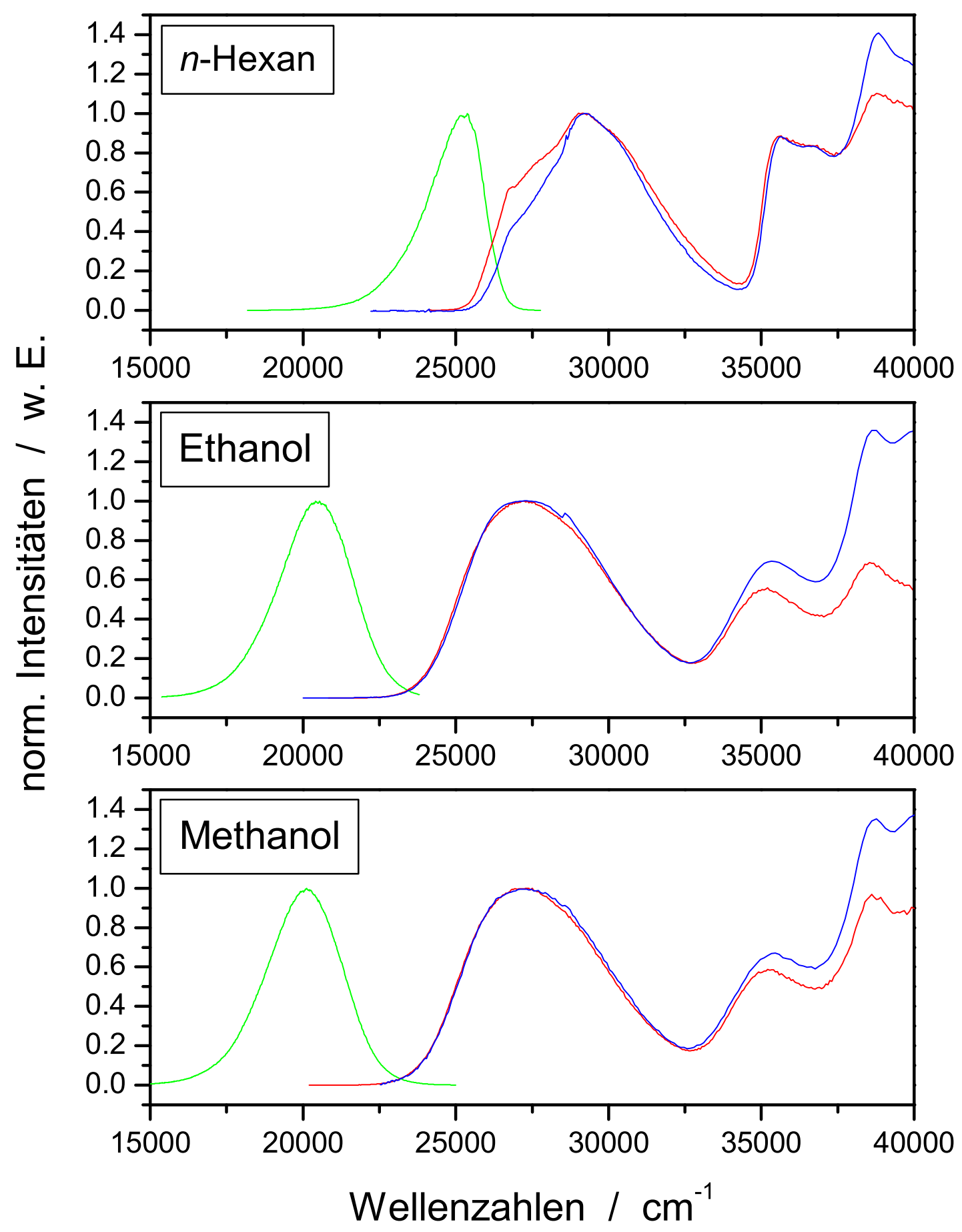

Abbildung 5.1: Absorptions- (blau), Anregungs- (rot) und Emissionsspektren (grün) für Laurdan in n-Hexan, Ethanol und Methanol bei $T=25^{\circ} \mathrm{C}$. 
später ausgenutzt, um die Solvatationskorrelationsfunktion für Laurdan in der Lipidmembran/Wasser-Grenzschicht zu bestimmen (siehe Kapitel 5.4.2).

Die Struktur der Absorptionsbande konnte von Ilich und Prendergast für Prodan, welches einen identischen Chromophor besitzt, mit drei verschiedenen Übergängen bei elektronischer Anregung erklärt werden [222]. Entsprechend läßt sich die Absorptionsbande des Laurdans mit drei Gaußfunktionen wiedergeben. Zwei der elektronischen Übergänge (hier um 326 und $346 \mathrm{~nm}$ zentriert) sind danach einer $\left(\pi^{*} \leftarrow \pi\right)$ - Absorption des Naphthalin-Ringes zuzuordnen, während die Absorption um $372 \mathrm{~nm}$ aus einem $\left(\pi^{*} \leftarrow n\right)$ - Übergang resultiert. Das zusätzlich registrierte Anregungsspektrum entspricht prinzipiell dem Absorptionsspektrum, eine gewisse Abweichung der Bandenformen ist dennoch aus unbestimmten Gründen zu beobachten.

Darüberhinaus wurden temperaturabhängige Messungen der Absorptions- und Emissionsspektren von Laurdan in $n$-Hexan durchgeführt (nicht abgebildet). Es zeigte sich, daß die Absorption im Temperaturbereich von $T=5$ bis $60{ }^{\circ} \mathrm{C}$ nur um ca. $12 \%$ abnimmt, während die Abnahme der Fluoreszenzintensität im gleichen Temperaturintervall mit ca. $45 \%$ deutlich ausgeprägter ausfällt. Die Verringerung der Fluoreszenzintensität mit steigender Temperatur kann möglicherweise auf thermisch aktivierte, strahlungslose Desaktivierungsprozesse zurückgeführt werden. Desweiteren zeigt sich eine minimale Änderung der Bandenlage und Bandenform, was aus der veränderten Population der vibronischen Niveaus mit variierender Temperatur resultiert. Zusammenfassend betrachtet besteht allerdings keine signifikante Temperaturabhängigkeit der spektroskopischen Eigenschaften des Laurdans in $n$-Hexan.

Ethanol Sämtliche Spektren von Laurdan in Ethanol sind im Vergleich zu $n$-Hexan deutlich zu kleineren Wellenzahlen verschoben, was zum einen auf die stark erhöhte Polarität des Ethanols $\left(\varepsilon_{\mathrm{r}}=24.3\right)$ und zum anderen auf die mögliche Ausbildung von Wasserstoffbrücken zurückzuführen ist. Darüberhinaus tritt ein stark erhöhter Stokes-Shift von $6904 \mathrm{~cm}^{-1}$ auf, der hier zusätzlich zur Relaxation von Überschußenergie und den jeweiligen Besetzungsverhältnissen auf dipolare Solvatation schließen läßt. Die in $n$-Hexan noch zu beobachtende Feinstruktur auf Absorptions- und Anregungsspektrum ist in Ethanol aufgrund der erhöhten Solvenskopplung verschwunden (siehe dazu auch Kapitel 2.3). Das Anregungsspektrum ist im Rahmen der Meßgenauigkeit mit dem Absorptionsspektrum identisch.

Methanol Der Wechsel zu Methanol zeigt nur geringe Veränderungen gegenüber Ethanol. Aufgrund der erhöhten Polarität des Methanols $\left(\varepsilon_{\mathrm{r}}=32.6\right)$ und der Ausbildung von Wasserstoffbrückenbindungen sind die Spektren von Laurdan zu niedrigeren Wellenzahlen verschoben und es tritt ein erhöhter Stokes-Shift von $7080 \mathrm{~cm}^{-1}$ auf. 
Tabelle 5.2 faßt die ermittelten Absorptions- und Fluoreszenzmaxima von Laurdan in $\mathrm{cm}^{-1}$ und deren Differenz in den untersuchten Medien zusammen. Zusätzlich sind die jeweiligen Dielektrizitätskonstanten mit aufgeführt [223].

\begin{tabular}{|c|c|c|c|c|}
\hline $\begin{array}{c}\text { Lösungs- } \\
\text { mittel }\end{array}$ & $\boldsymbol{\varepsilon}_{\mathbf{r}}$ & $\tilde{\boldsymbol{\nu}}_{\text {Abs }}^{\max }$ & $\tilde{\boldsymbol{\nu}}_{\mathbf{E m i}}^{\max }$ & $\tilde{\boldsymbol{\nu}}_{\text {Abs }}^{\max }-\tilde{\boldsymbol{\nu}}_{\mathbf{E m i}}^{\max }$ \\
\hline $\begin{array}{c}n \text {-Hexan } \\
\text { Ethanol }\end{array}$ & 1.89 & $29246.8 \mathrm{~cm}^{-1}$ & $25282.0 \mathrm{~cm}^{-1}$ & $3964.8 \mathrm{~cm}^{-1}$ \\
Methanol & 32.6 & $27167.3 \mathrm{~cm}^{-1}$ & $20087.3 \mathrm{~cm}^{-1}$ & $7080.0 \mathrm{~cm}^{-1}$ \\
\hline
\end{tabular}

Tabelle 5.2: Zusammenfassung der Lage der Absorptions- und Emissionsmaxima und deren Differenz von Laurdan in n-Hexan, Methanol und Ethanol.

Die stationären spektroskopischen Eigenschaften des Farbstoffes Laurdan in isotropen Lösungsmitteln machen wie in Kapitel 3.5.1 angesprochen deutlich, daß es sich bei diesem Chromophor um eine äußerst empfindliche Sonde bezüglich der Polarität seiner unmittelbaren Umgebung handelt. Es darf daher angenommen werden, daß die spektroskopischen Eigenschaften des Farbstoffes Laurdan auch innerhalb einer Membran die Polaritätsverhältnisse in der unmittelbaren Umgebung des Chromophors wiederspiegeln und er sich daher zur Bestimmung der genauen Einbautiefe eignet [139,224]. Der beobachtete Stokes-Shift hängt aber nicht nur von der Polarität ab, sondern wird darüberhinaus in erheblichem Maße von der Viskosität und Beweglichkeit der Chromophorumgebung beeinflußt.

\subsection{Untersuchungen an Lipid-Vesikeln}

Durch Einbettung des Laurdans in die Lipidmembran können die Eigenschaften des Farbstoffes an der Lipidmembran/Wasser-Grenzschicht untersucht werden. Um eine erhebliche Beeinflussung der Struktur und des Phasenverhaltens der Modellmembran durch die Einbettung des Fluoreszenzmarkers auszuschließen, wurden zunächst kalorimetrische Messungen durchgeführt (Kapitel 5.2.1). Da sich die stationären Absorptions- und Fluoreszenzeigenschaften des in der Membran verankerten Farbstoffes erheblich von denen in einer homogenen Lösungsmittelumgebung unterscheiden, wurde das System eingehend auf seine stationären spektroskopischen Eigenschaften untersucht. Als erschwerend erwies sich dabei die erhebliche Streuung ultravioletter Strahlung an den untersuchten Vesikeln. Um diese aus der Lipidmembran resultierenden Beiträge einordnen zu können, wurden zunächst Messungen an der reinen, d.h. undotierten, Membran vorgenommen (Kapitel 5.2.2), bevor in Kapitel 5.2.3 auf die spektroskopischen Eigenschaften des Farbstoffes in der Membranumgebung eingegangen wird. 


\subsubsection{Kalorimetrische Ergebnisse}

Da bei der Untersuchung der Solvatationsdynamik an Lipidmembran/WasserGrenzschichten insbesondere die Abhängigkeit vom jeweiligen Phasenzustand der Membran von Interesse ist, soll zunächst der Polymorphismus der untersuchten Modellsysteme betrachtet werden (siehe Kapitel 3.4.3). Dazu wurden temperaturabhängige Wärmekapazitätsprofile mit Hilfe der Dynamischen Differentialkalorimetrie aufgenommen (siehe Kapitel 4.1.3). Hierbei wurde die reine Membran im Vergleich zu unterschiedlich stark mit dem Farbstoff Laurdan markierten Membranen untersucht, um zu bestimmen, in wie weit die Einbettung des Farbstoffes in die Membran deren thermodynamischen Eigenschaften beeinflußt. Die Lösungen wurden mit einer Konzentration von $c_{\mathrm{DMPC}}=10 \mathrm{mM}$ hergestellt. Dabei repräsentiert eine Dotierung von $n_{\text {DMPC }} / n_{\text {Laurdan }}=1000: 1$ die Verhältnisse im TCSPCExperiment, während eine Dotierung von $n_{\text {DMPC }} / n_{\text {Laurdan }}=100: 1$ charakteristisch für die dynamischen Untersuchungen im FUC-Experiment ist.

Die in Abbildung 5.2 dargestellten Wärmekapazitätsprofile zeigen einen für unilamellare DMPC-Vesikel typischen Verlauf. Der mit einer Veränderung der Kopfgruppenregion verbundene Vorphasenübergang von der gelförmigen Phase zur Rippled-Phase $\left(\mathrm{L}_{\beta}, \rightarrow \mathrm{P}_{\beta}\right.$ ) ist nur schwach ausgeprägt und zwischen $11^{\circ} \mathrm{C}$ und $14^{\circ} \mathrm{C}$ angesiedelt. Die Phasenübergangstemperatur wird dabei mit dem ermittel-

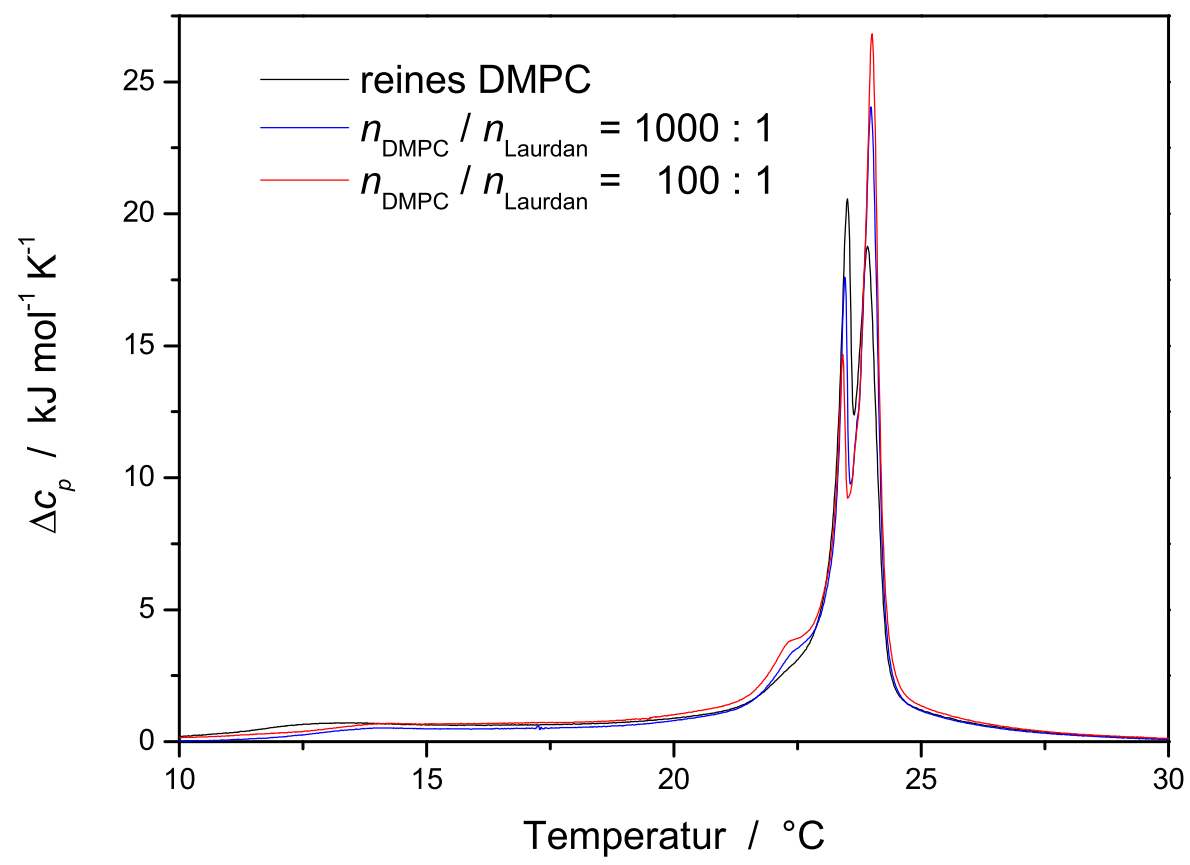

Abbildung 5.2: Wärmekapazitätsprofile für DMPC-Vesikel im Bereich von $T=10$ bis $30^{\circ} \mathrm{C}$ mit unterschiedlicher Farbstoff-Dotierung. 
ten Wendepunkt angegeben. Der Hauptphasenübergang von der Rippled-Phase zur flüssig-kristallinen Phase $\left(\mathrm{P}_{\beta}, \rightarrow \mathrm{L}_{\alpha}\right)$ geht mit einer erheblichen Strukturumwandlung in der Kettenregion der Membran einher und zeigt im Wärmekapazitätsprofil eine entsprechend stark ausgeprägte Wärmekapazitätsänderung. Der Hauptphasenübergang spaltet in zwei Maxima auf, was mit einer schrittweisen Strukturumwandlung interpretiert wird [225]. Als Temperaturangabe für den Hauptphasenübergang dient die Lage des zweiten Maximums.

Die Wärmekapazitätsprofile für reine und unterschiedlich stark markierte DMPCLipidmembran-Vesikel sind grundsätzlich sehr ähnlich. Der Einbau des Farbstoffes Laurdan in die Membran ändert die thermodynamischen Eigenschaften des Systems offensichtlich nur geringfügig. Der Vorphasenübergang wird durch den Einbau des Laurdans geringfügig zu höheren Temperaturen verschoben, während die Lage des Hauptphasenübergangs weitestgehend stabil bleibt. Im Detail ergeben sich die in Tabelle 5.3 ermittelten Phasenübergangstemperaturen. Desweiteren nimmt die Intensität des zweiten Maximus der Wärmekapazitätsprofile im Bereich des Hauptphasenübergangs durch vermehrten Einbau von Laurdan sukzessive zu, während das erste Maximum abnimmt. Bei ca. $22.5^{\circ} \mathrm{C}$ bildet sich eine Schulter aus.

\begin{tabular}{|c|c|c|}
\hline System & $\begin{array}{c}\text { Vorphasenüber- } \\
\text { gangstemperatur }\end{array}$ & $\begin{array}{c}\text { Hauptphasenüber- } \\
\text { gangstemperatur }\end{array}$ \\
\hline reines DMPC & $11.8^{\circ} \mathrm{C}$ & $23.9^{\circ} \mathrm{C}$ \\
$n_{\text {DMPC }} / n_{\text {Laurdan }}=1000: 1$ & $12.8^{\circ} \mathrm{C}$ & $24.0^{\circ} \mathrm{C}$ \\
$n_{\text {DMPC }} / n_{\text {Laurdan }}=100: 1$ & $13.3^{\circ} \mathrm{C}$ & $24.0^{\circ} \mathrm{C}$ \\
\hline
\end{tabular}

Tabelle 5.3: Phasenübergangstemperaturen der untersuchten LipidmembranVesikel bei unterschiedlicher Farbstoff-Dotierung.

Zusammenfassend läßt sich sagen, daß die thermodynamischen Eigenschaften, insbesondere die Lage der Phasenübergangstemperaturen, der untersuchten LipidVesikel durch Einbau der Farbstoffsonde Laurdan nicht erheblich beeinflußt werden und die an der dotierten Membran erhaltenen Ergebnisse daher das Verhalten einer ungestörten Lipidmembran wiederspiegeln sollten. 


\subsubsection{Spektroskopische Eigenschaften der reinen Memb- ran}

In diesem Unterkapitel werden die spektroskopischen Eigenheiten der unmarkierten DMPC-Vesikel präsentiert. Abbildung 5.3 zeigt temperaturabhängige UVVis-Spektren einer wäßrigen, unilamellaren Vesikel-Lösung der Konzentration $c_{\text {DMPC }}=10 \mathrm{mM}$. Im Temperaturintervall von $T=3$ bis $30^{\circ} \mathrm{C}$ wurden Spektren im Abstand von $1{ }^{\circ} \mathrm{C}$, bis $T=60^{\circ} \mathrm{C}$ im Abstand von $5{ }^{\circ} \mathrm{C}$ aufgenommen. Die Spektren zeigen eine stark ansteigende optische Dichte zu kürzeren Wellenlängen, während sie mit steigender Temperatur abnimmt. Dabei spiegelt sich in der Temperaturabhängigkeit der optischen Dichte der charakteristische Polymorphismus der Lipidmembranen wieder. Der rechte Teil der Abbildung verdeutlicht dies durch einen repräsentativen Schnitt durch die UV-Vis-Spektren bei $250 \mathrm{~nm}$.
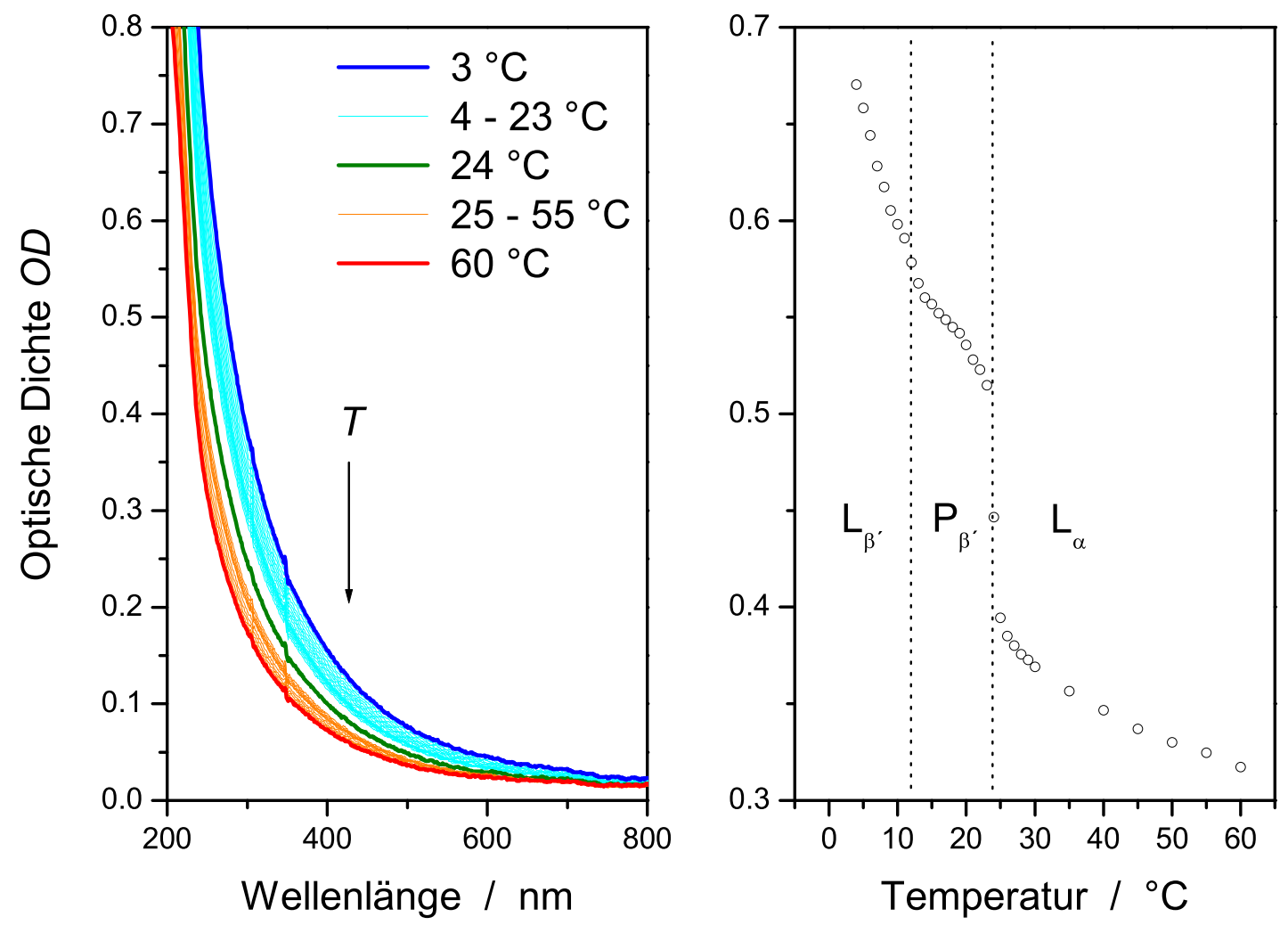

Abbildung 5.3: Links: Temperaturabhängige UV-Vis-Spektren unilamellarer $D M P C$-Vesikel von $T=3{ }^{\circ} \mathrm{C}$ (blau) bis $\mathrm{T}=60^{\circ} \mathrm{C}$ (rot); Rechts: Abhängigkeit der optischen Dichte von der Temperatur bei $\lambda=250 \mathrm{~nm}$. 
Der Hauptphasenübergang $\left(\mathrm{P}_{\beta}, \rightarrow \mathrm{L}_{\alpha}\right)$ bei $23.9^{\circ} \mathrm{C}$ ist deutlich zu erkennen, während der Vorphasenübergang $\left(\mathrm{L}_{\beta}, \rightarrow \mathrm{P}_{\beta}\right.$ ) bei $11.8^{\circ} \mathrm{C}$ lediglich angedeutet erscheint. Die auftretenden Phasen sind entsprechend gekennzeichnet.

Die Ursache der Frequenzabhängigkeit der optischen Dichte wurde anhand konzentrationsabhängiger Messungen untersucht (nicht abgebildet). Die Spektren für $c_{\text {DMPC }}=5,10,15,20,25$ und $30 \mathrm{mM}$ weisen eine Abhängigkeit der gemessenen optischen Dichte von der Konzentration auf, welche auf Mie-Streuung an den Membranvesikeln schließen läßt [226] und nicht auf einer Absorption beruht [27]. Dieser Umstand bedeutet für die vorliegende Arbeit, daß sämtliche Absorptionsspektren des Farbstoffes Laurdan innerhalb einer Membranumgebung bezüglich ihres Streuuntergrundes korrigiert werden müssen.

\subsubsection{Spektroskopische Eigenschaften der markierten Membran}

Zur Charakterisierung der spektroskopischen Eigenschaften des Farbstoffes Laurdan in einer Membranumgebung wurden stationäre Spektren aufgenommen, wobei wiederum die Temperaturabhängigkeit von besonderem Interesse war.

\section{Absorptionsspektren}

In Abbildung 5.4 sind die Absorptionsspektren des Laurdans in DMPC-Vesikeln dargestellt. Bei der untersuchten Lösung handelt es sich um eine Probe der Konzentration $c_{\text {DMPC }}=20 \mathrm{mM}$ mit einem Verhältnis von Lipid zu Farbstoff von $n_{\text {DMPC }} / n_{\text {Laurdan }}=100: 1$. Es wurden Spektren von $T=5$ bis $60^{\circ} \mathrm{C}$ im Abstand von $5{ }^{\circ} \mathrm{C}$ aufgenommen. Die Daten wurden bezüglich des aus der Membran resultierendem Streuuntergrundes korrigiert, so daß die gezeigten Spektren lediglich aus den Absorptionseigenschaften des in der Membran verankerten Laurdans resultieren. Der Peak bei $349 \mathrm{~nm}$ resultiert aus einem Lampenwechsel des verwendeten UV-Vis-Spektrometers.

Es zeigt sich, daß sich die Absorptionseigenschaften des Laurdans oberhalb und unterhalb des Hauptphasenübergangs erheblich voneinander unterscheiden. Beim Überschreiten des Vorphasenübergangs tritt hingegen keine sichtbare Änderung des Absorptionsspektrums auf. Unterhalb des Hauptphasenübergangs, d. h. in der gelförmigen und der Rippled-Phase, zeigt die Absorption ein bimodales Verhalten. Die beiden Maxima sind um $385 \mathrm{~nm}$ und $356 \mathrm{~nm}$ lokalisiert. Mit steigender Temperatur tritt eine minimale Abnahme der Absorption um $385 \mathrm{~nm}$ auf, während die Absorption um $356 \mathrm{~nm}$ geringfügig zunimmt. Dieser Effekt resultiert in einer leichten Verschiebung zu kürzeren Wellenlängen. Beim Übergang in die flüssigkristalline Phase verschwindet das bimodale Verhalten fast vollständig und die 


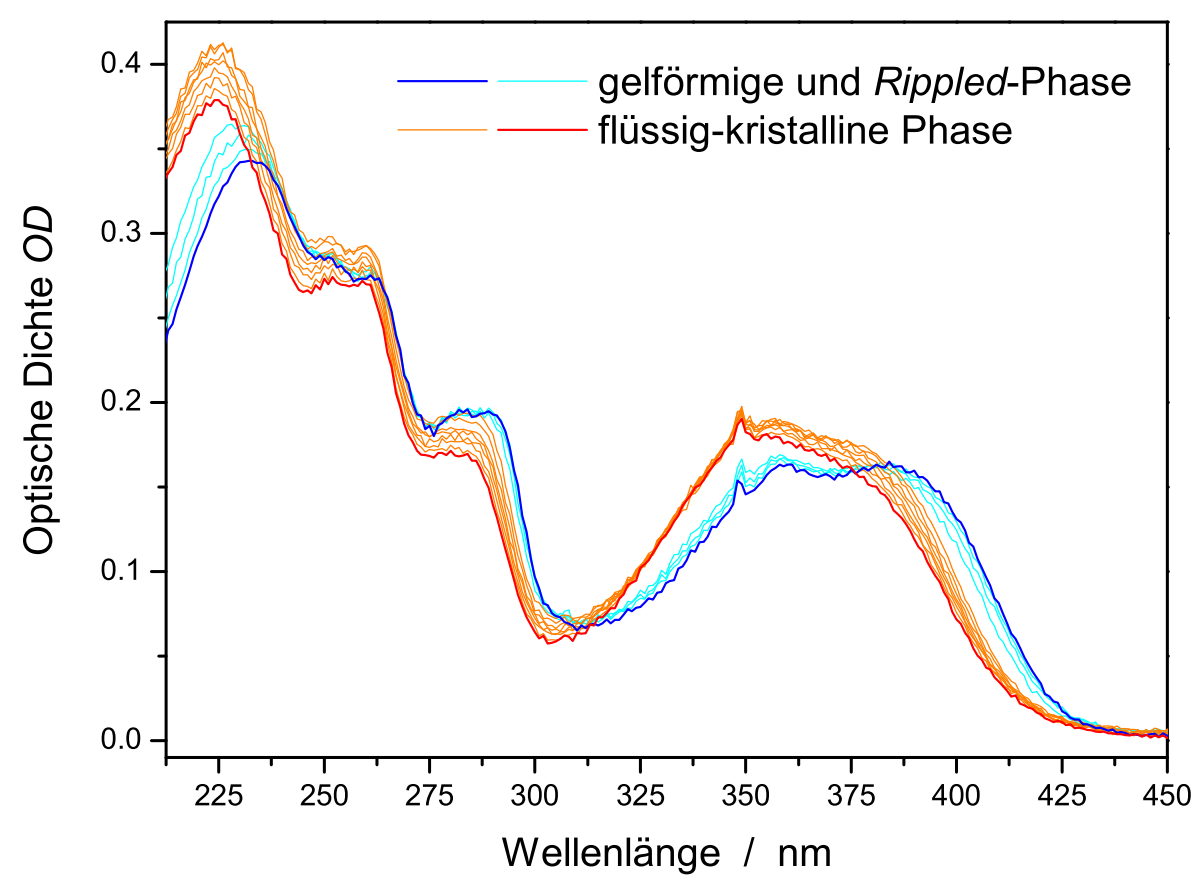

Abbildung 5.4: Temperaturabhängige Absorptionsspektren von Laurdan in $D M P C$ von $T=5^{\circ} \mathrm{C}$ (blau) bis $T=60^{\circ} \mathrm{C}$ (rot).

Spektren werden um ca. $4 \mathrm{~nm}$ zu kürzeren Wellenlängen verschoben, während die optische Dichte etwas zunimmt. Mit fortschreitendem Temperaturanstieg nimmt die optische Dichte auf der roten Flanke der Absorption deutlich ab. In der Abbildung sind zur Verdeutlichung die Spektren bei den Temperaturen $T=5{ }^{\circ} \mathrm{C}$ als Repräsentant für die gelförmige und $T=60^{\circ} \mathrm{C}$ für die flüssig-kristalline Phase farblich hervorgehoben.

Die Ursache der beobachteten Bimodalität wird in der Literatur mit lokal angeregten (LE, Locally Excited) und sogenannten Charge-Transfer-Zuständen (CT) zu erklären versucht $[142,171]$. Bisher konnten aber noch keine eindeutigen Beweise für zwei energetisch benachbarte Zustände in Membranumgebungen erbracht werden. Die photophysikalischen Eigenschaften des Laurdans bedürfen weiterhin genaueren Untersuchungen.

\section{Emissionsspektren}

Temperaturabhängige Fluoreszenzspektren der markierten Membran wurden analog den Absorptionsspektren von $T=5$ bis $60^{\circ} \mathrm{C}$ in Schritten von $5{ }^{\circ} \mathrm{C}$ aufgenommen $\left(\mathrm{RA}, \lambda_{\mathrm{Exc}}=387.5 \mathrm{~nm}, c_{\mathrm{DMPC}}=10 \mathrm{mM}, n_{\mathrm{DMPC}} / n_{\text {Laurdan }}=1000: 1\right)$ und sind in Abbildung 5.5 dargestellt. 


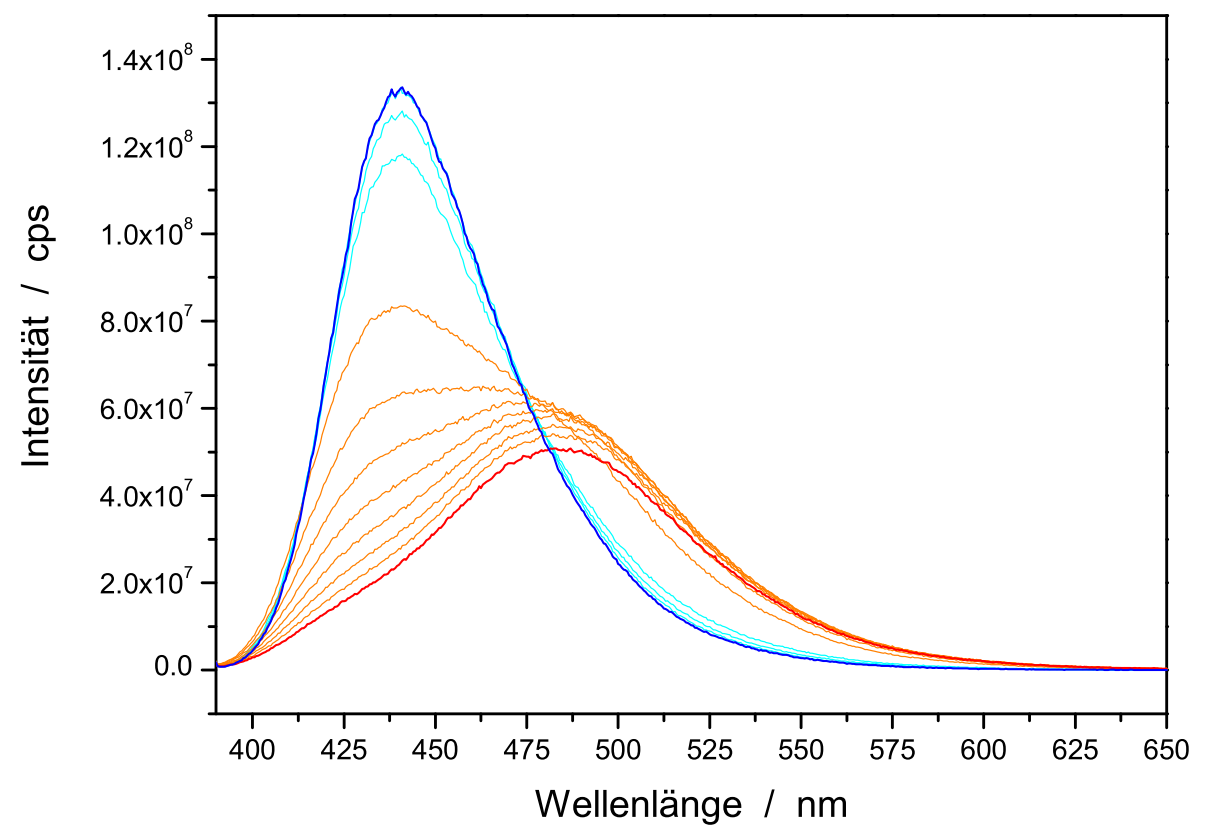

Abbildung 5.5: Temperaturabhängige Fluoreszenzspektren von Laurdan in $D M P C$ von $T=5^{\circ} \mathrm{C}$ (blau) bis $T=60^{\circ} \mathrm{C}$ (rot).

Innerhalb der gelförmigen und Rippled-Phase weist das Emissionsspektrum ein Maximum bei $439.5 \mathrm{~nm}$ auf. Eine Temperaturerhöhung führt vorrangig zu einer Abnahme der Fluoreszenzintensität, während sich das Emissionsspektrum nur minimal verbreitert, aber die Lage des Maximums konstant bleibt. Der Übergang zur flüssig-kristallinen Phase drückt sich darin aus, daß um $485 \mathrm{~nm} \mathrm{zu-}$ sätzliche Fluoreszenz erscheint, die sich zunächst nur als Schulter bemerkbar macht. Mit steigender Temperatur nimmt die bei $439.5 \mathrm{~nm}$ erscheinende Fluoreszenz aber zunehmend ab, so daß die Fluoreszenz bei $485 \mathrm{~nm}$ schließlich dominierend wird. Insgesamt nimmt die Fluoreszenzintensität mit steigender Temperatur aufgrund der immer relevanter werdenden, strahlungslosen Desaktivierungskanäle kontinuierlich ab. Im Gegensatz zu den temperaturabhängigen Absorptionsspektren, die beim Überschreiten des Hauptphasenübergangs eine plötzliche Änderung der spektralen Eigenschaften aufweisen, wird der Übergang hier lediglich von einer kontinuierlichen Änderung der Fluoreszenzspektren begleitet (siehe Abbildung 5.5). Es ist zu bemerken, daß die gezeigten temperaturabhängigen Fluoreszenzspektren von Laurdan in DMPC/Wasser-Grenzschichten keinen isosbestischen Punkt aufweisen, wie in der Literatur bereits berichtet wurde [142]. 


\section{Zusammenfassung}

Um die spektroskopischen Eigenschaften des Laurdans in den unterschiedlichen Phasenzuständen der Membran nochmals zu verdeutlichen, sind in Abbildung 5.6 für zwei ausgewählte Temperaturen die Absorptions-, Emissions- und zusätzlich die Anregungsspektren (RA, $\lambda_{\text {Det }}=440 \mathrm{~nm}\left(T=10^{\circ} \mathrm{C}\right) \mathrm{bzw} .480 \mathrm{~nm}\left(T=60{ }^{\circ} \mathrm{C}\right)$, $\left.c_{\text {DMPC }}=10 \mathrm{mM}, n_{\mathrm{DMPC}} / n_{\text {Laurdan }}=1000: 1\right)$ dargestellt. Dabei repräsentieren die Messungen bei $T=10^{\circ} \mathrm{C}$ das typische spektrale Verhalten in der gelförmigen und Rippled-Phase, während $T=60^{\circ} \mathrm{C}$ typisch für die flüssig-kristalline Phase ist.

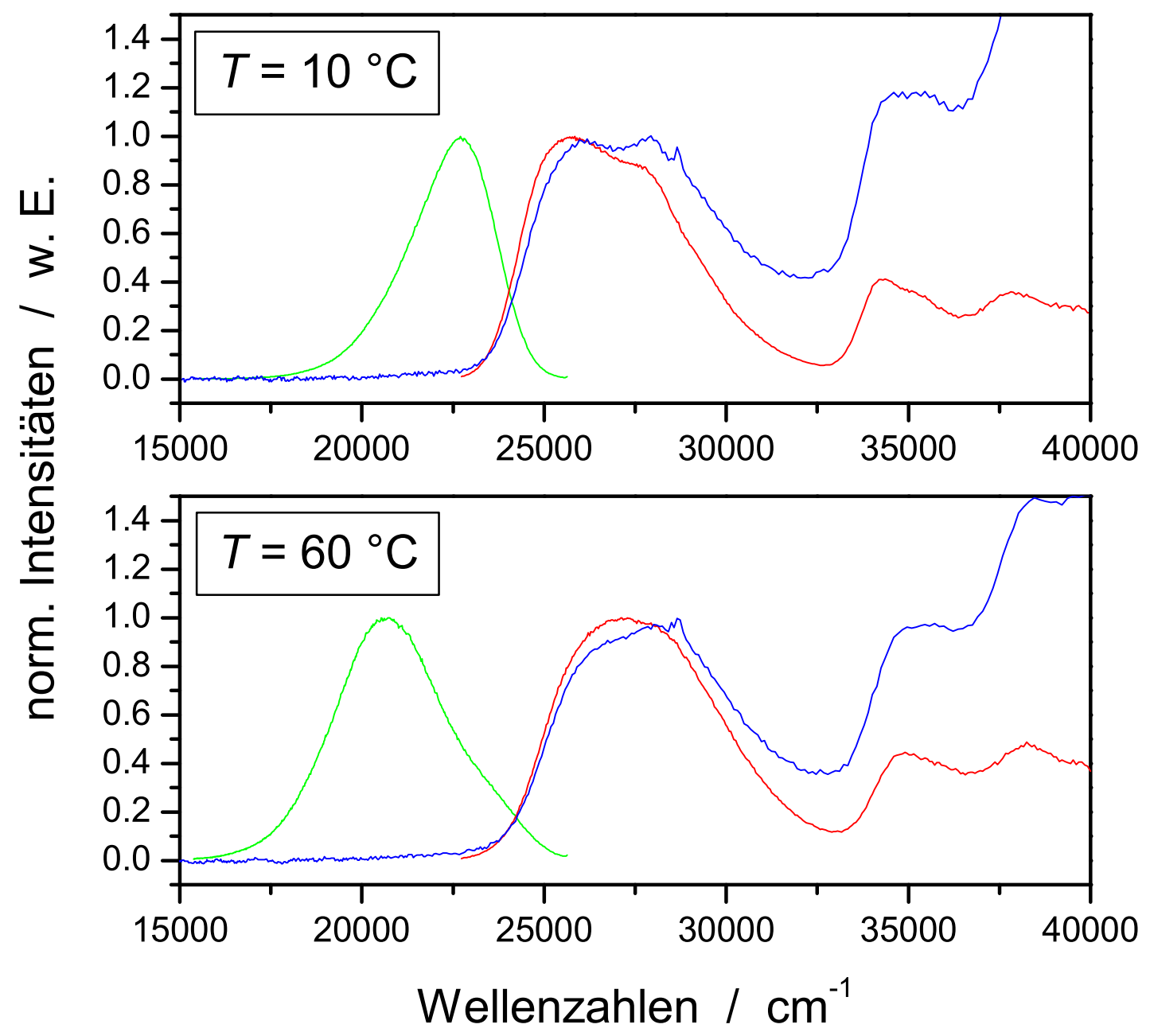

Abbildung 5.6: Absorptions- (blau), Anregungs- (rot) und Emissionsspektren (grün) für Laurdan in $D M P C$ bei $T=10^{\circ} \mathrm{C}$ und $\mathrm{T}=60^{\circ} \mathrm{C}$. 
Die Anregungsspektren weisen zwar erhebliche Abweichungen von ihren zugehörigen Absorptionsspektren auf, das weitestgehende Verschwinden der Bimodalität beim Überschreiten des Hauptphasenübergangs wird aber auch hier wiedergegeben.

Die mit den stationären spektroskopischen Methoden gemachten Beobachtungen weisen darauf hin, daß der Chromophor vergleichsweise tief innerhalb der Kopfgruppenregion der Lipidmembran verankert ist, was bereits in einer Vielzahl von Veröffentlichungen gezeigt wurde [139,142,165]. Diese Arbeiten propagieren eine ungefähre Lage des Chromophors innerhalb der Membran, wie sie in Abbildung 3.6 skizziert ist. Durch diese tiefe Einbaulage erklärt sich vermutlich auch die fehlende Empfindlichkeit der spektroskopischen Eigenschaften des Chromophors bezüglich des Vorphasenübergangs. Die erheblichen Veränderungen der spektroskopischen Eigenschaften beim Überschreiten des Hauptphasenübergangs wurden unter verschiedenen Aspekten in der Literatur diskutiert. Neben der bereits angesprochenen Beteiligung von Charge-Transfer-Zuständen [171], wurde unter anderem untersucht, ob die beobachten Effekte auf veränderte Solvatationsprozesse oder auf eine Relokalisierung des Chromophors innerhalb der Membran zurückzuführen sind [138]. Darüberhinaus läßt sich vermuten, daß die beobachtete Verschiebung der Absorptionsspektren zu kürzeren Wellenlängen beim Überschreiten des Hauptphasenübergangs eine direkte Konsequenz der erheblichen Vergrößerung der pro Lipidmolekül beanspruchten Fläche in der flüssig-kristallinen Phase ist [79]. Die geringere Packungsdichte der Lipide führt zu einer verminderten Polarität in der unmittelbaren Umgebung des Chromophors, was zu der beobachteten spektralen Verschiebung führt. Im Gegenzug führen die beim Hauptphasenübergang beobachteten Packungseffekte jedoch auch zu einem tieferen Eindringen von Wassermolekülen in die Kopfgruppenregion der Lipide [165], weswegen die Beteiligung von Wasser bei der Solvatation des in der Membran verankerten Chromophors vorrangig in der flüssig-kristallinen Phase als relevant erachtet wird. Weiterhin erfährt der Chromophor durch das Schmelzen der Lipidketten eine stark erhöhte Mobilität, was insbesondere in Anisotropiemessungen anschaulich gezeigt werden kann (siehe dazu Kapitel 5.3). Diese Mobilitätssteigerung des Chromophors im Zusammenspiel mit der erhöhten Wasserdichte führt vermutlich zu dem deutlich vergrößerten Stokes-Shift in der flüssig-kristallinen Phase. Eine genauere Untersuchung und Bestätigung der gefundenen Effekte wird im folgenden Kapitel mit zeitaufgelösten Untersuchungen zu den Anisotropieeigenschaften und in Kapitel $5.4 \mathrm{zu}$ den Solvatationseigenschaften des Chromophors in der Membran vorgenommen. 


\subsection{Anisotropieeigenschaften}

Die zeitabhängige Anisotropie eines in einer Lipidmembran eingebetten Farbstoffes ist von besonderem Interesse, da sie Informationen über die zulässigen Dynamiken des Farbstoffes in der Membran in Abhängigkeit ihres Phasenzustandes liefert. Die Grundzüge der zugrundeliegenden Theorie einer eingeschränkten Rotationsmöglichkeit von Chromophoren wurde bereits 1975 von Wahl vorgestellt [227,228] und 1977 von Kinosita et al. auf die eingeschränkten Rotationsmöglichkeiten in Membranen angewendet [229]. Die Theorie ist als Wobble-inCone-Modell bekannt geworden [158,178,229, 230,231,232,233,234]. Wenn $r_{0}$ die anfängliche Anisotropie in einem System zur Zeit $t=0$ bezeichnet und $r_{\infty}$ die zu langen Zeiten limitierende Anisotropie, dann ist der Quotient $r_{\infty} / r_{0}$ in eingeschränkten Umgebungen im Gegensatz zu isotropen und wenig viskosen Medien nicht gleich Null, sondern stellt ein Maß für die durch die Umgebung aufgeprägte Einschränkung der Bewegungsmöglichkeit des Chromophors dar. Der zeitliche Verlauf der Anisotropiefunktion $r(t)$ repräsentiert dann die für den Chromophor charakteristische, diffusive „Wackelbewegung“ in einem Konus.

Mit Hilfe des zeitkorrelierten Einzelphotonenzählexperimentes wurden im Maximum der Fluoreszenz $\lambda_{\max }$ für Temperaturen von $T=5$ bis $60^{\circ} \mathrm{C}$ jeweils mit senkrechter und paralleler Anregung bezüglich der Detektion, die Fluoreszenzabklingkurven $I_{\perp}(t)$ bzw. $I_{\|}(t)$ gemessen (siehe auch Kapitel 4.2.3). Die Konzentration der Meßlösung betrug $c_{\text {DMPC }}=10 \mathrm{mM}$ bei einem Membran-zu-Farbstoffverhältnis von $n_{\text {DMPC }} / n_{\text {Laurdan }}=1000: 1$. Aus den erhaltenen Fluoreszenzabklingkurven läßt sich die zeitabhängige Anisotropiefunktion $r(t)$ berechnen:

$$
r(t)=\frac{I_{\|}(t)-I_{\perp}(t)}{I_{\|}(t)+2 I_{\perp}(t)} .
$$

Die so ermittelten Funktionen $r(t)$ in Abhängigkeit der Temperatur sind in Abbildung 5.7 dargestellt. Es zeigt sich, daß alle Funktionen in Abhängigkeit von der Temperatur bei einer Anfangsanisotropie $r_{0}$ zwischen 0.4 und 0.3 beginnen und mit fortschreitender Zeit nach Anregung biexponentiell abklingen. Dabei strebt die Anisotropie unterhalb des Hauptphasenübergangs gegen einen von Null verschiedenen Wert $r_{\infty}$, während oberhalb des Hauptphasenübergangs eine deutliche stärkere Abnahme bis annähernd auf Null zu beobachten ist. Die für eine biexponentielle Anpassung nach

$$
r(t)=\sum_{i=1}^{2} A_{i} \exp \left(-\frac{t}{\tau_{i}}\right)+r_{\infty}
$$

ermittelten Anpassungsparameter, sowie die daraus bestimmten Anfangsanisotropien $r_{0}$ sind in Tabelle 5.4 zusammengefaßt. 


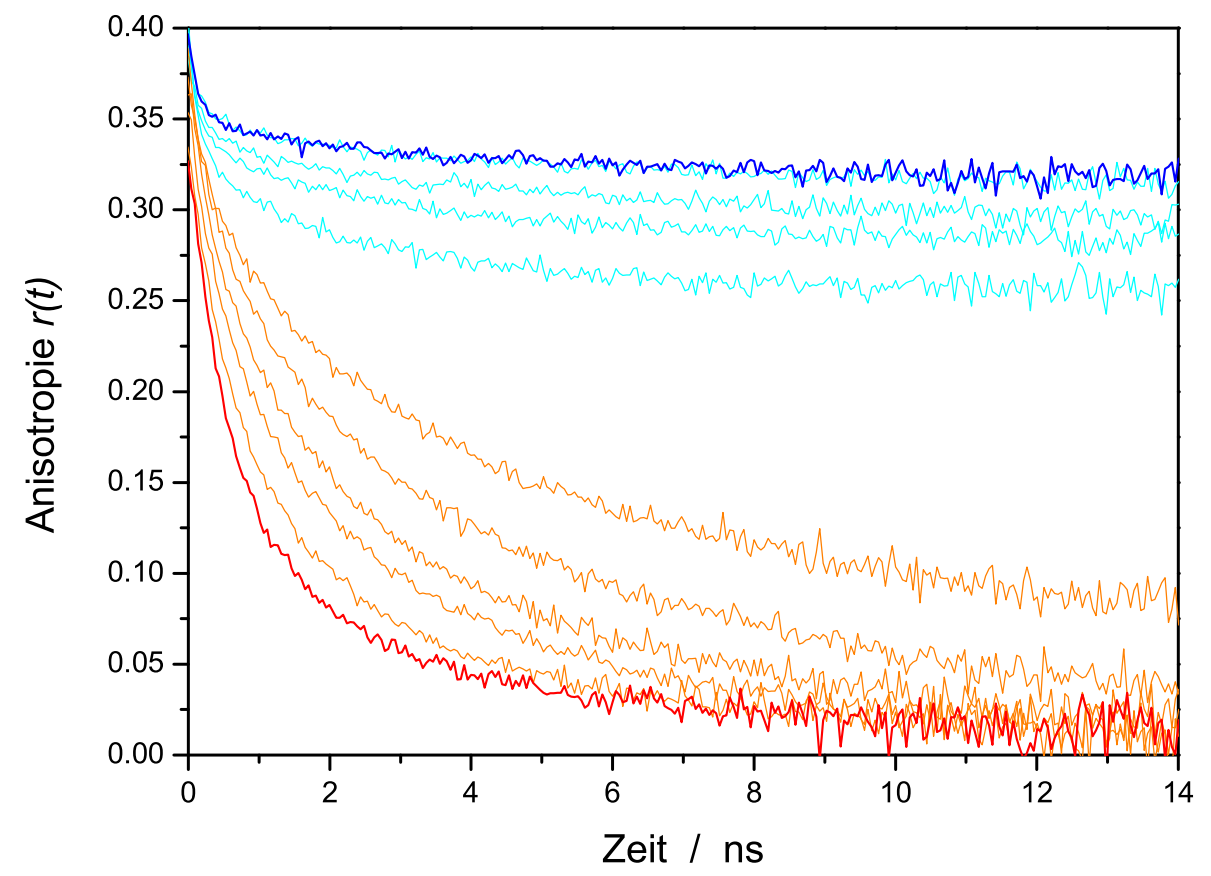

Abbildung 5.7: Für Laurdan in DMPC erhaltene Anisotropiefunktionen $r(t)$ für Temperaturen von $T=5{ }^{\circ} \mathrm{C}$ (blau) bis $T=60^{\circ} \mathrm{C}$ (rot).

Aus $r_{0}$ und $r_{\infty}$ ergibt sich nach dem Wobble-in-Cone-Modell der sogenannte Ordnungsparameter $S$ nach:

$$
S=\sqrt{\frac{r_{\infty}}{r_{0}}} .
$$

Dieser Ordnungsparameter $S$ ist über die Beziehung

$$
\frac{1}{2} \cos \theta_{\mathrm{c}}\left(1+\cos \theta_{\mathrm{c}}\right)=S
$$

mit dem interessierenden Konuswinkel $\theta_{c}$ verknüpft. Der Konuswinkel beschreibt den Winkel zwischen dem Emissionsübergangsmoment des Chromophors und der Membrannormalen und reflektiert die Amplitude der möglichen Rotationsbewegung im Wobble-in-Cone-Modell.

Von Lipari und Szabo wurde gezeigt, daß die Wobbling-Diffusion-Konstante $D_{\mathrm{w}}$ über folgende Beziehung berechnet werden kann, wobei $x_{\mathrm{c}}$ für $\cos \theta_{\mathrm{c}}$ steht [230]:

$$
\begin{aligned}
D_{\mathrm{w}} \cdot F= & -x_{\mathrm{c}}^{2}\left(1+x_{\mathrm{c}}\right)^{2} \frac{\ln \left(\frac{\left(1+x_{\mathrm{c}}\right)}{2}\right)+\frac{\left(1-x_{\mathrm{c}}\right)}{2}}{2\left(1-x_{\mathrm{c}}\right)} \\
& +\left(1-x_{\mathrm{c}}\right) \frac{6+8 x_{\mathrm{c}}-x_{\mathrm{c}}^{2}-12 x_{\mathrm{c}}^{3}-7 x_{\mathrm{c}}^{4}}{24} .
\end{aligned}
$$




\begin{tabular}{|c|c|c|c|c|c|c|c|}
\hline $\boldsymbol{T} /{ }^{\circ} \mathbf{C}$ & $\boldsymbol{\lambda}_{\max } / \mathbf{n m}$ & $\boldsymbol{A}_{\mathbf{1}}$ & $\boldsymbol{\tau}_{\mathbf{1}} / \mathbf{n s}$ & $\boldsymbol{A}_{\mathbf{2}}$ & $\boldsymbol{\tau}_{\mathbf{2}} / \mathbf{n s}$ & $\boldsymbol{r}_{\boldsymbol{\infty}}$ & $\boldsymbol{r}_{\mathbf{0}}$ \\
\hline 5 & 445 & 0.048 & 0.174 & 0.030 & 4.72 & 0.317 & 0.395 \\
10 & 445 & 0.047 & 0.191 & 0.034 & 5.10 & 0.312 & 0.393 \\
15 & 445 & 0.044 & 0.252 & 0.042 & 5.10 & 0.294 & 0.380 \\
20 & 445 & 0.053 & 0.172 & 0.052 & 3.13 & 0.283 & 0.389 \\
22.5 & 445 & 0.075 & 0.154 & 0.069 & 2.52 & 0.257 & 0.402 \\
25 & 475 & 0.087 & 0.402 & 0.217 & 4.79 & 0.073 & 0.377 \\
30 & 485 & 0.113 & 0.703 & 0.228 & 5.06 & 0.024 & 0.365 \\
35 & 490 & 0.144 & 0.725 & 0.207 & 4.41 & 0.012 & 0.362 \\
40 & 490 & 0.139 & 0.588 & 0.201 & 3.58 & 0.012 & 0.352 \\
50 & 490 & 0.185 & 0.705 & 0.136 & 3.60 & 0.011 & 0.332 \\
60 & 490 & 0.216 & 0.627 & 0.102 & 3.88 & 0.010 & 0.327 \\
\hline
\end{tabular}

Tabelle 5.4: Aus biexponentieller Anpassung der Anisotropiefunktion erhaltene Anpassungsparameter in Abhängigkeit der Temperatur.

Darin bezeichnet $F$ das Integral unterhalb der Anisotropiefunktion:

$$
F=\int_{0}^{\infty} \frac{r(t)-r_{\infty}}{r_{0}} \mathrm{~d} t
$$

Der Kehrwert der Wobbling-Diffusion-Konstante $D_{\mathrm{w}}$ ist dabei proportional zur Mikroviskosität $\eta_{\mathrm{w}}$.

Desweiteren wurde das Konzept der Rotationsdiffusion auf Membranen übertragen $[235,236,237]$. Es zeigte sich, daß die zugehörige Rotationsdiffusionskonstante $D_{\mathrm{R}}$, welche die Rotation um die lange Probenachse des Chromophors beschreibt, über die Steigung der Anisotropiefunktion $r(t)$ zum Zeitpunkt $t=0$ berechnet werden kann:

$$
D_{\mathrm{R}}=-\left.\frac{1}{6 r_{0}} \frac{\partial r(t)}{\partial t}\right|_{t=0} .
$$

Für biexponentielles Abklingverhalten von $r(t)$ ergibt sich daraus der analytische Ausdruck:

$$
D_{\mathrm{R}}=\frac{1}{6 r_{0}} \sum_{i=1}^{2} \frac{A_{i}}{\tau_{i}} .
$$

Mit den aus biexponentiellen Anpassungen der Anisotropiefunktionen erhaltenen Anpassungsparameter aus Tabelle 5.4 wurden die Ordnungsparameter $S$, die Konuswinkel $\theta_{\mathrm{c}}$, die Flächen $F$ unterhalb der Anisotropiefunktionen, die Wobblingdiffusionskonstanten $D_{\mathrm{w}}$ und die Rotationsdiffusionskonstanten $D_{\mathrm{R}}$ in Abhängigkeit der Temperatur berechnet. Die erhaltenen Werte sind in Tabelle 5.5 zusammengefaßt. 


\begin{tabular}{|c|c|c|c|c|c|}
\hline $\boldsymbol{T} /{ }^{\circ} \mathbf{C}$ & $\boldsymbol{S}$ & $\boldsymbol{\theta}_{\mathbf{c}} /{ }^{\circ}$ & $\boldsymbol{F} / \mathbf{n s}$ & $\begin{array}{c}\boldsymbol{D}_{\mathbf{w}} \\
/ \mathbf{1 0}^{-\mathbf{3}} \mathbf{n s}^{-\mathbf{1}}\end{array}$ & $\begin{array}{c}\boldsymbol{D}_{\mathbf{R}} \\
/ \mathbf{1 0}^{-\mathbf{2}} \mathbf{n s}^{-\mathbf{1}}\end{array}$ \\
\hline 5 & 0.896 & 21.7 & 0.375 & 21.1 & 12.0 \\
10 & 0.891 & 22.2 & 0.465 & 18.5 & 10.6 \\
15 & 0.879 & 23.5 & 0.593 & 17.8 & 8.1 \\
20 & 0.854 & 25.9 & 0.443 & 34.1 & 13.9 \\
22.5 & 0.800 & 30.7 & 0.464 & 59.2 & 21.4 \\
25 & 0.441 & 55.7 & 2.848 & 55.5 & 11.5 \\
30 & 0.257 & 68.0 & 3.372 & 66.5 & 9.4 \\
35 & 0.179 & 73.8 & 2.807 & 86.7 & 11.3 \\
40 & 0.184 & 73.4 & 2.276 & 106.5 & 13.9 \\
50 & 0.180 & 73.7 & 1.871 & 130.0 & 15.1 \\
60 & 0.171 & 74.3 & 1.620 & 151.1 & 18.9 \\
\hline
\end{tabular}

Tabelle 5.5: Auswertung der Anisotropiefunktion nach dem Wobble-in-ConeModell in Abhängigkeit der Temperatur.

In Abbildung 5.4 sind die ermittelten Konuswinkel $\theta_{c}$ sowie die limitierenden Anisotropien $r_{\infty}$ gegen die Temperatur aufgetragen. Es zeigt sich eine starke Korrelation der zwei Größen, welche beide das charakteristische Verhalten von Lipidmembranen in Abhängigkeit ihrer thermodynamischen Phase beschreiben. Unterhalb des Hauptphasenübergangs macht sich die erhebliche Einschränkung der möglichen Rotationsdynamik des eingebetteten Farbstoffes durch die dicht gepackten Lipidmoleküle im verminderten Konuswinkel bemerkbar. Entsprechend fällt die Anisotropiefunktion auch für lange Zeiten, d. h. im Bereich der Fluoreszenzlebensdauer, nicht auf Null ab. Oberhalb des Hauptphasenübergangs tritt eine stark veränderte Struktur der Membran auf, was sich in der erhöhten Bewegungsmöglichkeit des Chromophors und dementsprechend in einem Konuswinkel von ca. $75^{\circ}$ ausdrückt. Die freie Bewegungsmöglichkeit des Farbstoffes erlaubt hier das Abfallen der Anisotropie auf Null innerhalb des untersuchbaren Zeitfensters. Der Übergang erfolgt in einem relativ schmalen Temperaturbereich, der um $24^{\circ} \mathrm{C}$ lokalisiert ist, was der Temperatur des Hauptphasenübergangs entspricht. $\mathrm{Zu}$ bemerken ist, daß beim Vorphasenübergang, der laut kalorimetrischer Untersuchungen um $13^{\circ} \mathrm{C}$ auftritt (siehe Kapitel 5.2.1), keinerlei Änderung des Anisotropieverhaltens beobachtet werden kann. Die Ursache liegt darin, daß der Vorphasenübergang vorrangig mit einer Umstrukturierung der Kopfgruppenregion einhergeht und damit keinerlei Einfluß auf die Bewegungsmöglichkeiten des in der Membran befindlichen Chromophors hat. 


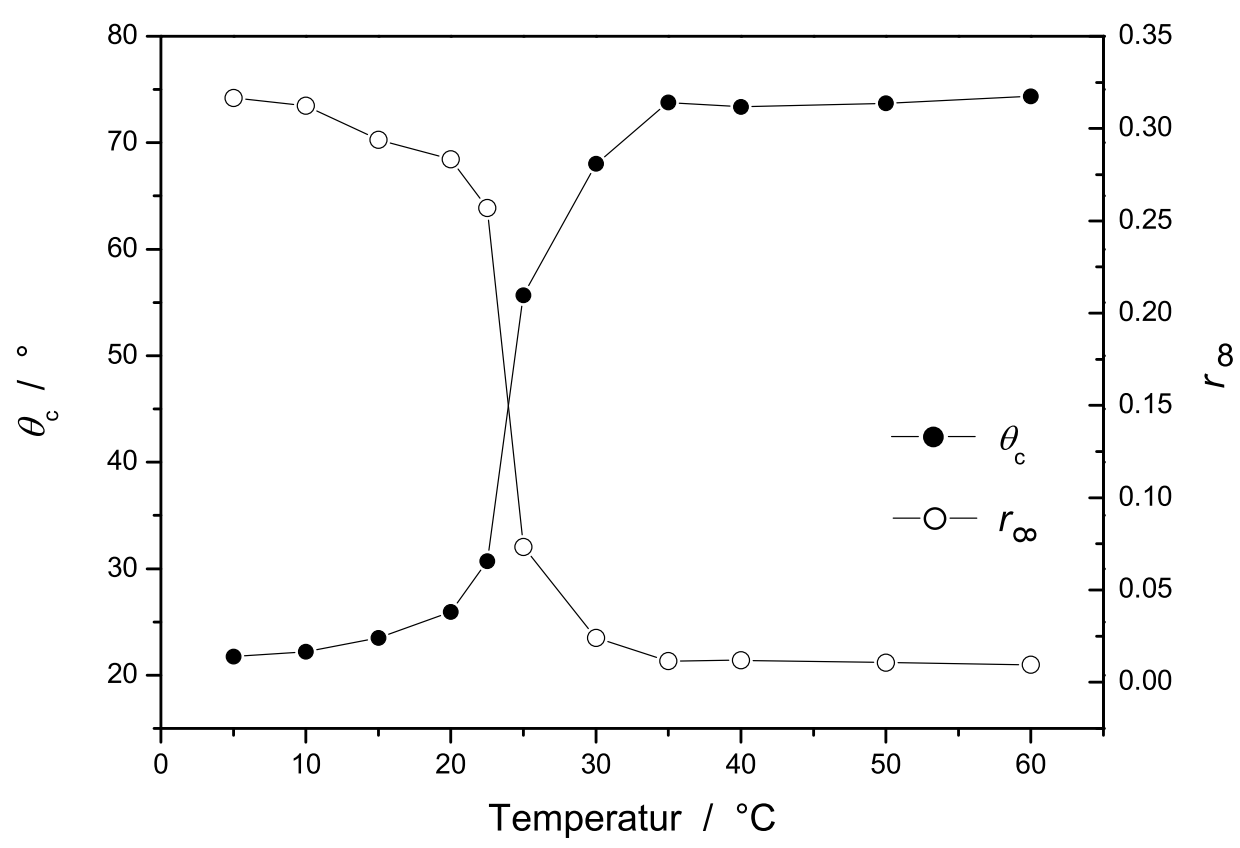

Abbildung 5.8: Konuswinkel $\theta_{c}$ und limitierende Anisotropie $r_{\infty}$ in Abhängigkeit der Temperatur.

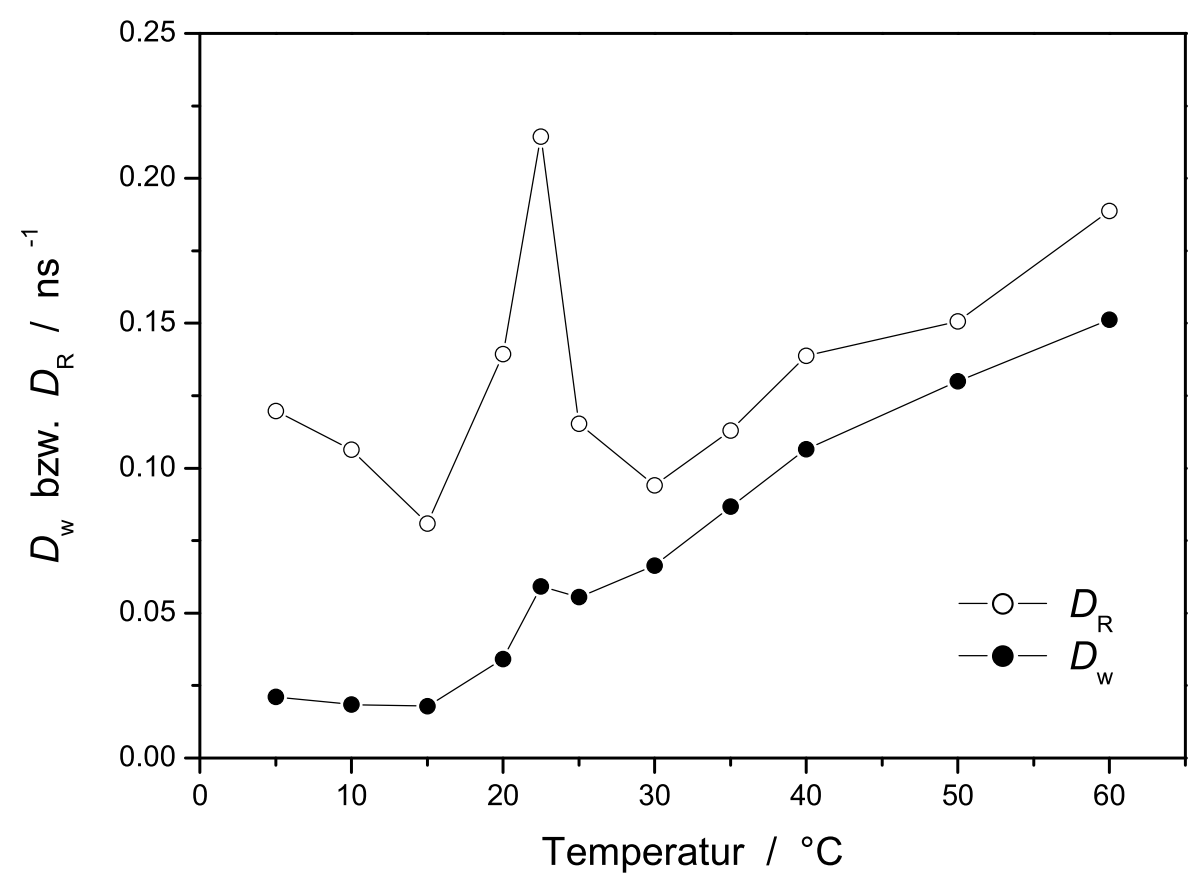

Abbildung 5.9: Darstellung der Wobbling-Diffusions- und der Rotationsdiffusionskonstante in Abhängigkeit der Temperatur. 
Die Temperaturabhängigkeit der ermittelten Diffusionskonstanten $D_{\mathrm{w}}$ und $D_{\mathrm{R}}$ ist in Abbildung 5.9 dargestellt. Die Wobbling-Diffusionskonstante erscheint unterhalb des Phasenübergangs auf weitestgehend konstantem, aber niedrigem Niveau und steigt oberhalb des Phasenübergangs deutlich an. Ein ähnliches Verhalten wurde auch für den Farbstoff DPH (1,6-Diphenyl-1,3,5-hexatrien) in DPPC-Membranen beobachtet [231]. Die Rotationsdiffusionskonstante $D_{\mathrm{R}}$ zeigt ein deutlich unterschiedliches Verhalten. Sie fällt unterhalb des Hauptphasenübergangs geringfügig ab und wächst oberhalb mit gleicher Steigung wie $D_{\mathrm{w}}$ an. Am Phasenübergang selbst zeigt sich aber eine deutliches Maximum der Rotationsdiffusionskonstanten als ausgeprägter Peak. Eine weiterreichende Diskussion der Anisotropieeigenschaften erfolgt im Zusammenhang mit der Diskussion der Solvatationsdynamik in Kapitel 5.5.

\subsection{Solvatationsdynamik}

Die Solvatation an der Lipidmembran/Wasser-Grenzschicht wurde aufgrund ihrer viele Größenordnungen überstreichenden Dynamik mit zwei unterschiedlichen Methoden untersucht. Bis zu mehreren zehn Nanosekunden kam das zeitkorrelierte Photonenzählen mit einer Zeitauflösung von ca. 200 ps zur Anwendung. Die obere zeitliche Grenze stellt dabei die endliche Fluoreszenzlebensdauer des verwendeten Farbstoffes dar. Um auch die ultraschnelle Solvatationsdynamik bis ca. 0.5 ps auflösen zu können, wurden Experimente mit der Fluoreszenzkonversionsmethode durchgeführt.

\subsubsection{Zeitaufgelöste Fluoreszenzspektren}

Mit der Methode des zeitkorrelierten Einzelphotonenzählens wurden frequenzaufgelöste Kinetiken von 400 bis $615 \mathrm{~nm}$ im Abstand von $5 \mathrm{~nm}$ bei einer Anregungswellenlänge von $400 \mathrm{~nm}$ aufgenommen (siehe auch Kapitel 4.2.3). Die Konzentration der Meßlösung betrug $c_{\mathrm{DMPC}}=10 \mathrm{mM}$ bei einem Membran-zuFarbstoffverhältnis von $n_{\text {DMPC }} / n_{\text {Laurdan }}=1000: 1$. Es wurden Kinetiken für Temperaturen von $T=5$ bis $60^{\circ} \mathrm{C}$ im Abstand von $5^{\circ} \mathrm{C}$ aufgenommen. Nach spektraler Korrektur konnten so zeitaufgelöste Fluoreszenzspektren rekonstruiert werden. Repräsentativ sind zeitabhängige Spektren für $T=10^{\circ} \mathrm{C}$ (gelförmige Phase) und $T=60^{\circ} \mathrm{C}$ (flüssig-kristalline Phase) in Abbildung 5.10 als Konturplot dargestellt.

Es ist bereits qualitativ zu erkennen, daß bei $T=10^{\circ} \mathrm{C}$ einen deutlich kleinerer dynamischer Stokes-Shift zu beobachten ist und die Spektren deutlich schmaler sind als bei $T=60^{\circ} \mathrm{C}$. Desweiteren dauert die abgestrahlte Fluoreszenz über einen längeren Zeitraum an. 

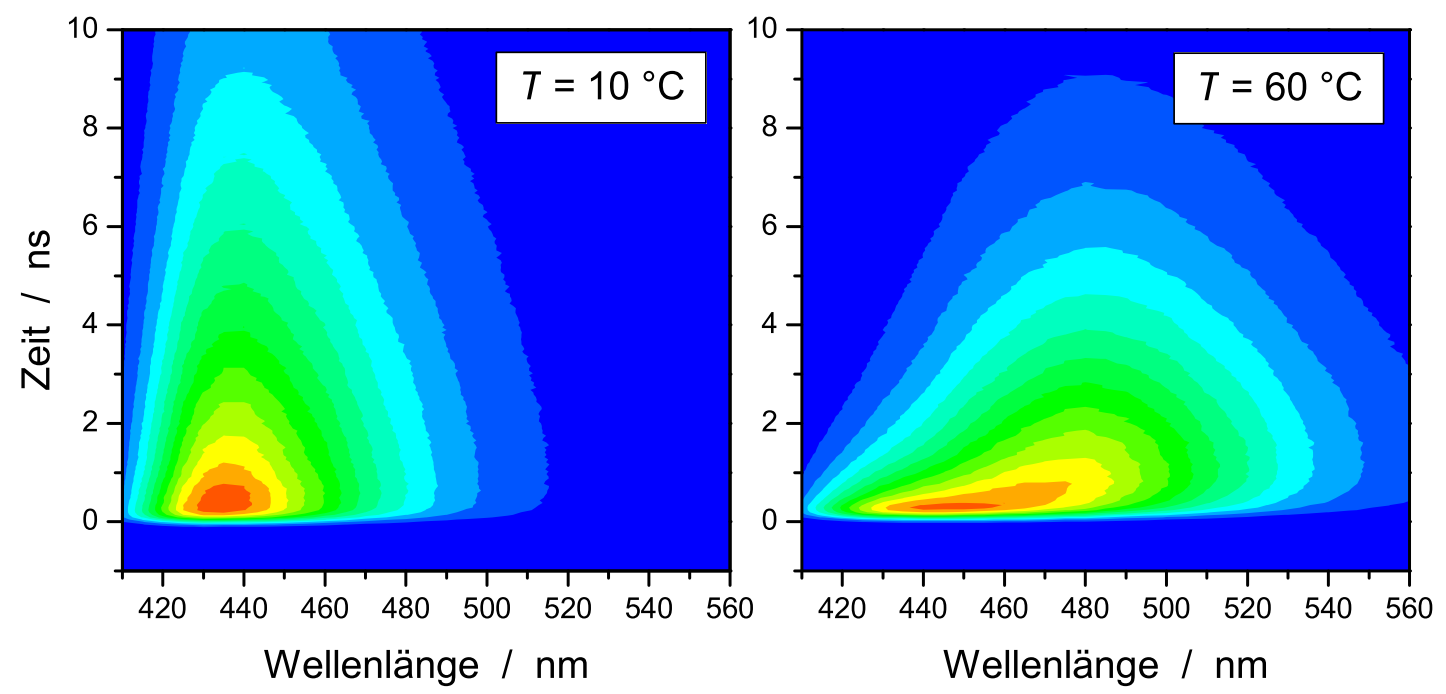

Abbildung 5.10: Zeitaufgelöste Fluoreszenzspektren von Laurdan in DMPC bei $T=10^{\circ} \mathrm{C}$ und $\mathrm{T}=60^{\circ} \mathrm{C}$ in Konturdarstellung.

Im folgenden sollen diese zeitaufgelösten Spektren, sowie die nicht dargestellten Messungen bei $T=5{ }^{\circ} \mathrm{C}$ und $T=15$ bis $55^{\circ} \mathrm{C}$, eingehend analysiert werden. Die Analyse folgt dabei prinzipiell dem Verfahren von Maroncelli und Fleming [238].

\section{Anpassung der zeitabhängigen Spektren}

Um die erhaltenen Spektren untereinander quantitativ vergleichen zu können, wurden die Wellenlängen zunächst in eine zur Energie proportionale Einheit umgewandelt, d. h. Wellenzahlen in $\mathrm{cm}^{-1}$.

Die zeitaufgelösten Spektren unterhalb des Hauptphasenübergangs, d. h. in der gelförmigen und Rippled-Phase, konnten mit einer zeitabhängigen Log-NormalFunktion wiedergegeben werden:

$$
g(\tilde{\nu}, t)=g_{0}(t) \cdot \exp \left(-\ln 2\left[\frac{\ln \left(1+\frac{2 b(t)\left(\tilde{\nu}-\tilde{\nu}_{0}(t)\right)}{\Delta(t)}\right)}{b(t)}\right]^{2}\right)
$$

Dabei bezeichnet $g_{0}$ die Amplitude, $b$ die Asymmetrie, $\Delta$ die Breite und $\tilde{\nu}_{0}$ die Position des Emissionsmaximums zur Zeit $t$. Eine Log-Normal-Funktion stellt eine asymmetrische Linienfunktion dar, die im Grenzfall $b \rightarrow 0$ in eine Gaußfunktion übergeht [187]. 
Oberhalb des Hauptphasenübergangs, d. h. in der flüssig-kristallinen Phase, war die Anpassung mit einer zeitabhängigen Log-Normal-Funktion nicht befriedigend. Hier wurden die Spektren mit einer Summe aus zwei Gaußfunktionen gemäß

$$
g(\tilde{\nu}, t)=\sum_{i=1}^{2} g_{0, i}(t) \cdot \exp \left(-0.5\left[\frac{\tilde{\nu}-\tilde{\nu}_{0, i}(t)}{\Delta_{i}(t)}\right]^{2}\right)
$$

angepaßt. Dabei klingt die Amplitude der zweiten Gaußfunktion im Vergleich zur Fluoreszenzlebensdauer rasch auf Null ab, und zwar umso schneller je höher die jeweilige Temperatur ist. Die Anpassung mit zwei Gaußfunktionen könnte mit dem Auftreten zweier fluoreszierender Spezies oberhalb des Hauptphasenüberganges interpretiert werden. Die Messung bei $T=25^{\circ} \mathrm{C}$ stellt einen Grenzfall dar. Sie wurde bis ca. 10 ns mit zwei Gaußfunktionen angepaßt, darüberhinaus mit einer Log-Normal-Funktion. Beispielhaft sind in Abbildung 5.11 typische Anpassungen für $T=5{ }^{\circ} \mathrm{C}$ und $T=60{ }^{\circ} \mathrm{C}$ bei $t=0.58 \mathrm{~ns}$ dargestellt.
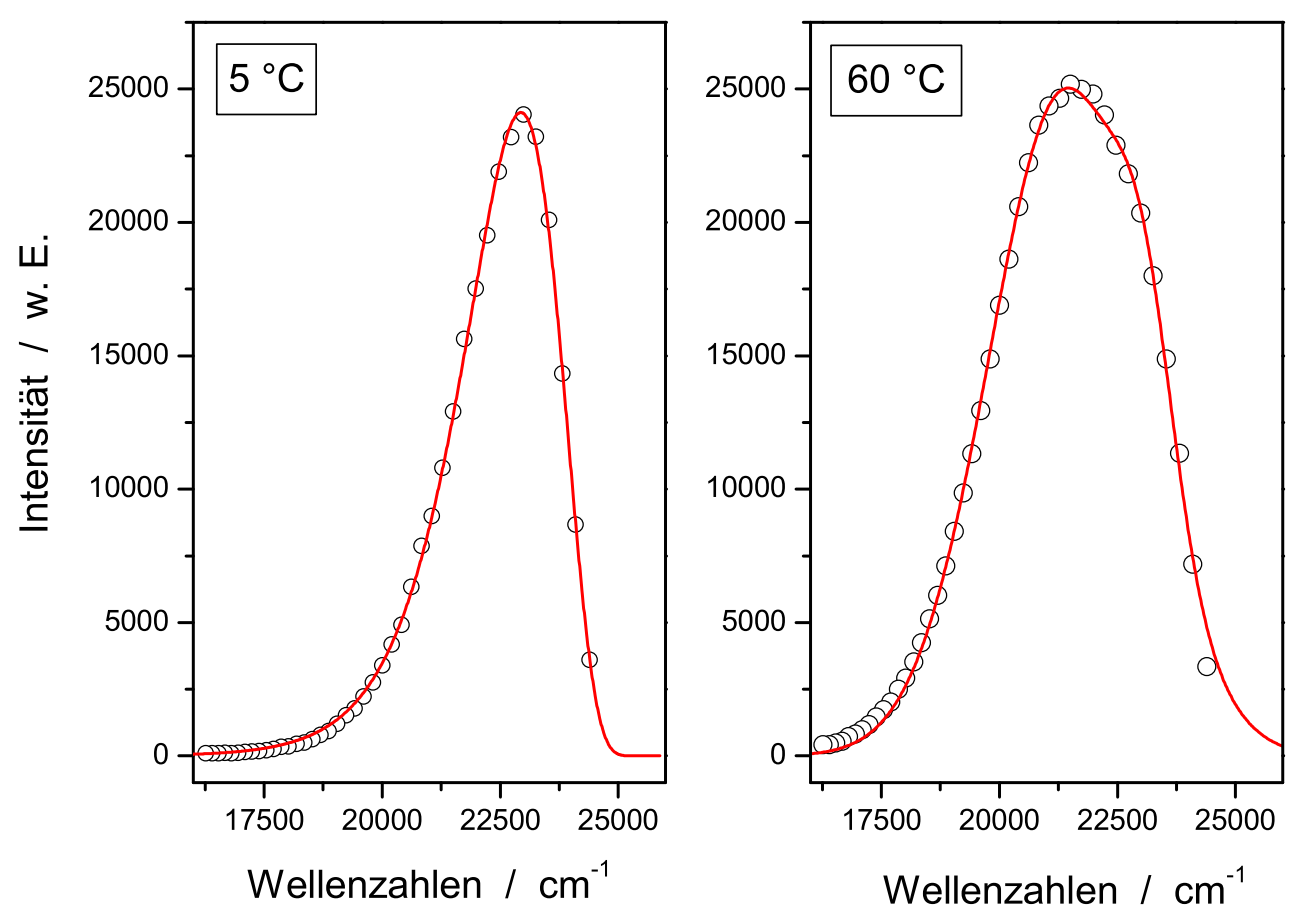

Abbildung 5.11: Beispielhafte Anpassungen von zeitabhängigen Fluoreszenzspektren bei $t=0.58$ ns. Links: Log-Normal-Anpassung für $T=5{ }^{\circ} \mathrm{C}$; Rechts: Anpassung mit einer Summe aus zwei Gaußfunktionen für $T=60^{\circ} \mathrm{C}$. 


\section{Fluoreszenzlebensdauer}

Das Frequenzintegral der Anpassungsfunktion $g(\tilde{\nu}, t)$ als Funktion der Zeit bietet Zugang zur Ermittlung der Fluoreszenzlebensdauer von Laurdan in DMPC in Abhängigkeit der Temperatur:

$$
I_{\mathrm{Fl}, \operatorname{Exp}}(t)=\int_{0}^{\infty} g(\tilde{\nu}, t) \mathrm{d} \tilde{\nu}
$$

Die erhaltenen Kurven $I_{\mathrm{Fl}}$, Exp $(t)$ weisen ein monoexponentielles Abklingverhalten auf, deren Zeitkonstante die Fluoreszenzlebensdauer darstellt:

$$
I_{\mathrm{Fl}, \mathrm{Fit}}(t)=A_{\mathrm{Fl}} \exp \left(-\frac{t}{\tau_{\mathrm{Fl}}}\right)
$$

Die Anpassung der experimentellen Kurven $I_{\mathrm{Fl} \text {, Exp }}(t)$ mit der Anpassungsfunktion $I_{\mathrm{Fl} \text {, Fit }}(t)$ über ein Least-Squares-Verfahren führt zu den in Tabelle 5.6 zusammengefaßten Anpassungsparametern.

\begin{tabular}{|c|c|c|c|}
\hline $\boldsymbol{T} /{ }^{\circ} \mathbf{C}$ & $\boldsymbol{A}_{\mathbf{F l}} / \mathbf{1 0}^{\mathbf{7}}$ counts & $\boldsymbol{\tau}_{\mathbf{F l}} / \mathbf{n s}$ & $\boldsymbol{\tau}_{\boldsymbol{i}} / \mathbf{n s}$ \\
\hline 5 & 7.24 & 6.42 & \\
10 & 8.35 & 6.38 & \\
15 & 9.85 & 6.29 & \\
20 & 8.99 & 6.15 & \\
25 & 9.84 & 5.03 & 24.5 \\
30 & 10.74 & 4.40 & 14.5 \\
35 & 14.50 & 4.12 & 11.8 \\
40 & 14.25 & 3.93 & 10.4 \\
45 & 13.19 & 3.73 & 9.08 \\
50 & 13.51 & 3.51 & 7.88 \\
55 & 13.50 & 3.35 & 7.12 \\
60 & 13.05 & 3.24 & 6.65 \\
\hline
\end{tabular}

Tabelle 5.6: Temperaturabhängige Fluoreszenzlebensdauer und Zeitkonstante des desaktivierenden Kanals von Laurdan in DMPC.

Die experimentell zugängliche Fluoreszenzlebensdauer $\tau_{\mathrm{Fl}}$ eines emittierenden Zustandes setzt sich aus der Strahlungslebensdauer $\tau_{\text {Rad }}$, d.h. der natürlichen Lebensdauer des angeregten Zustandes, sowie allen konkurrierenden Desaktivierungskanälen $i$ mit den Zeitkonstanten $\tau_{i}$ wie folgt zusammen:

$$
\frac{1}{\tau_{\mathrm{Fl}}(T)}=\frac{1}{\tau_{\mathrm{Rad}}}+\sum_{i} \frac{1}{\tau_{i}(T)} .
$$


Dabei ist die natürliche Lebensdauer $\tau_{\text {Rad }}$ eine intrinsische Eigenschaft des untersuchten Chromophors und stellt keine Funktion der Temperatur dar. Desaktivierende Kanäle, wie z. B. Fluoreszenzlöschung (Quenching) durch Stöße mit dem Lösungsmittelbad oder die Bildung von Charge-Transfer-Komplexen, werden in der Summe über alle auftretenden Konkurrenzprozesse erfaßt. Diese können eine Funktion der Temperatur sein, woraus eine Temperaturabhängigkeit der experimentell beobachteten Fluoreszenzlebensdauer resultiert.

Die ermittelten Amplituden $A_{\mathrm{Fl}}$ zeigen, daß die Intensität der abgestrahlten Fluoreszenz unterhalb des Hauptphasenübergangs im Gegensatz zu oberhalb deutlich verringert ist. Das Temperaturverhalten der Fluoreszenzlebensdauer ist in Abbildung 5.12 links in einer Auftragung von $\tau_{\mathrm{Fl}}^{-1}$ gegen die reziproke, absolute Temperatur nach Gleichung 5.13 anschaulich dargestellt. In der gelförmigen und Rippled-Phase erweist sich die Fluoreszenzlebensdauer in erster Näherung als konstant. Unter der Annahme, daß unterhalb des Hauptphasenübergangs desaktivierende Kanäle keine Rolle spielen, läßt sich die Strahlungslebensdauer zu etwa $\tau_{\text {Rad }}=6.33 \mathrm{~ns}$ bestimmen. Oberhalb des Hauptphasenübergangs erscheint die Fluoreszenzlebensdauer dann deutlich verringert, wobei sie mit ansteigender Temperatur immer kürzer wird. Offenbar öffnet sich in der flüssig-kristallinen Phase ein zuvor nicht vorhandener Desaktivierungskanal, der zu einer schnellen
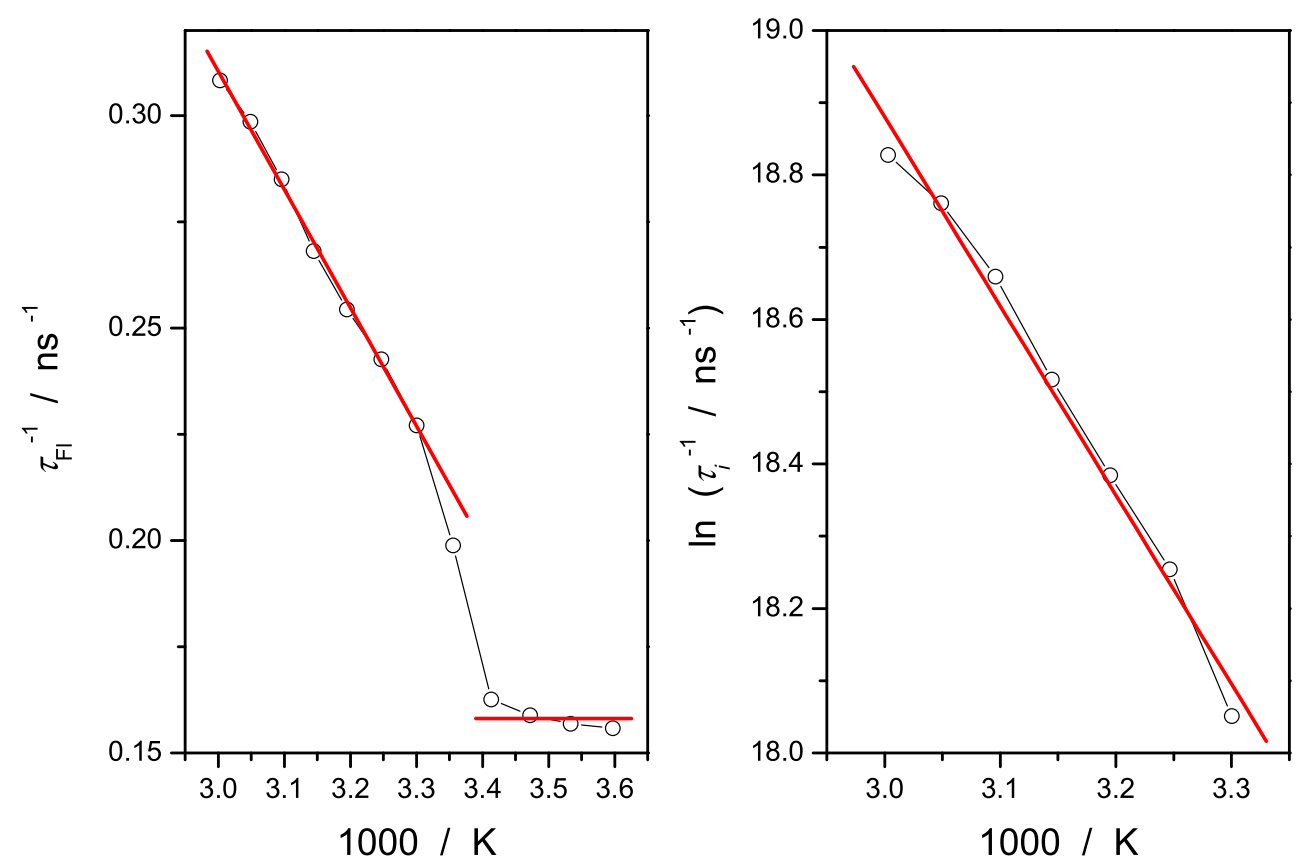

Abbildung 5.12: Links: Temperaturabhängigkeit der Fluoreszenzlebensdauer von Laurdan in DMPC; Rechts: Arrhenius-Auftragung innerhalb der flüssigkristallinen Phase von Laurdan in DMPC. 
Depopulation des lokal angeregten Zustandes führt. Es könnte sich dabei um die Bildung eines Charge-Transfer-Komplexes handeln, wie in der Literatur diskutiert wird $[142,171]$. Dieses Ergebnis steht im Einklang mit der Beobachtung, daß zur Anpassung der zeitabhängigen Fluoreszenzspektren oberhalb des Hauptphasenübergangs zwei Gaußfunktionen herangezogen werden müssen. Mit Hilfe der zuvor ermittelten Strahlungslebensdauer $\tau_{\text {Rad }}$ läßt sich eine temperaturabhängige Lebensdauer $\tau_{i}$ für diesen Kanal bestimmen, welche in Tabelle 5.6 mit aufgeführt ist. Die temperaturabhängige Zeitkonstante $\tau_{i}$ ist in Abbildung 5.12 rechts als Arrhenius-Auftragung $(\ln (1 / \tau)$ gegen $1 / T)$ nach der Arrhenius-Gleichung:

$$
\frac{1}{\tau_{i}(T)}=A_{0} \exp \left(-\frac{E_{\mathrm{a}}}{R T}\right)
$$

dargestellt. Darin bezeichnet $A_{0}$ den präexponentiellen Faktor, $E_{\mathrm{a}}$ die Aktivierungsenergie und $R$ die Gaskonstante. Anhand der dargestellten, linearen Anpassung wurde die Aktivierungsenergie des sich öffnenden Kanals zu $E_{\mathrm{a}}=21.8 \mathrm{~kJ} \mathrm{~mol}^{-1}$ und der präexponentielle Faktor zu $A_{0}=4.0 \cdot 10^{11} \mathrm{~s}^{-1}$ bestimmt.

\section{Zeitentwicklung der Emissionsspektren}

Die Untersuchung der Zeitentwicklung der Emissionsspektren über die Zeitabhängigkeit der verschiedenen Anpassungsparameter erwies sich als problematisch. Zum einen ist es auf diese Weise nicht möglich, die mit unterschiedlichen Formfunktionen angepaßten Spektren untereinander zu vergleichen, zum anderen erwies sich insbesondere die Lage des Maximums der jeweiligen Anpassungen zur Bestimmung des Stokes-Shifts als stark fehlerbehaftet und damit ungeeignet. Dies gilt insbesondere für Spektren die ein Plateau oder gar ein Doppelmaximum aufweisen (siehe z. B. rechtes Spektrum in Abbildung 5.11).

Daher werden die Anpassungsfunktionen $g(\tilde{\nu})$ über ihre statistischen Momente beschrieben, welche einen von der verwendeten analytischen Darstellung unabhängigen Vergleich erlauben und sich als wesentlich geeigneter zur Bestimmung des Stokes-Shifts erwiesen. Sie sind definiert als [239]:

Das $n$-te Moment:

$$
\alpha_{n}=\left\langle\tilde{\nu}^{n}\right\rangle=\frac{\int_{-\infty}^{\infty} \tilde{\nu}^{n} g(\tilde{\nu}) \mathrm{d} \tilde{\nu}}{\int_{-\infty}^{\infty} g(\tilde{\nu}) \mathrm{d} \tilde{\nu}}
$$

Das $n$-te zentrale Moment:

$$
\mu_{n}=\left\langle(\tilde{\nu}-\langle\tilde{\nu}\rangle)^{n}\right\rangle=\frac{\int_{-\infty}^{\infty}(\tilde{\nu}-\langle\tilde{\nu}\rangle)^{n} g(\tilde{\nu}) \mathrm{d} \tilde{\nu}}{\int_{-\infty}^{\infty} g(\tilde{\nu}) \mathrm{d} \tilde{\nu}}
$$


Die Bestimmung der ersten drei Momente oder äquivalent dazu die Bestimmung des ersten Momentes $\langle\tilde{\nu}\rangle$ und des zweiten und dritten zentralen Momentes $\mu_{2}$ bzw. $\mu_{3}$ gibt die wesentlichen Merkmale der Anpassungsfunktionen der zeitaufgelösten Spektren wieder. Dabei entspricht $\langle\tilde{\nu}\rangle$ dem Schwerpunkt der Anpassungsfunktion. $\mu_{2}$ und $\mu_{3}$ beschreiben entsprechend Halbwertsbreite und Asymmetrie. Für die Log-Normal-Funktion können diese Momente analytisch bestimmt werden:

$$
\begin{aligned}
\langle\tilde{\nu}\rangle & =\tilde{\nu}_{0}+\frac{\Delta}{2 b}\left(\exp \left(\frac{3 b^{2}}{4 \ln (2)}\right)-1\right) \\
\mu_{2} & =\left(\frac{\Delta}{2 b}\right)^{2} \exp \left(\frac{3 b^{2}}{2 \ln (2)}\right)\left(\exp \left(\frac{b^{2}}{2 \ln (2)}\right)-1\right) \\
\mu_{3} & =\left(\frac{\Delta}{2 b}\right)^{3} \exp \left(\frac{9 b^{2}}{4 \ln (2)}\right)\left(\exp \left(\frac{3 b^{2}}{2 \ln (2)}\right)-3 \exp \left(\frac{b^{2}}{2 \ln (2)}\right)+2\right) .
\end{aligned}
$$

Für die Anpassungen mit einer Summe aus zwei Gaußfunktionen erfolgte die Berechnung der Momente numerisch. Um die Auftragungen der verschiedenen Momente direkt vergleichen zu können, müssen gleiche Einheiten gewählt werden [240,241]. Daher wird im folgenden stets die Wurzel aus dem zweiten zentralen Moment und die dritte Wurzel aus dem dritten zentralen Moment betrachtet. Zur Vereinfachung wird jedoch im folgenden nur von den Momenten und nicht von den entsprechenden Wurzeln gesprochen. Nachfolgend werden die erhaltenen Momente in Abhängigkeit der Verzögerungszeit nach Anregung der Probe diskutiert.

Schwerpunkt Abbildung 5.13 zeigt das erste Moment, d. h. den Schwerpunkt der angepaßten Spektren, in Abhängigkeit der Zeit nach Anregung. Es handelt sich hierbei also um eine direkte Beobachtung des zeitabhängigen Fluoreszenz Stokes-Shifts. Alle Kurven weisen eine Verschiebung zu geringeren Wellenzahlen im beobachteten Zeitfenster auf, entsprechend der erfolgenden Stabilisierung der durch Laseranregung geschalteten Dipole durch dipolare Solvatation (siehe Kapitel 2.2). Unterhalb des Hauptphasenübergangs ist lediglich eine geringe Verschiebung des Schwerpunktes von wenigen hundert $\mathrm{cm}^{-1} \mathrm{zu}$ geringeren Wellenzahlen zu beobachten, welche mit steigender Temperatur geringfügig zunimmt. Nach Überschreiten des Hauptphasenübergangs vergrößert sich die maximal beobachtbare Schwerpunktsverschiebung deutlich, um schließlich gegen einen Grenzwert bei ca. $20550 \mathrm{~cm}^{-1} \mathrm{zu}$ streben. Der Verlauf kann unterhalb des Hauptphasenübergangs durch eine Summe aus drei, oberhalb durch eine Summe aus zwei Exponentialfunktionen beschrieben werden. 


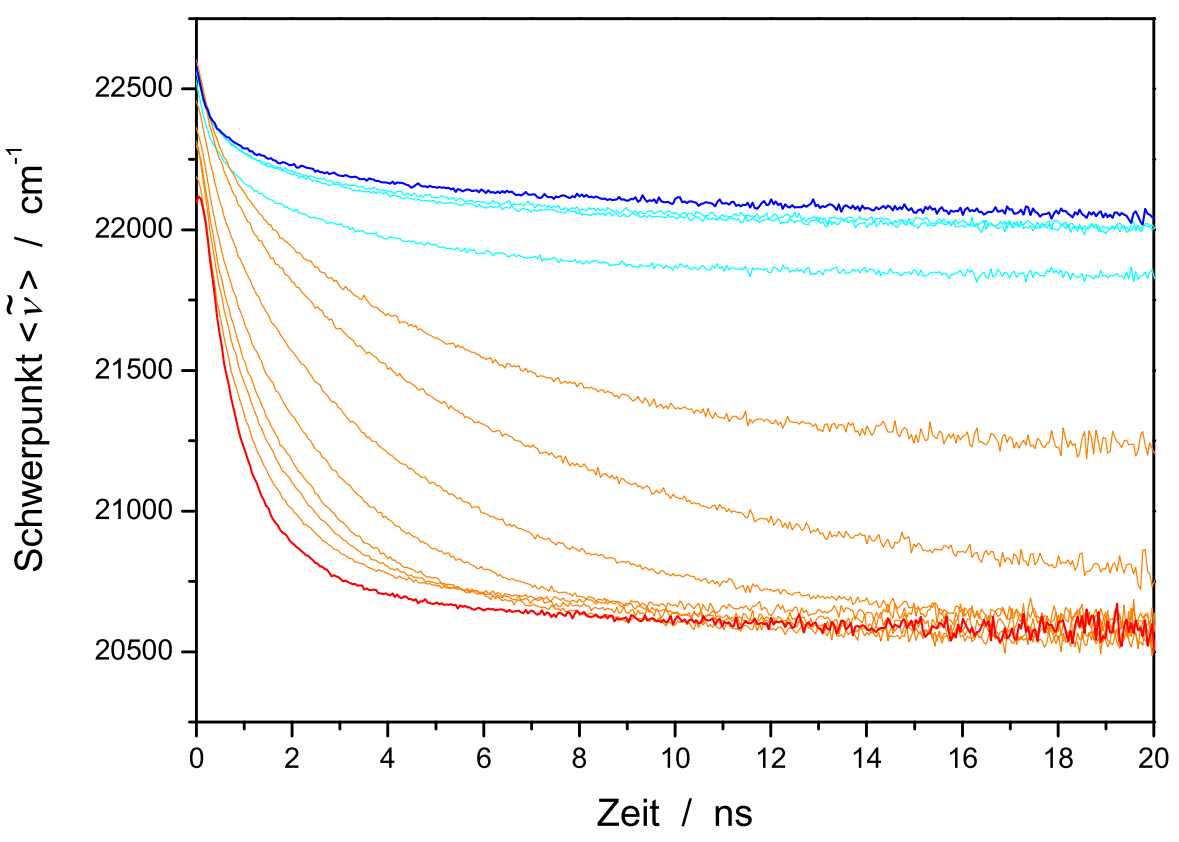

Abbildung 5.13: Darstellung des zeitlichen Verlaufs des Schwerpunktes $\langle\tilde{\nu}\rangle$ der Fluoreszenzspektren von Laurdan in DMPC für $T=5^{\circ} \mathrm{C}$ (blau) bis $T=60^{\circ} \mathrm{C}$ (rot).

Die große Ähnlichkeit des zeitabhängigen Stokes-Shifts (Abbildung 5.13) und der zeitabhängigen Anisotropie (Abbildung 5.7) ist nicht zufällig. Wie in der späteren Diskussion noch gezeigt werden wird, finden bei der Stabilisierung eines erzeugten Nichtgleichgewichtszustandes durch Solvatation die gleichen Mechanismen statt, wie bei der Messung der Rotationsvorgänge des Chromophors über die Anisotropie. Das zeitliche Verhalten des Schwerpunkts in Abhängigkeit der Temperatur läßt sich zunächst vereinfacht wie folgt verstehen: Das Überschreiten des Hauptphasenübergangs führt wie bereits angemerkt durch das Schmelzen der Kettenregion der Membran zu einer erhöhten Wasserkonzentration in der Kopfgruppenregion der Lipidmembran. Außerdem kommt es, wie in den Anisotropiemessungen gezeigt wurde, zu einer erhöhten Membranflexibilität und damit Bewegungsfreiheit des angeregten Chromophors. Diese beiden Effekte führen zu einer effektiveren Stabilisierung des Chromophors in der weniger eingeschränkten Umgebung, was sich in einem stark erhöhten Stokes-Shift innerhalb der flüssigkristallinen Phase ausdrückt. Eine genaue Analyse der zugrundeliegenden Dynamiken erfolgt für die aus diesen Daten abgeleitete Solvatationskorrelationsfunktion in Kapitel 5.4.2. Eine eingehende Diskussion der Effekte folgt in Kapitel 5.5. 
Halbwertsbreite Das zweite zentrale Moment beschreibt die Halbwertsbreite der Anpassungsfunktionen (siehe Abbildung 5.14). Es zeigt sich, daß unterhalb des Hauptphasenübergangs mit fortschreitender Zeit nach Anregung die Halbwertsbreite der Fluoreszenzspektren zunächst sehr schnell (innerhalb der ersten 500 ps) und darauffolgend sehr viel langsamer (im Bereich mehrerer Nanosekunden) zunimmt und gegen einen konstanten Wert strebt. Oberhalb des Hauptphasenübergangs nimmt die Breite nach erfolgter Anregung zunächst zu, und zwar sehr viel stärker als unterhalb des Phasenübergangs. Sie durchläuft dann aber ein Maximum und nimmt anschließend wieder ab, um gegen einen konstanten Wert zu streben, der im gleichen Bereich wie unterhalb des Phasenübergangs liegt. Die Lage des Maximums verschiebt sich mit steigender Temperatur zu geringeren Verzögerungszeiten.

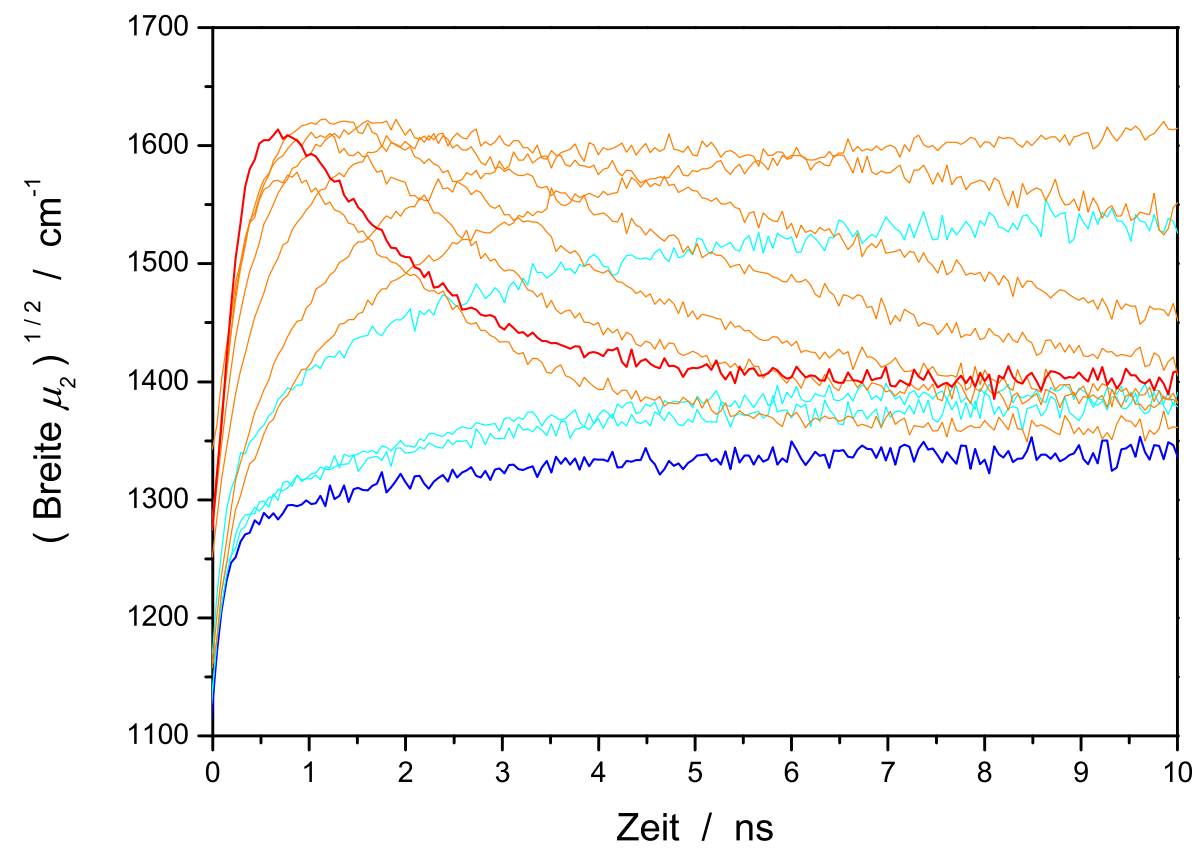

Abbildung 5.14: Darstellung des zeitlichen Verlaufs der Halbwertsbreite $\left(\mu_{2}\right)^{1 / 2}$ der Fluoreszenzspektren von Laurdan in DMPC für $T=5^{\circ} \mathrm{C}$ (blau) bis $\mathrm{T}=60^{\circ} \mathrm{C}$ (rot).

Das beobachtete Verhalten für die zeitliche Entwicklung der Halbwertsbreite der Spektren wurde bereits in der Literatur ausführlich beschrieben [135, 139, 242]. Wenn die Solvatation innerhalb der Fluoreszenzlebensdauer der angeregten Farbstoffsonde abgeschlossen ist und ein Großteil der Solvatation mit der gegebenen Zeitauflösung erfaßt werden kann, so vergrößert sich die beobachtete Halbwertsbreite zunächst und durchläuft dann ein Maximum bei einer Zeit, die in etwa der mittleren Solvatationszeit entspricht. Anschließend nimmt die Breite ab und 
strebt gegen einen Grenzwert. Wenn die Solvatation über einen längeren Zeitraum erfolgt, als der angeregte Chromophor fluoresziert, wird lediglich ein Ansteigen der Breite beobachtet. Entsprechend würde kein Ansteigen beobachtet werden, wenn die Solvatation im Mittel schneller als die gegebene Zeitauflösung ist.

Mit diesen Richtlinien läßt sich feststellen, daß die Solvation innerhalb der gelförmigen und Rippled-Phase noch nicht vollständig innerhalb der Fluoreszenzlebensdauer des angeregten Zustandes von ca. 6 ns abgeschlossen ist. Dagegen werden für die flüssig-kristalline Phase Maxima der Halbwertsbreite durchlaufen, was darauf hinweist, daß die mittleren Solvatationszeiten im Bereich der Fluoreszenzlebensdauer liegen. Das Maximum verschiebt sich dabei mit steigender Temperatur zu kürzen Zeiten was einer schneller werdenden Solvatation in dieser Phase entspricht. Außerdem zeigt sich, daß mit der Methode des zeitkorrelierten Einzelphotonenzählens ein relevanter Teil der Solvatationsdynamik erfaßt werden kann. Diese Beobachtungen bedeuten aber nicht, daß nicht auch schnellere Zeitkomponenten an der Solvatation teilhaben, die mit der Technik des zeitkorrelierten Einzelphotonenzählens nicht aufgelöst werden können. Eine genauere Analyse dieses Sachverhalts wird im folgenden Kapitel anhand der Solvatationskorrelationsfunktion durchgeführt.

Asymmetrie Die Asymmetrien der Anpassungsfunktionen werden durch das dritte zentrale Moment beschrieben und sind in Abbildung 5.15 dargestellt. Die ermittelten Werte sind durchweg negativ. Unterhalb des Hauptphasenübergangs wächst die beobachtete Asymmetrie zunächst sehr schnell an und strebt dann auf einer Nanosekunden-Zeitskala gegen einen konstanten Wert. Mit steigender Temperatur nimmt die Asymmetrie geringfügig zu, zeigt aber ein ähnliches Verhalten. Oberhalb des Hauptphasenübergangs weisen die Spektren generell eine geringere Asymmetrie auf, was auf eine homogenere Umgebung des Chromophors innerhalb der flüssig-kristallinen Phase hinweist. Die Asymmetrie durchläuft in Abhängigkeit der Zeit zunächst ein schwach ausgeprägtes Maximum um 500 ps und nimmt dann mit fortschreitender Zeit nach Anregung ab, um schließlich gegen Null zu streben. Mit steigender Temperatur wird die Abnahme der Asymmetrie dabei zusehends schneller. Dieser Effekt ist mit dem schnelleren Abklingen der Amplitude der zur Anpassung verwendeten zweiten Gaußfunktion mit steigender Temperatur zu erklären, was wiederum auf eine homogener werdende Umgebung hinweist. Im Grenzfall einer Anpassung mit nur einer Gaußfunktion ist die Asymmetrie dann Null. Insgesamt gesehen ähnelt das Verhalten der Asymmetrie damit der zeitlichen Entwicklung der Breite. 


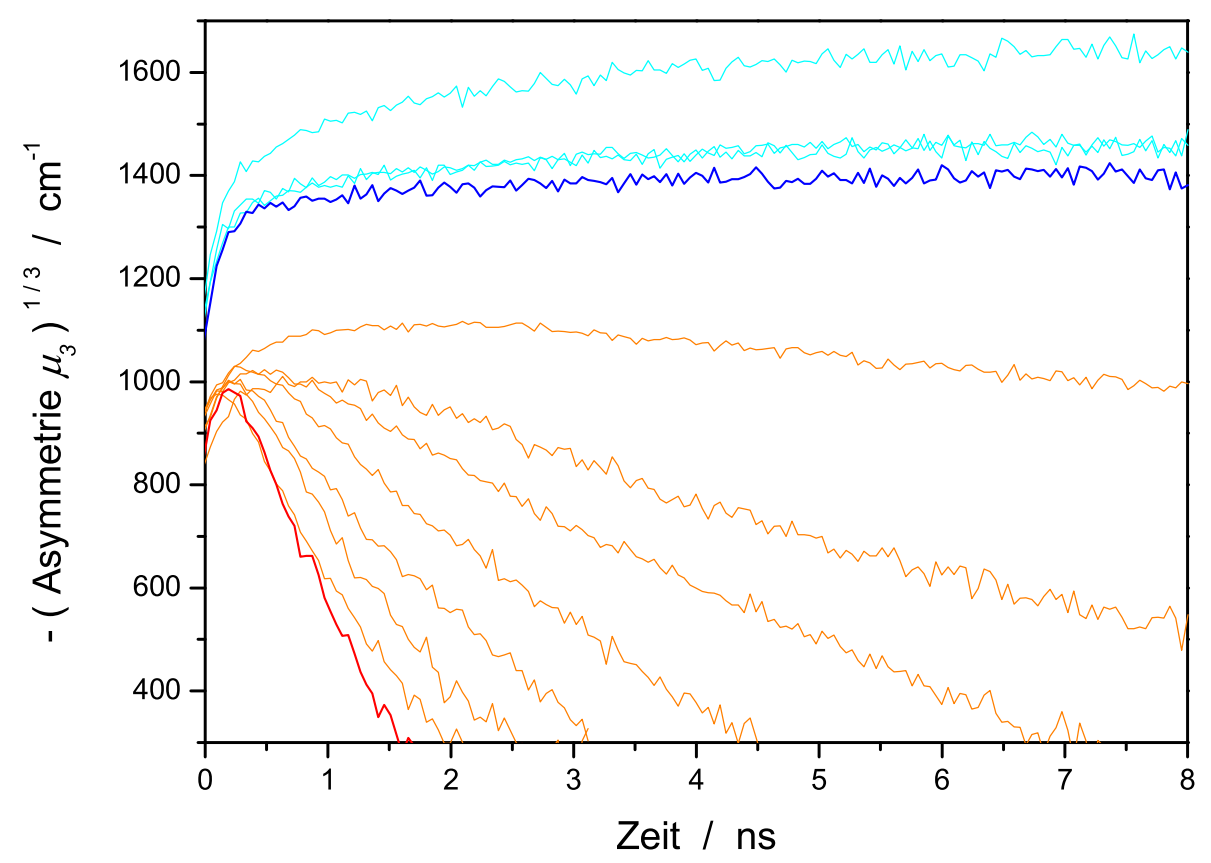

Abbildung 5.15: Darstellung des zeitlichen Verlaufs der Asymmetrie der Fluoreszenzspektren von Laurdan in DMPC für $T=5^{\circ} \mathrm{C}$ (blau) bis $\mathrm{T}=60^{\circ} \mathrm{C}$ (rot).

\subsubsection{Solvatation an der Lipidmembran/Wasser- Grenzschicht}

Um die zugrundeliegende Solvatationsdynamik im untersuchten System zu bestimmen, muß die Solvatationskorrelationsfunktion $S(t)$ gemäß Gleichung 2.1 aufgestellt werden. Wird als Maß für $\Delta E$ stets der Schwerpunkt betrachtet, so läßt sich die Solvatationskorrelationsfunktion in folgender Form ausdrücken:

$$
S_{\langle\tilde{\nu}\rangle}(t)=\frac{\langle\tilde{\nu}\rangle(t)-\langle\tilde{\nu}\rangle(t=\infty)}{\langle\tilde{\nu}\rangle(t=0)-\langle\tilde{\nu}\rangle(t=\infty)} .
$$

Dabei wird $\langle\tilde{\nu}\rangle(t)$, wie im vorhergehenden Kapitel beschrieben, aus dem ersten Moment der Anpassungsfunktionen der zeitabhängigen Fluoreszenzspektren gewonnen. Die Bestimmung von $\langle\tilde{\nu}\rangle(t=0)$ und $\langle\tilde{\nu}\rangle(t=\infty)$ wird nachfolgend behandelt. 


\section{Bestimmung von $\langle\tilde{\nu}\rangle(t=0)$}

Die meist verwendete Bestimmung von $\langle\tilde{\nu}\rangle(t=0)$ durch Extrapolation der gemessenen Funktion $\langle\tilde{\nu}\rangle(t)$ auf den Zeitnullpunkt $t=0$ hat den schwerwiegenden Nachteil, daß die auf diese Weise bestimmte Solvatationskorrelationsfunktion stark von der zugrundeliegenden Zeitauflösung der verwendeten Meßtechnik abhängt. Insbesondere in Lösungsmitteln, wie z. B. Wasser, die für ihre schnelle Solvatationsdynamik bekannt sind, ist es daher nicht möglich, mit Techniken wie dem zeitkorrelierten Einzelphotonenzählen eine adäquate Korrelationsfunktion zu erhalten, da der frühe Anteil der Solvatationsdynamik nicht auflösbar ist. Ein mit einer fiktiven Technik beliebig kurzer Zeitauflösung bestimmtes Zeitnullpunktsspektrum wäre ebenso wenig zur Aufstellung einer sinnvollen Korrelationsfunktion geeignet, da in diesem Falle zusätzlich zur Solvatationsdynamik auch die gesamte intramolekulare Schwingungsrelaxation von Überschußenergie in den Franck-Condon aktiven Moden aufgelöst werden würde. Gesucht ist daher das Spektrum, das sich ergibt, nachdem die gesamte intramolekulare Dynamik des Chromophors abgeschlossen ist, aber noch keine Solvatation durch das Lösungsmittel stattgefunden hat.

Ein adäquates Zeitnullpunktsspektrum läßt sich nach Fee und Maroncelli mit einer Genauigkeit von besser als $\pm 10 \%$ über die stationären Absorptions- und Emissionsspektren in einem unpolaren Lösungsmittel bestimmen [26]. In solchen Lösungsmitteln findet in erster Näherung keine Solvation statt. Der beobachtete Shift resultiert dann lediglich aus der Überschußenergie in den Franck-Condon aktiven Moden und den vibronischen Besetzungsverhältnissen des Chromophors. Die in einem unpolaren Lösungsmittel beobachteten Spektren eignen sich somit zur Bestimmung des korrekten Zeitnullpunktsspektrums. Es wurden daher temperaturabhängige Absorptions- und Emissionsspektren des verwendeten Farbstoffes Laurdan in $n$-Hexan aufgenommen (siehe Abbildung 5.1 für $T=25^{\circ} \mathrm{C}$, Spektren für weitere Temperaturen nicht abgebildet). Anschließend konnten aus diesen Spektren und den Absorptionsspektren von Laurdan in der Membran (siehe Abbildung 5.3) die gesuchten temperaturabhängigen Zeitnullpunktsspektren nach der Methode von Fee und Maroncelli berechnet werden [26].

Es wird angenommen, daß sich die stationären Spektren individueller Moleküle in verschiedenen Umgebungen lediglich durch eine allgemeine Energieverschiebung unterscheiden. Die gleiche Linienformfunktion $g(\tilde{\nu})$ beschreibt also die Absorptionsspektren aller Moleküle bis auf eine umgebungsabhängige Energiedifferenz $\delta$. In einem polaren Medium ist das inhomogen verbreiterte Absorptionsspektrum daher gegeben als:

$$
A_{\mathrm{p}}(\tilde{\nu}) \propto \int g(\tilde{\nu}-\delta) p(\delta) \mathrm{d} \delta
$$

Darin beschreibt die Verteilungsfunktion $p(\delta)$ die Häufigkeit der Moleküle zu einer gegebenen Energieverschiebung, d. h. deren spektralen Shift. Wenn $f(\tilde{\nu})$ die ent- 
sprechende Linienformfunktion der Emission eines einzelnen Moleküls bezeichnet, so läßt sich das Emissionsspektrum berechnen, das sich unmittelbar nach Anregung mit $\tilde{\nu}_{\text {Exc }}$ ergibt [26]:

$$
F_{\mathrm{p}}\left(\tilde{\nu}, t=0 ; \tilde{\nu}_{\mathrm{Exc}}\right) \propto \tilde{\nu}^{3} \tilde{\nu}_{\mathrm{Exc}} \int g\left(\tilde{\nu}_{\mathrm{Exc}}-\delta\right) p(\delta) f(\tilde{\nu}-\delta) k_{\mathrm{Rad}}(\delta) \mathrm{d} \delta
$$

Darin bezeichnet $k_{\text {Rad }}$ die Strahlungszeitkonstante. Die Gleichung beschreibt das Fluoreszenzspektrum, das beobachtet wird, bevor eine Bewegung des Lösungsmittels erfolgt, aber nachdem ein intramolekulares Gleichgewicht eingestellt ist, als eine Faltung der aus der Anregung resultierenden Verteilung $g\left(\tilde{\nu}_{\mathrm{Exc}}-\delta\right) p(\delta)$ mit der Fluoreszenzintensitätsfunktion $f(\tilde{\nu}-\delta) k_{\text {Rad }}(\delta)$. Für eine Berechnung der Zeitnullpunktsspektren werden die Funktionen $g(\tilde{\nu}), f(\tilde{\nu}), p(\delta)$ und $k_{\text {Rad }}$ benötigt.

Die Linienformfunktionen $g(\tilde{\nu})$ und $f(\tilde{\nu})$ lassen sich direkt aus den Absorptionsbzw. Fluoreszenzspektren $A_{\text {np }}(\tilde{\nu})$ bzw. $F_{\text {np }}(\tilde{\nu})$ in einem unpolaren Lösungsmittel erhalten:

$$
\begin{aligned}
& g(\tilde{\nu}) \propto \tilde{\nu}^{-1} A_{\mathrm{np}}(\tilde{\nu}) \quad \text { bzw. } \\
& f(\tilde{\nu}) \propto \tilde{\nu}^{-3} F_{\mathrm{np}}(\tilde{\nu}) .
\end{aligned}
$$

Durch Verwendung dieser Gleichungen wird vorausgesetzt, daß die in unpolaren Lösungsmitteln erhaltenen Spektren ausschließlich homogen verbreitert sind und diese Homogenität in allen unpolaren Lösungsmitteln gleich ist. Die Strahlungszeitkonstante $k_{\mathrm{Rad}}$ wird möglichst einfach berechnet nach:

$$
k_{\operatorname{Rad}}(\delta) \propto \frac{\int f(\tilde{\nu}-\delta) \tilde{\nu}^{3} \mathrm{~d} \tilde{\nu}}{\int f(\tilde{\nu}-\delta) \mathrm{d} \tilde{\nu}} .
$$

Schließlich wird die Verteilungsfunktion $p(\delta)$ als gaußförmig angenommen:

$$
p(\delta)=\frac{1}{\sqrt{2 \pi \sigma^{2}}} \exp \left(-0.5\left(\frac{\delta-\delta_{0}}{\sigma}\right)^{2}\right),
$$

wobei $\delta_{0}$ den mittleren durch das polare Lösungsmittel erzeugten Shift und $\sigma$ die Breite der Verteilung bezeichnet. Die Berechnung der Zeitnullpunktsspektren erfolgt dann wie folgt: Zunächst werden $g(\tilde{\nu})$ und $f(\tilde{\nu})$ aus den Absorptions- und Fluoreszenzspektren des Farbstoffes Laurdan in $n$-Hexan gemäß Gleichung 5.23 und 5.24 erhalten. Durch bestmögliche Anpassung der gemessen Spektren $A_{\mathrm{p}}(\tilde{\nu})$ von Laurdan in DMPC nach Gleichung 5.21 werden die Anpassungsparameter $\delta_{0}$ und $\sigma$ der Verteilungsfunktion $p(\delta)$ erhalten. Schließlich kann nach Gleichung 5.22 das Zeitnullpunktsspektrum in einer polaren Lösungsmittelumgebung erhalten werden. Die Zeitnullpunktsspektren wurden für Temperaturen von $T=5$ bis $60^{\circ} \mathrm{C}$ in Schritten von $5{ }^{\circ} \mathrm{C}$ unter Verwendung der jeweiligen temperaturabhängigen Spektren $A_{\mathrm{np}}(\tilde{\nu}), F_{\mathrm{np}}(\tilde{\nu})$ und $A_{\mathrm{p}}(\tilde{\nu})$ berechnet. Aus dem erhaltenen Zeitnullpunktsspektrum $F_{\mathrm{p}}(\tilde{\nu})$ wird anschließend der Schwerpunkt $\langle\tilde{\nu}\rangle(t=0)$ bestimmt. Die so ermittelten Schwerpunkte sind in Tabelle 5.7 in Abhängigkeit der Temperatur aufgeführt. 


\section{Bestimmung von $\langle\tilde{\nu}\rangle(t=\infty)$}

Die korrekte Bestimmung der Zeitunendlichspektren, d. h. den Spektren zu großen Zeiten $(t \rightarrow \infty)$, ist ein weiteres Problem bei der Aufstellung der Solvatationskorrelationsfunktion. Für schnell solvatisierende Systeme stellt das stationäre Emissionsspektrum das korrekte Zeitunendlichspektrum dar. Im hier auftretenden Fall einer langsamen Solvatationsdynamik, die sich bis in den Bereich der Fluoreszenzlebensdauer erstreckt, ist es jedoch nicht uneingeschränkt zur Bestimmung von $\langle\tilde{\nu}\rangle(t=\infty)$ geeignet. Wie in Abbildung 5.16 beispielhaft für $T=5{ }^{\circ} \mathrm{C}, T=35^{\circ} \mathrm{C}$ und $T=60^{\circ} \mathrm{C}$ gezeigt, repräsentiert das stationäre Emissionsspektrum nämlich nicht die Verhältnisse zu langen Verzögerungszeiten, sondern stellt vielmehr eine zeitliche Integration über die zeitaufgelösten Fluoreszenzspektren, d. h. über die experimentellen Kinetiken $K(t, \tilde{\nu})$ als Funktion der spektralen Lage dar:

$$
F_{\text {Int }}(\tilde{\nu})=\int_{0}^{\infty} K(t, \tilde{\nu}) \mathrm{d} t
$$
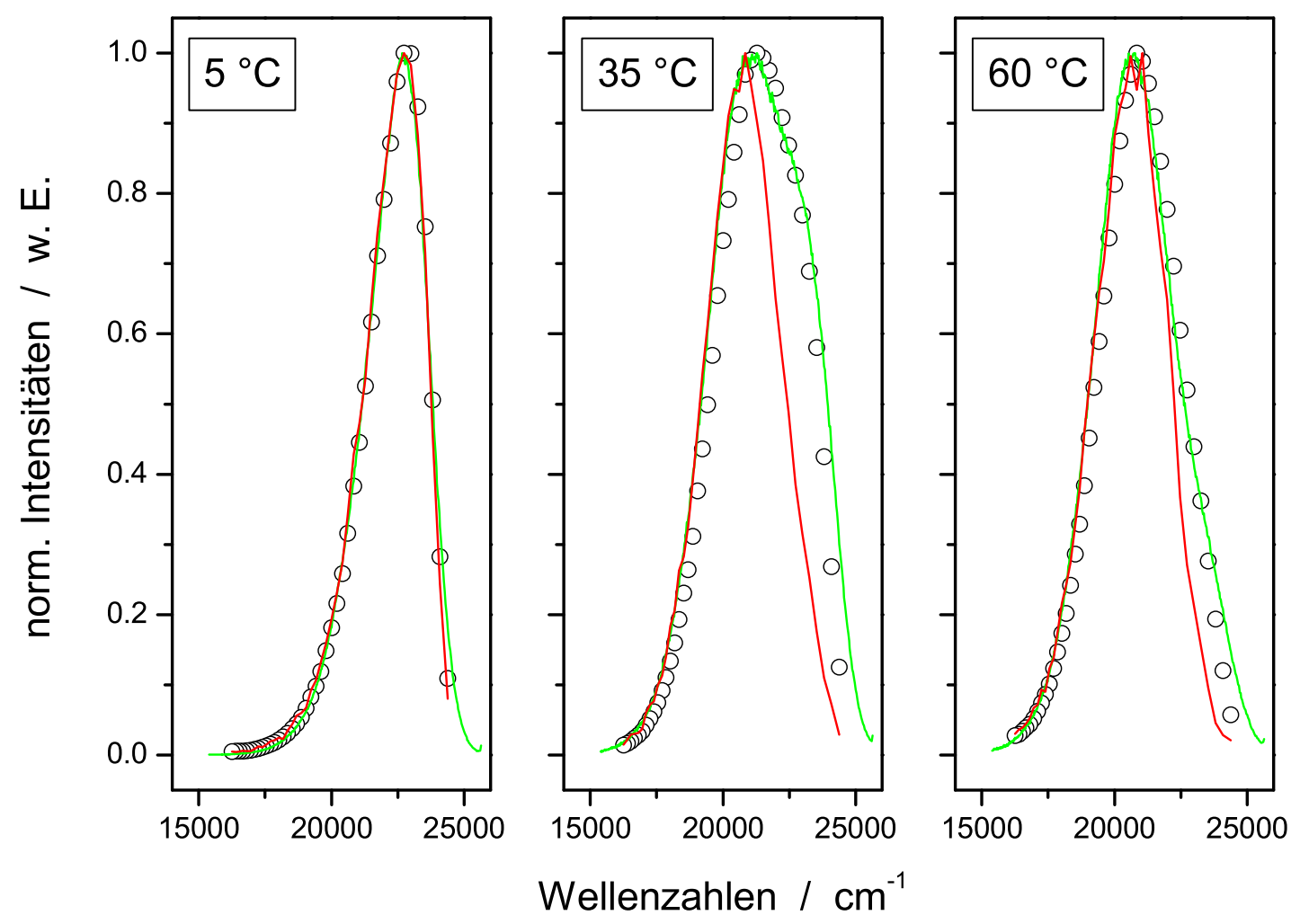

Abbildung 5.16: Gegenüberstellung der zeitlich integrierten Fluoreszenzspektren (schwarze Kreise), der stationären Emissionsspektren (grün) und Fluoreszenzspektren zu langen Verzögerungszeiten (rot) bei $\mathrm{T}=5{ }^{\circ} \mathrm{C}, \mathrm{T}=35^{\circ} \mathrm{C}$ und $\mathrm{T}=60^{\circ} \mathrm{C}$. 
Es gibt für alle Temperaturen eine gute Übereinstimmung zwischen den aufintegrierten Kinetiken (schwarze Kreise) und den stationären Emissionsspektren (grün). Aus dem zeitkorrelierten Einzelphotonenzählexperiment erhaltene zeitaufgelöste Spektren für lange Verzögerungszeiten (rot) zeigen aber insbesondere um den Hauptphasenübergang deutliche Abweichungen vom stationären Emissionsspektrum auf der blauen Flanke. Die stationären Emissionsspektren erweisen sich also zur Bestimmung des Zeitunendlichspektrums als nicht geeignet. Sie würden die Größe des tatsächlich stattfindenden Stokes-Shifts erheblich unterschätzen.

Daher wurde, der Arbeit von Maroncelli und Fleming folgend, das Zeitunendlichspektrum durch „sinnvolle“ Extrapolation der zeitaufgelösten Spektren zu unendlichen Zeiten gewonnen [238]. Die zeitabhängigen ersten Momente der Fluoreszenzspektren wurden zu diesem Zweck unterhalb des Hauptphasenübergangs mit einer Summe aus drei, darüber mit zwei Exponentialfunktionen über ein LeastSquares-Verfahren angepaßt und der Grenzwert für unendliche Zeiten bestimmt. Als problematisch erwies sich dabei, daß der Zeitbereich der Solvatationsdynamik in der Größenordnung der Fluoreszenzlebensdauer des Laurdans in der Membran liegt und die Bestimmung des Grenzwertes daher unter Umständen stark fehlerbehaftet ist. Daher wurde nach Bedarf eine manuelle Korrektur entsprechend der Vorgehensweise von Maroncelli und Fleming vorgenommen [238].

\begin{tabular}{|c|c|c|c|}
\hline $\boldsymbol{T} /{ }^{\circ} \mathbf{C}$ & $\begin{array}{c}\langle\tilde{\boldsymbol{\nu}}\rangle(\boldsymbol{t}=\mathbf{0}) \\
/ \mathbf{c m}^{-\mathbf{1}}\end{array}$ & $\begin{array}{c}\langle\tilde{\boldsymbol{\nu}}\rangle(\boldsymbol{t}=\boldsymbol{\infty}) \\
/ \mathbf{c m}^{-\mathbf{1}}\end{array}$ & $\begin{array}{c}\langle\tilde{\boldsymbol{\nu}}\rangle(\boldsymbol{t}=\mathbf{0})-\langle\tilde{\boldsymbol{\nu}}\rangle(\boldsymbol{t}=\boldsymbol{\infty}) \\
/ \mathbf{c m}^{-\mathbf{1}}\end{array}$ \\
\hline 5 & 24826 & 22060 & 2766 \\
10 & 24846 & 22017 & 2829 \\
15 & 24870 & 21996 & 2874 \\
20 & 24897 & 21820 & 3077 \\
25 & 24923 & 21209 & 3714 \\
30 & 24931 & 20714 & 4217 \\
35 & 24943 & 20588 & 4355 \\
40 & 24954 & 20547 & 4407 \\
45 & 24964 & 20544 & 4420 \\
50 & 24978 & 20592 & 4386 \\
55 & 24990 & 20624 & 4366 \\
60 & 24998 & 20589 & 4409 \\
\hline
\end{tabular}

Tabelle 5.7: Ermittelte Schwerpunkte der Zeitnullpunkts- und Zeitunendlichspektren, sowie resultierender Stokes-Shift. 
Die erhaltenen Grenzwerte $\langle\tilde{\nu}\rangle(t=\infty)$ für die verschiedenen Temperaturen sind ebenfalls in Tabelle 5.7 aufgeführt. Die Differenz $\langle\tilde{\nu}\rangle(t=0)-\langle\tilde{\nu}\rangle(t=\infty)$ stellt dabei den maximal im Experiment beobachtbaren, aus dipolarer Solvatation herrührenden, Stokes-Shift dar.

\section{Solvatationskorrelationsfunktion}

Mit den ermittelten Zeitnullpunkts- und Zeitunendlichspektren kann nun gemäß Gleichung 5.20 die Solvatationskorrelationsfunktion aufgestellt werden. Die erhaltenen Funktionen sind in Abbildung 5.17 dargestellt.

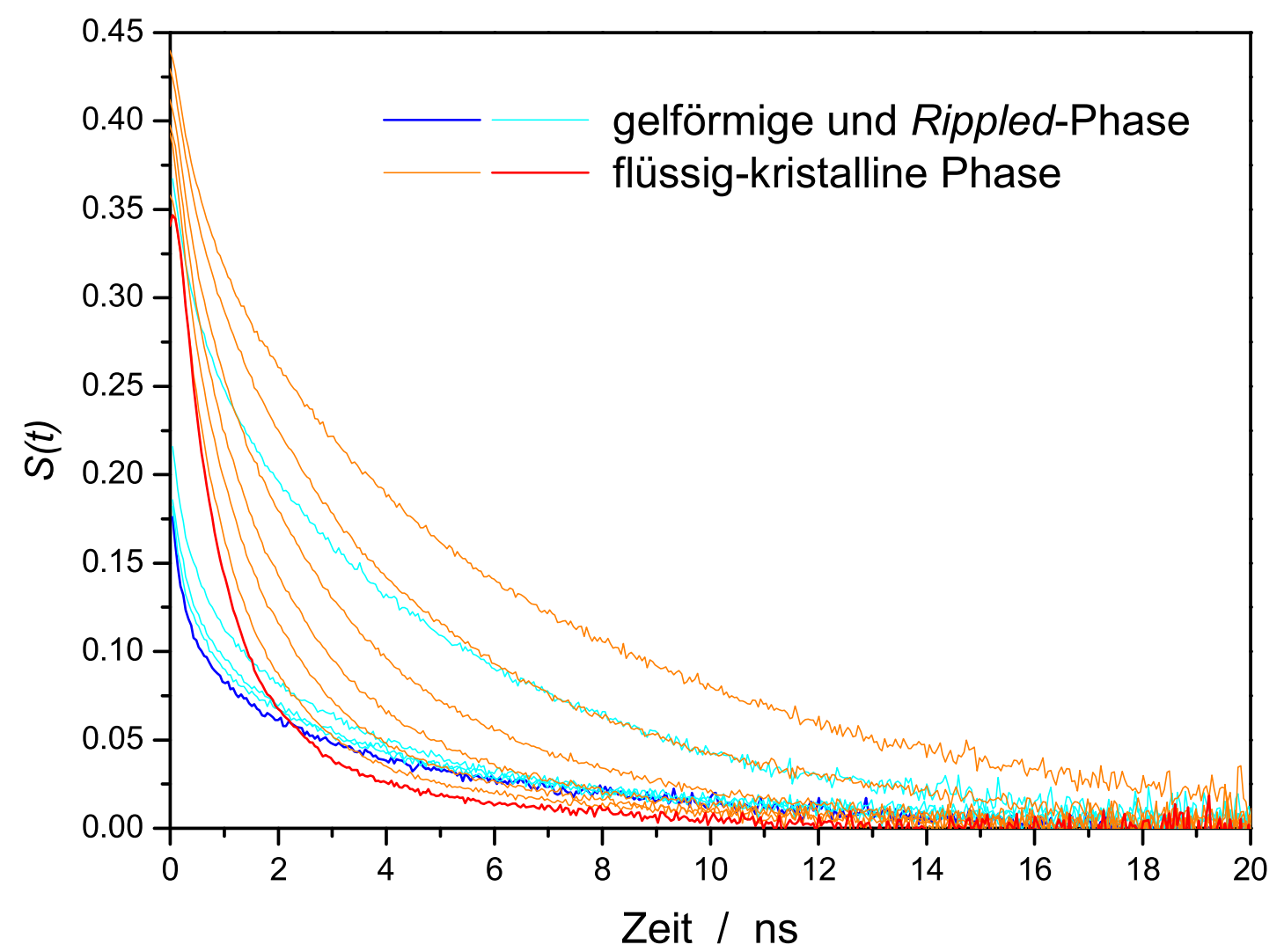

Abbildung 5.17: Mit dem TCSPC-Experiment ermittelte Solvatationskorrelationsfunktion von Laurdan in Vesikeln für $T=5{ }^{\circ} \mathrm{C}$ (blau) bis $T=60^{\circ} \mathrm{C}$ (rot). 
Es zeigt sich, daß das Solvatationsverhalten unterhalb und oberhalb des Hauptphasenübergangs deutlich verschieden ist, wie durch einen Vergleich der blauen und roten Kurven in Abbildung 5.17 leicht ersichtlich ist. Die ausgeprägte Temperaturabhängigkeit der Solvatation ist dabei vorrangig um den Hauptphasenübergang zu erkennen. Zur Untersuchung der zugrundeliegenden Dynamiken wurde die Funktion in der gelförmigen und Rippled-Phase mit einer Summe aus drei, in der flüssig-kristallinen Phase aus zwei Exponentialfunktionen angepaßt:

$$
S(t)=\sum_{i=1}^{2 \text { bzw. } 3} A_{i} \exp \left(-\frac{t}{\tau_{i}}\right) .
$$

Die verwendeten Anpassungsparameter sind in Tabelle 5.8 aufgeführt. In Abbildung 5.18 folgt eine graphische Darstellung der ermittelten Zeitkonstanten $\tau_{i}$ und der Amplituden $A_{i}$.

\begin{tabular}{|c|c|c|c|c|c|c|c|c|}
\hline $\boldsymbol{T} /{ }^{\circ} \mathbf{C}$ & $\boldsymbol{A}_{\mathbf{1}}$ & $\boldsymbol{\tau}_{\mathbf{1}} / \mathbf{n s}$ & $\boldsymbol{A}_{\mathbf{2}}$ & $\boldsymbol{\tau}_{\mathbf{2}} / \mathbf{n s}$ & $\boldsymbol{A}_{\mathbf{3}}$ & $\boldsymbol{\tau}_{\mathbf{3}} / \mathbf{n s}$ & $\langle\boldsymbol{\tau}\rangle / \mathbf{n s}$ & $\boldsymbol{S}(\boldsymbol{t}=\mathbf{0})$ \\
\hline 5 & 0.061 & 0.165 & 0.052 & 0.854 & 0.080 & 5.645 & 0.505 & 0.193 \\
10 & 0.054 & 0.179 & 0.054 & 0.856 & 0.088 & 5.444 & 0.538 & 0.197 \\
15 & 0.065 & 0.285 & 0.064 & 1.619 & 0.068 & 6.939 & 0.597 & 0.198 \\
20 & 0.081 & 0.344 & 0.112 & 2.846 & 0.032 & 10.79 & 0.689 & 0.225 \\
25 & 0.094 & 0.535 & 0.281 & 5.348 & & & 1.553 & 0.375 \\
30 & 0.103 & 0.701 & 0.339 & 6.865 & & & 2.398 & 0.442 \\
35 & 0.112 & 0.832 & 0.318 & 4.966 & & & 1.673 & 0.430 \\
40 & 0.146 & 0.921 & 0.269 & 3.858 & & & 1.172 & 0.415 \\
45 & 0.221 & 1.079 & 0.181 & 3.717 & & & 0.913 & 0.403 \\
50 & 0.264 & 1.006 & 0.131 & 3.719 & & & 0.754 & 0.395 \\
55 & 0.292 & 0.929 & 0.083 & 4.046 & & & 0.607 & 0.375 \\
60 & 0.313 & 0.794 & 0.074 & 3.566 & & & 0.513 & 0.387 \\
\hline
\end{tabular}

Tabelle 5.8: Anpassungsparameter der mit dem TCSPC-Experiment erhaltenen Solvatationskorrelationsfunktion, mittlere Solvatationszeit und experimentelles Zeitnullpunktsspektrum.

Unterhalb des Hauptphasenübergangs wurde die Solvatationskorrelationsfunktion mit einer Summe aus drei Exponentialfunktionen angepaßt, wobei die angepaßten Zeiten $\tau_{i}$ mit steigender Temperatur allesamt größer werden. Die Amplituden der beiden schnelleren Zeitkonstanten wachsen dabei tendenziell leicht an, während die Amplitude der langsamen Zeitkonstante mit steigender Temperatur abnimmt und oberhalb des Hauptphasenübergangs gar keine Relevanz mehr besitzt. Oberhalb des Phasenübergangs zeigt sich ein unterschiedliches Verhalten. Die schnelle Zeitkonstante steigt in geringem Maße bis $T=45^{\circ} \mathrm{C}$ weiter 
an und fällt dann geringfügig ab. Die zugehörige Amplitude wächst jedoch mit steigender Temperatur deutlich an, so daß diese Komponente den Solvatationsprozeß bei hohen Temperaturen dominiert. Die mittlere Zeitkonstante dominiert die Solvatation zwischen $T=25$ und $40^{\circ} \mathrm{C}$. Hier durchlaufen sowohl die Zeitkonstante als auch die zugehörige Amplitude ein Maximum, um schließlich für höhere Temperaturen wieder an Dominanz zu verlieren.
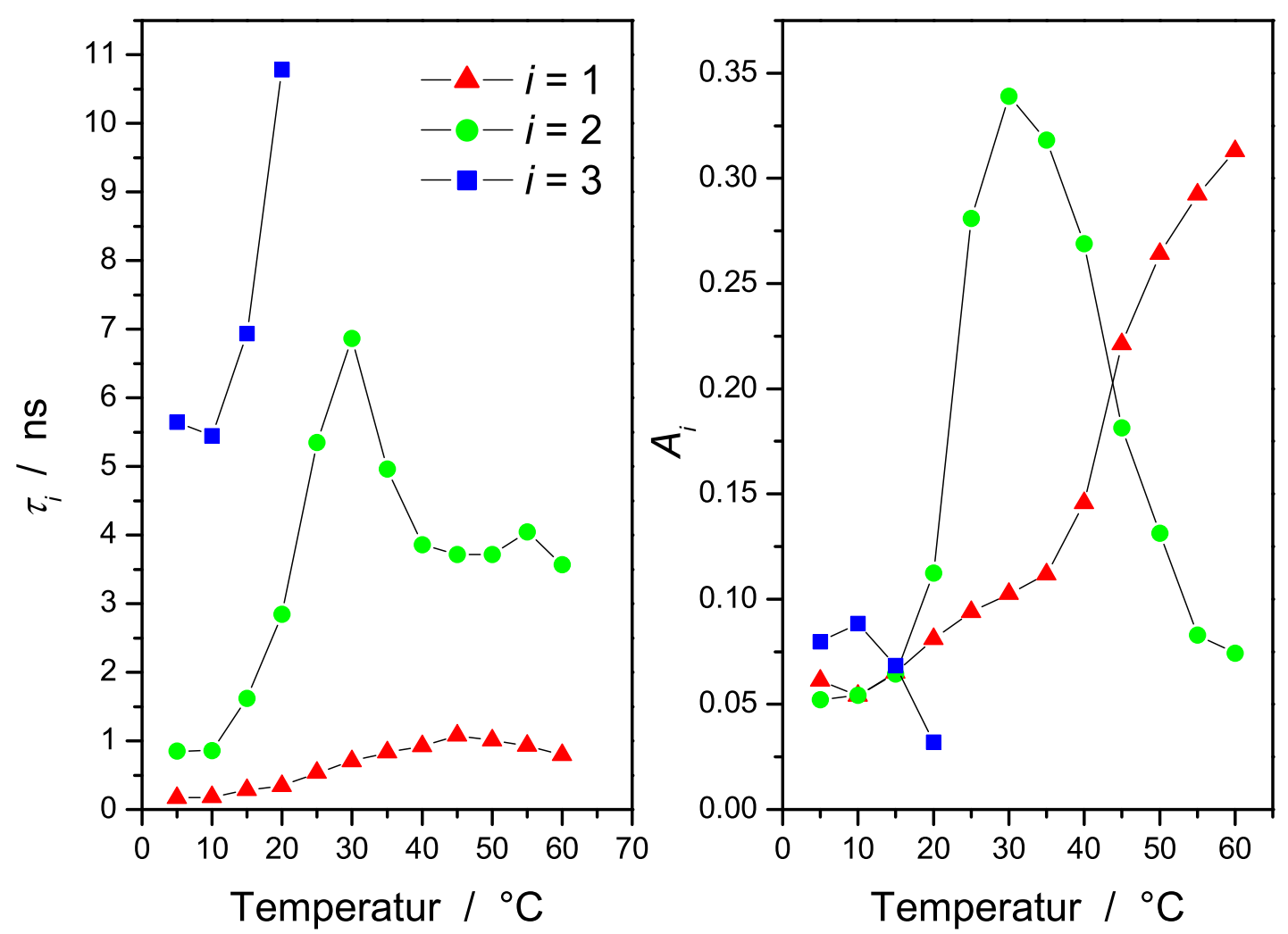

Abbildung 5.18: Graphische Darstellung der Anpassungsparameter der mit dem TCSPC-Experiment erhaltenen Solvatationskorrelationsfunktion nach Gleichung 5.28.

Um ein vereinfachtes Bild der Solvatation zu erhalten wird oftmals die mittlere Solvatationszeit $\langle\tau\rangle$ in Abhängigkeit der Temperatur angegeben, die sich durch Integration der Solvatationskorrelationsfunktion gemäß:

$$
\langle\tau\rangle=\int_{0}^{\infty} S(t) \mathrm{d} t
$$

ergibt. Die erhaltenen Werte sind in Tabelle 5.8 aufgeführt, während Abbildung 5.19 die mittlere Solvatationszeit graphisch darstellt. 


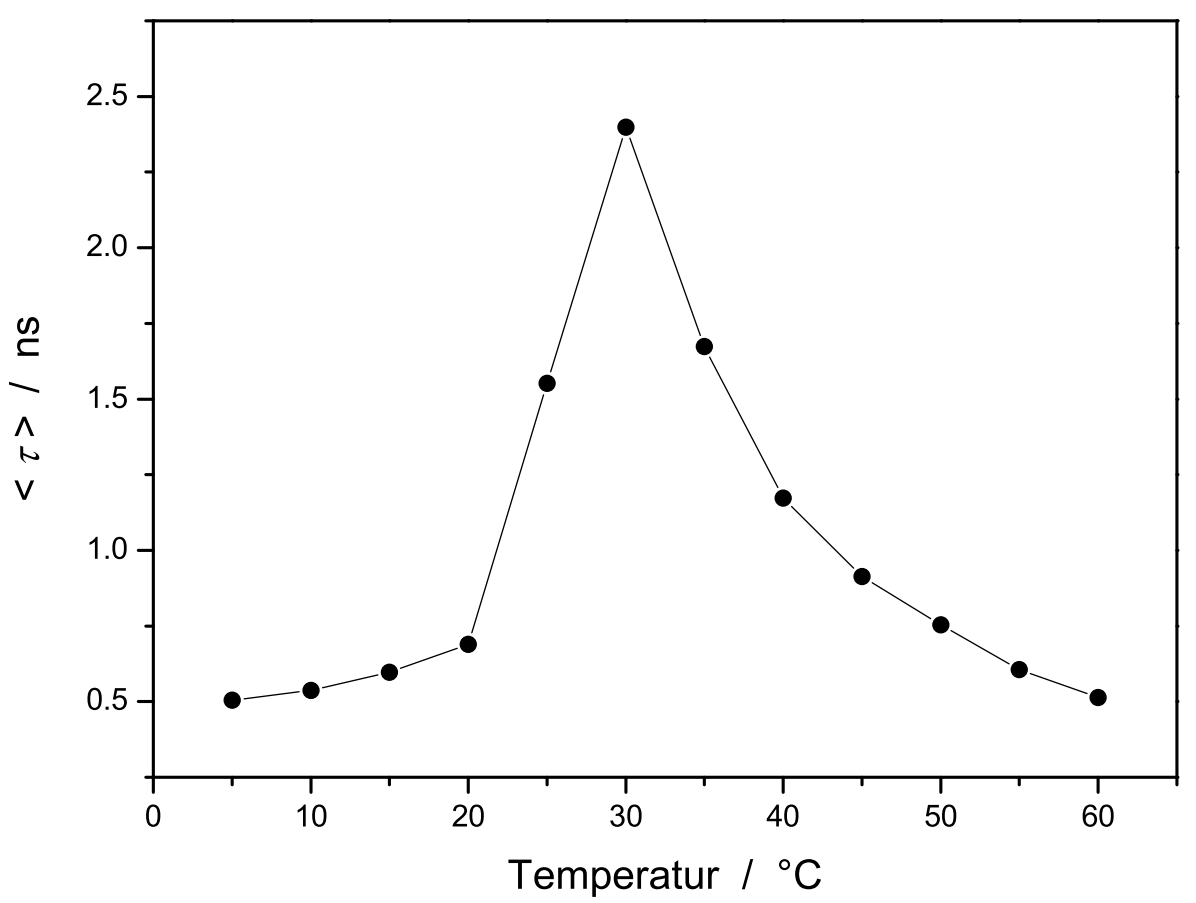

Abbildung 5.19: Mittlere Solvatationszeit $\langle\tau\rangle$ in Abhängigkeit der Temperatur.

Anhand dieser Funktion wird verdeutlicht, daß die Solvatation unterhalb des Hauptphasenübergangs im Mittel gesehen relativ schnell ist, auch wenn wie zuvor bei der Analyse der Einzelzeiten gezeigt, sehr langsam abklingende Zeitkonstanten an der Solvatation beteiligt sind. Beim Überschreiten des Hauptphasenübergangs wird die Solvatation zunächst stark verlangsamt, um mit steigender Temperatur allmählich wieder schneller zu werden. Bei dieser Betrachtungsweise handelt es sich allerdings um eine starke Vereinfachung der bei der Solvatation ablaufenden Vorgänge, die keine Aussagen bezüglich ultraschneller Zeitkomponenten zuläßt.

Einen weiteren interessanten Aspekt der Solvatation verdeutlicht Abbildung 5.20. Hier ist in Abhängigkeit von der Temperatur der theoretisch beobachtbare StokesShift $\langle\tilde{\nu}\rangle(t=0)-\langle\tilde{\nu}\rangle(t=\infty)$ dem im Experiment aufgelösten Anteil gegenübergestellt. Dieser Anteil $S(t=0)$ ergibt sich aus der Extrapolation der multiexponentiellen Anpassung der experimentell bestimmten Solvatationskorrelationsfunktion nach $t=0$ (siehe Gleichung 5.28). Die zugehörigen Werte sind in Tabelle 5.7 bzw. 5.8 in der jeweils letzten Spalte zu finden. Zunächst zeigt sich, daß mit der Technik des zeitkorrelierten Einzelphotonenzählens nur ein geringer Teil der Solvatationsdynamik erfaßt wird. Während oberhalb des Phasenübergangs noch über $40 \%$ der gesamten Solvatation aufgelöst werden kann, sind es unterhalb des Phasenübergangs lediglich $20 \%$. Offensichtlich spielen unterhalb des Hauptphasenübergangs ultraschnelle Dynamiken, die mit der Technik des 
zeitkorrelierten Einzelphotonenzählens nicht aufgelöst werden können, eine deutlich größere Rolle. Daher fällt auch die mittlere Solvatationszeit $\langle\tau\rangle$ unterhalb des Hauptphasenübergangs deutlich schneller aus. Auffallend ist weiterhin die starke Korrelation der beiden Kurvenverläufe. Da langsame Zeitkomponenten notwendig sind, um bei gegebener Zeitauflösung einen möglichst großen Anteil der Solvatationskorrelationsfunktion zu erfassen, bedeutet die beobachte Korrelation, daß gerade oberhalb des Hauptphasenübergangs die langsamen Komponenten für einen erheblichen Anteil der gesamten Solvatation verantwortlich sind. Diese Anteile lassen sich, wie im folgenden noch gezeigt werden wird, durch die größere Bewegungsfreiheit des Chromophors in der flüssig-kristallinen Phase erklären.

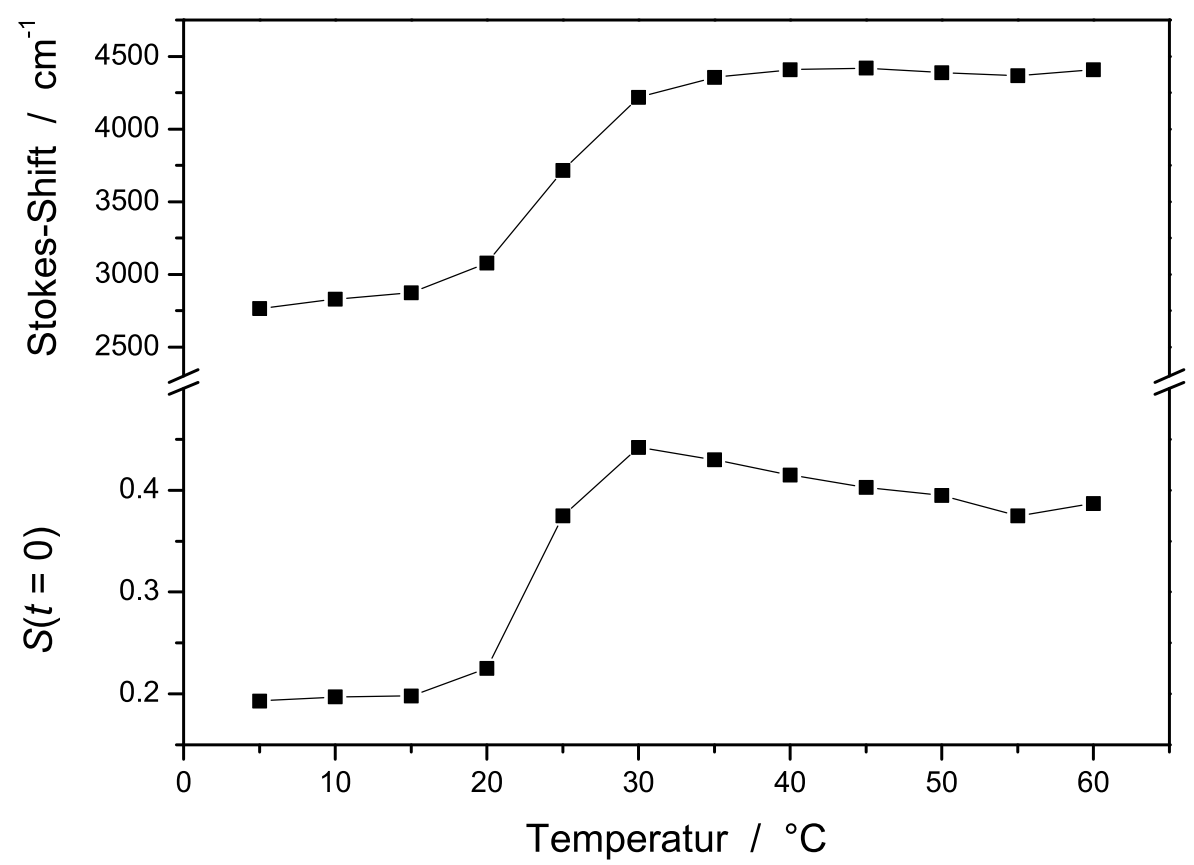

Abbildung 5.20: Gegenüberstellung des maximal auflösbaren Stokes-Shifts und des experimentell beobachteten Anteils im TCSPC-Experiment.

Dieses Unterkapitel hat gezeigt, daß bei der Solvatation in Lipidmembranen langsame Zeitkonstanten eine Rolle spielen, die in isotropen Lösungsmittelumgebungen nicht beobachtet werden. Ein erheblicher Teil der Solvatation entgeht jedoch der Beobachtung durch das zeitkorrelierte Einzelphotonenzählexperiment, weswegen Studien mit einer erheblich verbesserten Zeitauflösung durchgeführt werden müssen, um die Solvatation in Lipidmembran-Vesikeln hinreichend zu beschreiben. 


\subsubsection{Ultraschnelle Solvatation}

In Ergänzung zu den aus dem TCSPC-Experiment erhaltenen Ergebnissen wurden aufgrund des großen Anteils an nicht auflösbarer Dynamik Untersuchungen am FUC-Experiment mit einer deutlich erhöhten Zeitauflösung von ca. 0.5 ps durchgeführt (siehe Kapitel 4.4). Aufgrund des deutlich geringeren Stokes-Shifts in der gelförmigen und der Rippled-Phase konnten im Fluoreszenzkonversionsexperiment lediglich Untersuchungen oberhalb des Hauptphasenübergangs durchgeführt werden.

Wie bereits in Kapitel 4.4.4 ausführlich beschrieben, wurde die zeitabhängige Fluoreszenz für Temperaturen von $T=25$ bis $60^{\circ} \mathrm{C}$ in $5{ }^{\circ} \mathrm{C}$ Schritten aufgenommen. Für jede der Temperaturen wurden 17 Kinetiken im Bereich von 268 bis $325 \mathrm{~nm}$ (entsprechend Fluoreszenzwellenlängen von 410 bis $560 \mathrm{~nm}$ im Abstand von $10 \mathrm{~nm}$ ) über einen Zeitbereich von -100 bis 1000 ps aufgenommen.

Die einzelnen Kinetiken wurden mit einer Funktion angepaßt, die einer Faltung aus einer Gerätefunktion $G\left(t^{\prime}\right)$ und einer Summe aus Exponentialfunktionen mit zusätzlichem konstantem Offset $F\left(t^{\prime}\right)$ entspricht. Die Gerätefunktion wurde dabei als gaußförmig angenommen. Die Funktion muß weiterhin mit einer HeavysideStufenfunktion $\Phi\left(t^{\prime}\right)$ multipliziert werden, um dem Zeitpunkt der Anregung Rechnung zu tragen:

$$
\begin{aligned}
F^{*}(t) & =\int_{-\infty}^{\infty} F\left(t^{\prime}-x_{0}\right) \cdot \Phi\left(t^{\prime}-x_{0}\right) \cdot G\left(t-t^{\prime}\right) \mathrm{d} t^{\prime}, \\
& =\int_{-\infty}^{\infty}\left(\sum_{i=1}^{4} A_{i} \exp \left(-\frac{t^{\prime}-x_{0}}{\tau_{i}}\right)+A_{5}\right) \cdot \Phi\left(t^{\prime}-x_{0}\right) \cdot \exp \left(-\frac{\left(t-t^{\prime}\right)^{2}}{\sigma^{2}}\right) \mathrm{d} t^{\prime} .
\end{aligned}
$$

Die Größe $x_{0}$ in dieser Gleichung beschreibt eine Verschiebung des Signals entlang der Zeitachse, mit der der Nullpunkt des simulierten Signals angepaßt wurde [243]. Das Integral läßt sich analytisch lösen:

$$
\begin{aligned}
F^{*}(t)= & \sum_{i=1}^{4} \frac{A_{i} \sqrt{\pi} \sigma}{2} \exp \left(\left[\frac{\sigma}{2 \tau_{i}}\right]^{2}-\frac{t-x_{0}}{\tau_{i}}\right)\left(1-\operatorname{erf}\left[\frac{\sigma}{2 \tau_{i}}-\frac{t-x_{0}}{\sigma}\right]\right) \\
& +A_{5}\left(1-\operatorname{erf}\left[-\frac{t-x_{0}}{\sigma}\right]\right) .
\end{aligned}
$$

Dabei gilt:

$$
\operatorname{erf}(z)=\frac{2}{\sqrt{\pi}} \int_{0}^{z} \exp \left(-t^{2}\right) \mathrm{d} t
$$

Bei der Anpassung mit Gleichung 5.31 über ein Least-Squares-Verfahren wurden sowohl negative als auch positive Amplituden zugelassen, um ansteigende bzw. abfallende Dynamiken entsprechend wiederzugeben. Dabei wurden bis zu 
vier Exponentialfunktionen herangezogen. Bei der in der Gleichung auftretenden Zeitauflösung $\sigma$ wurde darauf geachtet, daß sie lediglich in sinnvollen Grenzen variiert wurde. Die weitere Anpassung diente lediglich der Glättung der Daten und der Bestimmung des Zeitnullpunktes. Den resultierenden Anpassungsparametern $A_{i}$ und $\tau_{i}$ wurde keine weitere Bedeutung eingeräumt.

In Abbildung 5.21 sind drei typische im FUC-Experiment erhaltene Meßkurven bei 420, 480 und $540 \mathrm{~nm}$ für Laurdan in DMPC-Vesikeln bei $T=35^{\circ} \mathrm{C}$ mit der jeweiligen Anpassungsfunktion dargestellt. Sämtliche Kinetiken wurden anhand der aus dem TCSPC-Experiment erhaltenen, zeitaufgelösten Fluoreszenzspektren bei $t=0.531 \mathrm{~ns}$ bei entsprechender Temperatur gewichtet.

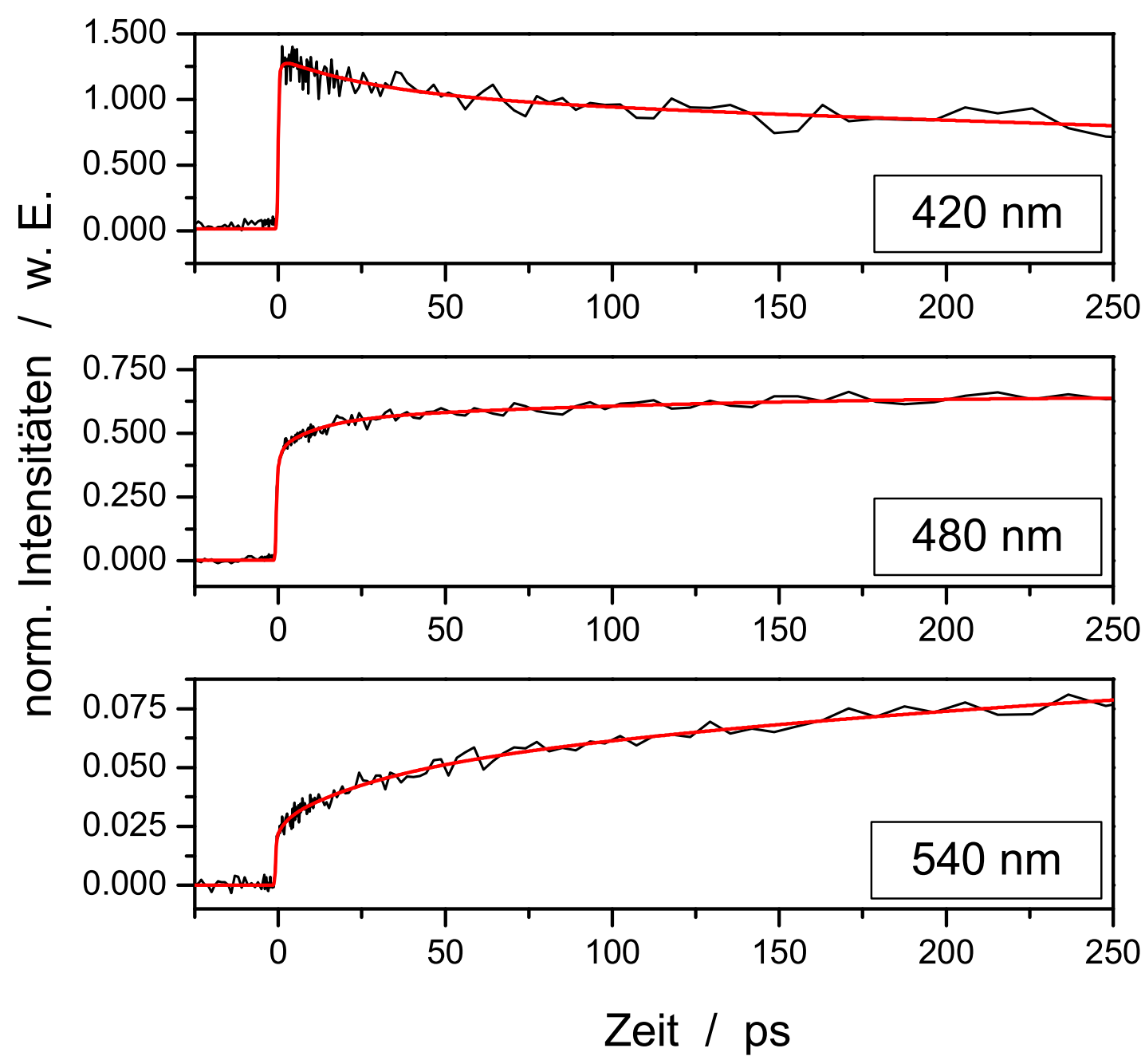

Abbildung 5.21: Typische Kinetiken für Laurdan in $D M P C$ bei $T=35{ }^{\circ} \mathrm{C}$ mit Anpassungen nach Gleichung 5.31. 
Aus den derart gewonnenen Kinetiken wurden schließlich die zeitaufgelösten Fluoreszenzspektren rekonstruiert. Eine Anpassung der Spektren mit zwei Gaußfunktionen wie im TCSPC-Experiment war hier aufgrund der stark verminderten Punktanzahl pro Spektrum nicht befriedigend. Daher wurde der Anpassung mit einer zeitabhängigen Log-Normal-Funktion nach Gleichung 5.9 mit einer verminderten Anzahl an Anpassungsparametern der Vorzug gegeben. Die Auswertung der Spektren erfolgte analog dem TCSPC-Experiment über die Berechnung der statistischen Momente. Die zweiten und dritten zentralen Momente, d. h. Breite und Asymmetrie, liefern dabei keine wesentlich neuen Aspekte für die Untersuchung der Solvatationsdynamik. Von Interesse ist jedoch insbesondere die Erweiterung der Solvatationskorrelationsfunktion zu kurzen Zeiten. Die Bestimmung der Schwerpunkte über das erste Moment führte dabei nach Gleichung $5.20 \mathrm{zu}$ einer nach kurzen Zeiten erweiterten Solvatationskorrelationsfunktion. Um einen fließenden Übergang zwischen den aus TCSPC- und aus FUC-Daten gewonnenen Korrelationsfunktionen zu gewährleisten, wurde eine moderate Verschiebung der aus den FUC-Daten erhaltenen Korrelationsfunktion bezüglich der Ordinate $S$ zugelassen. Eine Auswahl der erhaltenen Solvatationskorrelationsfunktionen sind in Abbildung 5.22 in einer halblogarithmischen Auftragung von $50 \mathrm{fs}$ bis $20 \mathrm{~ns}$ dargestellt.

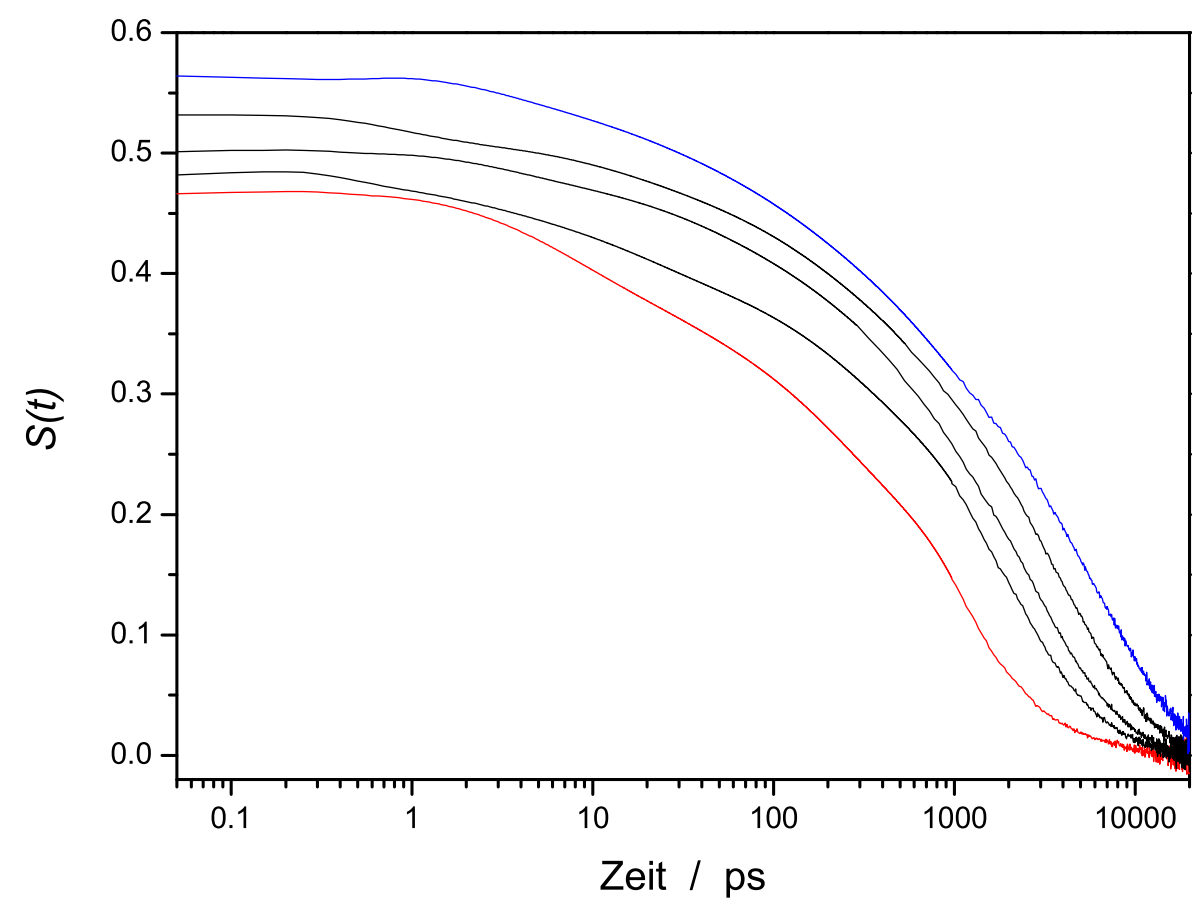

Abbildung 5.22: Halblogarithmische Darstellung der Solvatationskorrelationsfunktion von Laurdan in DMPC bei ausgewählten Temperaturen ( $T=30$ (blau), $35,40,45$ und $60^{\circ} \mathrm{C}$ (rot)). 
Die Funktionen zeigen ein stark multiexponentielles Abklingverhalten. Sie wurden bis ca. 200 ps mit einer Summe aus drei Exponentialfunktionen und variablem Offset angepaßt:

$$
S(t)=\sum_{i=1}^{3} A_{i} \exp \left(-\frac{t}{\tau_{i}}\right)+A_{4} .
$$

Die erhaltenen Anpassungsparameter sind in Tabelle 5.9 zusammengefaßt, während Abbildung 5.23 die erhaltenen Zeitkonstanten und Amplituden graphisch darstellt.

\begin{tabular}{|c|c|c|c|c|c|c|c|c|}
\hline $\boldsymbol{T} /{ }^{\circ} \mathbf{C}$ & $\boldsymbol{A}_{\mathbf{1}}$ & $\boldsymbol{\tau}_{\mathbf{1}} / \mathbf{p s}$ & $\boldsymbol{A}_{\mathbf{2}}$ & $\boldsymbol{\tau}_{\mathbf{2}} / \mathbf{p s}$ & $\boldsymbol{A}_{\mathbf{3}}$ & $\boldsymbol{\tau}_{\mathbf{3}} / \mathbf{p s}$ & $\boldsymbol{A}_{\mathbf{4}}$ & $\boldsymbol{S}(\boldsymbol{t}=\mathbf{0})$ \\
\hline 25 & 0.017 & 5.24 & 0.039 & 22.8 & 0.116 & 296.6 & 0.277 & 0.449 \\
30 & 0.027 & 3.67 & 0.034 & 22.1 & 0.138 & 210.1 & 0.372 & 0.571 \\
35 & 0.027 & 0.87 & 0.032 & 15.4 & 0.130 & 211.4 & 0.349 & 0.538 \\
40 & 0.024 & 3.48 & 0.033 & 31.7 & 0.149 & 308.0 & 0.299 & 0.506 \\
45 & 0.025 & 1.51 & 0.052 & 15.2 & 0.138 & 253.4 & 0.271 & 0.486 \\
50 & 0.065 & 0.88 & 0.038 & 11.2 & 0.174 & 132.1 & 0.256 & 0.532 \\
55 & 0.028 & 1.28 & 0.064 & 12.2 & 0.187 & 260.7 & 0.209 & 0.489 \\
60 & 0.061 & 6.24 & 0.039 & 22.8 & 0.181 & 244.0 & 0.192 & 0.473 \\
\hline
\end{tabular}

Tabelle 5.9: Anpassungsparameter der mit dem FUC-Experiment erhaltenen Solvatationskorrelationsfunktion und experimentelles Zeitnullpunktsspektrum.

Es wird für alle Temperaturen eine langsame Zeitkonstante um 250 ps beobachtet, deren Amplitude mit steigender Temperatur deutlich zunimmt. Im Gegenzug nimmt der verwendete konstante Offset von $30^{\circ} \mathrm{C}$ ausgehend stark ab. Die beiden schnelleren Zeitkonstanten weisen Amplituden auf einem konstant niedrigem Niveau auf. Die mittlere Zeitkonstante ist dabei um 20 ps zentriert, während die schnellste Zeitkonstante eine stark Streuung von ca. 0.9 bis 6 ps aufweist.

Es zeigt sich, daß mit Hilfe der Fluoreszenzkonversionsspektroskopie in der Tat Komponenten im Pikosekundenbereich gefunden werden, die im TCSPCExperiment nicht beobachtet werden konnten. Dennoch entgehen auch hier noch immer ca. $50 \%$ der Solvatationskorrelationsfunktion der Detektion. Offensichtlich spielen bei der Solvatation an der Grenzschicht von Lipidmembranen ultraschnelle Zeitkomponenten im Bereich unter ca. 500 fs eine erhebliche Rolle. Die Resultate werden im folgenden diskutiert. 

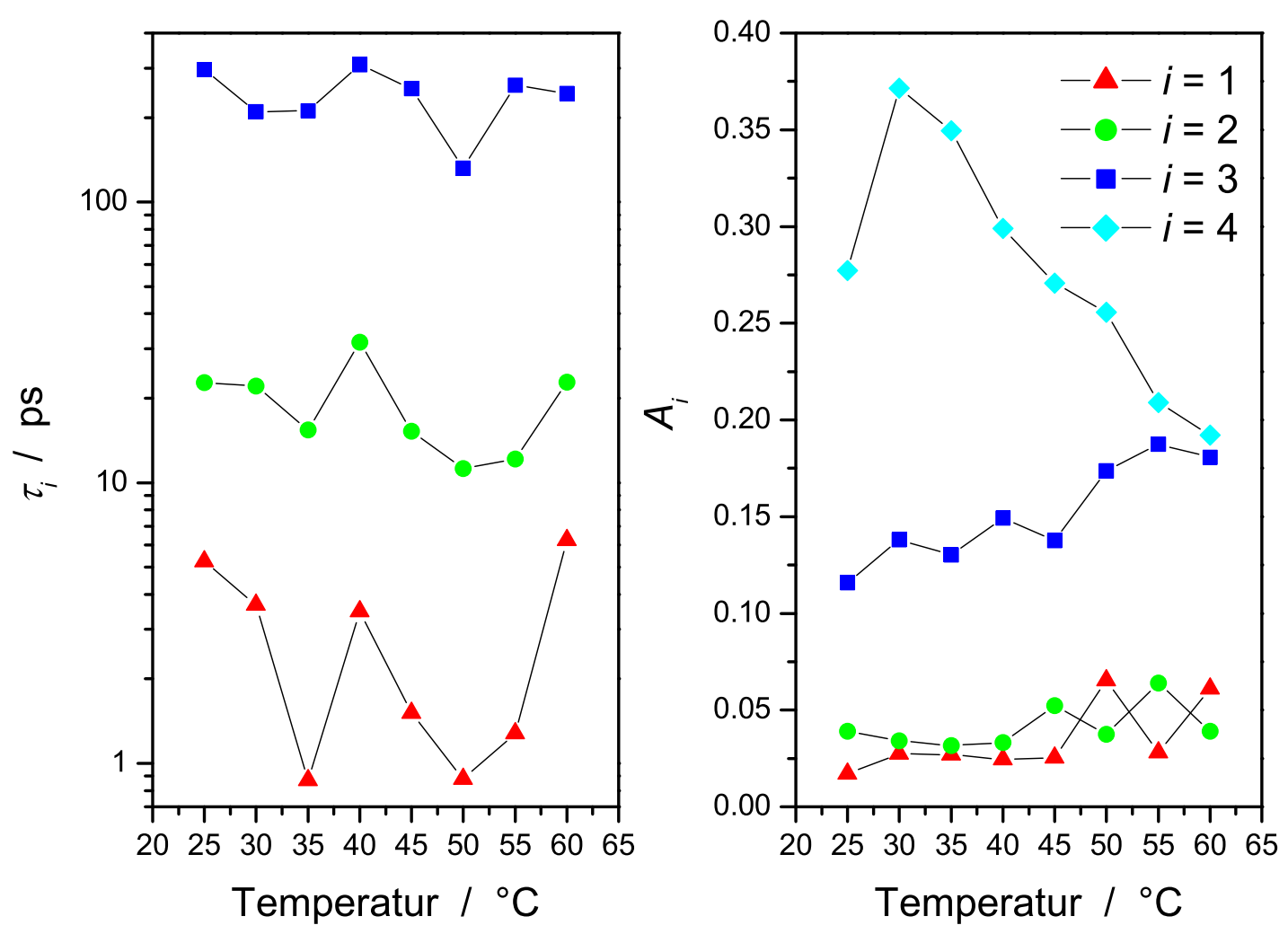

Abbildung 5.23: Graphische Darstellung der Anpassungsparameter der mit dem FUC-Experiment erhaltenen Solvatationskorrelationsfunktion.

\subsection{Diskussion der Solvatationsdynamik}

\section{Zeitkorreliertes Einzelphotonenzählen}

Die Untersuchung der Solvatationsdynamik mit Hilfe des zeitkorrelierten Photonenzählens bringt zum Ausdruck, daß langsame Komponenten im Nanosekundenbereich eine bedeutende Rolle bei der Solvatation in Lipidmembranumgebungen spielen, die in reinem Wasser nicht beobachtet werden. Diese langsamen Zeitkomponenten sind ein gemeinsames Phänomen bei der Beobachtung in heterogenen, biologischen Systemen, wie in Kapitel 3 deutlich geworden ist. Von Bhattacharyya und Mitarbeitern wurden in vergleichbaren vesikulären Systemen ähnliche Zeitkomponenten gefunden, die der Solvatation durch gebundenes Wasser im Inneren der Vesikel zugeordnet wurden [89,90]. Auch Parasassi und Mitarbeiter weisen diese langsamen Zeitkomponenten der Relaxation des in der Membran befindlichen Wassers zu [142]. Von Nandi und Bagchi wird die Ursache der langsamen Zeitkomponenten durch einen dynamischen Austausch zwischen ge- 
fangenen und gebunden Wasserspezies zu erklären versucht [98]. Andere Arbeiten weisen darauf hin, daß die Dynamik der Lipidmoleküle und/oder der verwendeten Farbstoffsonden ursächlich für das Auftauchen der langsamen Zeitkomponenten sein könnten [50,79]. Die unterschiedlichen Interpretationen machen deutlich, daß die Mechanismen der Solvatation in eingeschränkten Umgebung noch einen hohen Untersuchungsbedarf aufweisen. Wie nachfolgend gezeigt wird, lassen sich die langsamen Zeitkomponenten im hier vorgestellten DMPC/Laurdan-System vorrangig durch die diffusive Bewegung des Chromophors in seiner eingeschränkten Lipidumgebung erklären, wodurch die Solvatation durch Wasser auf diesen langsamen Zeitskalen hier als unwahrscheinlich zu erachten ist.

Wie bereits angemerkt wurde, ist die auffällige Ähnlichkeit der zeitaufgelösten Anisotropie $r(t)$ und des zeitaufgelösten Stokes-Shifts in Abhängigkeit der Temperatur nicht zufällig (vergleiche Abbildung 5.7 und 5.13). Der Zusammenhang zwischen den beiden Funktionen ist bereits durch einen Vergleich des Integrals $F$ unter der Anisotropiefunktion $r(t)$ mit der mittleren Solvatationszeit $\langle\tau\rangle$ anschaulich darstellbar (siehe Gleichung 5.6 bzw. 5.29). $F$ und $\langle\tau\rangle$ wurden durch die gleichen mathematischen Operationen an vollkommen unabhängigen Meßreihen erhalten. Die bei $5{ }^{\circ} \mathrm{C}$ auf Null gesetzten und normierten Funktionen sind in Abbildung 5.24 dargestellt.

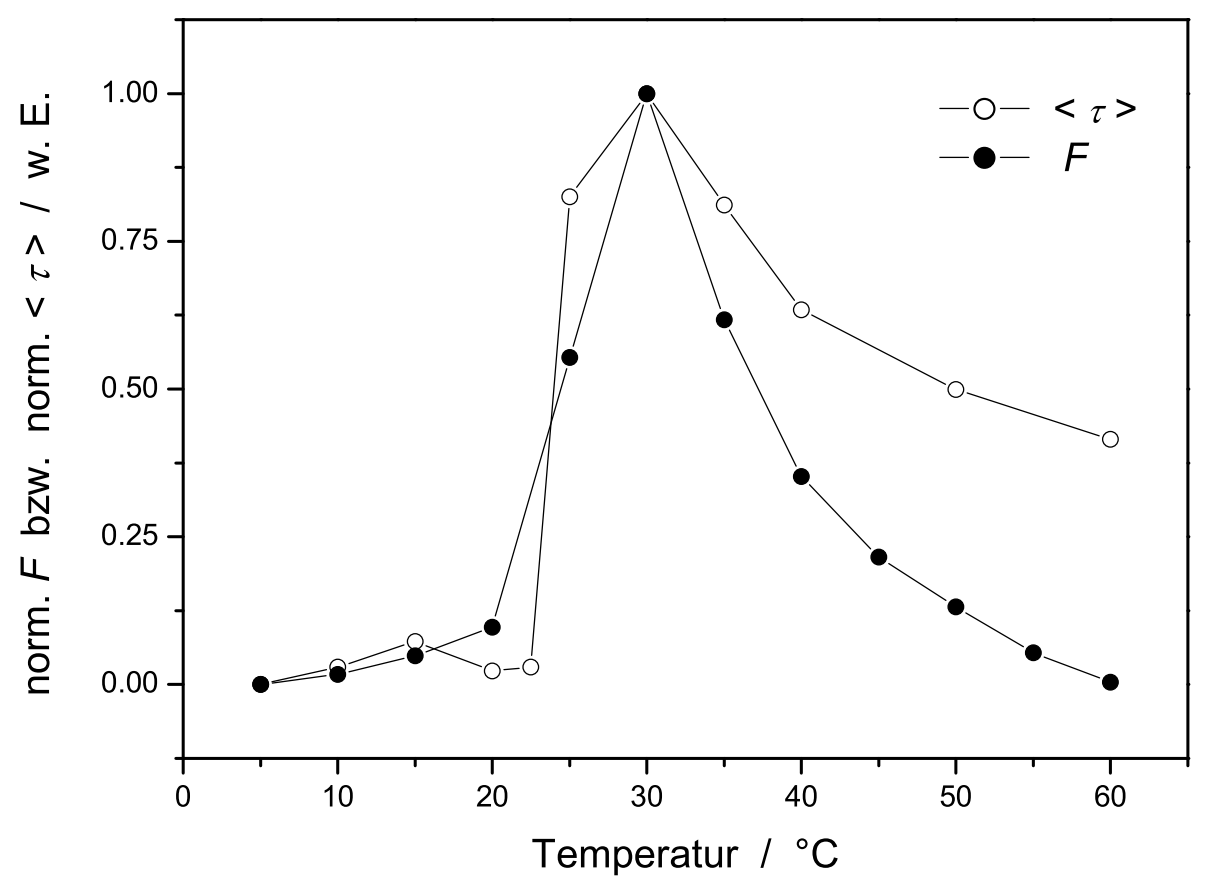

Abbildung 5.24: Vergleich zwischen der Temperaturabhängigkeit des Integrals unter der zeitabhängigen Anisotropie $F$ nach Gleichung 5.6 und der mittleren Solvatationszeit $\langle\tau\rangle$ nach Gleichung 5.29. 
Die starke Korrelation weist bereits darauf hin, daß die beiden zunächst unabhängigen Aspekte auf vergleichbare Weise durch die Temperatur beeinflußt werden. Es liegt daher nahe, die Dynamiken der beiden Funktionen im Detail zu vergleichen. Dazu sind in Abbildung 5.25 die Parameter aus der biexponentiellen Anpassung der Anisotropiefunktion $r(t)$ aus Tabelle 5.4 den Anpassungsparametern der Solvatationskorrelationsfunktion aus Tabelle 5.8 gegenübergestellt. Dabei sind die Messungen bei 20 und $22.5^{\circ} \mathrm{C}$ der Anisotropiefunktion nicht dargestellt.
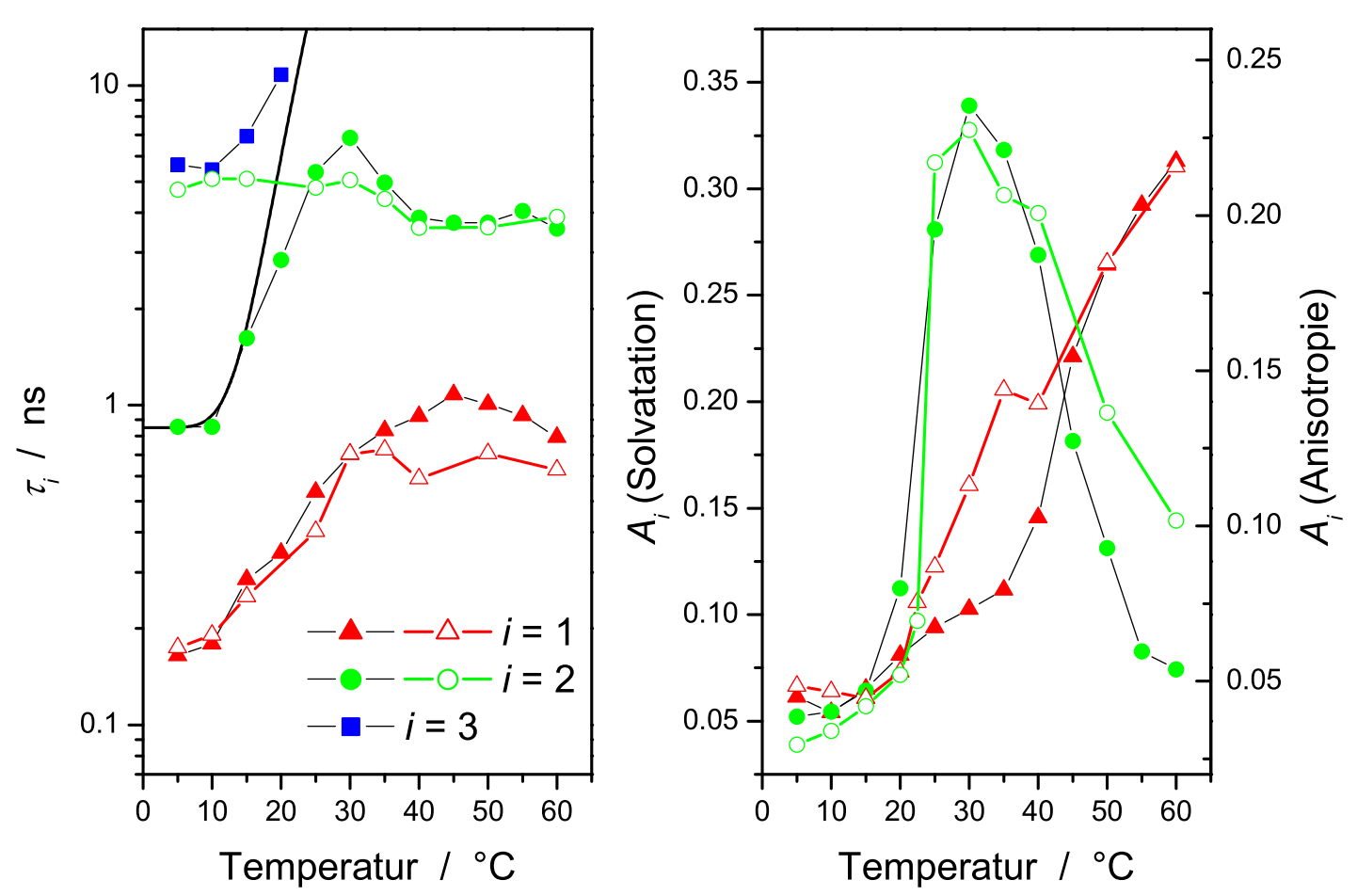

Abbildung 5.25: Gegenüberstellung der Anpassungsparameter der mit dem TCSPC-Experiment erhaltenen Anisotropiefunktion nach Gleichung 5.2 (leere Symbole) und der Solvatationskorrelationsfunktion nach Gleichung 5.28 (gefüllte Symbole).

Es zeigt sich, daß im Rahmen der Meßgenauigkeit die beiden unabhängigen Meßreihen durch vergleichbare Zeitkonstanten wiedergegeben werden. Insbesondere der charakteristische Verlauf der Amplituden ist dabei ein deutlicher Hinweis auf die gemeinsamen Ursachen der beobachteten Dynamiken. Unterhalb des Hauptphasenübergangs sind die Verhältnisse verkompliziert, da hier offensichtlich eine dritte Zeitkomponente eine Rolle spielt, welche den beiden anderen Dynamiken überlagert ist. Der ungefähre Verlauf dieser dritten Zeitkomponente ist in der Abbildung durch eine schwarze Kurve skizziert. Die Herkunft dieser drit- 
ten Komponente ist ungeklärt, da für weitere Relaxationsmechanismen innerhalb einer Membranumgebung, wie beispielsweise der Lipidkettenbewegung, eher ein beschleunigender Effekt mit ansteigender Temperatur erwartet würde. Die Amplitude dieses Freiheitsgrades ist zudem sehr gering (siehe Abbildung 5.18). Aufgrund der unzureichenden Informationen wird von einer weiteren Diskussion dieser Komponente abgesehen.

Die Komponente unterhalb einer Nanosekunde (rot) und die zwischen 4 und 6 ns befindliche Komponente (blau unterhalb bzw. grün oberhalb des Hauptphasenübergangs) der Solvatationskorrelationsfunktion ist aufgrund der auffälligen Übereinstimmung mit den Anpassungsparametern der Anisotropiefunktion offensichtlich auf die Bewegungsfreiheit des Chromophors innerhalb seiner Membranumgebung zurückzuführen. Die Mechanismen der Rotationsdiffusion um die lange Achse des Chromophors und der Wobbling-Diffusion des Chromophors in einem eingeschränkten Raumwinkel spielen offenbar bei der Solvation eines erzeugten Nichtgleichgewichtszustandes auf einer Nanosekunden-Zeitskala eine entscheidende Rolle. Auch die Temperaturabhängigkeit der Solvatationskorrelationsfunktion im TCSPC-Experiment ist damit hinreichend erklärt. Durch das Schmelzen der Kettenregion der Membran mit steigender Temperatur, wird dem Chromophor eine erhöhte Bewegungsfreiheit eingeräumt, wodurch ihm die Möglichkeit gegeben ist, bei der Stabilisierung eines erzeugten Nichtgleichgewichtszustandes stärker teilzuhaben. Die Solvatation durch Wasser ist daher nicht notwendig, um das charakteristische Temperaturverhalten der Solvatationskorrelationsfunktion in diesem Zeitbereich zu erklären. In dem hier untersuchten System scheint die Dynamik der Solvatation im Gegensatz zu den Beobachtungen von Bhattacharyya bzw. Parasassi und Mitarbeitern auf einer Nanosekunden-Zeitskala vorrangig durch die Eigendynamik des Chromophors in seiner eingeschränkten Umgebung bestimmt zu sein.

\section{Fluoreszenzaufkonvertierung}

Im Fluoreszenzkonversionsexperiment werden drei Zeitkomponenten gefunden. Die langsamste der Zeitkomponenten wurde mit variablen Offset angepaßt und liegt bei einigen hundert Pikosekunden. Diese Komponente könnte allerdings auch mit der schnellsten Zeitkomponente aus dem TCSPC-Experiment wiedergegeben werden. Da hier aber vorrangig die schnelleren Zeitkonstanten untersucht werden sollen, wurde einer von den TCSPC-Daten unabhängigen Auswertung der Fluoreszenzkonversionsdaten der Vorzug gegeben, was zu deutlich besseren Anpassungen bei kurzen Zeiten führte. Damit ergeben sich für diese Komponente zwar etwas schnellere Zeitkonstanten als in den TCSPC- bzw. Anisotropie-Experimenten, die Herkunft der langsamen Zeitkonstante im FUC-Experiment liegt aber vermut- 
lich dennoch in der Eigendynamik des Chromophors. Dafür spricht auch das bereits im TCSPC-Experiment beobachtete Ansteigen der zugehörigen Amplitude mit steigender Temperatur.

Interessant in diesem Zusammenhang ist, daß wie in Kapitel 3.4.4 dargestellt, von Vöhringer und Mitarbeitern in 3-Puls Photon-Echo Peakshift Studien sowohl in reinem Wasser als auch an Lipidmembran/Wasser-Grenzschichten eine Zeitkonstante vergleichbarer Größe im Bereich einiger hundert Pikosekunden gefunden wurde $[50,79]$. Auffallend ist aber, daß sich diese Zeitkomponente beim Einbringen des Chromophors C18-DiD in die Grenzschicht über eine nicht-kovalente Verankerung nicht signifikant ändert. Ebenso erweist sich diese Komponente bei Variation der Temperatur im Rahmen der Meßgenauigkeit als konstant. Die Ursache dieser Beobachtung könnte damit begründet werden, daß der in diesen Arbeiten verwendete Chromophor C18-DiD über zwei aliphatische Kohlenstoffketten in der Membran verankert ist, wodurch die Rotationsdiffusion dieser Farbstoffsonde signifikant verlangsamt bzw. vollkommen unterdrückt sein sollte. Der Ursprung der langsamen Zeitkonstanten ist daher in diesem System mit hoher Wahrscheinlichkeit nicht auf die Eigendynamik des Chromophors zurückzuführen. Nach Vöhringer und Mitarbeitern könnte es sich um die kollektive Rotationsdiffusion von Wassermolekülen handeln [50,79]. Dafür spricht auch, daß die gleiche Zeitkomponente in reinem Wasser gefunden wird. Eine vergleichbare Zeitkomponente wurde von Levinger und Mitarbeitern ebenfalls in reinem Wasser und im Wassereinschluß inverser Mizellen gefunden (siehe Kapitel 3.3.2) [105]. Auch in deren Arbeit wurde diese Komponente kollektiven Bewegungen des Wassers zugeschrieben. Daher ist es nicht ausgeschlossen, daß auch in dem hier untersuchten Laurdan/DMPCSystem die kollektive Rotationsdiffusion des Wassers eine Rolle spielt und den Prozessen der Chromophordynamik auf einer Zeitskala von einigen hundert Pikosekunden überlagert ist. Die Herkunft der beiden ultraschnellen Zeitkomponenten soll im folgenden diskutiert werden.

Nach Viard et al. findet in unpolaren Lösungsmitteln auf einer Zeitskala von einigen zehn Pikosekunden eine Umwandlung des lokal angeregten Zustandes von Laurdan in einen Charge-Transfer-Zustand (CT) statt [171]. Es sprechen einige Beobachtungen dafür, daß auch in einer Lipidmembranumgebung CT-Zustände auftreten und daher eine solche Reaktion in dem hier untersuchten System eine Rolle spielen könnte. So haben die Untersuchungen der Fluoreszenzlebensdauer in Kapitel 5.4.1 gezeigt, daß sich oberhalb des Hauptphasenübergangs ein zusätzlicher, temperaturabhängiger Desaktivierungskanal öffnet, der der Bildung eines CT-Komplexes zuzuschreiben sein könnte. Weiterhin spricht dafür, daß sich die zeitabhängigen Fluoreszenzspektren oberhalb des Hauptphasenübergangs mit einer Summe aus zwei Gaußfunktionen beschreiben lassen, wovon eine temperaturabhängig auf einer Zeitskala von einigen hundert Pikosekunden bis wenigen Nanosekunden abklingt. In der flüssig-kristallinen Phase ist das Auftauchen eines CTKomplexes demnach wahrscheinlich. Die im Fluoreszenzkonversionsexperiment 
(FUC) beobachtete Zeitkonstante $\tau_{2}$ um 20 ps könnte daher der Umwandlung des lokal angeregten Zustandes in einen Charge-Transfer-Komplex zuzuschreiben sein. Leider war es nicht möglich, die FUC-Experimente auch unterhalb des Hauptphasenübergangs durchzuführen, was die hier vorgestellte Interpretation durch die Abwesenheit der mittleren Zeitkomponente $\tau_{2}$ unterhalb des Hauptphasenübergangs unterstützen würde. Da eine Zuordnung von $\tau_{2}$ zu einer strukturellen Umwandlung des Chromophors aufgrund der unzureichenden Informationen in der vorliegenden Arbeit nicht verifiziert werden kann, soll nach alternativen Interpretationsmöglichkeiten gesucht werden. Darüberhinaus bliebe die Herkunft der schnellsten im FUC-Experiment beobachteten Zeitkomponente $\tau_{1}$ weiterhin ungeklärt.

Da von Vöhringer und Mitarbeitern gezeigt werden konnte, daß die Solvatation auch in einer Membranumgebung noch vorrangig durch die ultraschnelle Dynamik des Wassers bestimmt ist, liegt es nahe in der Membran befindliches Wasser für das Auftauchen der ultraschnellen Zeitkomponenten verantwortlich zu machen $[27,50,79]$. Allerdings ist der in diesen Arbeiten verwendete Chromophor C18-DiD weniger tief in der Membran als das hier verwendete Laurdan verankert, wodurch er eine deutlich wasserähnlichere Umgebung besitzt. Daher ist ein direkter Vergleich mit den hier vorgestellten Untersuchungen nur bedingt möglich.

Wie bereits in Kapitel 3.1 gezeigt, ist die Solvatationsdynamik des reinen Wassers auf ultraschnellen Zeitskalen durch zwei charakteristische Zeitkomponenten bestimmt. Dabei ist die langsamere der beiden Zeitkonstanten um 10 ps auf die Rotationsdiffusion individueller Wassermoleküle in der freien Wasserphase zurückzuführen, während die schnellere der Komponenten um 1 ps den gehinderten Translationsmoden des reinen Wassers zuzuordnen ist. Diese mit unterschiedlichen Meßtechniken ermittelten Komponenten wurden von Vöhringer und Mitarbeitern im Vergleich zu eigenen Messungen des optischen Kerr-Effektes (OKE) diskutiert [45]. Abbildung 5.26 gibt eine Auswahl der in dieser Arbeit behandelten Relaxationszeiten in Abhängigkeit der Temperatur wieder. Dabei ist zu bemerken, daß für einige dieser Techniken die erhaltenen Relaxationszeiten nach einem von Lynden-Bell et al. abgeleiteten Zusammenhang mit drei multipliziert werden müssen, damit die mit unterschiedlichen Meßtechniken erhaltenen Relaxationszeiten verglichen werden können $[45,244,245,246]$. Im folgenden sollen die im Fluoreszenzkonversionsexperiment erhaltenen Zeitkonstanten anhand dieser Arbeiten interpretiert werden.

Die mittlere der im FUC-Experiment bestimmten Zeitkomponenten besitzt eine sehr geringe Amplitude und bewegt sich im Bereich von $\tau_{2}=20$ ps. Ihr Fehler beträgt dabei ca. \pm 3 ps. Die Zeitkonstante nimmt mit steigender Temperatur tendenziell ab. Die schnellste Zeitkonstante $\tau_{1}$ weist eine starke Streuung im Bereich weniger Pikosekunden bei sehr geringer Amplitude auf. Dabei ist zu beachten, daß diese Komponente aufgrund der in der vorliegenden Arbeit auf ca. 0.5 ps be- 


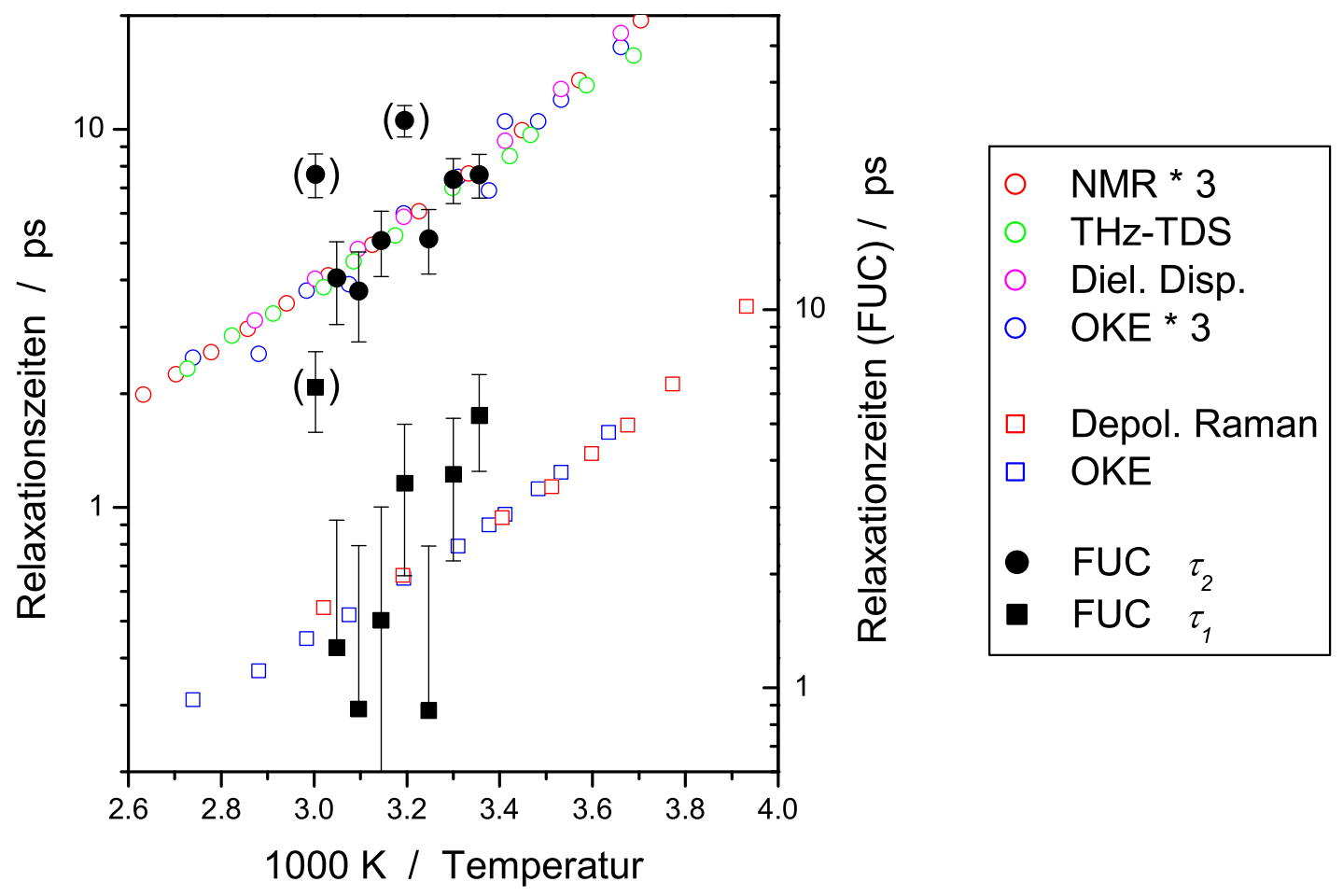

Abbildung 5.26: Vergleich der charakteristischen Relaxationszeiten für reines Wasser nach Winkler et al. [45] mit den in FUC-Experimenten ermittelten Zeitkonstanten (Anm.: Für die gefüllten Symbole ist die rechte Ordinate maßgeblich).

schränkten Zeitauflösung nur sehr grob bestimmt werden kann und ihr Fehler zu $\pm 1.5 \mathrm{ps}$ angenommen wird. Tendenziell nimmt auch $\tau_{1}$ mit steigender Temperatur ab. Die Solvatationszeiten sind in Abbildung 5.26 den Relaxationszeiten des reinen Wassers in einer halblogarithmischen Auftragung gegen die reziproke, absolute Temperatur gegenübergestellt. Dabei ist zu beachten, daß für die aus dem Fluoreszenzkonversionsexperiment ermittelten Zeitkonstanten eine andere Ordinate als bei den Relaxationszeiten des reinen Wassers gewählt wurde, die sich um einen Faktor 3 von der des reinen Wassers unterscheidet. Es zeigt sich, daß bei dieser Auftragung mit Ausnahme der Messungen für $T=40$ und $60^{\circ} \mathrm{C}$ eine gute Übereinstimmung der mittleren Zeitkomponenten $\tau_{2}$ mit der Zeitkonstanten für diffusive Rotationsmoden einzelner Wassermoleküle erzielt wird. Auch die Temperaturabhängigkeit wird dabei wiedergegeben. Für die schnelle Zeitkonstante $\tau_{1}$ ergibt sich mit Ausnahme der Messung für $T=60^{\circ} \mathrm{C}$ eine grobe Übereinstimmung mit der Zeitkomponenten der gehinderten Translationsmoden des reinen Wassers. Die Konstanten weisen allerdings wie bereits angemerkt eine starke Streuung auf, so daß die Temperaturabhängigkeit hier nicht eindeutig wiedergegeben wird. Bemerkenswert ist dabei aber, daß das Verhältnis der schnelleren zur langsameren Zeitkonstanten in der Membran das gleiche wie in der reinen Wasserphase ist. 
Unter Berücksichtigung der Tatsache, daß der in der vorliegenden Arbeit verwendete Chromophor vergleichsweise tief innerhalb der Kopfgruppenregion der Membran verankert ist und daher eine deutlich unterschiedliche Wasserumgebung im Vergleich zur reinen Wasserphase erfährt, ist es plausibel anzunehmen, daß räumliche Einschränkungen in der Kopfgruppenregion zu einer deutlichen Verlangsamung der Relaxationsbewegungen von in dieser Region gebundenem Wasser führen. Es liegt daher nahe, die mittlere Zeitkomponente $\tau_{2}$ den diffusiven Rotationen individueller Wassermoleküle innerhalb der Kopfgruppenregion der Lipidmembranen zuzuordnen. Dafür spricht auch die wiedergegebene Temperaturabhängigkeit dieser Komponenten. Unter Berücksichtigung, daß die schnelle Zeitkonstante $\tau_{1}$ aufgrund der beschränkten Zeitauflösung und der geringen Amplitude nur sehr schwer zu bestimmen ist, wird diese Komponente mit den Translationsmoden des Wassers in der Kopfgruppenregion assoziiert. Für die hier getroffene Zuordnung der beobachteten Zeiten zu den Freiheitsgraden des Wasser spricht vor allem, daß das Verhältnis zwischen der schnellen und der mittleren Zeitkonstanten in der Lipidmembran dem Verhältnis der beiden charakteristischen Zeitkonstanten des reinen Wassers entspricht. Offensichtlich sind die Wassermoleküle in einer solch eingeschränkten Umgebung jedoch deutlich in ihrer Bewegungsfreiheit gehindert, weswegen sie eine verlangsamte Dynamik um etwa den Faktor 3 aufweisen.

Die Ausführungen zeigen, daß trotz der tiefen Einbaulage des Chromophors, Wasser zumindest in der flüssig-kristallinen Phase weiterhin eine Rolle bei der Solvatation eines erzeugten Nichtgleichgewichtszustandes spielt. Entgegen den Beobachtungen von Parasassi, Bhattacharyya und Nandi findet die Solvatation durch Wasser innerhalb einer Membranumgebung allerdings noch immer auf einer Pikosekunden-Zeitskala statt, auch wenn die charakteristischen Zeitkonstanten des Wassers durch die Einschränkung der Umgebung um einen Faktor 3 verlangsamt erscheinen. Aufgrund der geringen Konzentration des Wassers in der Membran, besitzen diese Freiheitsgrade allerdings eine sehr geringe Amplitude, was ihre Bestimmung deutlich erschwert. Da die Fluoreszenzkonversionsexperimente lediglich in der flüssig-kristallinen Phase durchgeführt wurden, läßt sich nicht entscheiden, ob Wasser auch unterhalb des Hauptphasenübergangs zur Solvatation beiträgt. Der nicht auflösbare Anteil der Solvatationskorrelationsfunktion von ca. $80 \%$ im TCSPC-Experiment deutet allerdings darauf hin.

Es ist weiterhin anzumerken, daß trotz der Zeitauflösung von ca. 0.5 ps auch in der hier untersuchten flüssig-kristallinen Phase lediglich etwas mehr als die Hälfte des gesamten zeitabhängigen Stokes-Shifts erfaßt werden konnte. Der übrige Teil entzieht sich der Beobachtung mit den hier durchgeführten Techniken. Es kann jedoch vermutet werden, daß dieser Anteil auch in Membranumgebungen einem ultraschnellen Inertialteil zuzuordnen ist, welcher sich auf Zeitskalen unter hundert Femtosekunden abspielt [50]. 


\subsection{Resümee - Lipid-Vesikel}

In diesem Kapitel wurde anhand zeitaufgelöster Fluoreszenzmessungen mit Hilfe der Technik des zeitkorrelierten Einzelphotonenzählens und der Fluoreszenzkonversionsspektroskopie die Solvatation eines nicht-kovalent in einer Lipidmembran verankerten und in der Region der Kopfgruppen befindlichen Chromophors untersucht. Der Vergleich zwischen zeitabhängigen Anisotropiemessungen und zeitabhängigen Stokes-Shift-Messungen hat dabei gezeigt, daß auf einer NanosekundenZeitskala vorrangig diffusive Rotations- und Wobble-Mechanismen zur Solvatation des Chromophors in einer eingeschränkten Lipidmembranumgebung beitragen. Die in der Literatur propagierte Stabilisierung des Chromophors durch eingeschränktes Wasser auf einer Nanosekunden-Zeitskala spielt zumindest in dem hier untersuchten DMPC/Laurdan/Wasser-System keine oder nur eine untergeordnete Rolle. Die Fluoreszenzkonversionsexperimente legen nahe, daß sich die Relaxation von Wassermolekülen innerhalb der Membran noch immer im Bereich von Pikosekunden abspielt, auch wenn die Dynamik im Vergleich zu reinem Wasser deutlich verlangsamt erscheint. Diese Verlangsamung ist vermutlich darauf zurückzuführen, daß der verwendete Chromophor durch seine tiefe Einbaulage eine stark eingeschränkte Wasserumgebung erfährt. Die tiefe Einbaulage erklärt auch die fehlende Empfindlichkeit auf den Vorphasenübergang der verwendeten Membran, welcher lediglich mit einer Umstrukturierung in der Kopfgruppenregion einhergeht. Die ausgeprägte Empfindlichkeit auf den Hauptphasenübergang ist vorrangig auf die stark erhöhte Bewegungsfreiheit des Chromophors durch das Schmelzen der Kettenregion in seiner unmittelbaren Umgebung zurückzuführen, was sich in allen spektroskopischen Untersuchungen bemerkbar macht. Darüberhinaus wurde das Auftreten eines Charge-Transfer-Komplexes des verwendeten Chromophors Laurdan in der flüssig-kristallinen Phase der Lipidmembran diskutiert.

Insgesamt gesehen stellen die hier vorgestellten Ergebnisse nach bestem Kenntnisstand die ersten Untersuchungen dar, die die Solvatationsdynamik eines Chromophors in eingeschränkten Lipidmembranumgebungen über einen Bereich von über fünf Größenordnungen eingehend charakterisieren konnten. Dabei kam an solchen Systemen zum ersten Mal die Technik der Fluoreszenzaufkonvertierung zum Einsatz. 



\section{Kapitel 6}

\section{Inverse Mizellen}

Im folgenden Kapitel werden die am Modellsystem der inversen Mizellen erhaltenen Meßdaten, sowie deren Auswertung vorgestellt. Die gewonnenen Ergebnisse werden eingehend diskutiert. Den Schwerpunkt stellt die Untersuchung der ultraschnellen Solvatationsdynamik in Abhängigkeit der Mizellengröße und im Vergleich zur Dynamik der reinen Wasserphase dar. Als Sonde für die Solvatation wurde der Farbstoff HIDCI verwendet.

Zunächst werden in Kapitel 6.1 die stationären Charakteristika der untersuchten Mikroemulsionen besprochen. Schwerpunkt sind die stationären spektroskopischen Eigenschaften des Farbstoffes HIDCI in inversen Mizellen unterschiedlicher Größe im Vergleich zu den entsprechenden Eigenschaften in der reinen Wasserphase. Kapitel 6.2 befaßt sich mit der Solvatationsdynamik des reinen Wassers, wie sie im Photon-Echo Peakshift Experiment bestimmt wurde, während sich Kapitel 6.3 auf die Solvatationsdynamik innerhalb der inversen Mizellen konzentriert. Die oszillatorischen Beiträge zur Peakshift-Funktion, die aus kohärent erzeugten Wellenpaketen resultieren, werden in Kapitel 6.4 näher behandelt. Die erhaltenen Erkenntnisse sind in Kapitel 6.5 zusammengefaßt.

\subsection{Stationäre Eigenschaften inverser Mizellen}

\subsubsection{Charakterisierung inverser Mizellen}

Wie bereits im einführenden Kapitel 3.3 deutlich wurde, eignen sich inverse Mizellen hervorragend um eingeschränkte Umgebungen zu studieren, da sich der Grad der Einschränkung problemlos über die Größe der Mizellen einstellen läßt. 
Daher wurden in dieser Arbeit gemäß den Ausführungen aus Kapitel 4.6.2 sechs Lösungen inverser Mizellen im System Isooktan/AOT/Wasser mit unterschiedlicher Zusammensetzung hergestellt und untersucht. Diese Mikroemulsionen werden vorrangig durch die Parameter $\mu$ und $\alpha$ charakterisiert, welche wie folgt definiert sind:

$$
\begin{aligned}
& \mu=\frac{\left[\mathrm{H}_{2} \mathrm{O}\right]}{[\mathrm{AOT}]} \quad \text { und } \\
& \alpha=\frac{m_{\mathrm{H}_{2} \mathrm{O}}}{m_{\mathrm{Gesamt}}} .
\end{aligned}
$$

Dabei bestimmt $\mu$ die Mizellengröße, während $\alpha$ in Beziehung zur Anzahl der in Lösung befindlichen Wassertröpfchen steht.

Damit sich in der organischen Phase überhaupt inverse Mizellen ausbilden können, muß sichergestellt sein, daß sich die hergestellten Lösungen innerhalb der sogenannten $\mathrm{L}_{2}$-Phase des untersuchten Systems befinden. In dieser Phase bilden sich Wasser-in-Öl-Mikroemulsionen aus, d.h. die für unsere Zwecke benötigten inversen Mizellen [101]. Die genauen Parameter und Zusammensetzungen der hergestellten Lösungen sind Tabelle $6.1 \mathrm{zu}$ entnehmen.

\begin{tabular}{|c|c|c|c|c|c|}
\hline $\boldsymbol{\mu}$ & $\boldsymbol{\alpha}$ & $\boldsymbol{O D}$ & $\boldsymbol{x}_{\text {Isooktan }}$ & $\boldsymbol{x}_{\text {AOT }}$ & $\boldsymbol{x}_{\text {Wasser }}$ \\
\hline 1 & 0.0202 & 0.1 & 0.652 & 0.174 & 0.174 \\
2 & 0.0400 & 0.2 & 0.551 & 0.150 & 0.299 \\
4 & 0.0939 & 0.5 & 0.305 & 0.139 & 0.556 \\
8 & 0.1059 & 0.5 & 0.429 & 0.063 & 0.508 \\
16 & 0.1132 & 0.5 & 0.483 & 0.030 & 0.487 \\
32 & 0.1172 & 0.5 & 0.508 & 0.015 & 0.477 \\
\hline
\end{tabular}

Tabelle 6.1: Zusammensetzung der untersuchten Mikroemulsionen.

Abbildung 6.1 stellt die Verhältnisse in einem ternären Phasendiagramm dar. Es ist $\mathrm{zu}$ beachten, daß es sich hier um eine Auftragung gegen Gewichtsprozente wt\% handelt. Das Diagramm wurde aus einer Arbeit von Kunieda et al. entnommen und gilt für $T=15^{\circ} \mathrm{C}$ [247]. Alle hergestellten Lösungen befinden sich danach innerhalb der $\mathrm{L}_{2}$-Phase und bilden entsprechend inverse Mizellen aus. Beim Herstellungsprozeß des AOT's verbliebene Verunreinigungen (vorrangig $\mathrm{Na}_{2} \mathrm{SO}_{4}$ ) verursachen jedoch innerhalb der $\mathrm{L}_{2}$-Phase eine Mischungslücke, welche in Abbildung 6.1 entsprechend gekennzeichnet ist und in der sich die Lösung mit $\mu=32$ befindet. Die Lösung entmischt sich im Kühlschrank (ca. $8^{\circ} \mathrm{C}$ ) und wird entsprechend trübe. Bei Zimmertemperatur erscheint die Lösung jedoch klar, so daß unter Meßbedingungen von einer Mizellenbildung ausgegangen werden kann. 


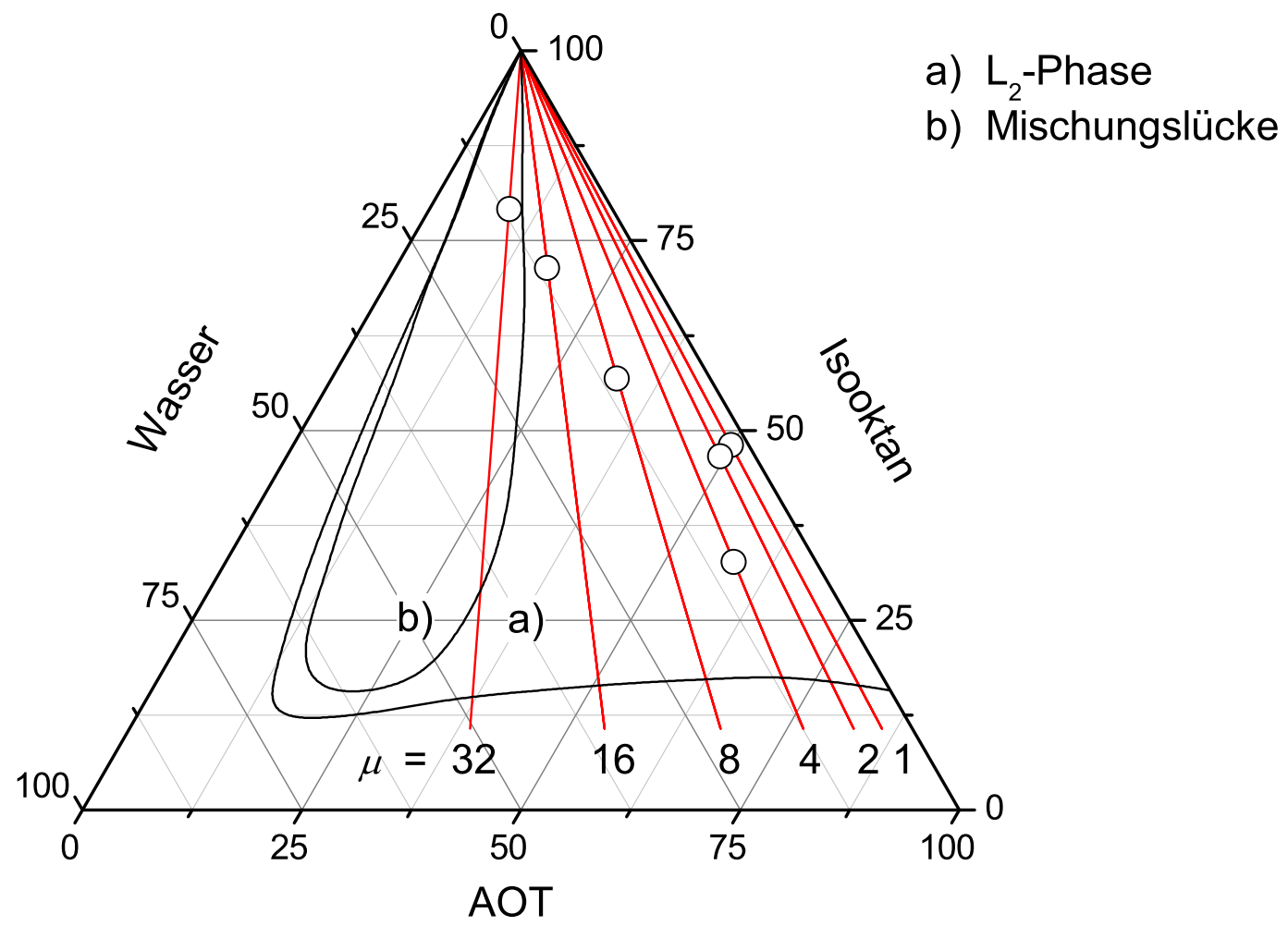

Abbildung 6.1: Ternäres Phasendiagramm des Systems Isooktan/AOT/Wasser in wt\%. Die $L_{2}$-Phase, die Mischungslücke und die Zusammensetzung der hergestellten Mikroemulsionen sind entsprechend markiert.

Trotz dieses Hinweises auf die Gültigkeit des Phasendiagramms für das hier diskutierte System, läßt sich nicht ausschließen, daß weitere Faktoren, wie z. B. die Reinheit des AOT's und der in der vorliegenden Arbeit im System befindliche Farbstoff, für die genaue Lage der in Abbildung 6.1 dargestellten Phasen eine Rolle spielt. Aufgrund der Klarheit der untersuchten Lösungen bei Zimmertemperatur kann jedoch für alle Proben von einer Mizellenbildung ausgegangen werden.

Der in ${ }^{1} \mathrm{H}$-Kernresonanzspektren beobachtete chemische Shift der Protonen in Abhängigkeit von $\mu$ im Isooktan/AOT/Wasser-System konnte von Maitra in direkte Beziehung zum mittleren Radius $r_{\mathrm{w}}$ des Wassernanotröpfchens in inversen Mizellen gesetzt werden [115]. Der Anteil von gebundenem zu freiem Wasser $P_{\mathrm{b}}$ wurde unter Voraussetzung einer konstanten Wasserdichte über den Querschnitt des Wassernanotröpfchens wie folgt berechnet:

$$
P_{\mathrm{b}}=1-\left(1-\frac{d}{r_{\mathrm{w}}}\right)^{3}
$$


wobei $d$ die Dicke der gebundenen Wasserschicht bezeichnet, welche als $d=4 \AA$ angenommen wurde.

Weiterhin ist die mittlere Anzahl von Wassermolekülen $n_{\mathrm{w}}$ in den untersuchten Mizellen für eine Diskussion des Grades der Einschränkung von Interesse [115, 125]. Alle Daten sind in Tabelle 6.2 zusammengefaßt.

\begin{tabular}{|c|c|c|c|}
\hline $\boldsymbol{\mu}$ & $\boldsymbol{r}_{\mathrm{w}} / \AA$ & $\boldsymbol{P}_{\mathrm{b}}$ & $\boldsymbol{n}_{\mathrm{w}}$ \\
\hline 1 & 6.0 & 0.96 & 20 \\
2 & 7.5 & 0.90 & 50 \\
4 & 10.5 & 0.76 & 140 \\
8 & 16.6 & 0.56 & 600 \\
16 & 28.7 & 0.36 & $3.5 \cdot 10^{3}$ \\
32 & 53.0 & 0.21 & $21.0 \cdot 10^{3}$ \\
\hline
\end{tabular}

Tabelle 6.2: Parameter inverser Mizellen in Abhängigkeit von $\mu$ nach Maitra [115].

\subsubsection{Stationäre spektroskopische Eigenschaften}

Abbildung 6.2 stellt die normierten stationären Absorptions- und Emissionsspektren von HIDCI in reinem Wasser den entsprechenden Spektren des gleichen Farbstoffes in inversen Mizellen gegenüber. Repräsentativ sind Spektren für $\mu=1,4$ und 32 dargestellt. Alle Spektren wurden bei Zimmertemperatur gemessen. Die Emissionsspektren wurden bei einer Anregungswellenlänge von $600 \mathrm{~nm}$ im rechten Winkel zur Anregung (RA) aufgenommen.

Die Bandenformen für Absorption und Emission von HIDCI in Wasser sind sehr ähnlich, lediglich die Nebenbande ist im Emissionsspektrum deutlich weniger ausgeprägt. Das Absorptionsspektrum besitzt bei $635.9 \mathrm{~nm}\left(15726 \mathrm{~cm}^{-1}\right)$ sein Maximum, während das Fluoreszenzspektrum bei $659.4 \mathrm{~nm}\left(15165 \mathrm{~cm}^{-1}\right)$ maximal ist. Das entspricht einem stationären Stokes-Shift von $561 \mathrm{~cm}^{-1}$. Anpassungen der Hauptbanden mit einer Gaußfunktion zeigen Halbwertsbreiten von $770 \mathrm{~cm}^{-1}$ (Absorption) bzw. $650 \mathrm{~cm}^{-1}$ (Emission).

Beim Übergang von der reinen Wasserphase zu den inversen Mizellen fällt zunächst die prinzipielle Ähnlichkeit der Spektrenformen und der vergleichbaren spektralen Lage der Spektren auf. Das ist ein Indiz dafür, daß HIDCI in der Wasserphase der Mikroemulsionen gelöst ist. Die Spektren sind jedoch im Gegensatz zu reinem Wasser allesamt leicht zu geringeren Wellenzahlen verschoben. Diese Rotverschiebung der Absorptions- und Emissionsspektren ist ein Hinweis auf die polarere Umgebung der Wasserphase in inversen Mizellen, die durch die geladene 


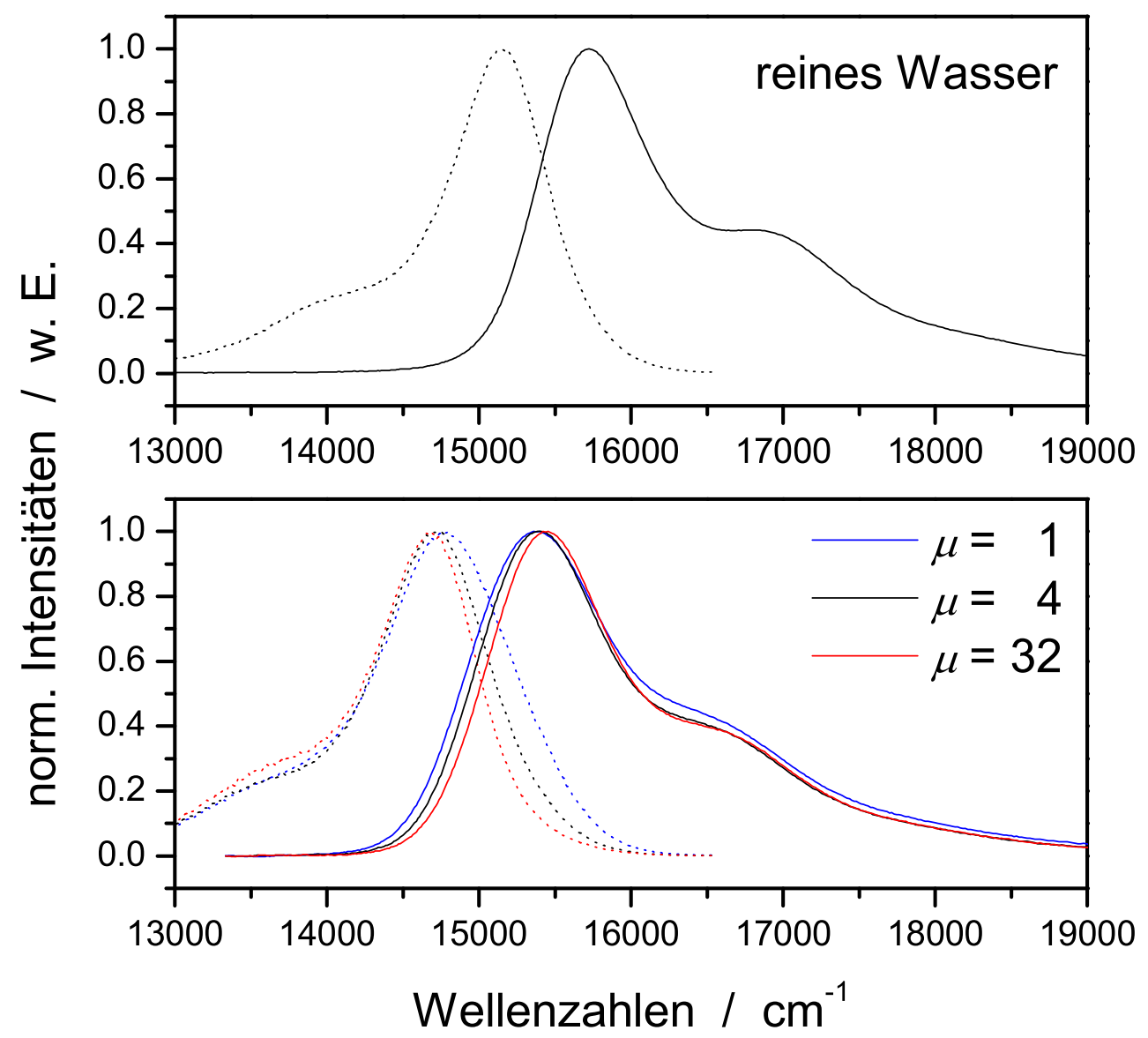

Abbildung 6.2: Absorptions- (durchgezogene Linien) und Emissionsspektren (gestrichelte Linien) für HIDCI in reinem Wasser (oben) und inversen Mizellen mit $\mu=1,4$ und 32 (unten).

Kopfgruppe des AOT's und der in Lösung befindlichen Gegenionen verursacht wird. Ein ähnlicher Effekt wird auch für den an einer Lipidmembran/WasserGrenzschicht lokalisierten Farbstoff C18-DiD beobachtet, dessen Chromophor mit dem hier untersuchten HIDCI identisch ist [50].

Bei genauer Betrachtung werden Unterschiede in den stationären spektroskopischen Eigenschaften inverser Mizellen unterschiedlicher Größe deutlich. Die Absorptionsspektren zeigen eine geringfügige Rotverschiebung beim Übergang zu kleineren Mizellen bei gleichzeitiger Zunahme ihrer Breite. Bei den Emissionsspektren ist der Effekt stärker ausgeprägt. Hier wird allerdings eine Blauverschiebung beim Übergang zu kleineren Mizellen beobachtet, während die Breite ebenfalls zunimmt. Daraus folgt, daß die Solvatationsreorganisationsenergie mit kleiner werdender Mizellengröße etwas abnimmt. Die Verschiebungen werden insbesondere beim Übergang zu den sehr geringen Mizellengrößen ( $\mu=1$ bzw. 2) 
deutlich. Auffällig ist, daß selbst die Spektren der größten Mizellen Unterschiede zu den Spektren des reinen Wassers aufweisen, obschon der Wassereinschluß dieser Mizellen aus mehr als 20000 Wassermolekülen besteht und daher weitestgehend repräsentativ für die reine Wasserphase sein sollten (siehe Tabelle 6.2). Diese Diskrepanz kann vermutlich dadurch erklärt werden, daß der Wassereinschluß der inversen Mizellen im Vergleich zu reinem Wasser eine stark ionische Lösung darstellt. Da sich die Gegenionen jedoch größtenteils in der Nähe der Grenzschicht aufhalten, liegt es nahe anzunehmen, daß der Chromophor HIDCI sich ebenfalls an der Grenzschicht anlagert und sich nicht in der freien Wasserphase der Mizellen befindet. Für diese Interpretation spricht auch die Ladung des Chromophors, die gegensätzlich zur Ladung der Kopfgruppen des AOT's ist. Von Levinger et al. wurde im gleichen System eine vergleichbare Lokalisierung des Chromophors Coumarin 343 an der Grenzschicht durch Anisotropiemessungen nachgewiesen [105]. Die stationären Spektren allein können allerdings nicht als ein eindeutiger Beleg für die Anlagerung des Chromophors an der Grenzschicht gewertet werden. Die genaue Position der Farbstoffsonde innerhalb der inversen Mizellen bedarf daher weiterer Untersuchungen.

Für die inversen Mizellen wird ein Stokes-Shift im Bereich von $627 \mathrm{~cm}^{-1}$ für $\mu=1$ bis $765 \mathrm{~cm}^{-1}$ für $\mu=32$ beobachtet. Damit ist die Solvatationsreorganisationsenergie in inversen Mizellen zwar etwas größer als in der reinen Wasserphase, die Verhältnisse sind dennoch vergleichbar. Bemerkenswert ist jedoch, daß der beobachtete Stokes-Shift in reinem Wasser mehr den kleineren als den größeren Mizellen ähnelt. Wiederum könnten dafür die ionischen Verhältnisse innerhalb des Wassereinschlusses der inversen Mizellen verantwortlich sein.

\subsection{Solvatationsdynamik in Wasser}

Zur Charakterisierung der Solvatationsdynamik von HIDCI in reinem Wasser werden in diesem Kapitel zeitintegrierte 3-Puls Photon-Echo Peakshift Messungen bei $T=25^{\circ} \mathrm{C}$ vorgestellt. Die Meßprozedur folgt dabei Kapitel 4.5.4. Die Zuordnung der beobachteten Zeitskalen zu bestimmten molekularen Freiheitsgraden des Wassers folgt im Wesentlichen den Arbeiten von Vöhringer und Mitarbeitern und wird daher im folgenden nur knapp dargestellt. Eine ausführliche Diskussion ist der entsprechenden Literatur zu entnehmen $[27,50,143]$. Auf eine weiterreichende Interpretation der Solvatationsdynamik im Modell der Brown'schen Oszillatoren wird im folgenden nicht näher eingegangen (siehe dazu [79]). 
Eine repräsentative Auswahl normierter 3-Puls Photon-Echos mit zugehörigen Autokorrelationen sind für verschiedene Verzögerungszeiten $t_{23}$ in Abbildung 6.3 dargestellt.

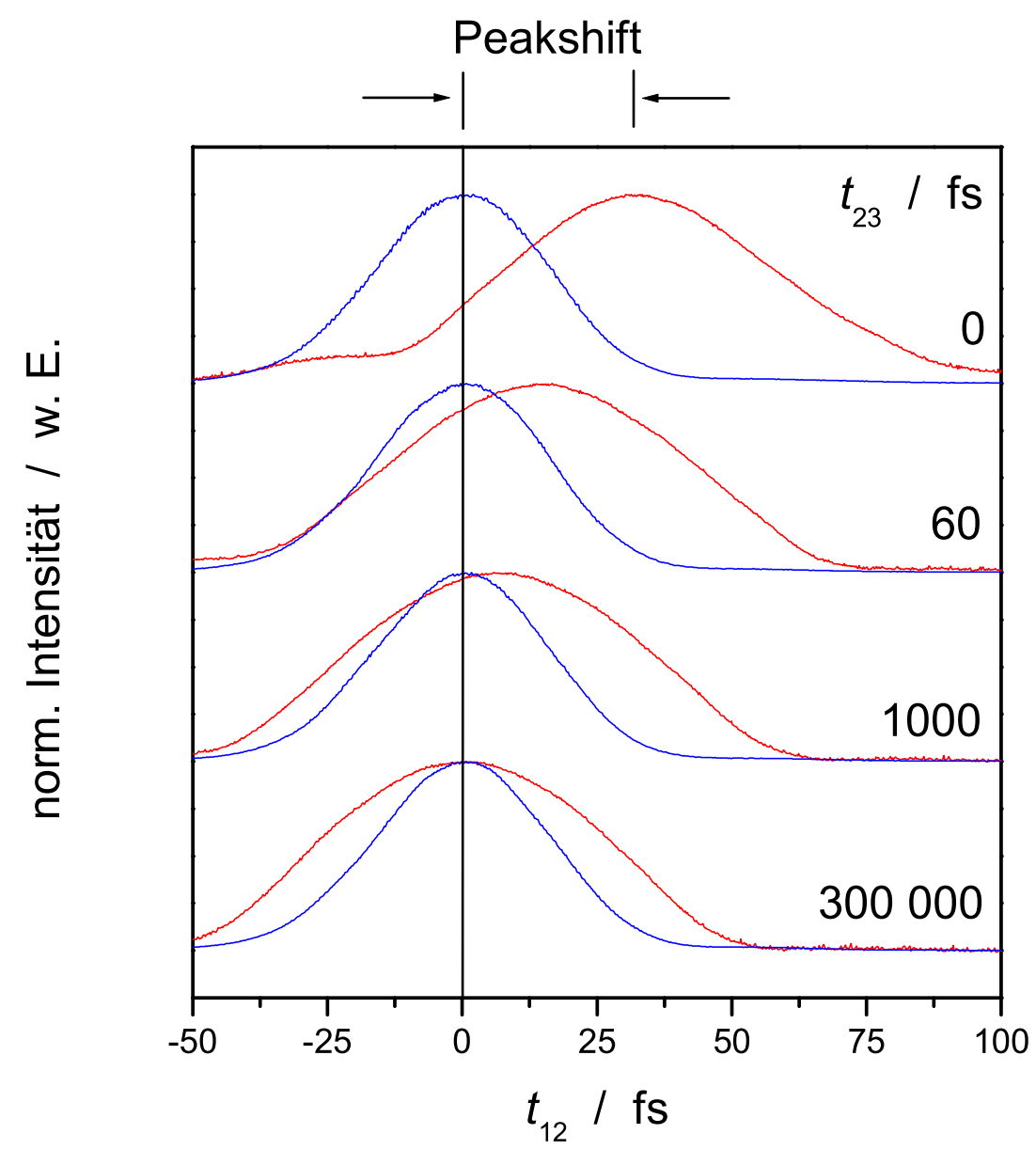

Abbildung 6.3: Auswahl repräsentativer 3-Puls Photon-Echos (rot) und Autokorrelationen (blau) bei verschiedenen Verzögerungszeiten $t_{23}$ für HIDCI in reinem Wasser.

Um den Peakshift bei einer festen Verzögerungszeit $t_{23} \mathrm{zu}$ bestimmen, wurden die Daten zunächst geglättet und an ein Polynom dritten Grades angepaßt. Dabei wurden lediglich die oberen $20 \%$ der detektierten Signale berücksichtigt (siehe dazu auch Kapitel 4.5.4). Es ist bereits aus Abbildung 6.3 ersichtlich, daß der Peakshift mit wachsender Verzögerungszeit $t_{23}$ abnimmt. Der auf diese Weise erhaltene Peakshift in Abhängigkeit von $t_{23}$ ist in Abbildung 6.4 in halblogarithmischer Auftragung dargestellt. Es sei an dieser Stelle nochmals angemerkt, daß die in der Boxcargeometrie aufgenommenen Messungen eine gewisse Ungenauigkeit bezüglich des absoluten Peakshifts aufweisen. 


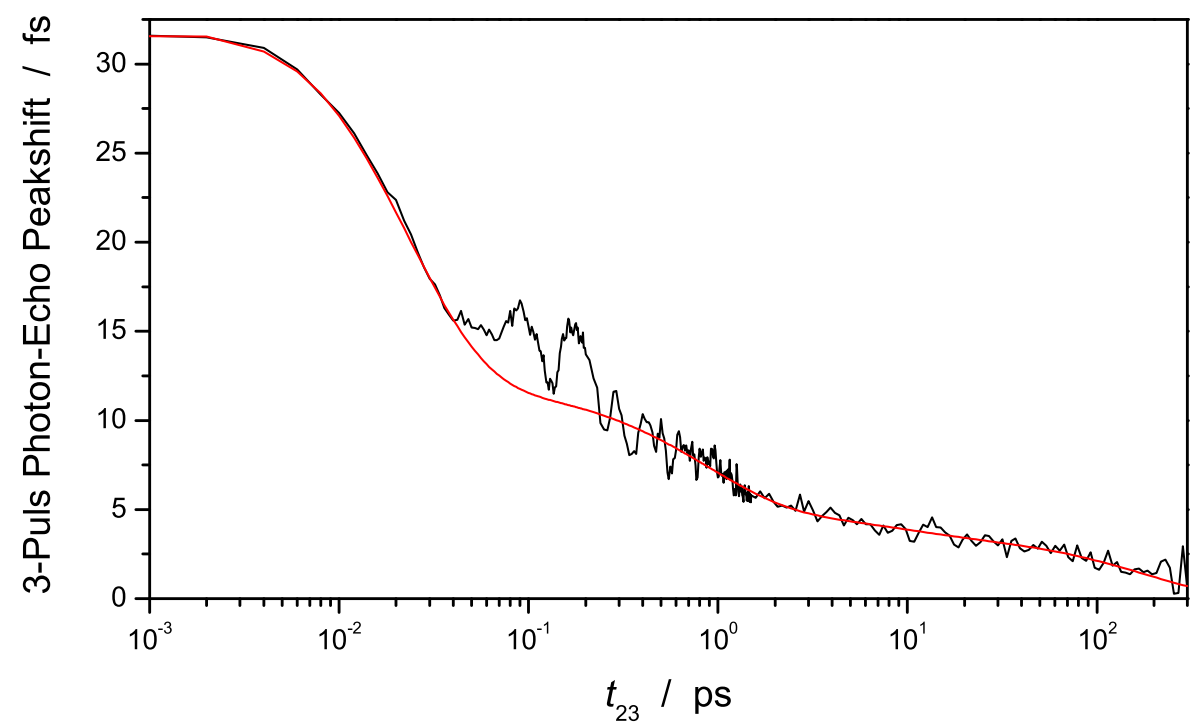

Abbildung 6.4: Peakshift-Funktion $S\left(t_{23}\right)$ von HIDCI in reinem Wasser bei $T=25^{\circ} \mathrm{C}$ mit Anpassung nach Gleichung 6.4.

Die Peakshift-Funktion $S\left(t_{23}\right)$ wurde mit einer sogenannten Kubo-Funktion für den Inertialteil bis ca. $100 \mathrm{fs}$ und einer Summe aus drei Exponentialfunktionen für die langsameren Zeitkomponenten angepaßt:

$$
\begin{aligned}
I\left(t_{23}\right)= & A_{1} \exp \left(-\Delta^{2} \tau_{1}\left[t_{23}-\tau_{1}\left(1-\exp \left(-\frac{t_{23}}{\tau_{1}}\right)\right)\right]\right) \\
& +\sum_{i=2}^{4} A_{i} \exp \left(-\frac{t_{23}}{\tau_{i}}\right) .
\end{aligned}
$$

Darin bezeichnen $\tau_{1}-\tau_{4}$ die Zeitkonstanten mit den zugehörigen Amplituden $A_{1}-A_{4}$. Im Vergleich zu früheren Arbeiten wurde für den Inertialteil anstatt einer Gaußfunktion eine Kubo-Funktion gewählt, da sich eine Anpassung mit einer Gaußfunktion als unbefriedigend erwies.

Eine Kubo-Funktion ist in der Lage zwischen gaußförmiger und exponentieller Dynamik zu interpolieren (siehe dazu Kubo et al. [248]). Im Grenzfall $t \ll \tau_{1}$ geht die Kubo-Funktion in eine Gaußfunktion über:

$$
\phi\left(t_{23}\right)=A_{1} \exp \left(-\frac{1}{2} \Delta^{2} t_{23}^{2}\right)
$$


Das Spektrum ist entsprechend ebenfalls gaußförmig und stellt damit den inhomogenen Grenzfall in der Bloch'schen Beschreibung dar. Der Anpassungsparameter $\Delta$ legt das inhomogene Limit fest.

Für $t \gg \tau_{1}$ wird eine Exponentialfunktion erhalten:

$$
\phi\left(t_{23}\right)=A_{1} \exp \left(-\Delta^{2} \tau_{1} t_{23}+\Delta^{2} \tau_{1}^{2}\right) .
$$

Deren Spektrum ist lorentzförmig und repräsentativ für homogene Linienverbreiterungsmechanismen. In der Frequenzdomäne entspricht die Kubo-Funktion also einem Übergang von einer gänzlich inhomogenen (gaußförmigen) in eine gänzlich homogene (lorentzförmige) Linienverbreiterung. Die Peakshift-Funktion sollte daher korrekt mit einer Summe aus Kubo-Funktionen dargestellt werden. Da das Abklingverhalten des Peakshifts auf mittleren bis langen Zeitskalen aber sehr gut mit einfachen Exponentialfunktionen wiedergegeben werden kann, wurde der Anpassung mit lediglich einer Kubo-Funktion und drei Exponentialfunktionen der Vorzug gegeben. Dabei ist zu beachten, daß die Zeitkonstante $\tau_{1}$ der KuboFunktion nicht unmittelbar mit Zeitkonstanten verglichen werden kann, wie sie für einfache Gauß- bzw. Exponentialfunktionen erhalten werden. Soll dennoch ein direkter Vergleich erfolgen, so eignet sich die Größe $\left(\Delta^{2} \tau_{1}\right)^{-1}$. Die zur Anpassung der Peakshift-Funktion von HIDCI in Wasser verwendeten Anpassungsparameter nach Gleichung 6.4 sind in Tabelle 6.3 zusammengefaßt.

\begin{tabular}{|c|c|c|c|c|}
\hline $\boldsymbol{i}$ & $\boldsymbol{A}_{\boldsymbol{i}} / \mathbf{f s}$ & $\boldsymbol{A}_{\boldsymbol{i}} / \sum \boldsymbol{A}_{\boldsymbol{i}}$ & $\boldsymbol{\tau}_{\boldsymbol{i}} / \mathbf{p s}$ & $\boldsymbol{\Delta} / \mathbf{p s}^{-\mathbf{1}}$ \\
\hline 1 & 19.8 & $62.1 \%$ & 0.0046 & 100 \\
2 & 7.0 & $21.9 \%$ & 0.85 & - \\
3 & 1.4 & $4.4 \%$ & 7.5 & - \\
4 & 3.7 & $11.6 \%$ & 180 & - \\
\hline
\end{tabular}

Tabelle 6.3: Anpassungsparameter nach Gleichung 6.4 für die Peakshift-Funktion in reinem Wasser.

Die ermittelten Zeitkonstanten aus Tabelle 6.3 lassen sich wie folgt aufschlüsseln:

\section{Zeitkonstante $\tau_{1}$}

Der ultraschnelle Anteil der Peakshift-Funktion $S\left(t_{23}\right)$ unterhalb ca. 100 fs wird sehr gut durch eine Kubo-Funktion wiedergegeben (siehe Abbildung 6.4). Daß sich dieser Inertialteil mit einer Gaußfunktion [36] nur unbefriedigend beschreiben ließ, weist darauf hin, daß die Separation in homogene und inhomogene Linienverbreiterungsmechanismen nach den optischen Bloch-Gleichungen in Flüssigkeiten kein adäquates Konzept darstellt und nicht länger aufrecht erhalten werden kann. 
Als Ursache der ultraschnellen Dynamik wurden von Jimenez et al. die Librationsmoden der Wassermoleküle in den ersten Hydratationschalen des Chromophors angeführt [24]. Ob dies jedoch tatsächlich zutrifft ist fragwürdig, da die schnellsten intermolekularen Moden des Wassers eine Schwingungsperiode von 67 fs aufweisen. Auch Erklärungsansätze anderer Arbeitsgruppen mit Hilfe molekulardynamischer Simulationen [71, 73, 249] und dielektrischen Kontinuumstheorien [74] führten zu keiner befriedigenden Antwort.

Beim zeitintegrierten 3-Puls Photon-Echo Peakshift Experiment muß weiterhin bedacht werden, daß der schnelle Anteil in keiner direkten Beziehung zur SystemBad-Korrelationsfunktion steht, wie in Kapitel 2.3 ausführlich dargestellt wurde. Der beobachtete schnelle Anteil kann sicherlich nicht mehr zum langsamen Anteil der Korrelationsfunktion gezählt werden. Aus diesem Grund wird von einer weiteren Diskussion der Ursache der ultraschnellen Komponente abgesehen.

\section{Zeitkonstante $\tau_{2}$}

Auf Zeitskalen von ca. $100 \mathrm{fs}$ bis 3 ps weist die Peakshift-Funktion ein ausgeprägtes exponentielles Abklingen mit einer Zeitkonstanten von $\tau_{2}=0.85 \mathrm{ps}$ auf, welche mit einer Genauigkeit von ca. \pm 0.2 ps bestimmt werden kann. Die ermittelte Zeitkomponente kann mit einer Relaxation von übergedämpften, kollektiven Translationsmoden des Wassers interpretiert werden, wie ein Vergleich mit Messungen des optischen Kerr-Effektes in Wasser [45] und depolarisierten Streuexperimenten [250] nahelegt. Desweiteren wird dieser Zeitbereich von kritisch gedämpften, gehinderten Translationsmoden des Wassers bestimmt, welche die Streckung und Biegung von Wasserstoffbrückenbindungen in Wasserclustern unterschiedlicher Größe beschreiben. Diese Moden sind für das zufällige Wasserstoffbrückennetzwerk, wie es nur in reinem Wasser vorkommt, außerordentlich charakteristisch. Computer-Simulationen von Marcus et al. stützen diese Interpretation [251,252]. Nach Walrafen liegt die Herkunft dieser Moden in lokalen Schwingungen der Flüssigkeit wie sie auch in Festkörpern vorkommen [55]. Deren Frequenzen stimmen mit den transversal akustischen (TA) und longitudinal akustischen (LA) Phononen-Frequenzen von amorphen und kristallinem Eis überein (TA: $65-70 \mathrm{~cm}^{-1}$, LA: $164-181 \mathrm{~cm}^{-1}$ ).

Die Komponente mit einer Zeitkonstanten um 1 ps scheint generell für die Solvatation in reinem Wasser dominierend zu sein. Sie wurde in einer Vielzahl weiterer Messungen zur Dynamik des Wassers beobachtet [45, 66, 253, 254, 255, 256, 257]. Ähnliche Zeitkonstanten wurden auch in Fluoreszenzaufkonvertierungsexperimenten von Coumarinen in Wasser $[24,49,258]$ und einer 3PEPS-Studie des Chromophors Eosin Y in Wasser gefunden [38]. 
Im Bereich von ca. $50 \mathrm{fs}$ bis 1 ps fallen darüberhinaus oszillatorische Beiträge zur Peakshift-Funktion auf. Sie resultieren aus intramolekularer Wellenpaketdynamik des Chromophors, die durch die erzeugte Kohärenz bei optischer Anregung herbeigeführt wurde. Von Wiersma et al. wurde gezeigt, daß diese Oszillationen in keinerlei Beziehung zu strukturellen Fluktuationen in der Mikroumgebung des Chromophors stehen [29,38, 259].

Eine Schwingungsanalyse an den oszillatorischen Beiträgen der PeakshiftFunktion wurde von Bürsing am gleichen System wie in der vorliegenden Arbeit durchgeführt [27]. Erwartungsgemäß zeigte sich, daß die Oszillationen auf die Schwingungsstruktur des Chromophors HIDCI zurückgeführt werden kann und prinzipiell die gleiche Information wie post-resonante Raman-Spektren und Transient-Grating-Messungen enthält. Da die Qualität der Schwingungsanalyse mit den letzteren Techniken aber ungleich besser ist, wird hier von einer weitergehenden Analyse der oszillatorischen Beiträge im Peakshift Experiment abgesehen. Eine Analyse der Schwingungsstruktur anhand der Transient-Grating-Messungen folgt in Kapitel 6.4.

\section{Zeitkonstante $\tau_{3}$}

Das exponentielle Abklingen der Peakshift-Funktion mit einer Zeitkonstanten von $\tau_{3}=7.5$ ps dominiert den Zeitbereich zwischen 3 und 50 ps. Die geringe Amplitude erlaubt lediglich eine Bestimmung der Zeitkonstanten von \pm 2 ps. Die Zeitkonstante stimmt sehr gut mit der Korrelationszeit der diffusiven Reorientierung individueller Wassermoleküle überein, wie sie aus Messungen der dielektrischen Relaxation (DR) [66], zeitaufgelösten THz-Experimenten [255,256] und longitudinalen NMR-Relaxationszeiten [260,261,262] bestimmt wurde. Die gleiche Dynamik wurde auch bei der Messung des Raman-induzierten optischen Kerr-Effektes (OKE) beobachtet [45]. Dort wurde gezeigt, daß die dielektrische Relaxationszeit $\tau_{\mathrm{d}}$, die in NMR-Experimenten erhaltene Spin-Gitter Relaxationszeit $R_{1}$ und die langsamste Zeitkonstante der OKE-Messungen alle die gleiche diffusive Rotationsdynamik einzelner Wassermoleküle beschreiben.

\section{Zeitkonstante $\tau_{4}$}

Die langsamste Komponente weist eine Zeitkonstante von $\tau_{3}=180$ ps auf und deutet darauf hin, daß selbst auf diesen langen Zeitskalen noch Inhomogenitäten im System vorhanden sind. Aufgrund der beschriebenen Ungenauigkeiten in der Festlegung des absoluten Peakshifts in der Boxcar-Geometrie kann diese Zeitkonstante lediglich mit einer Genauigkeit von $\pm 50 \mathrm{ps}$ bestimmt werden. Diese langsame Zeitkomponente ist vermutlich auf die kollektive Rotationsdiffusion des Lösungsmittels zurückzuführen [50]. Auf ähnlichen Zeitskalen findet aber auch 
die Rotationsdiffusion des verwendeten Chromophors statt. Eine genauere Zuordnung ist aufgrund der Ungenauigkeit der bestimmten Zeitkonstante und der unvollständigen Information bezüglich der kollektiven Rotationsmoden des Wassers an dieser Stelle nicht möglich.

\subsection{Solvatationsdynamik in inversen Mizellen}

Für die inversen Mizellen unterschiedlicher Größe wurden gemäß den Ausführungen in Kapitel 6.2 analoge 3-Puls Photon-Echo Messungen mit Hilfe der Farbstoffsonde HIDCI bei $T=25^{\circ} \mathrm{C}$ durchgeführt und deren Peakshift-Funktionen $S\left(t_{23}\right)$ ermittelt. Die erhaltenen Peakshift-Funktionen für $\mu=1,2$ und 4 sind in Abbildung 6.5 dargestellt, während Abbildung 6.6 die entsprechenden Daten für $\mu=8,16$ und 32 enthält.

Die Peakshift-Funktionen wurden analog den Messungen in reinem Wasser mit einer Kubo-Funktion für den Inertialteil und einer Summe aus drei Exponentialfunktionen für die langsameren Komponenten gemäß Gleichung 6.4 angepaßt. Die ungenau zu bestimmende langsamste Komponente wurde auf 180 ps entsprechend der Zeitkonstanten des reinen Wassers festgesetzt (siehe Kapitel 6.2), womit alle Datensätze sehr gut angepaßt werden konnten. Damit sind die hier vorgestellten Experimente lediglich auf die ultraschnellen Zeitkomponenten der Solvatationsdynamik sensitiv. Die in heterogenen Umgebungen auftretenden langsamen Zeitkomponenten wie sie beispielsweise im zeitkorrelierten Einzelphotonenzählexperiment ermittelt werden (siehe dazu Kapitel 3.3.2) sind somit nicht Gegenstand der hier vorgestellten Untersuchungen.

Die so erhaltenen Anpassungsparameter zeigt Tabelle 6.4. Zum direkten Vergleich sind in dieser Tabelle die Parameter für reines Wasser ebenfalls aufgeführt. Die Qualität der Messungen in inversen Mizellen ist mit derjenigen der reinen Wassermessung vergleichbar. Die Abhängigkeiten der ermittelten Zeitkonstanten und relativen Amplituden von $\mu$ sind in Abbildung 6.7 graphisch verdeutlicht.

Generell zeigt sich, daß in inversen Mizellen ähnliche Zeitkonstanten wie in der reinen Wasserphase gefunden werden: In allen Mizellen werden sowohl ein ausgeprägter Inertialteil, als auch die zwei charakteristischen Exponentialzeiten des Wassers um 1 bzw. 10 ps gefunden. Die Beobachtung dieser für reines Wasser charakteristischen Zeitkomponenten ist ein eindeutiger Beweis dafür, daß sich der Chromophor innerhalb der Wasserphase der inversen Mizellen befindet. Die gefunden Zeitkomponenten zeigen nur eine geringe bzw. keine Abhängigkeit mit wachsender Einschränkung der Umgebung, d. h. kleiner werdender Mizellengröße. Dies wird im folgenden eingehender diskutiert. 


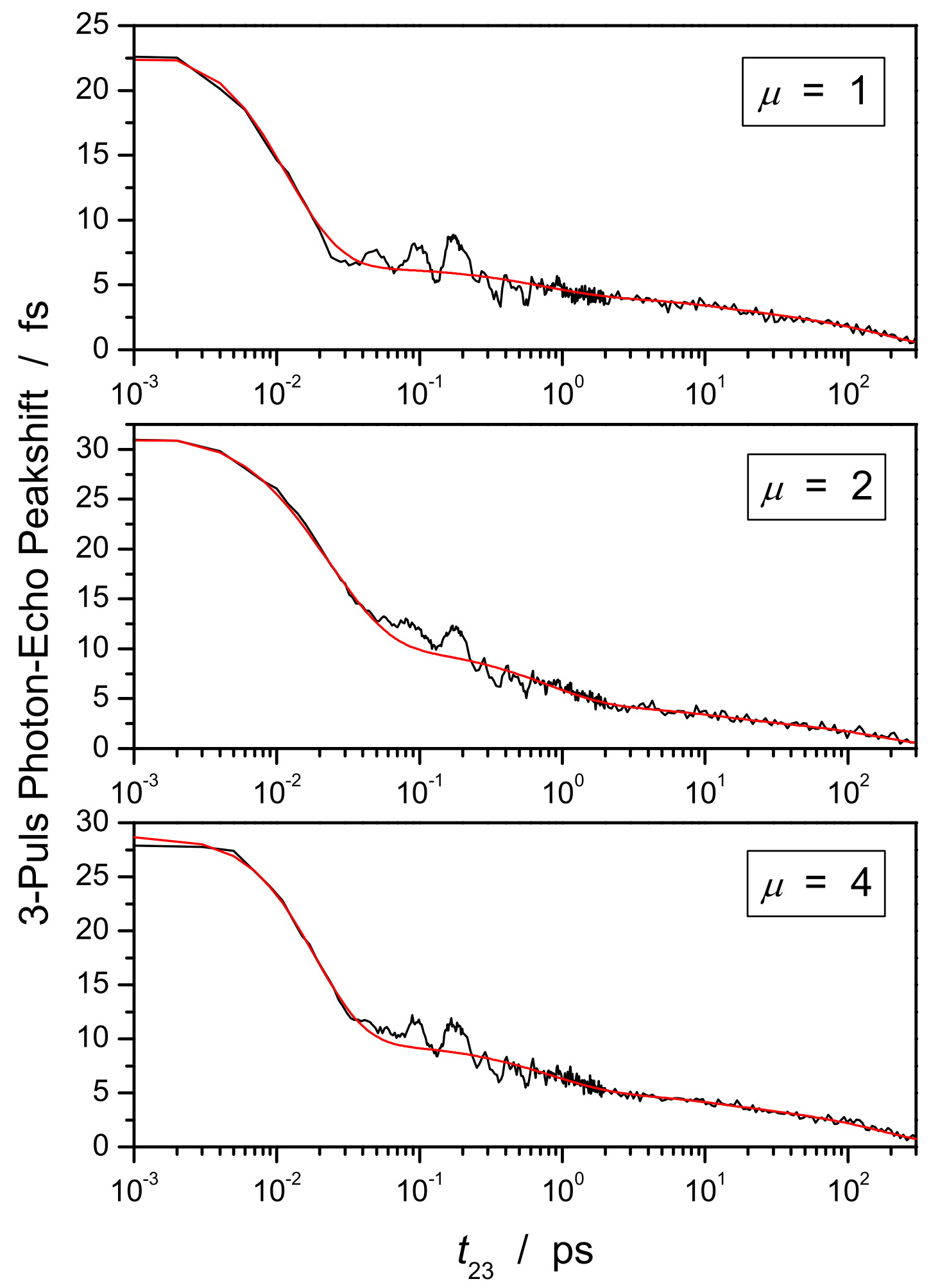

Abbildung 6.5: Peakshift-Funktionen $S\left(t_{23}\right)$ für inverse Mizellen mit $\mu=1,2$ und 4 mit Anpassungen nach Gleichung 6.4. 


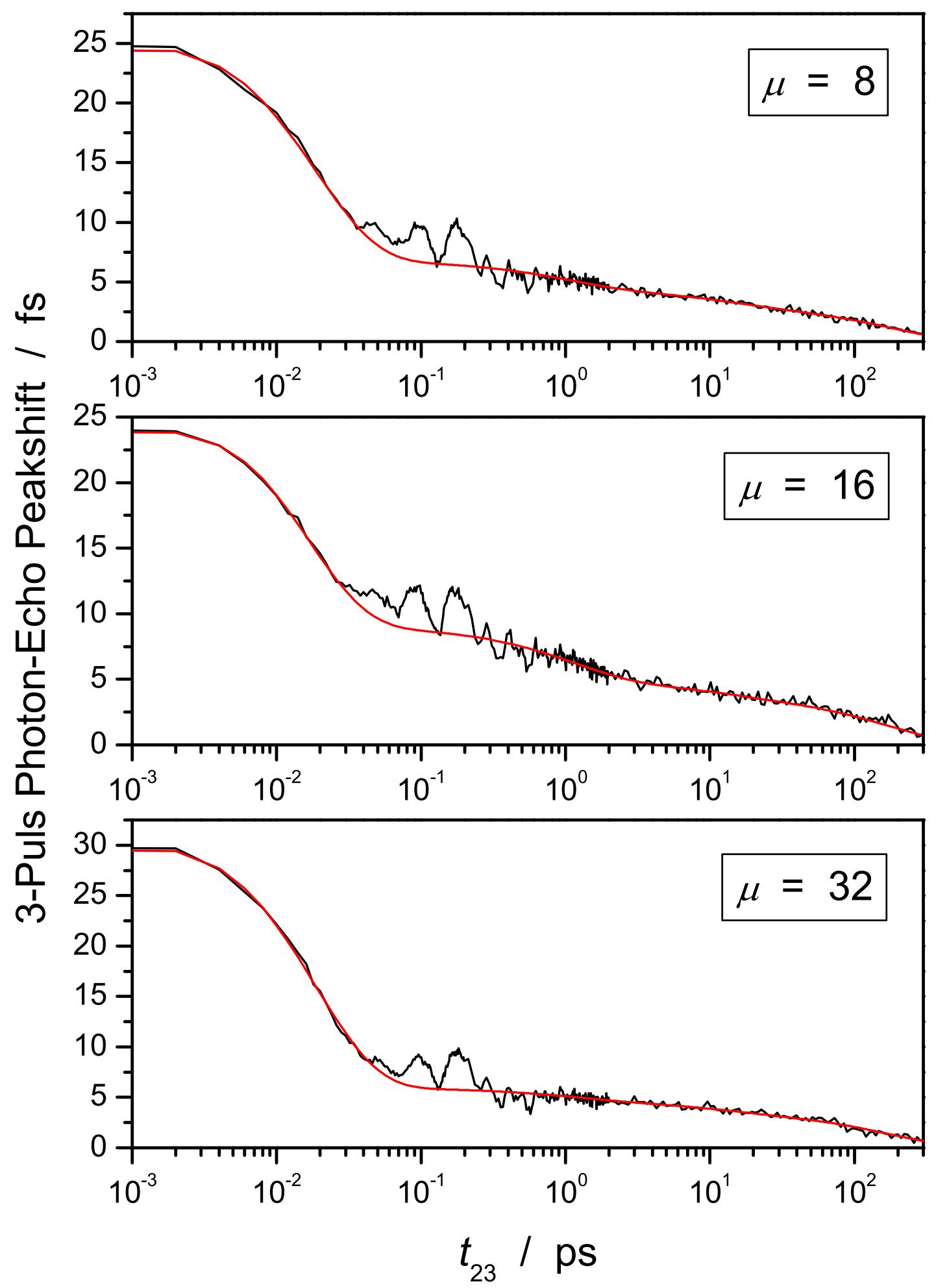

Abbildung 6.6: Peakshift-Funktionen $S\left(t_{23}\right)$ für inverse Mizellen mit $\mu=8,16$ und 32 mit Anpassungen nach Gleichung 6.4. 


\begin{tabular}{|c|c|c|c|c|c|c|c|}
\hline$\frac{n}{2}$ & $\stackrel{\infty}{\infty}$ & $\underset{\infty}{\infty}$ & $\underset{\infty}{\infty}$ & $\stackrel{\infty}{\infty}$ & $\stackrel{\infty}{\infty}$ & $\underset{\infty}{\infty}$ & $\stackrel{\infty}{-1}$ \\
\hline 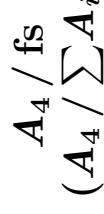 & 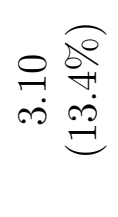 & 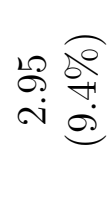 & 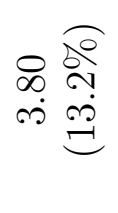 & 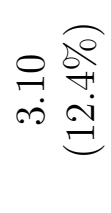 & 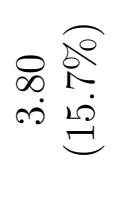 & 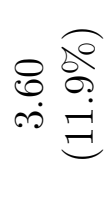 & 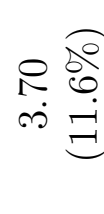 \\
\hline$\frac{n}{2}$ & $\stackrel{\sim}{\sim}$ & 그 & 二 & $\stackrel{\sim}{\sim}$ & 가 & 二 & $\stackrel{L}{\Lambda}$ \\
\hline 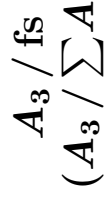 & 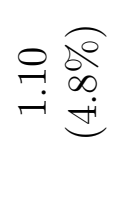 & 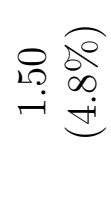 & 号 & 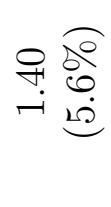 & 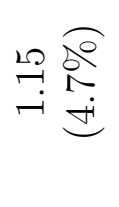 & 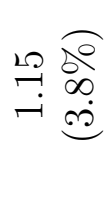 & 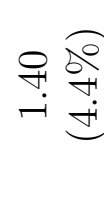 \\
\hline$\frac{n}{n}$ & $\stackrel{\sim}{\circ}$ & $\begin{array}{l}\stackrel{10}{N} \\
0\end{array}$ & $\stackrel{\infty}{0}$ & $\stackrel{0}{r}$ & $\stackrel{\longrightarrow}{\longrightarrow}$ & $\stackrel{\longrightarrow}{-}$ & $\begin{array}{l}20 \\
\infty \\
0\end{array}$ \\
\hline 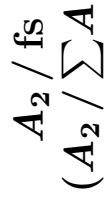 & 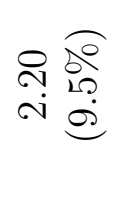 & 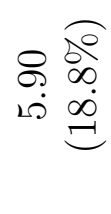 & 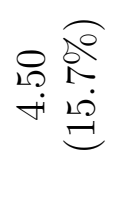 & 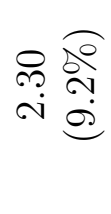 & 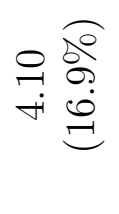 & 尺 & 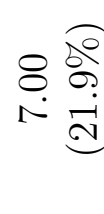 \\
\hline$\frac{n}{2}$ & $\begin{array}{l}\stackrel{2}{\mathscr{8}} \\
\stackrel{8}{0} \\
\stackrel{0}{0}\end{array}$ & $\begin{array}{l}\stackrel{0}{\mathscr{Q}} \\
\stackrel{8}{0} \\
\stackrel{0}{0}\end{array}$ & $\begin{array}{l}\stackrel{p}{P} \\
\stackrel{8}{0} \\
\stackrel{0}{0}\end{array}$ & $\begin{array}{l}\stackrel{H}{\Im} \\
\stackrel{8}{0} \\
\stackrel{0}{0}\end{array}$ & $\begin{array}{l}\infty \\
\stackrel{\infty}{8} \\
\stackrel{0}{0}\end{array}$ & 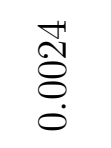 & $\begin{array}{l}0 \\
\stackrel{1}{8} \\
8 \\
0\end{array}$ \\
\hline 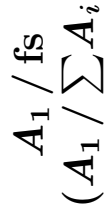 & 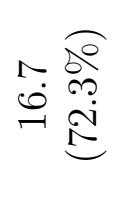 & 글 & 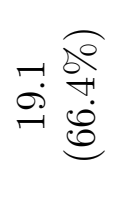 & 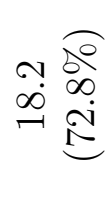 & 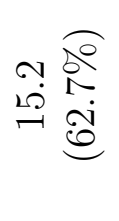 & 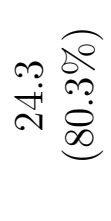 & 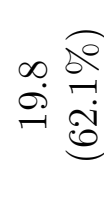 \\
\hline$\frac{i_{1}}{n^{2}}$ & $\stackrel{尺}{R}$ & $\stackrel{\leftrightarrow}{\oplus}$ & $\underset{-}{\varrho}$ & 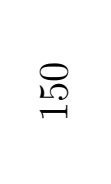 & $\stackrel{\text { คి }}{\rightarrow}$ & 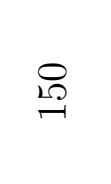 & $\underset{-}{\stackrel{-}{2}}$ \\
\hline$\vec{z}$ & $\neg$ & N & $\nabla$ & $\infty$ & $\stackrel{0}{-1}$ & ஸै & 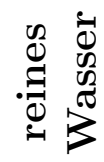 \\
\hline
\end{tabular}

Tabelle 6.4: Anpassungsparameter nach Gleichung 6.4 für die PeakshiftFunktionen in inversen Mizellen mit $\mu=1,2,4,8,16$ und 32, sowie in reinem Wasser. 

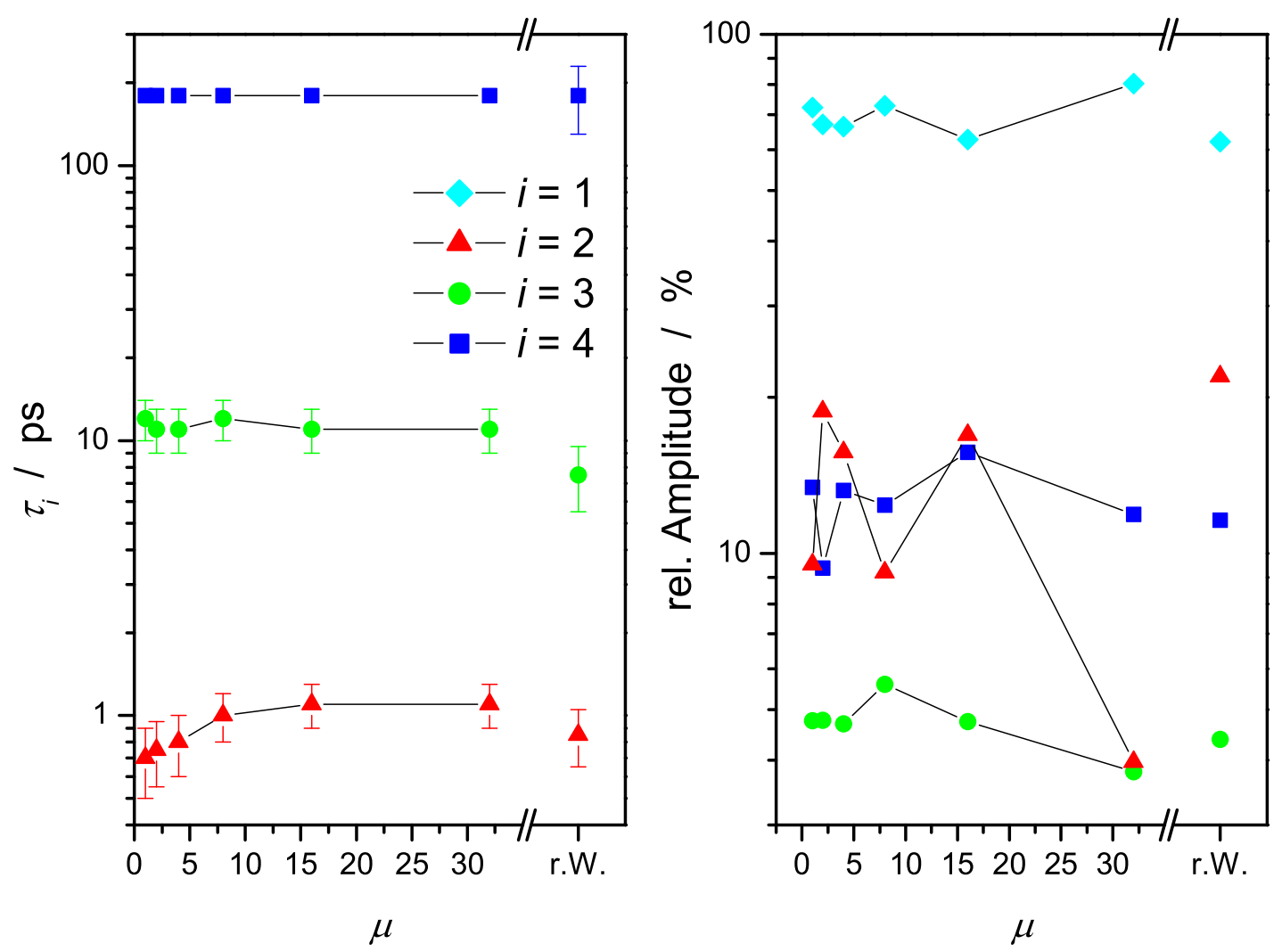

Abbildung 6.7: Graphische Darstellung der Anpassungsparameter der im PhotonEcho-Experiment erhaltenen Peakshift-Funktionen nach Gleichung 6.4 für inverse Mizellen in Abhängigkeit von $\mu$ und reines Wasser (r.W.).

\section{Zeitkonstante $\tau_{1}$}

Die relative Amplitude des Inertialteils in inversen Mizellen beträgt $70 \pm 10 \%$ und befindet sich damit in der Größenordnung des reinen Wassers. Die Zeitkonstanten zeigen im Rahmen der Meßgenauigkeit keinen Trend mit fortschreitender Einschränkung (nicht abgebildet). Auch wenn der Inertialteil der Peakshift-Funktion aufgrund der intrinsischen Eigenschaften des zeitintegrierten Photon-Echos nicht direkt mit der Solvatationskorrelationsfunktion in Verbindung gebracht werden kann, so ist diese Beobachtung dennoch in quantitativer Übereinstimmung mit molekulardynamischen Simulationen von Faeder et al. am AOT/Wasser-System [131]. In diesen Arbeiten wurde die Solvatation des Chromophors $\mathrm{I}_{2}^{-}$in inversen Mizellen simuliert. Dabei ergab sich für alle untersuchten Mizellengrößen, daß der Inertialteil ca. $70 \%$ der Solvatation ausmacht und keine Abhängigkeit vom Grad der Einschränkung aufweist. Dies wurde damit erklärt, daß der Chromophor sich innerhalb der freien Wasserphase der inversen Mizellen befindet und nicht an 
der Grenzschicht lokalisiert ist. Allerdings muß berücksichtigt werden, daß bei den von Faeder et al. untersuchten Mizellengrößen zwischen $60 \%$ und $95 \%$ des Wassers gebunden ist, so daß der Chromophor in jedem Falle eine Einschränkung erfährt und nicht von einer wirklich freien Wasserumgebung gesprochen werden kann. Obgleich der in der vorliegenden Arbeit verwendete Chromophor vermutlich direkt an der Grenzschicht lokalisiert ist, läßt die Unabhängigkeit des Inertialteils vom Grad der Einschränkung darauf schließen, daß die Moden des Wassers welche für den Inertialteil verantwortlich sind durch die heterogene Umgebung nicht oder nur unwesentlich beeinflußt werden. Von einer weiterführenden Diskussion wird aufgrund der schwachen Aussagekraft des zeitintegrierten Photon-Echo-Experimentes bezüglich des Inertialteils an dieser Stelle abgesehen.

\section{Zeitkonstante $\tau_{2}$}

Die Zeitkomponente um 1 ps, die für die intermolekularen gehinderten Translationsmoden des reinen Wassers charakteristisch ist, wird ebenso für alle inversen Mizellen wiedergefunden und unterscheidet sich nicht fundamental von der des reinen Wassers. Sie wird allerdings mit kleiner werdender Mizellengröße von $\tau_{2}=1.1$ ps bei $\mu=32$ bis $\tau_{2}=0.7$ ps bei $\mu=1$ etwas schneller. Dieser Trend ist überraschend, da mit zunehmender Einschränkung der Umgebung eher eine Verlangsamung der beobachteten Zeitkonstanten erwartet würde, wie andere Arbeiten zur Solvatationsdynamik in inversen Mizellen vermuten lassen (siehe Kapitel 3.3.2).

Levinger und Mitarbeiter fanden in Fluoreszenzaufkonvertierungsexperimenten am gleichen System mit der Farbstoffsonde Coumarin 343 eine Solvatationskorrelationsfunktion, die auf ultraschnellen Zeitskalen biexponentiell angepaßt wurde [105] (siehe auch Kapitel 3.3.2). Sie fanden eine schnelle Zeitkonstante um 200 fs, die keine Abhängigkeit von der Mizellengröße aufweist und der Dynamik des freien Wassers in Mizellen zugeschrieben wurde. Eine Komponente um 3 ps erscheint mit zunehmender Einschränkung immer langsamer und wurde dem gebundenen Wasser zugeordnet. Der amplitudengewichtete Mittelwert $\langle\tau\rangle$ dieser beiden Zeitkonstanten liegt um 1 ps und ist mit den hier ermittelten Zeitkonstanten vergleichbar (siehe Tabelle 3.3). Im Gegensatz zu den Einzelzeiten zeigt $\langle\tau\rangle$ keinen eindeutigen Trend mit wachsender Einschränkung. Bei der Bestimmung der Solvatationskorrelationsfunktion fand in dieser Arbeit keine Zeitnullanalyse nach Fee et al. [26] statt, d. h. nicht auflösbaren Zeitkomponenten wurde keinerlei Rechnung getragen. Eine Neuauswertung der Daten aus [105] mit Zeitnullanalyse zeigt interessanterweise analog den hier vorgestellten Beobachtungen einen beschleunigenden Effekt mit wachsender Einschränkung der Umgebung [102]. 
Eine vergleichbare Untersuchung der Solvatation von Wasser an einer Grenzschicht von Zirkonium-Nanopartikeln mit Hilfe der Farbstoffsonde Coumarin 343 von Levinger und Mitarbeitern weist einen ähnlichen Trend auf [263]. Die dort mit Hilfe der Fluoreszenzkonversionsspektroskopie ermittelten Solvatationskorrelationsfunktionen weisen eine deutliche Beschleunigung der Dynamik an der Grenzschicht der Nanopartikel im Vergleich zur reinen Wasserphase auf, was mit einem Aufbrechen des Wasserstoffbrückennetzwerkes an der Grenzschicht erklärt wird. Durch dieses Aufbrechen wird es den Wassermolekülen an der Grenzschicht ermöglicht sich freier als in reinem Wasser zu bewegen, was in einer beschleunigten Solvatation resultiert.

Für die 1 ps Zeitkomponente bietet sich ein Vergleich mit den molekulardynamischen Simulationen von Faeder et al. an [131]. Dort zeigt sich im Gegensatz zu dem hier beobachteten Trend, daß die Solvatation durch zunehmende Einschränkung der Umgebung in diesem Zeitbereich tatsächlich verlangsamt wird. Der dort beobachtete Effekt auf die Solvatationskorrelationsfunktion im beobachteten Zeitfenster bis $2 \mathrm{ps}$ ist allerdings überraschend klein. Es ist fraglich, ob der dort beobachtete Effekt überhaupt mit experimentellen Methoden nachgewiesen werden könnte. Im Rahmen der molekulardynamischen Simulationen von Faeder et al. läßt sich daher zumindest die geringe Abhängigkeit der Solvatationsdynamik auf ultraschnellen Zeitskalen verstehen, auch wenn der Trend nicht wiedergegeben wird.

Es ist zu beachten, daß der Grenzwert der Zeitkonstanten zu großen Mizellen hin nicht der Zeitkonstanten des reinen Wassers von $\tau_{2}=0.85$ ps entspricht. Diese Diskrepanz könnte wie bei den stationären Spektren durch die ionischen Verhältnisse im Wassereinschluß der inversen Mizellen erklärt werden. Der Effekt ist allerdings sehr gering und im Bereich der Fehlerbalken (siehe Abbildung 6.7). Ähnlich starke Schwankungen der relativen Amplitude in Abhängigkeit von $\mu$, die keinen Trend erkennen lassen, wurden auch von Levinger und Mitarbeitern beobachtet [105].

Festzuhalten bleibt, daß in den inversen Mizellen intermolekulare gehinderte Translationsmoden des Wassers um 1 ps weiterhin eine Rolle spielen. Der Grund für die leichte Beschleunigung der Solvatation durch diesen Freiheitsgrad ist vermutlich auf ein modifiziertes Wasserstoffbrückennetzwerk zurückzuführen, was in einer größeren Bewegungsfreiheit der Wassermoleküle an der Grenzschicht resultiert.

\section{Zeitkonstante $\tau_{3}$}

Die für die Rotationsdiffusion einzelner Moleküle charakteristische Zeitkonstante um 10 ps wird in allen inversen Mizellen wiedergefunden und erweist sich als 
weitestgehend unabhängig von $\mu$. Dies läßt vermuten, daß der Freiheitsgrad der Solvatation durch diffusive Rotation einzelner Moleküle durch eine zunehmende Einschränkung der Umgebung nicht wesentlich beeinflußt wird. Beim Einbringen des gleichen Chromophors an eine Lipidmembran/Wasser-Grenzschicht wurde im Einklang mit dieser Beobachtung auch kein großer Effekt bezüglich dieser Zeitkomponenten gefunden [50,79]. Die Zeitkonstante erscheint hier allerdings mit $11 \pm 2$ ps im Vergleich zur Komponente in reinem Wasser etwas verlangsamt, was vermutlich durch die veränderte Wasserstruktur durch ionische Kopfgruppen und Gegenionen in inversen Mizellen im Vergleich zu reinem Wasser erklärt werden kann. Die relative Amplitude dieses Freiheitsgrades ist sehr gering und schwankt zwischen $3.8 \%$ und $5.6 \%$ und ist damit mit der Amplitude in reinem Wasser vergleichbar. Vermutlich hat in inversen Mizellen die Rotationsdiffusion einzelner Wassermoleküle einen ähnlich großen Anteil an der Solvatation wie in der reinen Wasserphase.

\section{Zeitkonstante $\tau_{4}$}

Die langsamste Komponente, die auf 180 ps festgesetzt wurde, ist durch einen Vergleich mit reinem Wasser vermutlich auf die kollektive Rotationsdiffusion des Lösungsmittels und/oder des Chromophors zurückzuführen und zeigt eine vergleichsweise konstante Amplitude um $12 \%$. Eine vergleichbare Zeitkonstante zwischen 200 und 400 ps wurde auch von Levinger und Mitarbeitern im Fluoreszenzaufkonvertierungsexperiment gefunden [105]. Sie wird nach diesen Untersuchungen mit zunehmender Mizellengröße immer schneller, erreicht aber nie den Wert des reinen Wassers. Die Zeitkomponente wurde dort ebenso den kollektiven Bewegungen des Wassers an der Grenzschicht zugeordnet. Weiterreichende Aussagen bezüglich dieser Komponente sind an dieser Stelle aufgrund der genannten Limitierungen des Experimentes nicht möglich.

\subsection{Schwingungsdynamik}

Um die Schwingungsstruktur des verwendeten Chromophors HIDCI zu studieren, wurden Transient-Grating-Messungen durchgeführt. Durch impulsive Präparation kohärenter Überlagerungen benachbarter Schwingungseigenzustände, deren Energiedifferenzen durch die spektrale Bandbreite des Anregungspulses erfaßt werden, enthält das TG-Signal sowohl Wellenpaketdynamik im elektronischen Grundzustand als auch Dynamik des elektronisch angeregten Zustandes. Die Wellenpaketdynamik des Grundzustandes ist auf resonante, impulsivstimulierte Raman-Streuung zurückzuführen [264]. Dadurch sind diese Messungen 
zu Raman-Streuexperimenten in der Frequenzdomäne komplementär, welche lediglich Grundzustandsdynamik beinhalten.

Die erhaltenen Transient-Grating-Signale sowohl für reines Wasser als auch für die inversen Mizellen unterschiedlicher Größe sind in Abbildung 6.8 dargestellt. Alle Signale weisen eine sehr ähnliche Dynamik auf. Kleinere Unterschiede resultieren vorrangig aus dem nicht-oszillatorischen Hintergrund. Das exponentielle Abklingen erfolgt beispielsweise für die Messung des reinen Wassers deutlich schneller als in den Mizellen.

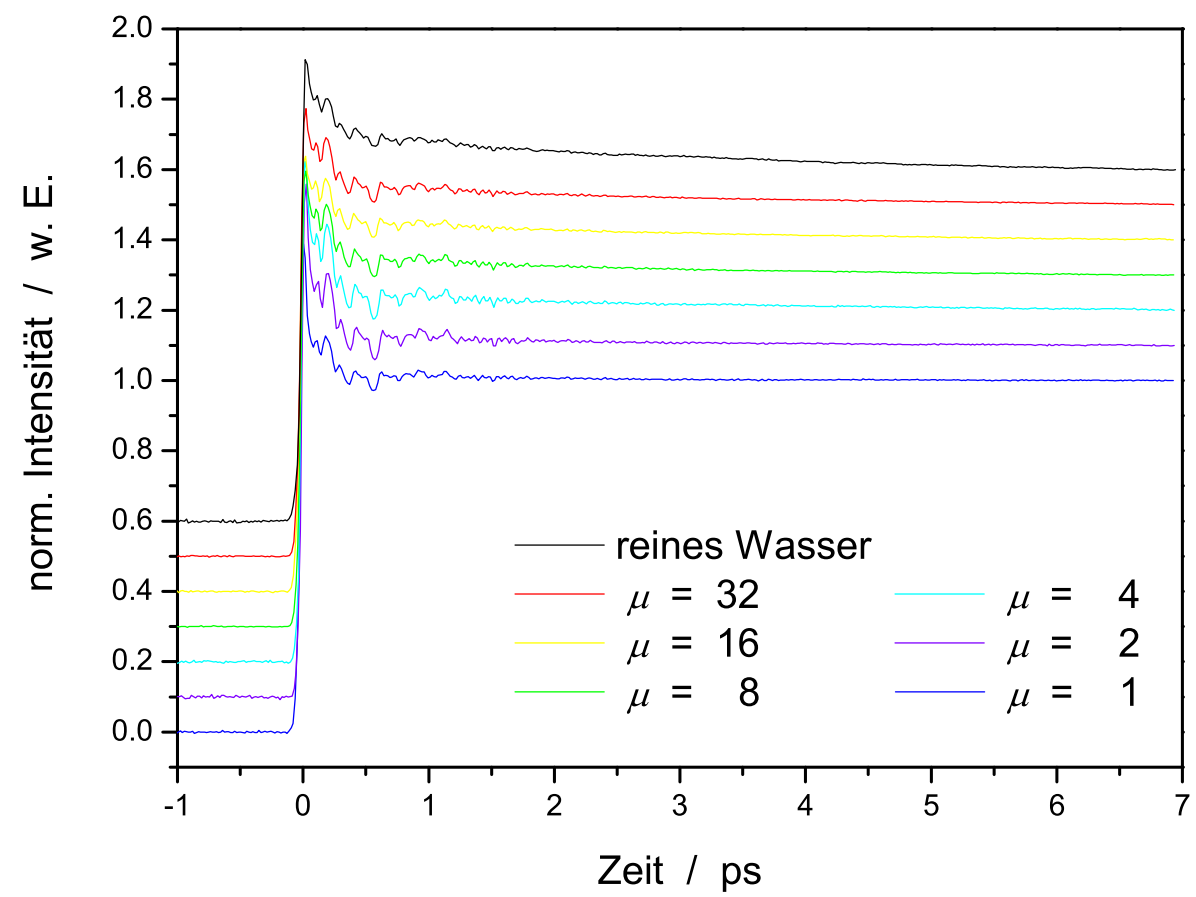

Abbildung 6.8: Transient-Grating-Messungen für HIDCI in reinem Wasser und inversen Mizellen unterschiedlicher Größe. Zur besseren Veranschaulichung sind die einzelnen Messungen bezüglich der Ordinate relativ zueinander verschoben.

Zur näheren Untersuchung der Schwingungsstruktur wurden die Daten einer sogenannten LPSVD-Analyse unterzogen (LPSVD, Linear Prediction Singular Value Decomposition). Diese Methode simuliert das Signal als eine Summe aus exponentiell gedämpften Kosinusfunktionen gemäß [265]:

$$
I(t)=\sum_{i} A_{i} \cos \left(\omega_{i} t+\phi_{i}\right) \exp \left(-\frac{t}{\tau_{i}}\right) .
$$

Dabei bezeichnet $A_{i}$ die Amplitude einer Schwingung $i$ mit der Frequenz $\omega_{i}$ und der Phase $\phi_{i}$. Die Dämpfung der entsprechenden Schwingung wird mit $\tau_{i}$ beschrieben. Die Methode ermöglicht eine einfache Entfernung nicht-oszillatorischer 
Beiträge durch Festlegung einer unteren Grenze für die Frequenz $\omega_{i}$, ab der Schwingungen als oszillatorisch bezeichnet werden. Sie wurde hier $\mathrm{zu} 80 \mathrm{~cm}^{-1}$ festgelegt. Niederfrequentere Beiträge wurden als nicht-oszillatorisch behandelt und von den TG-Signalen subtrahiert. An den verbliebenen Residuen wurde eine Frequenzanalyse nach der FFT-Methode (FFT, Fast Fourier Transformation) durchgeführt, um so Leistungsspektren (Power Spectra) zu erhalten.

Um die ermittelten Schwingungsfrequenzen zuordnen zu können, wird in Abbildung 6.9 zunächst das Leistungsspektrum von HIDCI in reinem Wasser einem post-resonanten Ramanspektrum von HIDCI in Ethanol gegenübergestellt [50].

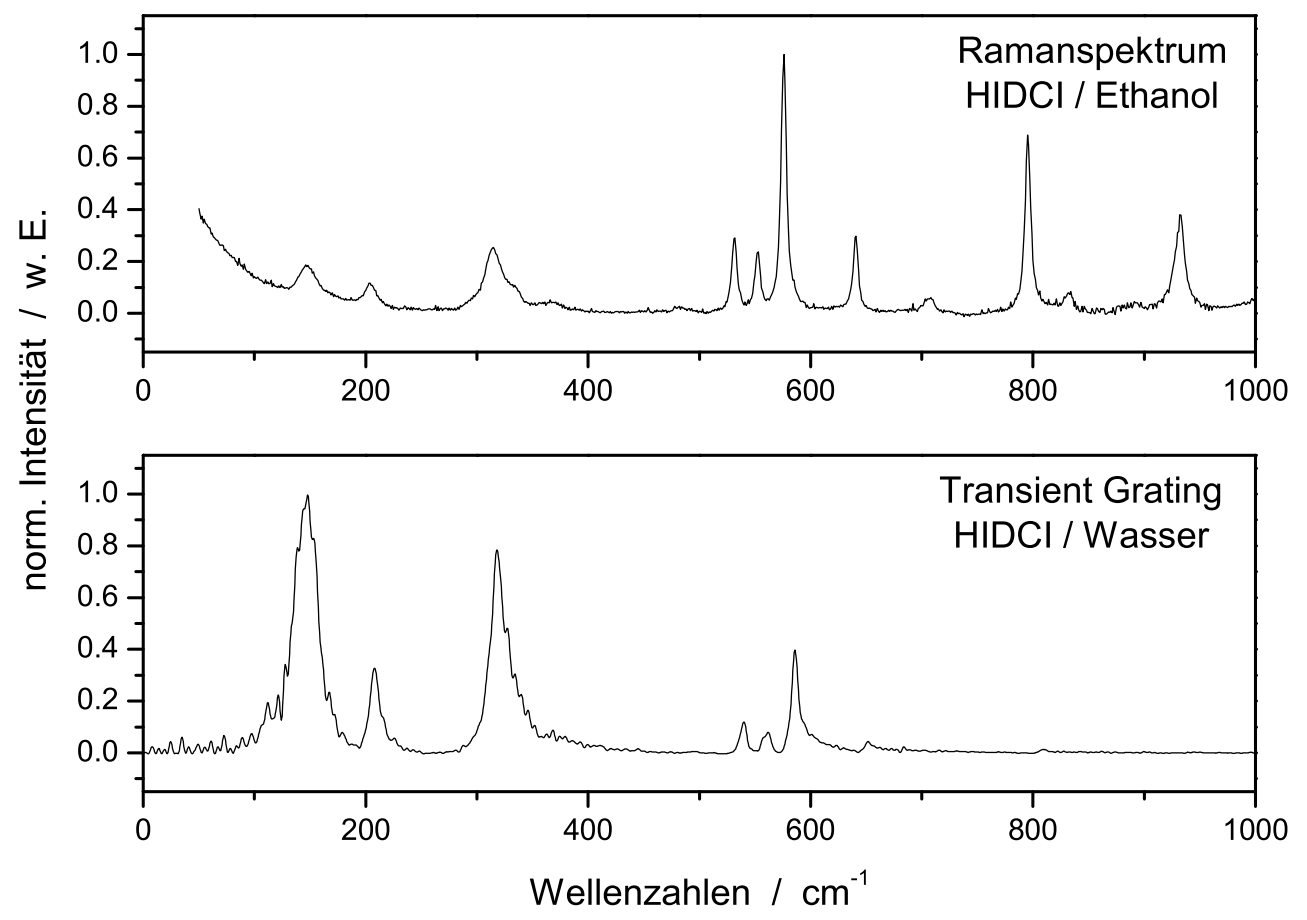

Abbildung 6.9: Gegenüberstellung des post-resonanten Ramanspektrums von HIDCI in Ethanol und des Leistungsspektrums der TG-Messung von HIDCI in Wasser nach Entfernung nicht-oszillatorischer Beiträge.

Die beiden Spektren weisen eine gute qualitative Übereinstimmung auf, wenn auch die Banden von HIDCI in Wasser im Gegensatz zu den Banden im Ramanspektrum allesamt um $5-15 \mathrm{~cm}^{-1}$ zu höheren Wellenzahlen verschoben sind. Unterschiede in den Bandenintensitäten sind vorrangig auf die endliche Bandbreite der Anregungslaserpulse zurückzuführen, was bei der Analyse der TG-Signale nicht berücksichtigt wurde, d. h. es fand keine Entfaltung von der Geräteantwortfunktion statt. Insbesondere bei niedrigen Frequenzen lassen sich Unterschiede der Bandenintensitäten zudem durch die unterschiedlichen Resonanzbedingungen 
beider Experimente erklären. Die spektralen Lagen der Banden stimmen insgesamt dennoch sehr gut überein, was darauf hindeutet, daß die Schwingungsstruktur des Chromophors in Grund- und angeregtem Zustand sehr ähnlich sein muß. So taucht im TG-Spektrum keine Bande auf, die exklusiv dem angeregten Zustand des Chromophors zugeordnet werden könnte. Desweiteren sind die Spektren von HIDCI in Lösung in qualitativer Übereinstimmung mit Raman-Spektren des gleichen Chromophors in KBr-Proben mit Ausnahme einer zusätzlichen Bande ca. $18 \mathrm{~cm}^{-1}$ unterhalb der niederfrequenten Komponente der Triplett-Struktur in Flüssigkeiten um $550 \mathrm{~cm}^{-1}$. Eine Ursache für diesen Unterschied ist bislang nicht bekannt, da zudem die Zuordnung der Banden bisher noch recht lückenhaft ist $[266,267,268]$. Nach Sato et al. ist die stärkste Bande der Triplett-Struktur bei $576 \mathrm{~cm}^{-1}$ einer Deformationsmode der Polymethin-Kette zuzuordnen, während die niederfrequente Bande des Tripletts bei $532 \mathrm{~cm}^{-1}$ aus einer Out-of-PlaneBiegeschwingung der $\mathrm{C}-\mathrm{C}\left(\mathrm{CH}_{3}\right)_{2}$-C-Gruppe des Indolfragments im Chromophor resultiert [266].

Das Leistungsspektrum des reinen Wassers ist in Abbildung 6.10 schließlich den Spektren des Chromophors in inversen Mizellen gegenübergestellt.

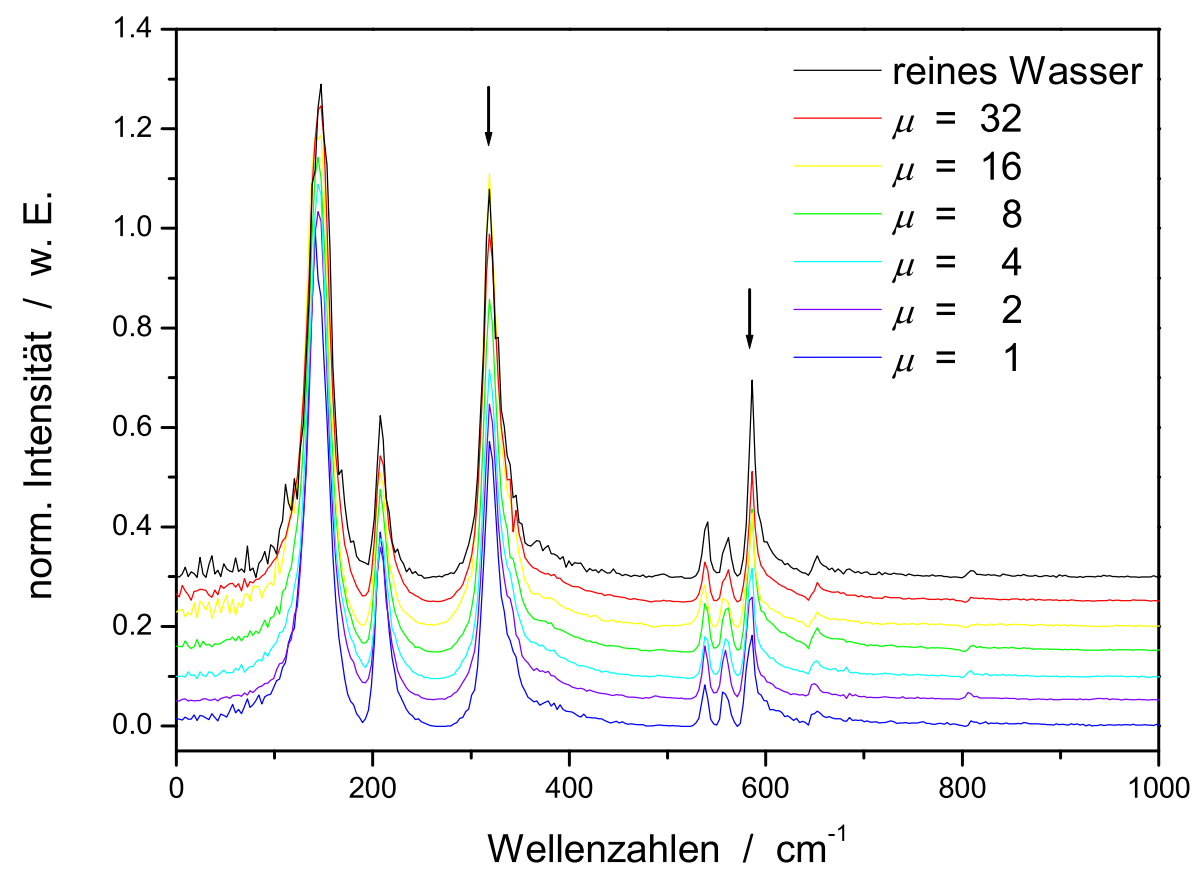

Abbildung 6.10: Leistungsspektren der TG-Messungen von HIDCI in reinem Wasser und in inversen Mizellen unterschiedlicher Größe nach Entfernung nichtoszillatorischer Beiträge. Zur besseren Veranschaulichung sind die einzelnen Messungen bezüglich der Ordinate relativ zueinander verschoben. 
Die erhaltenen Spektren wurden auf die intensivste Bande bei $144 \mathrm{~cm}^{-1}$ normiert und weisen untereinander größte Ähnlichkeit auf. Die Intensitäten der einzelnen Banden stimmen in erster Näherung überein. Offensichtlich wird die Schwingungsstruktur des Chromophors durch Einbringen des Farbstoffes in das Wassernanotröpfchen inverser Mizellen nur unwesentlich beeinflußt. Bei näherer Betrachtung der Bandenintensitäten fällt jedoch auf, daß die Banden bei $319 \mathrm{~cm}^{-1}$ und $586 \mathrm{~cm}^{-1}$ mit kleiner werdender Mizellengröße und damit wachsender Einschränkung der Umgebung in ihrer Intensität abnehmen. Die Intensitäten der übrigen Banden bleiben im Rahmen der Meßgenauigkeit konstant. Eine weiterreichende Interpretation der hier beobachteten Effekte ist an dieser Stelle allerdings nicht möglich.

\subsection{Resümee - Inverse Mizellen}

Zusammenfassend läßt sich feststellen, daß die Solvatationsdynamik des eingeschränkten Wassers in inversen Mizellen auf ultraschnellen Zeitskalen der Solvatationsdynamik in reinem Wasser unter Verwendung der identischen Farbstoffsonde überraschend ähnlich ist. Es wird keine dramatische Abhängigkeit der Solvatation vom jeweiligen Grad der Einschränkung in inversen Mizellen beobachtet, was mit molekulardynamischen Simulationen von Faeder et al. im Einklang steht. In den Wassernanotröpfchen inverser Mizellen wird analog den Untersuchungen an reinem Wasser ein stark ausgeprägter Inertialteil gefunden, welcher wie in den MD-Simulationen vorhergesagt, im Rahmen der Meßgenauigkeit unabhängig vom Grad der Einschränkung ist. Darüberhinaus wird die den intermolekularen gehinderten Translationsmoden zuzuordnende Zeitkonstante um 1 ps gefunden. Sie weist den überraschenden Trend einer beschleunigten Dynamik mit zunehmender Einschränkung der Umgebung auf. Die Ursache für dieses Verhalten ist vermutlich auf das Aufbrechen des Wasserstoffbrückennetzwerkes an der Grenzschicht zurückzuführen. Ähnliche Effekte wurden in Untersuchungen von Levinger mit Fluoreszenzaufkonvertierungsexperimenten an vergleichbaren Systemen beobachtet. Auch die Zeitkomponente der Rotationsdiffusion einzelner Wassermoleküle um 10 ps wird in sämtlichen Messungen beobachtet und erweist sich als weitestgehend unabhängig vom Grad der Einschränkung. Im Vergleich zur Messung an reinem Wasser erscheint diese Zeitkonstante allerdings etwas verlangsamt. Darüberhinaus werden für alle untersuchten Systeme auch noch langsamere Zeitkomponenten im Bereich einiger hundert Pikosekunden gefunden, die aber aufgrund der Restriktionen des 3PEPS-Experimentes in der Boxcar-Geometrie nicht weiter diskutiert wurden. Darüberhinaus zeigt sich, daß die Schwingungsdynamik der eingesetzten Farbstoffsonde HIDCI mit zunehmender Einschränkung der Umgebung leicht gestört wird. 
Insgesamt gesehen stellen die hier vorgestellten Ergebnisse nach bestem Kenntnisstand die ersten ultraschnellen Untersuchungen zur Solvatationsdynamik von Wasser in eingeschränkten Umgebungen am Modellsystem der inversen Mizellen mit Hilfe der 3-Puls Photo-Echo Technik dar. Die ersten überraschenden Beobachtungen müssen durch weiterführende Untersuchungen bestätigt und verfeinert werden. Der Vergleich mit molekulardynamischen Simulationen erweist sich dabei als äußerst fruchtbar, um die stattfindenden Prozesse der Solvatation in eingeschränkten Umgebungen auf molekularer Ebene im Detail zu verstehen. 


\section{Kapitel 7}

\section{Ausblick}

In dieser Arbeit wurden neueste Erkenntnisse zur Solvatationsdynamik an ausgewählten biologischen Grenzschichten vorgestellt. So wurde die Dynamik der Solvatation an Lipidmembran/Wasser-Grenzschichten über einen außerordentlich großen dynamischen Bereich von über fünf Größenordnungen charakterisiert, wobei erstmals die Technik der Fluoreszenzaufkonvertierung an solchen Systemen zum Einsatz kam. Die ultraschnelle Solvatation in inversen Mizellen wurde hier erstmals mit Photon-Echo Techniken untersucht. Die durchgeführten Experimente erwiesen sich im Hinblick auf die Fragestellung der Arbeit als äußerst ergiebig und ermöglichten einen tieferen Einblick in die elementaren Vorgänge an biologischen Grenzschichten. Dennoch stellt das Forschungsgebiet der ultraschnellen Dynamiken in heterogenen Systemen noch immer ein Feld größter wissenschaftlicher Herausforderungen sowohl in experimenteller als auch in theoretischer Hinsicht dar. Daher soll in diesem Kapitel ein kurzer Ausblick auf experimentelle Verbesserungen und zukünftige Forschungsschwerpunkte gegeben werden.

\section{Lipid-Vesikel}

Die Untersuchungen der Solvatation an Lipid-Vesikeln haben gezeigt, daß in Membranumgebungen Dynamiken auf ultraschnellen Zeitskalen in einem erheblichen Maße an der Stabilisierung eines erzeugten Nichtgleichgewichtszustandes teilhaben. Um diese genauer untersuchen zu können, ist es unumgänglich die derzeit erreichbare Zeitauflösung der Fluoreszenzkonversionsapparatur von ca. 0.5 ps signifikant zu verbessern. Das untere Limit eines Fluoreszenzkonversionsexperimentes liegt bei etwa 100 fs, weshalb zur Erfassung noch schnellerer Komponenten hochauflösende Techniken, wie z. B. das 3-Puls Photon-Echo Peakshift Experiment (3PEPS) herangezogen werden müssen. Zeitintegrierte 3PEPS-Studien wurden bereits von Helge Bürsing erfolgreich an vergleichbaren Systemen durch- 
geführt. Jedoch ergeben sich auch hier Limitierungen bezüglich der Sensitivität dieses Experimentes auf den Inertialteil der Solvatation, wie in Kapitel 2.3 dargestellt wurde. Da die Untersuchung von Lipid-Vesikeln jedoch üblicherweise in Küvetten durchgeführt wird, birgt die Durchführung eines zeitaufgelösten PhotonEcho-Experimentes einige experimentelle Schwierigkeiten. Die Verbesserung der experimentellen Techniken stellt dementsprechend eine technische Herausforderung für zukünftige ultraschnelle Experimente an biologischen Systemen dar.

Die hier durchgeführten Experimente haben gezeigt, daß die gewählte Farbstoffsonde Laurdan einige photophysikalische Besonderheiten aufweist. Während es einerseits von Interesse ist, die Photophysik des Laurdans im Detail zu verstehen, verkomplizieren sie auf der anderen Seite die Untersuchung der reinen Solvatationsdynamik in vesikulären Systemen. Daher sind Experimente mit einer Farbstoffsonde denkbar, bei der auftretende Charge-Transfer-Komplexe durch eine Verbrückung der Dimethylaminogruppe effektiv unterbunden werden.

Um die in dieser Arbeit vorgestellte Interpretation der in Lipid-Vesikeln stattfindenden Solvatationsprozesse zu verifizieren, ist es weiterhin vonnöten die ultraschnelle Dynamik auch unterhalb des Hauptphasenübergangs eingehend zu untersuchen. Ebenso ist es von Interesse, die Solvatation an verschiedenen Orten entlang der Membrannormalen zu charakterisieren, ausgehend von der Kettenregion bis in die freie Wasserphase. Dazu müßten geeignete Farbstoffsonden mit wohldefinierter Lokalisierung ihrer Chromophore herangezogen werden. Um den tatsächlichen Verhältnissen in der Natur näher zu kommen, bieten sich darüberhinaus Experimente an biologischen Membranen an, die beispielsweise Cholesterin oder Proteine in ihrer Grenzschicht inkorporiert haben.

Um die experimentellen Ergebnisse in solchen biologischen Systemen besser verstehen zu können, bieten sich Vergleiche mit molekulardynamischen Simulationen an. Dabei steht die Simulation dynamischer Vorgänge an Membran/WasserGrenzschichten, die direkt mit den experimentellen Ergebnissen vergleichbar wären, noch aus. Von dieser Seite bietet sich ein erhebliches Forschungspotential.

\section{Inverse Mizellen}

Das 3-Puls Photon-Echo Peakshift Experiment hat sich als ein nützliches Werkzeug bei der Untersuchung der ultraschnellen Solvatationsdynamik in inversen Mizellen erwiesen. Die Durchführung der Experimente in der Boxcar-Geometrie führte allerdings dazu, daß keinerlei Aussagen bezüglich der auftretenden, langsamen Zeitkonstanten getroffen werden konnten. Um diese Komponenten zu untersuchen, bieten sich Fluoreszenzkonversions- und zeitkorrelierte Einzelphotonenzählexperimente an, wie sie bei den Lipid-Vesikeln zum Einsatz kamen. 
Die in inversen Mizellen durchgeführten Studien machen deutlich, daß es notwendig ist, die genaue Lage der Farbstoffsonde im Wassereinschluß der Mizellen zu kennen, um die erhaltenen Erkenntnisse richtig einordnen zu können. Es bietet sich daher an, auch in diesem System die Farbstoffsonde über eine nicht-kovalente Verankerung an der Grenzschicht zu lokalisieren. Eine weitere Schwierigkeit bei der Interpretation der Daten ergibt sich aus den ionischen Verhältnissen innerhalb der untersuchten AOT-Mizellen. Es ist daher naheliegend die Solvatation auch in neutralen Systemen zu untersuchen, um den Einfluß durch in der Lösung befindliche Ionen ausschließen zu können.

Die Untersuchungen an inversen Mizellen haben darüberhinaus gezeigt, daß sich das Zusammenspiel zwischen molekulardynamischen Simulationen und experimentellen Untersuchungen bei der Interpretation ultraschneller Prozesse in eingeschränkten Umgebungen als äußerst fruchtbar erweist. Eine Weiterführung molekulardynamischer Simulationen ist daher für ein tieferes Verständnis der zugrundeliegenden Dynamiken in biologischen Systemen unumgänglich.

\section{Schlußbemerkung}

Die Dynamik der Solvatation in mikroheterogenen Umgebungen wurde in dieser Arbeit lediglich an zwei repräsentativen, biologischen Modellsystemen untersucht. Darüberhinaus sind mit den hier vorgestellten Techniken eine Fülle analoger Experimente an weiteren Systemen denkbar. So bieten sich beispielsweise Studien an Cyclodextrinen, Zeoliten, Proteinen oder auch der DNA an. Hier eröffnet sich ein riesiges Forschungsfeld, welches noch am Anfang steht und daher eingehender Untersuchung bedarf. Die Studien versprechen einen interessanten Einblick in die Elementarprozesse bei der Solvatation in eingeschränkten, biologischen Umgebungen. 



\section{Literaturverzeichnis}

[1] A. H. Zewail, Femtochemie: Studium der Dynamik der chemischen Bindung auf atomarer Skala mit Hilfe ultrakurzer Laserpulse (Nobel-Aufsatz), Angew. Chem., 112 (2000) 26882738 .

[2] E. W. Castner Jr., M. Maroncelli, Solvent Dynamics Derived from Optical Kerr Effect, Dielectric Dispersion, and Time-Resolved Stokes Shift Measurements: An Empirical Comparison, J. Mol. Liq., 77 (1998) 1-36.

[3] B. Bagchi, R. Biswas, Polar and Nonpolar Solvation Dynamics, Ion Diffusion, and Vibrational Relaxation: Role of Biphasic Solvent Response in Chemical Dynamics, In: I. Prigogine, S. A. Rice (Hrsg.), Adv. Chem. Phys., Bd. 109 (John Wiley \& Sons, New York, 1999), 207-433.

[4] G. Cevc, D. Marsh, Phospholipid Bilayers: Physical Principles and Models (John Wiley \& Sons, New York, 1987).

[5] E. Grell (Hrsg.), Membrane Spectroscopy, Bd. 31 von Molecular Biology, Biochemistry and Biophysics (Springer, Berlin, 1981).

[6] M. Shinitzky (Hrsg.), Biomembranes: Vol. 1 Physical Aspects (VCH, Weinheim, 1993).

[7] M. P. Pileni (Hrsg.), Structure and Reactivity in Reverse Micelles, Bd. 65 von Studies in Physical and Theoretical Chemistry (Elsevier Science Publishers B. V., Amsterdam, 1989).

[8] P. L. Luisi, B. E. Straub (Hrsg.), Reverse Micelles: Biological and Technological Relevance of Amphiphilic Structures in Apolar Media (Plenum Press, New York, 1984).

[9] J. M. Berg, J. L. Tymoczko, L. Stryer, Biochemie (Spektrum Akademischer Verlag, Heidelberg, 2003), 5. Aufl.

[10] B. Bagchi, E. W. Castner, G. R. Fleming, On the Generalized Continuum Model of Dipolar Solvation Dynamics, J. Mol. Struct., 194 (1989) 171-181.

[11] B. Bagchi, Dynamics of Solvation and Charge Transfer Reactions in Dipolar Liquids, Annu. Rev. Phys. Chem., 40 (1989) 115-141.

[12] P. F. Barbara, W. Jarzȩba, Ultrafast Photochemical Intramolecular Charge and Excited State Solvation, In: D. H. Volman, G. S. Hammond, K. Gollnick (Hrsg.), Adv. Photochem., Bd. 15 (John Wiley \& Sons, New York, 1990), 1-67.

[13] B. Bagchi, D. W. Oxtoby, G. R. Fleming, Theory of the Time Development of the Stokes Shift in Polar Media, Chem. Phys., 86 (1984) 257-267.

[14] M. Berg, Comparison of a Viscoelastic Theory of Solvation Dynamics to Time-Resolved Experiments in a Nonpolar Solution, Chem. Phys. Lett., 228 (1994) 317-322. 
[15] M. Berg, Viscoelastic Continuum Model of Nonpolar Solvation. 1. Implications for Multiple Time Scales in Liquid Dynamics, J. Phys. Chem. A, 102 (1998) 17-30.

[16] D. S. Larsen, Investigation of Non-Dipolar Solvation Dynamics, Dissertation, University of Chicago (2001).

[17] H. Fröhlich, Theory of Dielectrics: Dielectric Constant and Dielectric Loss, Monographs on the Physics and Chemistry of Materials (Clarendon Press, Oxford, 1968), 2. Aufl.

[18] S. Mukamel, Principles of Nonlinear Spectroscopy, Bd. 6 von Oxford Series in Optical and Imaging Sciences (Oxford University Press, New York, 1995).

[19] W. R. Ware, S. K. Lee, G. J. Brant, P. P. Chow, Nanosecond Time-Resolved Emission Spectroscopy: Spectral Shifts due to Solvent-Excited Solute Relaxation, J. Chem. Phys., 54 (1971) 4729-4737.

[20] S. K. Chakrabarti, W. R. Ware, Nanosecond Time-Resolved Emission Spectroscopy of 1-Anilino-8-naphthalene Sulfonate, J. Phys. Chem., 55 (1971) 5494-5498.

[21] G. C. Walker, W. Jarzȩba, T. J. Kang, A. E. Johnson, P. F. Barbara, Ultraviolet Femtosecond Fluorescence Spectroscopy: Techniques and Applications, J. Opt. Soc. Am. B, 7 (1990) 1521-1527.

[22] M. Cho, S. J. Rosenthal, N. F. Scherer, L. D. Ziegler, G. R. Fleming, Ultrafast Solvent Dynamics: Connection between Time Resolved Fluorescence and Optical Kerr Measurements, J. Chem. Phys., 96 (1992) 5033-5038.

[23] G. R. Fleming, M. Cho, Chromophore-Solvent Dynamics, Annu. Rev. Phys. Chem., 47 (1996) 109-134.

[24] R. Jimenez, G. R. Fleming, P. V. Kumar, M. Maroncelli, Femtosecond Solvation Dynamics of Water, Nature, 369 (1994) 471-473.

[25] G. R. Fleming, S. A. Passino, Y. Nagasawa, The Interactions of Solutes with their Environment, Philos. Trans. R. Soc. London, Ser. A, 356 (1998) 389-404.

[26] R. S. Fee, M. Maroncelli, Estimating the Time-Zero Spectrum in Time-Resolved Emission Measurements of Solvation Dynamics, Chem. Phys., 183 (1994) 235-247.

[27] H. Bürsing, Photon-Echo-Spektroskopie zur Dynamik der Solvatation in Wasser und an Lipidmembran-Wasser-Grenzschichten, Dissertation, Universität Göttingen (2002).

[28] M. S. Pshenichnikov, K. Duppen, D. A. Wiersma, Time-Resolved Photon Echo Probes Bimodal Solvent Dynamics, Phys. Rev. Lett., 74 (1995) 674-677.

[29] W. P. de Boeij, M. S. Pshenichnikov, D. A. Wiersma, System-Bath Correlation Function Probed by Conventional and Time-Gated Stimulated Photon Echo, J. Phys. Chem., 100 (1996) 11806-11823.

[30] W. P. de Boeij, M. S. Pshenichnikov, D. A. Wiersma, On the Relation between the EchoPeak Shift and Brownian-Oscillator Correlation Function, Chem. Phys. Lett., 253 (1996) $53-60$.

[31] W. P. de Boeij, M. S. Pshenichnikov, D. A. Wiersma, Ultrafast Solvation Dynamics Explored by Femtosecond Photon Echo Spectroscopies, Ann. Rev. Phys. Chem., 49 (1998) 99-123.

[32] M. Cho, J.-Y. Yu, T. Joo, Y. Nagasawa, S. A. Passino, G. R. Fleming, The Integrated Photon Echo and Solvation Dynamics, J. Phys. Chem., 100 (1996) 11944-11953. 
[33] S. D. Silvestri, A. M. Weiner, J. G. Fujimoto, E. P. Ippen, Femtosecond Dephasing Studies of Dye Molecules in a Polymer Host, Chem. Phys. Lett., 112 (1984) 195.

[34] A. M. Weiner, S. D. Silvestri, E. P. Ippen, Three-Pulse Scattering for Femtosecond Dephasing Studies: Theory and Experiment, J. Opt. Soc. Am. B, 2 (1985) 654-661.

[35] P. Vöhringer, D. C. Arnett, T.-S. Yang, N. F. Scherer, Time-Gated Photon Echo Spectroscopy in Liquids, Chem. Phys. Lett., 237 (1995) 387-398.

[36] W. P. de Boeij, Ultrafast Solvation Dynamics Explored by Nonlinear Optical Spectroscopy, Dissertation, Rijksuniversiteit Groningen (1997).

[37] P. N. Butcher, D. Cotter, The Elements of Nonlinear Optics, Bd. 9 von Cambridge Studies in Modern Optics (Cambridge University Press, Cambridge, 1990).

[38] M. J. Lang, X. J. Jordanides, X. Song, G. R. Fleming, Aqueous Solvation Dynamics Studied by Photon Echo Spectroscopy, J. Chem. Phys., 110 (1999) 5884-5892.

[39] L. Allen, J. H. Eberly, Optical Resonance and Two-Level Atoms (Dover Publications, New York, 1987).

[40] K. Duppen, D. A. Wiersma, Picosecond Multiple-Pulse Experiments involving Spatial and Frequency Gratings: A Unifying Nonperturbational Approach, J. Opt. Soc. Am. B, 3 (1986) 614-621.

[41] T. Joo, Y. Jia, J.-Y. Yu, M. J. Lang, G. R. Fleming, Third-Order Nonlinear Time Domain Probes of Solvation Dynamics, J. Chem. Phys., 104 (1996) 6089-6108.

[42] E. J. Brown, Q. Zhang, M. Dantus, Femtosecond Transient-Grating Techniques: Population and Coherence Dynamics involving Ground and Excited State, J. Chem. Phys., 110 (1999) 5772-5788.

[43] A. L. Lehninger, D. L. Nelson, M. M. Cox, Prinzipien der Biochemie (Spektrum Akademischer Verlag, Heidelberg, 1994), 2. Aufl.

[44] M. Maroncelli, The Dynamics of Solvation in Polar Liquids, J. Mol. Liq., 57 (1993) 1-37.

[45] K. Winkler, J. Lindner, H. Bürsing, P. Vöhringer, Ultrafast Raman-Induced Kerr-Effect of Water: Single Molecule versus Collective Motions, J. Chem. Phys., 113 (2000) 46744682 .

[46] B. Zolotov, A. Gan, B. D. Fainberg, D. Huppert, Resonance Heterodyne Optical Kerr Spectroscopy of Solvation Dynamics in Water and $D_{2} O$, Chem. Phys. Lett., 265 (1997) 418-426.

[47] Y. J. Chang, E. W. Castner Jr., Femtosecond Dynamics of Hydrogen-Bonding Solvents. Formamide and N-Methylformamide in Acetonitrile, DMF and Water, J. Chem. Phys., 99 (1993) 113-125.

[48] S. Palese, L. Schilling, R. J. D. Miller, P. R. Staver, W. T. Lotshaw, Femtosecond Optical Kerr Effect Studies of Water, J. Phys. Chem., 98 (1994) 6308-6316.

[49] W. Jarzȩba, G. C. Walker, A. E. Johnson, M. A. Kahlow, P. F. Barbara, Femtosecond Microscopic Solvation Dynamics of Aqueous Solutions, J. Phys. Chem., 92 (1988) 7039 7041.

[50] H. Bürsing, D. Ouw, S. Kundu, P. Vöhringer, Probing Solvation Dynamics in Liquid Water and at Phospholipid/Water Interfaces with Femtosecond Photon Echo Spectroscopies, Phys. Chem. Chem. Phys., 3 (2001) 2378-2387. 
[51] L. Thrane, R. H. Jacobsen, P. U. Jepsen, S. R. Keiding, THz Reflection Spectroscopy of Liquid Water, Chem. Phys. Lett., 240 (1995) 330-333.

[52] J. T. Kindt, C. A. Schmuttenmaer, Far-Infrared Dielectric Properties of Polar Liquids Probed by Femtosecond Terahertz Pulse Spectroscopy, J. Phys. Chem., 100 (1996) 1037310379 .

[53] D. M. Carey, G. M. Korenowski, Measurement of the Raman Spectrum of Liquid Water, J. Chem. Phys., 108 (1998) 2669-2675.

[54] K. Mizoguchi, Y. Hori, Y. Tominaga, Study on Dynamical Structure in Water and Heavy Water by Low-Frequency Raman Spectroscopy, J. Chem. Phys., 97 (1992) 1961-1968.

[55] G. E. Walrafen, Raman Spectrum of Water: Transverse and Longitudinal Acoustic Modes below $300 \mathrm{~cm}^{-1}$ and Optic Modes above $300 \mathrm{~cm}^{-1}$, J. Phys. Chem., 94 (1990) 2237-2239.

[56] G. M. Hale, M. R. Querry, Optical Constants of Water in the 200-nm to 200- $\mu$ m Wavelength Region, Appl. Opt., 12 (1973) 555-563.

[57] M. N. Afsar, J. B. Hasted, Submillimetre Wave Measurements of Optical Constants of Water at Various Temperatures, Infrared Phys., 18 (1978) 835-841.

[58] J. B. Hasted, S. K. Husain, F. A. M. Frescura, J. R. Birch, Far-Infrared Absorption in Liquid Water, Chem. Phys. Lett., 118 (1985) 622-625.

[59] J. B. Hasted, S. K. Husain, F. A. M. Frescura, J. R. Birch, The Temperature Variation of the Near Millimetre Wavelength Optical Constants of Water, Infrared Phys., 27 (1987) 11-15.

[60] J. E. Bertie, Z. Lan, Infrared Intensities of Liquids XX: The Intensity of the OH Stretching Band of Liquid Water Revisited, and the Best Current Values of the Optical Constants of $\mathrm{H}_{2} \mathrm{O}(\mathrm{l})$ at $25^{\circ} \mathrm{C}$ between 15,000 and $1 \mathrm{~cm}^{-1}$, Appl. Spectrosc., 50 (1996) 1047-1057.

[61] P. A. Madden, R. W. Impey, On the Infrared and Raman Spectra of Water in the Region 5 - $250 \mathrm{~cm}^{-1}$, Chem. Phys. Lett., 123 (1986) 502-506.

[62] V. Mazzacurati, M. A. Ricci, G. Ruocco, M. Sampoli, Low-Frequency Raman Spectra of Liquid Water: A Molecular Dynamics Simulation, Chem. Phys. Lett., 159 (1989) 383.

[63] B. Guillot, A Molecular Dynamics Study of the Far Infrared Spectrum of Liquid Water, J. Chem. Phys., 95 (1991) 1543-1551.

[64] W. B. Bosma, L. E. Fried, S. Mukamel, Simulation of the Intermolecular Vibrational Spectra of Liquid Water and Water Clusters, J. Chem. Phys., 98 (1993) 4413-4421.

[65] I. Ohmine, H. Tanaka, Fluctuation, Relaxations, and Hydration in Liquid Water. Hydrogen-Bond Rearrangement Dynamics, Chem. Rev., 93 (1993) 2545-2566.

[66] P. R. Mason, J. B. Hasted, L. Moore, The Use of Statistical Theory in Fitting Equations to Dielectric Dispersion Data, Adv. Mol. Relax. Proc., 6 (1974) 217-232.

[67] I. Rips, J. Klafter, J. Jortner, Dynamics of Ionic Solvation, J. Chem. Phys., 88 (1988) 3246-3252.

[68] I. Rips, J. Klafter, J. Jortner, Solvation Dynamics in Polar Liquids, J. Chem. Phys., 89 (1988) 4288-4299.

[69] S. Engström, B. Jönsson, R. W. Impey, Molecular Dynamics Simulation of Quadrupole Relaxation of Atomic Ions in Aqueous Solution, J. Chem. Phys., 80 (1984) 5481-5486.

[70] M. Maroncelli, E. W. Castner Jr., B. Bagchi, G. R. Fleming, Dipolar Solvation Dynamics, Faraday Discuss. Chem. Soc., 85 (1988) 199-210. 
[71] M. Maroncelli, G. R. Fleming, Computer Simulation of the Dynamics of Aqueous Solvation, J. Chem. Phys., 89 (1988) 5044-5069.

[72] O. A. Karim, A. D. J. Haymet, M. J. Banet, J. D. Simon, Molecular Aspects of Nonequilibrium Solvation: A Simulation of Dipole Relaxation, J. Phys. Chem., 92 (1988) 3391-3394.

[73] J. S. Bader, D. Chandler, Computer Simulation of Photochemically Induced Electron Transfer, Chem. Phys. Lett., 157 (1989) 501-504.

[74] X. Y. Song, D. Chandler, Dielectric Solvation Dynamics of Molecules of Arbitrary Shape and Charge Distribution, J. Chem. Phys., 108 (1998) 2594-2600.

[75] N. Nandi, S. Roy, B. Bagchi, Ultrafast Solvation Dynamics in Water: Isotope Effects and Comparison with Experimental Results, J. Chem. Phys., 102 (1995) 1390-1397.

[76] M. Cho, G. Fleming, S. Saito, I. Ohmine, R. M. Stratt, Instantaneous Normal Mode Analysis of Liquid Water, J. Chem. Phys., 100 (1994) 6672-6683.

[77] H. Tanaka, I. Ohmine, Potential Energy Surfaces for Water Dynamics: Reaction Coordinates, Transition States, and Normal Mode Analyses, J. Chem. Phys., 91 (1989) 63186327.

[78] R. B. Barnett, U. Landman, A. Nitzan, Relaxation Dynamics Following Transition of Solvated Electrons, J. Chem. Phys., 90 (1989) 4413-4422.

[79] H. Bürsing, S. Kundu, P. Vöhringer, Solvation Dynamics at Aqueous Lipid-Membrane Interfaces Explored by Temperature-Dependent 3-Pulse-Echo Peak Shifts: Influence of the Lipid Polymorphism, J. Phys. Chem. B, 107 (2003) 2404-2414.

[80] S. Vajda, R. Jimenez, S. J. Rosenthal, V. Fidler, G. R. Fleming, E. W. Castner, Femtosecond to Nanosecond Solvation Dynamics in Pure Water and inside the $\gamma$-Cyclodextrin Cavity, J. Chem. Soc., Faraday Trans., 91 (1995) 867-873.

[81] N. Nandi, K. Bhattacharyya, B. Bagchi, Dielectric Relaxation and Solvation Dynamics of Water in Complex Chemical and Biological Systems, Chem. Rev., 100 (2000) 2013-2045.

[82] K. Bhattacharyya, Photophysical Probes for Organized Assemblies, In: V. Ramamurthy, K. S. Schanze (Hrsg.), Organic Molecular Photochemistry, Bd. 3 von Molecular and Supramolecular Photochemistry (Marcel Dekker, New York, 1999), 283-344.

[83] K. Bhattacharyya, B. Bagchi, Slow Dynamics of Constrained Water in Complex Geometries, J. Phys. Chem. A, 104 (2000) 10603-10613.

[84] S. K. Pal, J. Peon, B. Bagchi, A. H. Zewail, Biological Water: Femtosecond Dynamics of Macromolecular Hydration, J. Phys. Chem., 106 (2002) 12376-12395.

[85] C. Tanford, The Hydrophobic Effect: Formation of Micelles and Biological Membranes (John Wiley \& Sons, New York, 1980), 2. Aufl.

[86] Y. Moroi, Micelles: Theoretical and Applied Aspects (Plenum Press, New York, 1992).

[87] H. H. Paradies, Shape and Size of a Nonionic Surfactant Micelle. Triton X-100 in Aqueous Solution, J. Phys. Chem., 84 (1980) 599-607.

[88] G. D. J. Phillies, J. E. Yambert, Solvent and Solute Effects on Hydration and Aggregation Numbers of Triton X-100 Micelles, Langmuir, 12 (1996) 3431-3436.

[89] A. Datta, S. K. Pal, D. Mandal, K. Bhattacharya, Solvation Dynamics of Coumarin 480 in Vesicles, J. Phys. Chem. B, 102 (1998) 6114-6117. 
[90] S. K. Pal, D. Sukul, D. Mandal, K. Bhattacharyya, Solvation Dynamics of DCM in Lipid, J. Phys. Chem. B, 104 (2000) 4529-4531.

[91] S. K. Pal, D. Sukul, D. Mandal, S. Sen, K. Bhattacharyya, Solvation Dynamics of DCM in Dipalmitoyl Phosphatidylcholine Lipid, Tetrahedron, 56 (2000) 6999-7002.

[92] N. Sarkar, K. Das, A. Datta, S. Das, K. Bhatacharyya, Solvation Dynamics of Coumarin 480 in Reverse Micelles. Slow Relaxation of Water Molecules, J. Phys. Chem., 100 (1996) 10523-10527.

[93] S. K. Pal, D. Mandal, D. Sukul, K. Bhattacharyya, Solvation Dynamics of 4(Dicyanomethylene)-2-methyl-6-(p-dimethylaminostyryl)-4H-pyran (DCM) in a Microemulsion, Chem. Phys. Lett., 312 (1999) 178-184.

[94] N. Sarkar, A. Datta, S. Das, K. Bhatacharyya, Solvation Dynamics of Coumarin 480 in Micelles, J. Phys. Chem., 100 (1996) 15483-15486.

[95] A. Datta, D. Mandal, S. K. Pal, S. Das, K. Bhattacharya, Solvation Dynamics in Organized Assemblies. 4-Aminophthalimide in Micelles, J. Mol. Liq., 77 (1998) 121-129.

[96] S. K. Pal, D. Sukul, D. Mandal, S. Sen, K. Bhattacharyya, Solvation Dynamics of DCM in Micelles, Chem. Phys. Lett., 327 (2000) 91-96.

[97] D. Mandal, S. Sen, K. Bhattacharyya, T. Tahara, Femtosecond Study of Solvation Dynamics of DCM in Micelles, Chem. Phys. Lett., 359 (2002) 77-82.

[98] N. Nandi, B. Bagchi, Dielectric Relaxation of Biological Water, J. Phys. Chem. B, 101 (1997) 10954-10961.

[99] R. E. Riter, E. P. Undiks, N. E. Levinger, Impact of the Counterion on Water Motion in Aerosol OT Reverse Micelles, J. Am. Chem. Soc., 120 (1998) 6062-6067.

[100] D. Pant, R. E. Riter, N. E. Levinger, Influence of Restricted Environment and Ionic Interactions on Water Solvation Dynamics, J. Chem. Phys., 109 (1998) 9995-10003.

[101] S. P. Moulik, B. K. Paul, Structure, Dynamics and Transport Properties of Microemulsions, Adv. Colloid Interface Sci., 78 (1998) 99-195.

[102] N. E. Levinger, Ultrafast Dynamics in Reverse Micelles, Microemulsions, and Vesicles, Curr. Opin. Colloid Interface Sci., 5 (2000) 118-124.

[103] P. Hazra, D. Chakrabarty, N. Sarkar, Solvation Dynamics of Coumarin 153 in Aqueous and Non-Aqueous Reverse Micelles, Chem. Phys. Lett., 371 (2003) 553-562.

[104] P. Hazra, D. Chakrabarty, N. Sarkar, Intramolecular Charge Transfer and Solvation Dynamics of Coumarin 152 in Aerosol-OT, Water-Solubilizing Reverse Micelles, and Polar Organic Solvent Solubilizing Reverse Micelles, Langmuir, 18 (2002) 7872-7879.

[105] R. E. Riter, D. M. Willard, N. E. Levinger, Water Immobilization at Surfactant Interfaces in Reverse Micelles, J. Phys. Chem. B, 102 (1998) 2705-2714.

[106] P. Hazra, N. Sarkar, Intramolecular Charge Transfer Processes and Solvation Dynamics of Coumarin 490 in Reverse Micelles, Chem. Phys. Lett., 342 (2001) 303-311.

[107] P. Hazra, D. Chakrabarty, N. Sarkar, Solvation Dynamics of Coumarin 152A in Methanol and Acetonitrile Reverse Micelles, Chem. Phys. Lett., 358 (2002) 523-530.

[108] D. Mandal, A. Datta, S. K. Pal, K. Bhattacharyya, Solvation Dynamics of 4Aminophthalimide in Water-in-Oil Microemulsion of Triton X-100 in Mixed Solvents, J. Phys. Chem., 102 (1998) 9070-9073. 
[109] D. Pant, N. E. Levinger, Polar Solvation Dynamics in Nonionic Reverse Micelles and Model Polymer Solutions, Langmuir, 16 (2000) 10123-10130.

[110] D. M. Willard, N. E. Levinger, Influence of Morphology on Polar Solvation Dynamics in Lecithin Reverse Micelles, J. Phys. Chem. B, 104 (2000) 11075-11080.

[111] D. M. Willard, R. E. Riter, N. E. Levinger, Dynamics of Polar Solvation in Lecithin/Water/Cyclohexane Reverse Micelles, J. Am. Chem. Soc., 120 (1998) 4151-4160.

[112] P. Hazra, N. Sarkar, Solvation Dynamics of Coumarin 490 in Methanol and Acetonitrile Reverse Micelles, Phys. Chem. Chem. Phys., 4 (2000) 1040-1045.

[113] H. Shirota, K. Horie, Solvation Dynamics in Nonaqueous Reverse Micelles, J. Phys. Chem. B, 103 (1999) 1437-1443.

[114] R. E. Riter, E. P. Undiks, J. R. Kimmel, N. E. Levinger, Formamide in Reverse Micelles: Restricted Environment Effects on Molecular Motion, J. Phys. Chem. B, 102 (1998) 7931-7938.

[115] A. Maitra, Determination of Size Parameters of Water-Aerosol OT-Oil Reverse Micelles from Their Nuclear Magnetic Resonance Data, J. Phys. Chem., 88 (1984) 5122-5125.

[116] D. M. Mittelman, M. C. Nuss, V. L. Colvin, Terahertz Spectroscopy of Water in Inverse Micelles, Chem. Phys. Lett., 275 (1997) 332-338.

[117] G.-W. Zhou, G.-Z. Li, W.-J. Chen, Fourier Transform Infrared Investigation on Water States and the Conformations of Aerosol-OT in Reverse Microemulsions, Langmuir, 18 (2002) 4566-4571.

[118] C. Boissière, J. B. Brubach, A. Mermet, G. de Marzi, C. Bourgaux, E. Prouzet, P. Roy, Water Confined in Lamellar Structures of AOT Surfactants: An Infrared Investigation, J. Phys. Chem. B, 106 (2002) 1032-1035.

[119] P. D. Moran, G. A. Bowmaker, R. P. Cooney, J. R. Bertlett, J. L. Woolfrey, Vibrational Spectra of Metal Salts of Bis(2-ethylhexyl)sulfosuccinate (AOT), J. Mater. Chem., 5 (1995) 295-302.

[120] H. Hauser, G. Haering, A. Pande, P. Luisi, Interaction of Water with Sodium Bis(2-ethyl1-hexyl) Sulfosuccinate in Reversed Micelles, J. Phys. Chem., 93 (1989) 7869-7876.

[121] C. A. Martin, L. J. Magid, Carbon-13 NMR Investigations of Aerosol OT Water-in-Oil Microemulsions, J. Phys. Chem., 85 (1981) 3938-3944.

[122] M. Zulauf, H.-F. Eicke, Inverted Micelles and Microemulsions in the Ternary System $\mathrm{H}_{2} \mathrm{O} /$ Aerosol-OT/Isooctane as Studied by Photon Correlation Spectroscopy, J. Phys. Chem., 83 (1979) 480-486.

[123] K. K. Karukstis, A. A. Frazier, C. T. Loftus, A. S. Tuan, Fluorescence Investigation of Multiple Partitioning Sites in Aqueous and Reverse Micelles, J. Phys. Chem. B, 102 (1998) 8163-8169.

[124] M. D'Angelo, D. Fioretto, G. Onori, A. Santucci, Micellar Interactions in Water-in-Oil Microemulsions, J. Mol. Struct., 383 (1996) 157-163.

[125] J. Faeder, B. Ladanyi, Molecular Dynamics Simulations of the Interior of Aqueous Reverse Micelles, J. Phys. Chem. B, 104 (2000) 1033-1046.

[126] N. E. Levinger, Water in Confinement, Science, 298 (2002) 1722-1723.

[127] B. B. Raju, S. M. B. Costa, Nanosecond Time Resolved Emission Spectroscopy of Aminocoumarins in AOT Reversed Micelles, Phys. Chem. Chem. Phys., 1 (1999) 5029-5034. 
[128] D. Brown, J. H. R. Clarke, Molecular Dynamics Simulation of a Model Reverse Micelle, J. Phys. Chem., 92 (1988) 2881-2888.

[129] P. Linse, Molecular Dynamics Study of the Aqueous Core of a Reversed Ionic Micelle, J. Chem. Phys., 90 (1989) 4992-5004.

[130] D. J. Tobias, M. L. Klein, Molecular Dynamics Simulations of a Calcium Carbonate / Calcium Sulfonate Reversed Micelle, J. Phys. Chem., 100 (1996) 6637-6648.

[131] J. Faeder, B. M. Ladanyi, Solvation Dynamics in Aqueous Reverse Micelles: A Computer Simulation Study, J. Phys. Chem. B, 105 (2001) 11148-11158.

[132] R. Hutterer, F. W. Schneider, N. Pérez, H. Ruf, M. Hof, Influence of Lipid Composition and Membrane Curvature on Fluorescence and Solvent Relaxation Kinetics in Unilamellar Vesicles, J. Fluoresc., 3 (1993) 257-259.

[133] M. Hof, R. Hutterer, N. Perez, H. Ruf, F. W. Schneider, Influence of Vesicle Curvature on Fluorescence Relaxation Kinetics of Fluorophores, Biophys. Chem., 52 (1994) 165-172.

[134] R. Hutterer, F. W. Schneider, H. Sprinz, M. Hof, Binding and Relaxation Behaviour of Prodan and Patman in Phospholipid Vesicles: A Fluorescence and ${ }^{1} H$ NMR Study, Biophys. Chem., 61 (1996) 151-160.

[135] R. Hutterer, F. W. Schneider, M. Hof, Time Resolved Emission Spectra and Anisotropy Profiles for Symmetric Diacyl- and Dietherphosphatidylcholines, J. Fluoresc., 7 (1997) 27-33.

[136] R. Hutterer, F. W. Schneider, H. Lanig, M. Hof, Solvent Relaxation Behaviour of $n$ Anthroyloxy Fatty Acids in PC-Vesicles and Paraffin Oil: A Time-Resolved Emission Spectra Study, Biochim. Biophys. Acta, 1323 (1997) 195-207.

[137] R. Hutterer, F. W. Schneider, V. Fidler, E. Grell, M. Hof, Time-Evolved Emission Spectra of Prodan and Patman in Large Unilamellar Vesicles: A Comparison between Dietherand Diacyllipids, J. Fluoresc., 7 (1997 (Supplement)) 161S-163S.

[138] R. Hutterer, A. B. J. Parusel, M. Hof, Solvent Relaxation of Prodan and Patman: A Useful Tool for the Determination of Polarity and Rigidity Changes in Membranes, J. Fluoresc., 8 (1998) 389-393.

[139] J. Sýkora, P. Kapusta, V. Fidler, M. Hof, On What Time Scale Does Solvent Relaxation in Phospholipid Bilayers Happen?, Langmuir, 18 (2002) 571-574.

[140] T. Parasassi, G. D. Stasio, A. d'Ubaldo, E. Gratton, Phase Fluctuation in Phospholipid Membranes Revealed by Laurdan Fluorescence, Biophys. J., 57 (1990) 1179-1186.

[141] T. Parasassi, G. Ravagnan, R. M. Rusch, E. Gratton, Modulation and Dynamics of Phase Properties in Phospholipid Mixtures Detected by Laurdan Fluorescence, Photochem. Photobiol., 57 (1993) 403-410.

[142] T. Parasassi, E. K. Krasnowska, L. Bagatolli, E. Gratton, Laurdan and Prodan as Polarity-Sensitive Fluorescent Membrane Probes, J. Fluoresc., 8 (1998) 365-373.

[143] H. Bürsing, S. Kundu, P. Vöhringer, Probing Solvation Dynamics at Aqueous LipidMembrane Interfaces with 3-Pulse Photon-Echo Peak-Shifts, In: A. Douhal, J. Santamaria (Hrsg.), Femtochemistry and Femtobiology: Ultrafast Dynamics in Molecular Science (World Scientific, Singapore, 2002), 674-680.

[144] R. Koynova, M. Caffrey, Phases and Phase Transitions of the Phosphatidylcholines, Biochim. Biophys. Acta, 1376 (1998) 91-145. 
[145] Y. Inoko, T. Mitsui, Structural Parameters of Dipalmtoyl Phosphatidylcholine Lamellar Phases and Bilayer Phase Transitions, J. Phys. Soc. Jpn., 44 (1978) 1918-1924.

[146] G. Cevc, Polymorphism of the Bilayer Membranes in the Ordered Phase and the Molecular Origin of the Lipid Pretransition and Rippled Lamellae, Biochim. Biophys. Acta, 1062 (1991) 59-69.

[147] T. L. Bihan, M. Pezolet, Study of the Structure and Phase Behavior of Dipalmitoylphosphatidylcholine by Infrared Spectroscopy - Characterization of the Pretransition and Subtransition, Chem. Phys. Lipids, 94 (1998) 13-33.

[148] H. Heller, M. Schaefer, K. Schulten, Molecular Dynamics Simulation of a Bilayer of 200 Lipids in the Gel and in the Liquid-Crystal Phases, J. Phys. Chem., 97 (1993) 8343-8360.

[149] F. Zhou, K. Schulten, Molecular Dynamics Study of a Membrane-Water Interface, J. Phys. Chem., 99 (1995) 2194-2207.

[150] H. E. Alper, D. Bassolino-Klimas, T. R. Stouch, The Limiting Behavior of Water Hydrating a Phospholipid Monolayer: A Computer Simulation Study, J. Chem. Phys., 99 (1993) 5547-5559.

[151] H. E. Alper, D. Bassolino, T. R. Stouch, Computer Simulation of a Phospholipid Monolayer-Water System: The Influence of Long Range Forces on Water Structure and Dynamics, J. Chem. Phys., 98 (1993) 9798-9807.

[152] W. Shinoda, M. Shimizu, S. Okazaki, Molecular Dynamics Study on Electrostatic Properties of a Lipid Bilayer: Polarization, Electrostatic Potential, and the Effects on Structure and Dynamics of Water near the Interface, J. Phys. Chem. B, 102 (1998) 6647-6654.

[153] D. P. Tieleman, S. J. Marrink, H. J. C. Berendsen, A Computer Perspective of Membranes: Molecular Dynamics Studies of Lipid Bilayer Systems, Biochim. Biophys. Acta, 1331 (1997) 235-270.

[154] K. Tu, D. J. Tobias, M. L. Klein, Constant Pressure and Temperature Molecular Dynamics Simulation of a Fully Hydrated Liquid Crystal Phase Dipalmitoylphosphatidylcholine Bilayer, Biophys. J., 69 (1995) 2558-2562.

[155] K. Tu, D. J. Tobias, M. L. Klein, Constant Pressure and Temperature Molecular Dynamics Simulations of Crystals of the Lecithin Fragments: Glycerylphosphorylcholine and Dilauroylglycerol, J. Phys. Chem., 99 (1995) 10035-10042.

[156] G. Weber, F. J. Farris, Synthesis and Spectral Properties of a Hydrophobic Fluorescent Probe: 6-Propionyl-2-(dimethylamino)naphthalene, Biochemistry, 18 (1979) 3075-3078.

[157] J. Catalan, P. Perez, J. Laynez, F. G. Blanco, Analysis of the Solvent Effect on the Photophysics Properties of 6-Propionyl-2-(dimethylamino)naphthalene (Prodan), J. Fluoresc., 1 (1991) 215-223.

[158] B. Valeur, Molecular Fluorescence - Principles and Applications (Wiley-VCH, Weinheim, 2002).

[159] A. Balter, W. Nowak, W. Pawelkiewicz, A. Kowalczyk, Some Remarks on the Interpretation of the Spectral Properties of Prodan, Chem. Phys. Lett., 143 (1988) 565-570.

[160] C. E. Bunker, T. L. Bowen, Y.-P. Sun, A Photophysical Study of Solvatochromic Probe 6-Propionyl-2-(N,N-dimethylamino)naphthalene (Prodan) in Solution, Photochem. Photobiol., 58 (1993) 499-505. 
[161] W. Baumann, Z. Nagy, A. K. Maiti, H. Reis, S. V. Rodrigues, N. Detzer, Intramolecular Charge Transfer as Revealed by Results from the Measurement of Ground and Excited State Dipole Moments, In: N. Mataga, T. Okada, H. Masuhara (Hrsg.), Dynamics and Mechanisms of Photoinduced Electron Transfer and Related Phenomena, Bd. 29 (Elsevier Science Publishers B. V., Amsterdam, 1992), 211-229.

[162] A. Kawski, B. Kuklinski, P. Bojarski, H. Diehl, Ground and Excited State Dipole Moments of Laurdan determined from Solvatochromic and Thermochromic Shifts of Absorption and Fluorescence Spectra, Z. Naturforsch., 55a (2000) 817-822.

[163] P. L. G. Chong, Effects of Hydrostatic-Pressure on the Location of Prodan in Lipid Bilayers and Cellular Membranes, Biochemistry, 27 (1988) 399-404.

[164] R. B. Macgregor Jr., G. Weber, Fluorophores in Polar Media: Spectral Effects of the Langevin Distribution of Electrostatic Interactions, In: H. Morawetz, I. Steinberg (Hrsg.), Ann. N.Y. Acad. Sci., Bd. 366 (New York Academy of Sciences, New York, 1981), 140154.

[165] L. A. Bagatolli, T. Parasassi, G. D. Fidelio, E. Gratton, A Model for the Interaction of 6-Lauroyl-2-(N,N-dimethylamino)naphthalene with Lipid Environments: Implications for Spectral Properties, Photochem. Photobiol., 70 (1999) 557-564.

[166] T. Parasassi, G. D. Stasio, G. Ravagnan, R. M. Rusch, E. Gratton, Quantitation of Lipid Phases in Phospholipid-Vesicles by the Generalized Polarization of Laurdan Fluorescence, Biophys. J., 60 (1991) 179-189.

[167] W. Nowak, P. Adamczak, A. Balter, A. Sygula, On the Possibility of Fluorescence from Twisted Intramolecular Charge Transfer States of 2-Dimethylamino-6-acylnaphthalenes. A Quantum-Chemical Study, J. Mol. Struct. (Theochem), 139 (1986) 13-23.

[168] K. Bhattacharyya, M. Chowdhury, Environmental and Magnetic Field Effects on Exciplex and Twisted Charge Transfer Emission, Chem. Rev., 93 (1993) 507-535.

[169] A. B. J. Parusel, W. Nowak, S. Grimme, G. Köhler, Comparative Theoretical Study on Charge-Transfer Fluorescence Probes: 6-Propanoyl-2-(N,N-dimethylamino)naphthalene and Derivates, J. Phys. Chem. A, 102 (1998) 7149-7156.

[170] A. Parusel, Semiempirical Studies of Solvent Effects on the Intramolecular Charge Transfer of the Fluorescence Probe Prodan, J. Chem. Soc., Faraday Trans., 94 (1998) 29232927.

[171] M. Viard, J. Gallay, M. Vincent, O. Meyer, B. Robert, M. Paternostre, Laurdan Solvatochromism: Solvent Dielectric Relaxation and Intramolecular Excited-State Reaction, Biophys. J., 73 (1997) 2221-2234.

[172] U. Brackmann, Lambdachrome ${ }^{\circledR}$ Laser Dyes (Lambda Physik Lasertechnik, Göttingen, 1985), 2. Aufl.

[173] I. Martini, G. V. Hartland, Relaxation Dynamics in the First Excited Singlet State of Cyanine Dye: HITC, Chem. Phys. Lett., 258 (1996) 180-186.

[174] I. Martini, G. V. Hartland, Ultrafast Investigation of Vibrational Relaxation in the $S_{1}$ Electronic State of HITC, J. Phys. Chem., 100 (1996) 19764-19770.

[175] Shimadzu Corporation Kyoto Japan, UV-VIS Recording Spectrophotometer UV-160 Instruction Manual.

[176] Jobin Yvon - Spex Instruments S. A. Inc., Fluorolog-3 with DataMax for Windows ${ }^{T M}$ Operation and Maintenance Manual (1996). 
[177] G. Schwedt, Analytische Chemie - Grundlagen, Methoden, Praxis (Thieme, Stuttgart, 1995).

[178] D. V. O'Conner, D. Phillips, Time-Correlated Single Photon Counting (Acedemic Press, London, 1984).

[179] J. Jethwa, P. Vöhringer, Unpublizierter Aufbau.

[180] A. Hessel, A. A. Oliner, A New Theory of Wood's Anomalies on Optical Gratings, Appl. Opt., 4 (1965) 1275-1297.

[181] P. Murdin, Wood's Anomalies in the INT Spectrograph, ING La Palma Technical Note No. 76, (1990).

[182] C. Palmer, Diffraction Grating Handbook (Richardson Grating Laboratory, New York, 2000), 4. Aufl.

[183] Edinburgh Instruments Ltd., TCC 900 Computer Module for Time Correlated Single Photon Counting (2001), 1. Aufl.

[184] M. Wahl, R. Erdmann, Time Correlated Single Photon Counting in Fluorescence Lifetime Analysis - Part 1. Basics, Hardware and Experimental Setup, Photonik, 1 (2000) 14-19.

[185] M. Wahl, R. Erdmann, Time Correlated Single Photon Counting in Fluorescence Lifetime Analysis - Part 2. Applications, Data Analysis and Interpretation, Photonik, 2 (2000) 1619.

[186] K. Winkler, Ultraschnelle, lichtinduzierte Primärprozesse im elektronisch angeregten Zustand des Grün Fluoreszierenden Proteins (GFP), Dissertation, Universität Göttingen (2002).

[187] D. B. Siano, D. E. Metzler, Band Shapes of Electronic Spectra of Complex Molecules, J. Chem. Phys., 51 (1969) 1856-1861.

[188] B. Röder, Einführung in die molekulare Photobiophysik (Teubner, Stuttgart, 1999).

[189] Clark-MXR Inc., CPA-2001 User's Manual Version 1.0 (1998).

[190] M. Kling, Experimentelle und Theoretische Untersuchungen der Femtosekunden-Kinetik des Photoinduzierten Zerfalls Aromatischer Peroxyverbindungen, Dissertation, Universität Göttingen (2002).

[191] K. Tamura, E. P. Ippen, H. A. Haus, Pulse Dynamics in Stretched-Pulse Fiber Lasers, Appl. Phys. Lett., 67 (1995) 158-160.

[192] K. Tamura, M. Nakazawa, Optimizing Power Extraction in Stretched-Pulse Fiber Ring Lasers, Appl. Phys. Lett., 67 (1995) 3691-3693.

[193] P. Maine, D. Strickland, P. Bado, M. Pesset, G. Mourou, Generation of Ultrahigh Peak Power Pulses by Chirped Pulse Amplification, IEEE J. Quantum Electron., 24 (1988) 398-403.

[194] P. Bado, M. Bouvier, J. S. Coe, Nd:YLF Mode-Locked Oscillator and Regenerative Amplifier, Opt. Lett., 12 (1987) 319-321.

[195] D. Du, J. Squier, S. Kane, G. Korn, G. Mourou, C. Bogusch, C. T. Cotton, Terawatt Ti:Sapphire Laser with a Spherical Reflective-Optic Pulse Expander, Opt. Lett., 20 (1995) $2114-2116$.

[196] D. Strickland, G. Mourou, Compression of Amplified Chirped Optical Pulses, Opt. Commun., 56 (1985) 219-221. 
[197] Stanford Research Systems, 1998 - 1999 Scientific and Engineering Instruments (1998).

[198] W. Demtröder, Laserspektroskopie - Grundlagen und Techniken (Springer, Berlin, 2000), 4. Aufl.

[199] P. Horowitz, W. Hill, The Art of Electronics (Cambridge University Press, Cambridge, 1981).

[200] M. A. Duguay, J. W. Hansen, An Ultrafast Gate, Appl. Phys. Lett., 15 (1969) 192-194.

[201] J. Takeda, K. Nakajima, S. Kurita, S. Tomimoto, S. Saito, T. Suemoto, Femtosecond Optical Kerr Gate Fluorescence Spectroscopy for Ultrafast Relaxation Processes, J. Lumin., 87-89 (2000) 927-929.

[202] Y. Kanematsu, H. Ozawa, I. Tanaka, S. Kinoshita, Femtosecond Optical Kerr-Gate Measurement of Fluorescence Spectra of Dye Solutions, J. Lumin., 87-89 (2000) 917-919.

[203] H. Mahr, M. D. Hirsch, Optical Up-Conversion Light Gate With Picosecond Resolution, Opt. Commun., 13 (1975) 96-99.

[204] M. A. Kahlow, W. Jarzȩba, T. P. DuBruil, P. F. Barbara, Ultrafast Emission-Spectroscopy in the Ultraviolet by Time-Gated Upconversion, Rev. Sci. Instrum., 59 (1988) 1098-1109.

[205] J. Shah, Ultrafast Luminescence Spectroscopy using Sum Frequency Generation, IEEE J. Quantum Electron., 24 (1988) 276-288.

[206] J.-C. Mialocq, T. Gustavsson, Investigations of Femtosecond Chemical Reactivity by Means of Fluorescence Up-Conversion, In: B. Valeur, J.-C. Brochon (Hrsg.), New Trends in Fluorescence Spectroscopy - Applications to Chemical and Life sciences, Bd. 1 von Springer Series on Fluorescence, Kap. 4 (Springer, Berlin, 2001), 61-80.

[207] F. Zernike, J. E. Midwinter, Applied Nonlinear Optics (John Wiley \& Sons, New York, 1973).

[208] C. Rullière (Hrsg.), Femtosecond Laser Pulses - Principles and Experiments (Springer, Berlin, 1998).

[209] T. Steinel, Untersuchungen zur Reaktionsdynamik von Diphenylpolyenen in Lösung mittels Femtosekunden Fluoreszenz-Konversions-Spektroskopie, Dissertation, Universität Göttingen (2000).

[210] N. Breidenassel, Femtosekunden-Spektroskopie zur Protonentransfer-Dynamik im elektronisch angeregten Zustand von 1,8-Dihydroxy-9,10-Anthrachinon, Dissertation, Universität Göttingen (2003).

[211] C. V. Shank, A. Dienes, A. M. Trozzolo, J. A. Myer, Near UV to Yellow Tunable Laser Emission from an Organic Dye, Appl. Phys. Lett., 16 (1970) 405-407.

[212] A. Dienes, C. V. Shank, R. L. Kohn, Characteristics of 4-Methylumbelliferone Laser Dye, IEEE J. Quantum Electron., QE-9 (1973) 833-843.

[213] T. Wilhelm, J. Piel, E. Riedle, Sub-20fs Pulses Tunable across the Visible from a BluePumped Single-Pass Noncollinear Parametric Converter, Opt. Lett., 22 (1997) 1494-1496.

[214] E. Riedle, M. Beutter, S. Lochbrunner, J. Piel, S. Schenkl, S. Spörlein, W. Zinth, Generation of 10 to $50 \mathrm{fs}$ Pulses Tunable through all of the Visible and the NIR, Appl. Phys. $B, 71$ (2000) 457-465.

[215] J. Piel, M. Beutter, E. Riedle, 20-50-fs Pulses Tunable across the Near Infrared from a Blue-Pumped Noncollinear Parametric Amplifier, Opt. Lett., 25 (2000) 180-182. 
[216] F. Salin, J. Watson, J.-F. Cormier, P. Georges, A. Brun, Physical Origins of the Spectral Continuum: Self-Focusing, Self-Trapping and Cerenkov Radiation, In: J.-L. Martin, A. Migus, G. A. Mourou, A. H. Zewail (Hrsg.), Ultrafast Phenomena VIII, Bd. 55 von Springer Series in Chemical Physics (Springer, Berlin, 1993), 306-308.

[217] M. K. Reed, M. K. Steiner-Shepard, D. K. Negus, Widely Tunable Femtosecond Optical Parametric Amplifier at $250 \mathrm{kHz}$ with a Ti:Sapphire Regenerative Amplifier, Opt. Lett., 19 (1994) 1855-1857.

[218] M. K. Reed, M. K. Steiner-Shepard, M. S. Armas, D. K. Negus, Microjoule-Energy Ultrafast Optical Parametric Amplifiers, J. Opt. Soc. Am. B, 12 (1995) 2229-2236.

[219] Z. Bor, B. Rácz, Group Velocity Dispersion in Prisms and its Application to Pulse Compression and Travelling-Wave Excitation, Opt. Commun., 54 (1985) 165-170.

[220] W. Rudolph, B. Wilhelmi, Light Pulse Compression, Bd. 3 von Laser Science and Technology - An Interntional Handbook (Harwood Academic Publishers, Chur, 1989).

[221] R. C. MacDonald, R. I. MacDonald, B. P. Menco, K. Takeshita, N. K. Subbarao, L. Hu, Small-Volume Extrusion Apparatus for Preparation of Large, Unilamellar Vesicles, Biochim. Biophys. Acta, 1061 (1991) 297-203.

[222] P. Ilich, F. G. Prendergast, Singlet Adiabatic States of Solvated Prodan - A Semiempirical Molecular-Orbital Study, J. Phys. Chem., 93 (1989) 4441-4447.

[223] R. C. Weast (Hrsg.), CRC Handbook of Chemistry and Physics - A Ready-Reference Book of Chemical and Physical Data (CRC Press, Boca Raton, 1983), 64. Aufl.

[224] R. M. Epand, R. Kraayenhof, Fluorescent Probes used to Monitor Membrane Interfacial Polarity, Chem. Phys. Lipids, 101 (1999) 57-64.

[225] T. Heimburg, Mechanical Aspects of Membrane Thermodynamics. Estimation of the Mechanical Properties of Lipid Membranes close to the Chain Melting Transition from Calorimetry, Biochim. Biophys. Acta, 1415 (1998) 147-162.

[226] G. Mie, Beiträge zur Optik trüber Medien, speziell kolloidaler Metallösungen, Ann. d. Phys., 25 (1908) 377-445.

[227] Ph. Wahl, Fluorescence Anisotropy of Chromophores Rotating between Two Reflecting Barriers, Chem. Phys., 7 (1975) 210-219.

[228] Ph. Wahl, Theoretical Determination of Decay, Quantum Yield and Anisotropy of Chromophores Attached to Macromolecules and Performing a Local Brownian Motion, Chem. Phys., 7 (1975) 220-228.

[229] K. Kinosita Jr., S. Kawato, A. Ikegami, A Theory of Fluorescence Polarization Decay in Membranes, Biophys. J., 20 (1977) 289-305.

[230] G. Lipari, A. Szabo, Effect of Librational Motion on Fluorescence Depolarization and Nuclear Magnetic Resonance Relaxation in Macromolecules and Membranes, Biophys. J., 30 (1980) 489-506.

[231] S. Kawato, K. Kinosita Jr., A. Ikegami, Dynamic Structure of Lipid Bilayers Studied by Nanosecond Fluorescence Techniques, Biochemistry, 16 (1977) 2319-2324.

[232] K. Kinosita Jr., S. Kawato, A. Ikegami, S. Yoshida, Y. Orii, The Effect of Cytochrome Oxidase on Lipid Chain Dynamics: A Nanosecond Fluorescence Depolarization Study, Biochim. Biophys. Acta, 647 (1981) 7-17. 
[233] P. A. van Paridon, J. K. Shute, K. W. A. Wirtz, A. J. W. G. Visser, A Fluorescence Decay Study of Parinaroyl-Phosphatidylinositol Incorporated into Artificial and Natural Membranes, Eur. Biophys. J., 16 (1988) 53-63.

[234] G. Schröder, Molekulardynamiksimulationen der Flexibilität und Fluoreszenzanisotropie eines an ein Protein gebundenen Farbstoffs, Diplomarbeit, Universität Göttingen (2000).

[235] C. Zannoni, A. Arcioni, P. Cavatorta, Fluorescence Depolarization in Liquid Crystals and Membrane Bilayers, Chem. Phys. Lipids, 32 (1983) 179-250.

[236] W. V. der Meer, H. Pottel, W. Herremann, M. Ameloot, H. Hendrickx, H. Schröder, Effect of Orientational Order on the Decay of the Fluorescence Anisotropy in Membrane Suspensions. A new Approximate Solution of the Rotational Diffusion Equation, Biophys. J., 46 (1984) 515-523.

[237] A. Szabo, Theory of Fluorescence Depolarization in Macromolecules and Membranes, J. Chem. Phys., 81 (1984) 150-167.

[238] M. Maroncelli, G. R. Fleming, Picosecond Solvation Dynamics of Coumarin-153 - The Importance of Molecular Aspects of Solvation, J. Chem. Phys., 86 (1987) 6221-6239.

[239] I. N. Bronstein, K. A. Semendjajew, Taschenbuch der Mathematik (Verlag Harri Deutsch, Thun, 1981), 19. Aufl.

[240] D. Bingemann, Untersuchung der molekularen Solvatationsdynamik in dielektrischen Flüssigkeiten mittels Femtosekunden-Absorptions- und Verstärkungsspektroskopie, Dissertation, Universität Göttingen (1994).

[241] D. Bingemann, N. P. Ernsting, Femtosecond Solvation Dynamics determining the Band Shape of Stimulated Emission from a Polar Styryl Dye, J. Chem. Phys., 102 (1995) 2691-2700.

[242] M. L. Horng, J. A. Gardecki, A. Papazyan, M. Maroncelli, Subpicosecond Measurements of Polar Solvation Dynamics - Coumarin 153 Revisited, J. Phys. Chem., 99 (1995) 1731117337.

[243] T. Kühne, Untersuchung der Solvatationsdynamik von elektronisch angeregtem 4-(N,NDimethylamino)-4'-cyanostilben in polaren Lösungsmitteln durch nichtlineares optisches Frequenzmischen der spontanen Fluoreszenz, Diplomarbeit, Universität Göttingen (1995).

[244] R. M. Lynden-Bell, I. R. McDonald, Reorientational Correlation Functions for ComputerSimulated Liquids of Tetrahedral Molecules, Mol. Phys., 43 (1981) 1429-1440.

[245] R. M. Lynden-Bell, I. R. McDonald, Tests of Models of Molecular Reorientation in Liquids, Chem. Phys. Lett., 89 (1982) 105-109.

[246] R. M. Lynden-Bell, W. A. Steele, A Model for Strongly Hindered Molecular Reorientation in Liquids, J. Phys. Chem., 88 (1984) 6514-6518.

[247] H. Kunieda, K. Shinoda, Solution Behavior of Aerosol OT/Water/Oil System, J. Colloid Interface Sci., $\mathbf{7 0}$ (1979) 577.

[248] R. Kubo, M. Toda, N. Hashitsume, Statistical Physics II - Nonequilibrium Statistical Mechanics, Bd. 31 von Springer Series in Solid State Sciences (Springer, Berlin, 1991), 2. Aufl.

[249] M. Maroncelli, G. R. Fleming, Comparison of Time-Resolved Fluorescence Stokes Shift Measurements to a Molecular Theory of Solvation Dynamics, J. Chem. Phys., 89 (1988) $875-881$. 
[250] V. Mazzacurati, A. Nucara, M. A. Ricci, G. Ruocco, G. Signorelli, High-Resolution LowFrequency Raman Spectra of Liquid $\mathrm{H}_{2} \mathrm{O}$ and $D_{2} \mathrm{O}$, J. Chem. Phys., 93 (1990) 7767-7773.

[251] X. Y. Song, D. Chandler, R. A. Marcus, Gaussian Field Model of Dielectric Solvation Dynamics, J. Phys. Chem., 100 (1996) 11954-11959.

[252] C. P. Hsu, X. Y. Song, R. A. Marcus, Time-Dependent Stokes Shift and its Calculation from Solvent Dielectric Dispersion Data, J. Phys. Chem. B, 101 (1997) 2546-2551.

[253] C. J. Montrose, J. A. Bucaro, J. Mashall-Coakley, T. A. Litovitz, Depolarized Rayleigh Scattering and Hydrogen Bonding in Liquid Water, J. Chem. Phys., 60 (1974) 5025-5029.

[254] K. Winkler, J. Lindner, V. Subramaniam, T. M. Jovin, P. Vöhringer, Ultrafast Dynamics in the Excited State of Green Fluorescent Protein (wt) studied by Frequency-Resolved Femtosecond Pump-Probe Spectroscopy, Phys. Chem. Chem. Phys., 4 (2002) 1072-1081.

[255] C. Rønne, P. O. Astrand, S. R. Keiding, THz Spectroscopy of Liquid $\mathrm{H}_{2} \mathrm{O}$ and $\mathrm{D}_{2} \mathrm{O}$, Phys. Rev. Lett., 82 (1999) 2888-2891.

[256] C. Rønne, L. Thrane, P. O. Åstrand, A. Wallqvist, K. Mikkelsen, S. R. Keiding, Investigation of the Temperature Dependence of Dielectric Relaxation in Liquid Water by THz Reflection Spectroscopy and Molecular Dynamics Simulation, J. Chem. Phys., 107 (1997) 5319-5331.

[257] S. Woutersen, H. J. Bakker, Resonant Intermolecular Transfer of Vibrational Energy in Liquid Water, Nature, 402 (1999) 507-509.

[258] V. Nagarajan, A. M. Brearley, T.-J. Kang, P. F. Barbara, Time-Resolved Spectroscopic Measurements on Microscopic Solvation Dynamics, J. Chem. Phys., 86 (1987) 3183-3196.

[259] W. P. de Boeij, M. S. Pshenichnikov, D. A. Wiersma, Mode Suppression in the NonMarkovian Limit by Time-Gated Stimulated Photon Echo, J. Chem. Phys., 105 (1996) 2953-2960.

[260] E. Lang, H.-D. Lüdemann, Pressure and Temperature Dependence of the Longitudinal Proton Relaxation Times in Supercooled Water to $-87^{\circ} \mathrm{C}$ and 2500 bar, J. Chem. Phys., 67 (1977) 718-720.

[261] E. Lang, H.-D. Lüdemann, Pressure and Temperature Dependence of the Longitudinal Deuterium Relaxation Times in Supercooled Heavy Water to $300 \mathrm{MPa}$ and $188 \mathrm{~K}, \mathrm{Ber}$. Bunsen-Ges. Phys. Chem., 84 (1980) 462-470.

[262] E. W. Lang, H.-D. Lüdemann, Anomalies of Liquid Water, Angew. Chem. Int. Ed. Engl., 21 (1982) 315-329.

[263] D. Pant, N. E. Levinger, Polar Solvation Dynamics of $\mathrm{H}_{2} \mathrm{O}$ and $\mathrm{D}_{2} \mathrm{O}$ at the Surface of Zirconia Nanoparticles, J. Phys. Chem. B, 103 (1999) 7846-7852.

[264] J. Chesnoy, A. Mokhtari, Resonant Impulsive-Stimulated Raman Scattering on Malachite Green, Phys. Rev. A, 38 (1988) 3566-3576.

[265] H. Barkhuijsen, R. de Beer, W. M. M. J. Bovée, D. V. Ormondt, Retrieval of Frequencies, Amplitudes, Damping Factors, and Phases from Time-Domain Signals Using a Linear Least-Squares Procedure, J. Magn. Reson., 61 (1985) 465-481.

[266] H. Sato, M. Kawasaki, K. Kasatani, M. Katsumata, Raman Spectra of Some Indo-, Thiaand Selena-Carbocyanine Dyes, J. Raman Spectrosc., 19 (1988) 129-132.

[267] K. Iwata, W. L. Weaver, T. L. Gustafson, Spontaneous Raman Spectra of the Cyanine Dye DODCI and Its Six Analogues Using Titanium:Sapphire Laser Excitation, J. Phys. Chem., 96 (1992) 10219-10224. 
[268] J.-P. Yang, R. H. Callender, The Resonance Raman Spectra of Some Cyanine Dyes, J. Raman Spectrosc., 16 (1985) 319-321. 


\section{Abbildungsverzeichnis}

2.1 Dipolare Solvatation . . . . . . . . . . . . . . . . . . 9 9

2.2 Zeitabhängiger Fluoreszenz Stokes-Shift . . . . . . . . . . . . . . . 10

2.3 Pulssequenz für das 3-Puls Photon-Echo Peakshift Experiment . . 13

2.4 Feynman-Diagramme . . . . . . . . . . . . . . . . . . . 14

3.1 Aggregate amphiphiler Moleküle in Wasser . . . . . . . . . . . . 22

3.2 Chemische Strukturen typischer Detergentien . . . . . . . . . 29

3.3 Schematischer Aufbau einer inversen Mizelle . . . . . . . . . . . . 31

3.4 Phasenzustände in Lipidmembranen . . . . . . . . . . . . . . . . 37

3.5 Chemische Struktur der Farbstoffe Prodan und Laurdan . . . . . 43

3.6 Schema einer mit Laurdan markierten DMPC-Membran . . . . . 45

3.7 Chemische Struktur des Farbstoffes HIDCI . . . . . . . . . . . . . 46

4.1 Schematischer Aufbau des TCSPC-Experimentes . . . . . . . . 50

4.2 Gerätefunktion der TCSPC-Apparatur . . . . . . . . . . . . 52

4.3 Verstärkung der Laserpulse im RGA . . . . . . . . . . . . 55

4.4 Autokorrelation der Laserpulse aus dem CPA-2001 . . . . . . . 55

4.5 Prinzip eines Fluoreszenzkonversionsexperimentes . . . . . . . . 57

4.6 Schematischer Aufbau des Fluoreszenzkonversionsexperimentes . . 62

4.7 Kreuzkorrelation der Anregungspulse und der Gatepulse . . . . . 64

4.8 Fluoreszenzspektren der zur Justage verwendeten Farbstoffe . . . 66

4.9 Schematischer Aufbau des NOPA . . . . . . . . . . . . 68 
4.10 Autokorrelation und Spektrum des NOPA . . . . . . . . . 69

4.11 Schematischer Aufbau des Photon-Echo-Experimentes . . . . . . . 71

4.12 Typisches Meßsignal im 3PEPS-Experiment . . . . . . . . . . 75

5.1 Stationäre Spektren für Laurdan in $n$-Hexan, Ethanol und Methanol ............................... 81

5.2 Wärmekapazitätsprofile für DMPC-Vesikel . . . . . . . . . . . 84

5.3 Temperaturabhängige UV-Vis-Spektren von DMPC-Vesikeln . . . 86

5.4 Temperaturabhängige Absorptionsspektren von Laurdan in DMPC . . . . . . . . . . . . . . . . . 88

5.5 Temperaturabhängige Fluoreszenzspektren von Laurdan in DMPC . . . . . . . . . . . . . . . . . . . 89

5.6 Stationäre Spektren für Laurdan in DMPC . . . . . . . . . . . . . 90

5.7 Temperaturabhängige Anisotropiefunktionen für Laurdan in DMPC . . . . . . . . . . . . . . . . . . 93

5.8 Temperaturabhängige Konuswinkel und limitierende Anisotropien für Laurdan in DMPC . . . . . . . . . . . . . . . . 96

5.9 Wobbling-Diffusions- und Rotationsdiffusionskonstante für Laur-

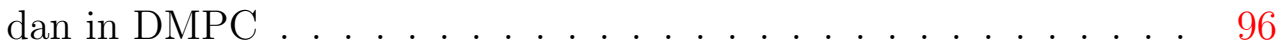

5.10 Zeitaufgelöste Fluoreszenzspektren von Laurdan in DMPC . . . . 98

5.11 Anpassungen von zeitabhängigen Fluoreszenzspektren . . . . . . . 99

5.12 Temperaturabhängige Fluoreszenzlebensdauer und Arrhenius-Auftragung von Laurdan in DMPC . . . . . . . . . . . . . . . 101

5.13 Zeitlicher Verlauf des Schwerpunktes der Fluoreszenzspektren von Laurdan in DMPC . . . . . . . . . . . . . . . . . . 104

5.14 Zeitlicher Verlauf der Halbwertsbreite der Fluoreszenzspektren von Laurdan in DMPC . . . . . . . . . . . . . . . . . . 105

5.15 Zeitlicher Verlauf der Asymmetrie der Fluoreszenzspektren von Laurdan in DMPC . . . . . . . . . . . . . . . . . . 107

5.16 Zeitlich integrierte Fluoreszenzspektren, stationären Emissionsspektren und Fluoreszenzspektren zu langen Verzögerungszeiten . 110

5.17 Solvatationskorrelationsfunktion von Laurdan in DMPC aus dem TCSPC-Experiment . . . . . . . . . . . . . . 112 
5.18 Anpassungsparameter der mit dem TCSPC-Experiment erhaltenen Solvatationskorrelationsfunktion . . . . . . . . . . . . 114

5.19 Mittlere Solvatationszeit in Abhängigkeit der Temperatur . . . . . 115

5.20 Maximal auflösbarer Stokes-Shift und experimentell beobachteter Anteil im TCSPC-Experiment . . . . . . . . . . . . . . 116

5.21 Typische Kinetiken für Laurdan in DMPC . . . . . . . . . . . . 118

5.22 Solvatationskorrelationsfunktion von Laurdan in DMPC . . . . . . 119

5.23 Anpassungsparameter der mit dem FUC-Experiment erhaltenen Solvatationskorrelationsfunktion . . . . . . . . . . . . . 121

5.24 Vergleich der Temperaturabhängigkeit des Integrals unter der zeitabhängigen Anisotropie und der mittleren Solvatationszeit . . . . 122

5.25 Gegenüberstellung der Anpassungsparameter der Anisotropiefunktion und der Solvatationskorrelationsfunktion . . . . . . . . . . 123

5.26 Vergleich der charakteristischen Relaxationszeiten für reines Wasser mit den in FUC-Experimenten ermittelten Zeitkonstanten . . 127

6.1 Ternäres Phasendiagramm des Systems Isooktan/AOT/Wasser . . 133

6.2 Stationäre Spektren für HIDCI in reinem Wasser und inversen Mizellen . . . . . . . . . . . . . . . . . . 135

6.3 3-Puls Photon-Echos und Autokorrelationen bei verschiedenen Verzögerungszeiten $t_{23}$ für HIDCI in reinem Wasser . . . . . . . . 137

6.4 Peakshift-Funktion von HIDCI in reinem Wasser . . . . . . . . 138

6.5 Peakshift-Funktionen für inverse Mizellen mit $\mu=1,2$ und 4 . . . 143

6.6 Peakshift-Funktionen für inverse Mizellen mit $\mu=8,16$ und 32 . 144

6.7 Anpassungsparameter der im Photon-Echo-Experiment erhaltenen Peakshift-Funktionen für inverse Mizellen und reines Wasser . . . 146

6.8 TG-Messungen für HIDCI in reinem Wasser und inversen Mizellen ...................... . 150

6.9 Ramanspektrum von HIDCI in Ethanol und Leistungsspektrum der TG-Messung von HIDCI in Wasser . . . . . . . . . . . . . . . 151

6.10 Leistungsspektren der TG-Messungen von HIDCI in reinem Wasser und in inversen Mizellen . . . . . . . . . . . . . . . . . . 152 



\section{Tabellenverzeichnis}

3.1 Abklingverhalten der Solvatationskorrelationsfunktion in neutralen, kationischen und anionischen Mizellen bestimmt durch zeitkorreliertes Einzelphotonenzählen . . . . . . . . . . . 26

3.2 Abklingverhalten der Solvatationskorrelationsfunktion im System Wasser/AOT/ $n$-Heptan bestimmt durch zeitkorreliertes Einzel-

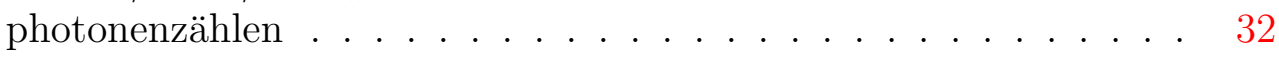

3.3 Abklingverhalten der Solvatationskorrelationsfunktion im System Wasser/AOT/Isooktan bestimmt durch Fluoreszenzaufkonvertie-

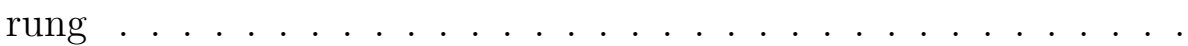

3.4 Mittlere Solvatationszeit in den Lipidmembranen DMPC und DHPC in Abhängigkeit der Temperatur bestimmt durch zeitkorreliertes Einzelphotonenzählen . . . . . . . . . . . . .

3.5 Abklingverhalten der Solvatationskorrelationsfunktion in den Lipidmembranen DMPC und DPPC bestimmt durch zeitkorreliertes Einzelphotonenzählen . . . . . . . . . . . . . . .

4.1 Zur Herstellung der Vesikel verwendete Chemikalien . . . . . . . . 76

4.2 Zur Herstellung der inversen Mizellen verwendete Chemikalien . . 77

5.1 Aufnahmeparameter der stationären Spektren von Laurdan in $n$ Hexan, Methanol und Ethanol . . . . . . . . . . . . . 80

5.2 Absorptions- und Emissionsmaxima von Laurdan in $n$-Hexan, Methanol und Ethanol . . . . . . . . . . . . . . . 83

5.3 Phasenübergangstemperaturen der untersuchten LipidmembranVesikel . . . . . . . . . . . . . . . . . 85

5.4 Anpassungsparameter der Anisotropiefunktion in Abhängigkeit der Temperatur . . . . . . . . . . . . . . 94 
5.5 Auswertung der Anisotropiefunktion nach dem Wobble-in-ConeModell in Abhängigkeit der Temperatur . . . . . . . . . . . . . . 95

5.6 Temperaturabhängige Fluoreszenzlebensdauer und Zeitkonstante des desaktivierenden Kanals . . . . . . . . . . . . . . 100

5.7 Ermittelte Schwerpunkte der Zeitnullpunkts- und Zeitunendlichspektren, sowie resultierender Stokes-Shift . . . . . . . . . . . 111

5.8 Anpassungsparameter der mit dem TCSPC-Experiment erhaltenen Solvatationskorrelationsfunktion . . . . . . . . . . . 113

5.9 Anpassungsparameter der mit dem FUC-Experiment erhaltenen Solvatationskorrelationsfunktion . . . . . . . . . . . . 120

6.1 Zusammensetzung der untersuchten Mikroemulsionen . . . . . . . 132

6.2 Parameter inverser Mizellen in Abhängigkeit von $\mu$. . . . . . . . 134

6.3 Anpassungsparameter für die Peakshift-Funktion in reinem Wasser . . . . . . . . . . . . . . . . . . . . 139

6.4 Anpassungsparameter für die Peakshift-Funktionen in inversen Mizellen und reinem Wasser . . . . . . . . . . . . . . . . . . . 145 


\section{Lebenslauf}

Am 4. April 1976 wurde ich, Marco Thomas Seidel, als Sohn von Gerda Seidel, geb. Seidler und Gerhard Seidel in Kassel geboren.

Nach dem Besuch der Grundschule Helsa von 1982 bis 1986 wechselte ich im September 1986 auf die Valentin-Traudt-Gesamtschule in Großalmerode. Von 1992 bis 1995 besuchte ich das Oberstufengymnasium Herderschule in Kassel, an dem ich am 21. Juni 1995 das Zeugnis der allgemeinen Hochschulreife erhielt.

Im Oktober 1995 immatrikulierte ich mich an der Universität Gesamthochschule Kassel im Diplomstudiengang Chemie und legte dort am 6. Oktober 1997 die Diplom-Vorprüfung ab.

Zum Oktober 1997 wechselte ich an die Georg-August-Universität Göttingen, um den Diplomstudiengang Chemie fortzuführen. Im Juli 1999 begann ich unter der Leitung von Herrn Prof. Dr. Troe am Institut für physikalische Chemie meine Diplomarbeit mit dem Thema „Experimentelle und theoretische Untersuchungen an schwingungsheißem $\mathrm{CO}_{2}$ in Lösung“, welche ich am 8. Mai 2000 mit der Diplomprüfung abschloß.

Seit Januar 2001 bin ich als wissenschaftlicher Mitarbeiter in der unabhängigen Arbeitsgruppe „Biomolekulare und Chemische Dynamik“ unter der Leitung von Herrn Prof. Dr. Vöhringer am Max-Planck-Institut für biophysikalische Chemie in Göttingen angestellt. Hier beschäftigte ich mich mit dem Aufbau eines Fluoreszenzkonversionsexperimentes und Untersuchungen zur Solvatationsdynamik an biologischen Grenzschichten. Als Ergebnis entstand die vorliegende Dissertation. 
